\title{
Análisis y regulación de fluctuaciones de potencia en parques eólicos
}

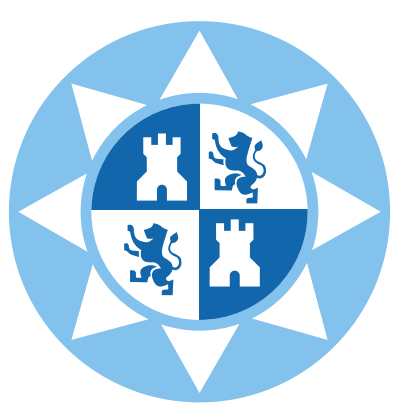

TESIS DOCTORAL

Sergio Martín Martínez

Departamento de Ingeniería Eléctrica

Escuela Técnica Superior de Ingeniería Industrial

Universidad Politécnica de Cartagena

Abril 2013 



\section{Análisis y regulación de fluctuaciones de potencia en parques eólicos}

Autor

Sergio Martín Martínez

Directores

Emilio Goméz Lázaro Antonio Vigueras Rodríguez

Departamento de Ingeniería Eléctrica Escuela Técnica Superior de Ingeniería Industrial Universidad Politécnica de Cartagena

Abril 2013 

No pidas una carga ligera, pide unas espaldas fuertes.

Theodore Roosevelt 

A Marimar

y a mis padres 



\section{Agradecimientos}

Tras varios años de penas y alegrías, de aprender tantas cosas nuevas, y sobre todo después de mucho trabajo, este capítulo de mi vida toca su fin. Y con la ilusión de que todo vaya bien a partir de ahora, afronto una nueva etapa.

Así quiero dar las gracias a mis directores de Tesis, Emilio Gómez Lázaro y Antonio Vigueras Rodríguez que, con su conocimiento y experiencia han sabido guiarme, han depositado en mí una gran confianza y han despejado mi camino de obstáculos a través de proponer nuevas ideas y de saber motivarme.

Por supuesto, también quiero agradecer a los que han sido mis compañeros, a la par que amigos, el gran apoyo que me han dado, haciendo que las interminables horas frente al ordenador hayan sido un poco más llevaderas y hacer nuestro puesto de trabajo un lugar más ameno en el que desarrollar y compartir experiencias laborales y personales.

Un agradecimiento con mayúsculas, debo dárselo a mi familia, por haberme comprendido en los momentos difíciles y haberme soportado en esos momentos en que uno casi se vuelve insoportable. Y muy especialmente a mis padres, por haberme dado tanto cariño, y por haber dedicado tanto a la educación y formación de su hijo.

Y, finalmente, pero no por ello menos importante, a mi mujer, Maria del Mar, por ser mi principal apoyo en todo lo que hago y compartir todo lo bueno y lo malo conmigo.

Enhorabuena, porque este logro es tan vuestro como mío. 



\section{Resumen}

El gran aumento del uso de la energía eólica para la producción de energía eléctrica hace esencial una investigación profunda de esta tecnología y de cómo interactúa con la red a la que se conecta. Las características intrínsecas de este tipo de energía establecen que sea altamente fluctuante y poco gestionable. Esto dificulta el mantenimiento del sistema en rangos óptimos para su funcionamiento. Además los problemas se acentúan en sistemas aislados o con conexiones limitadas. Por ello, para entender y optimizar la producción de las instalaciones eólicas conectadas a red así como del resto de plantas de generación eléctrica es necesario tener un amplio conocimiento de la naturaleza de estas fluctuaciones. Así mismo, se debe tener en cuenta los efectos que estas fluctuaciones pueden ocasionar en el sistema según los diversos rangos de tiempo en los que ocurren. Con el fin de minimizar estos efectos es necesario disponer de herramientas de regulación que reduzcan las fluctuaciones con el mínimo coste energético. De esta forma, se podrían mantener unas condiciones de operación adecuadas en el sistema eléctrico, con una proporción eólica significativamente superior a la actual.

En la presente tesis, se han estudiado los parámetros y rangos que determinan las fluctuaciones de potencia activa de sistemas eólicos conectados a red. Con el objetivo de establecer una guía para un estudio de la variabilidad del viento a nivel de parque y de red. Además, se han descrito diferentes modelos de viento para parque, así como para agregaciones de parques válidos para un estudio analítico de las oscilaciones de potencia. Del mismo modo se han analizado los efectos derivados de las fluctuaciones de potencia, haciendo hincapié en el rango que abarca desde minutos hasta varias horas equivalente a la regulación secundaria y terciaria. Por último, se ha estudiado la implantación de diferentes métodos de regulación para reducir los efectos perjudiciales de la variabilidad en la producción de energía eólica dentro del rango de tiempo mencionado. Dichos métodos se basan en herramientas disponibles para el control de la potencia activa de salida de un aerogenerador que pueden ser utilizadas.

Para ello, se han realizado los consecuentes análisis y validaciones basadas en datos reales de las medidas de potencia y de viento registradas en numerosos parques eólicos distribuidos por la geografía española. 



\section{Abstract}

A wide research of wind power and its interaction with other technologies in the power system is essential because of the large increase in the use of wind energy for electricity production. The intrinsic characteristics of wind power lead to high power fluctuations and some uncertainty in the production. These issues increase the difficulty to keep the system in optimal ranges for operation. Besides, the problems created by these fluctuations are accented in isolated or power systems with limited interconnections. Therefore, to understand and optimize the production of grid-connected wind farms as well as from other power plants is necessary to have extensive knowledge of the nature of these fluctuations. The effect of these phenomena on the power system have to be analyzed according to the different time scales in which they occur. In order to minimize these effects is necessary to have regulatory tools which can reduce fluctuations with minimum energy cost. Thus, it could maintain proper operating conditions in power system, achieving higher proportions of wind power.

In this thesis, a study the parameters and ranges that determine active power fluctuations of grid connected wind systems is presented. The goal is to establish a guide for the analysis of the variability in different aggregations of wind farms. In addition, several developed wind models have been studied for an analytical study of the oscillations of the wind power production. Furthermore, the effects of power fluctuations have been analyzed, emphasizing the ranges of time from minutes to several hours equivalent to secondary and tertiary regulation. Finally, the implementation of different control methods to reduce or eliminate the harmful effects of variability in wind energy production has been studied in the time ranges discussed above. Furthermore, the available tools to control the active power output of a wind turbine that can be used to achieve control methods exposed have been analyzed.

Fluctuations parameters, their effects and improvements due to the implementation of control methods are studied using real active power, wind speed and wind direction measurements recorded at different wind farms over Spain and over several aggregations. These data also have been used to establish the influence of the terrain, to perform a comprehensive analysis of the fluctuations and the corresponding validation of the methods used. 



\section{Índice}

$\begin{array}{ll}\text { Agradecimientos } & \text { IX }\end{array}$

Resumen $\quad$ XI

$\begin{array}{lll}\text { Abstract } & \text { XIII }\end{array}$

1. Introducción y antecedentes 1

1.1. Situación de actual de la energía eólica . . . . . . . . . . . 1

1.2. Estudios de integración de la energía eólica . . . . . . . . 5

1.3. Objetivos de la tesis doctoral . . . . . . . . . . . . . 6

1.4. Estructura de la tesis doctoral . . . . . . . . . . . . 7

2. Variabilidad y predictibilidad de la energía eólica y sus efectos sobre la red eléctrica $\quad 11$

2.1. Introducción . . . . . . . . . . . . . . . . 11

2.2. Análisis de fluctuaciones de la velocidad del viento . . . . . . 13

2.2.1. Análisis espectral de la velocidad del viento . . . . . . 15

2.2.2. Análisis temporal y probabilístico de la velocidad del viento . . . . . . . . . . . . . . 25

2.3. Análisis de fluctuaciones de potencia eólica . . . . . . . . 32

2.3.1. Medida y caracterización de las fluctuaciones de potencia eólica . . . . . . . . . . . . . 35

2.3.2. Fluctuaciones según rangos de potencia . . . . . . . 36

2.3.3. Efecto de suavizado (Smoothing effect) . . . . . . . . . 39

2.3.4. Eventos extremos. Causas y clasificación . . . . . . . 43

2.4. Predicción de la energía eólica . . . . . . . . . . . . 45

2.4.1. Errores de predicción . . . . . . . . . . . . . . 46

2.4.2. Horizontes de predicción . . . . . . . . . . . . . 48

2.4.3. Modelos de predicción del viento . . . . . . . . . . . 49

2.4.4. Modelos de predicción de potencia eólica . . . . . . . . 53

2.4.5. Ejemplos de sistemas de predicción de potencia eólica 56 
2.5. Efectos de la variabilidad de la energía eólica en el sistema eléctrico . . . . . . . . . . . . . . 63

2.5.1. Efectos sobre las reservas del sistema . . . . . . . . 65

2.5.2. Efectos sobre la generación térmica e hidráulica. . . . 70

2.5.3. Recortes de generación eólica . . . . . . . . . . . . . 74

2.6. Conclusiones . . . . . . . . . . . . . . . . . 77

3. Datos utilizados $\quad 79$

3.1. Descripción de los datos disponibles . . . . . . . . . . 79

3.2. Tratamiento de datos y filtrado . . . . . . . . . . . . . 82

3.3. Parámetros de los parques eólicos estudiados . . . . . . . . . . 82

4. Características y efectos de la variabilidad de la potencia eólica en España

4.1. Introducción . . . . . . . . . . . . . . . . . . . . . . . . . 89

4.2. Características regionales de la variabilidad de la potencia eólica en España . . . . . . . . . . . . . . . . . . . . 96

4.3. Características anuales de la variabilidad de la potencia eólica en España . . . . . . . . . . . . . . . . . . . . . 998

4.4. Rampas de potencia eólica extremas y sus causas en España . 98

4.5. Predicción de la energía eólica en el sistema eléctrico español 101

4.6. Evolución de las reservas en el sistema eléctrico español . . . 105

4.7. Ejemplos de eventos extremos . . . . . . . . . . . . . 105

4.7.1. Fenómenos meteorológicos. Tormentas, ciclones y calmas 106

4.7.2. Recortes por saturación de la generación . . . . . . . 110

4.7.3. Perturbaciones de la red. Huecos de tensión y faltas . 112

4.8. Conclusiones . . . . . . . . . . . . . . . . . . 113

5. Métodos de regulación de la potencia activa eólica 119

5.1. Introducción . . . . . . . . . . . . . . . . . 120

5.2. Métodos de Limitación de Rampa Positiva . . . . . . . . . . . 127

5.2.1. PRL aplicado a parques de forma individual . . . . . . 130

5.2.2. PRL aplicado a la producción agregada global . . . . . 131

5.2.3. PRL aplicado según rangos de potencia . . . . . . . . 133

5.2.4. PRL aplicado a los parques con mayores fluctuaciones 135

5.2.5. PRL aplicado a la producción total de España . . . . . 137

5.3. Regulación con constante Delta y NRL . . . . . . . . . . . 138

5.4. Control de tormenta (Storm Control) . . . . . . . . . . . 139

5.5. Conclusiones . . . . . . . . . . . . . . . . . . 146

6. Conclusiones, aportaciones y trabajos futuros 147

6.1. Conclusiones . . . . . . . . . . . . . . . . 147 
6.2. Aportaciones . . . . . . . . . . . . . . . . . . . 148

6.3. Trabajos futuros . . . . . . . . . . . . . 150

A. Estimación de la frecuencia de red mediante un DPLL trifási-

co y media no lineal 153

A.1. Introducción . . . . . . . . . . . . . . . . 153

A.2. Estructuras DPPL . . . . . . . . . . . . 155

A.2.1. Estimación del Vector Espacial de la Tensión . . . . 156

A.2.2. Estimación del error de fase . . . . . . . . . . . . 159

A.3. DPLL trifásico: Solución propuesta . . . . . . . . . . . . 160

A.3.1. Estructura del DPLL trifásico . . . . . . . . . . . . . . 160

A.3.2. Media no lineal ponderada . . . . . . . . . . . 160

A.3.3. Inicialización y comparación del ángulo de fase . . . 162

A.3.4. Ajuste de PI . . . . . . . . . . . . . . . 162

A.4. Validación de la metodología no lineal propuesta . . . . . . 166

A.5. Resultados experimentales . . . . . . . . . . . . 166

A.5.1. Estimación de frecuencia en hueco de tensión trifásico 170

A.5.2. Estimación de frecuencia en hueco de tensión bifásico . 170

A.5.3. Estimación de frecuencia en hueco de tensión monofásico 170

A.6. Conclusiones . . . . . . . . . . . . . . . . . 172

$\begin{array}{ll}\text { Bibliografía } & 175\end{array}$ 



\section{Índice de figuras}

2.1. Espectro de Van der Hoven . . . . . . . . . . . . . . . 12

2.2. PSD de la velocidad de viento en los aerogeneradores números 2 y 6 del parque eólico WF18 . . . . . . . . . . . 18

2.3. Velocidad de viento y variación de la misma para diversos periodos de medias consideradas en la localización del parque

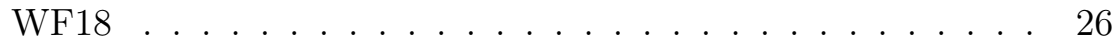

2.4. ACF de la velocidad de viento para tres localizaciones distintas 29

2.5. Función de distribución de probabilidad real y ajustada a una distribución Weibull para los 3 emplazamientos señalados . . 30

2.6. Función de distribución acumulada real y ajustada a una distribución Weibull para 3 emplazamientos señalados . . . . . . 31

2.7. Curva de potencia de un aerogenerador de velocidad variable 32

2.8. Comparación entre la ACF de la velocidad de viento y de la potencia de salida de un aerogenerador . . . . . . . . . . 34

2.9. Función de distribución de probabilidad real de la potencia generada por 3 aerogeneradores en emplazamientos diferentes

2.10. Distribución acumulada de rampas reales para el parque eólico WF1 ...................... 36

2.11. Comparación entre una distribución acumulada de rampas reales y un ajuste mediante una distribución normal a partir de su desviación estándar . . . . . . . . . . . . . 37

2.12. Tipos de curva de potencia según el tipo de sistema de control y accionamiento del ángulo de incidencia . . . . . . . . . . 38

2.13. $99 \%$ rampa negativa según rango de potencia para parques $P<30 M W \ldots \ldots \ldots \ldots \ldots$

2.14. $99 \%$ rampa negativa según rango de potencia para parques $P>30 M W \ldots \ldots \ldots \ldots$. . . . . . . . 40

2.15. $99 \%$ rampa negativa según rango de potencia para producción agregada . . . . . . . . . . . . . . . . 4 41

2.16. Comparación entre la producción de distintas agregaciones eólicas . . . . . . . . . . . . . . . . . . . . 4 42 
2.17. Frecuencia de los eventos según su tamaño mínimo $s_{\text {min }}$ y su duración máxima $d_{\max }$ para el parque WF10 . . . . . . . . 44

2.18. Tipos de error de predicción de energía eólica . . . . . . . . . 47

2.19. Ilustración esquemática de las capas horizontales en la troposfera, que comprende la parte inferior de la atmósfera, importante para la energía eólica. Focken y Lange (2006) . . . . . . 50

2.20. Órdenes de magnitud típicos temporales y espaciales de los fenómenos atmosféricos. Focken y Lange (2006). . . . . . . . . 52

2.21. Esquema básico de Previento, sistema basado en una aproximación física. Focken y Lange (2006) . . . . . . . . . . . 58

2.22. Algoritmos de predicción de Sipreólico. Gonzalez et al. (2004). 59

2.23. Diagrama de funcionamiento de Sipreólico. Gonzalez et al. (2004) . . . . . . . . . . . . . . . . . 5 59

2.24. Esquema del módulo de predicción de Sipreólico. González et al. (2008). . . . . . . . . . . . . . . . 6 61

2.25. Producción de potencia eólica real para las 48 horas anteriores y predicción para las próximas 48 horas para la España peninsular en su conjunto. Gonzalez et al. (2004) . . . . . . . 62

2.26. Impactos de la energía eólica sobre el sistema eléctrico. Holtti-

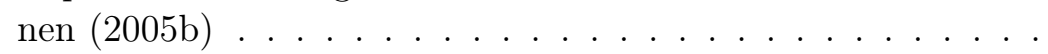

2.27. Curvas de duración de las variaciones de demanda y demanda de red para el año 2009 usando medias horarias. . . . . . . . . .

2.28. Variación de la eficiencia de las centrales térmicas con la carga Gøransson y Johnsson (2010) _ . . . . . . . . . . . 73

3.1. Parques eólicos españoles y agregaciones consideradas. . . . . 81

3.2. Esquema del sistema de registro utilizado para la adquisición del quinto grupo de datos. Martín-Martínez et al. (2012) . . .

4.1. Evolución de la potencia eólica instalada con continuidad de suministro tras hueco de tensión y número de pérdidas de potencia mayores o iguales a $100 \mathrm{MW}$ por huecos de tensión en la red eléctrica española. . . . . . . . . . . . . . . . . 94

4.2. Curvas de duración de parques y agregaciones consideradas. . 97

4.3. Valores de desviación estándar para parques y agregaciones consideradas. . . . . . . . . . . . . . . . . . . 99

4.4. Valores de PNRR99 para parques y agregaciones consideradas. 100

4.5. Desviación estándar de la potencia eólica en el sistema eléctrico español según meses del año . . . . . . . . . . . . . . 101

4.6. Desviación estándar de las rampas de la potencia eólica y PNRR-99 en el sistema eléctrico español según meses del año 
4.7. Parámetros medios de la desviación estándar de las rampas de la potencia eólica y PNRR-99 en el sistema eléctrico español según meses del año . . . . . . . . . . . . . . . . . 103

4.8. Densidad de probabilidad del error de predicción eólica en función de la potencia real entre 2007 y 2011 . . . . . . . . 103

4.9. Ajuste de la media aritmética y la desviación estándar del error de predicción eólica en función de la potencia real a curvas polinómicas de primer, segundo, tercer y cuarto orden

4.10. Ajuste de la desviación estándar del error de predicción eólica por rangos de horas en función de la potencia real a curvas polinómicas de primer, segundo, tercer y cuarto orden . . . . 105

4.11. Comparación de las reservas de regulación desde 2007 a 2011. 106

4.12. Producción eólica, predicción eólica y generación eólica programada durante la tormenta Klaus . . . . . . . . . . . . 107

4.13. Producción eólica del parque WF13 de 49.5 MW durante la tormenta Klaus . . . . . . . . . . . . . . . . . . . . 108

4.14. Producción eólica y predicción eólica con varios horizontes de predicción para el periodo de calma del 4 de febrero de 2007 . 109

4.15. Producción eólica durante la sobrerespuesta del 1 de enero de $2010 \ldots \ldots \ldots \ldots 111$

4.16. Producción eólica y velocidad de viento en el parque WF13 de 49.5 MW durante la sobrerespuesta del 1 de enero de 2010112

4.17. Generación eólica y desvio de la conexión UCTE durante el hueco del 30 de marzo de $2009 . \ldots$. . . . . . . . . . . 114

4.18. Generación eólica y desvío de la conexión UCTE durante el hueco del 7 de noviembre de 2009. . . . . . . . . . . . . 115

4.19. Generación eólica durante los huecos de los días 19 y 20 de marzo de $2007 \ldots \ldots$. . . . . . . . . . . . . 116

4.20. Parques eólicos españoles y agregaciones consideradas. . . . . 117

5.1. Regulación mediante constante absoluta . . . . . . . . . . 120

5.2. Regulación mediante constante Delta de producción . . . . . 121

5.3. Regulación de balance sin cancelación automática . . . . . . . 122

5.4. Regulación de balance con cancelación automática . . . . . . 122

5.5. Regulación de parada . . . . . . . . . . . . . . . . . . 123

5.6. Regulación con constante de gradiente de potencia . . . . . . 123

5.7. Regulación para protección del sistema . . . . . . . . . . . . . 124

5.8. Tipos de control de tormenta. Cutululis et al. (2010). . . . . . 126

5.9. Esquema de aplicación de la regulación PRL . . . . . . . . . . 128

5.10. Ejemplo de pérdidas resultantes de la aplicación de la regulación PRL . . . . . . . . . . . . . . . . . . . . 129

5.11. Esquema de aplicación de la regulación PRL individual . . . . 131 
5.12. Comparativa de tipos de regulación y producción disponible(I) 132

5.13. Reducción de $99 \%$ de rampa negativa y pérdidas para regulación PRL individual y global . . . . . . . . . . . . . 133

5.14. Reducción de la desviación estándar y pérdidas para regulación PRL individual y global . . . . . . . . . . . . 134

5.15. Reducción de rampas negativas PNRR usando PRL individual y global con unas pérdidas de energía del 1\% . . . . . . . . 134

5.16. Comparativa de tipos de regulación y producción disponible (II) . . . . . . . . . . . . . . . . . 135

5.17. Reducción de $99 \%$ de rampa negativa y pérdidas para regulación PRL según rangos . . . . . . . . . . . . . . 136

5.18. Reducción de $99 \%$ de rampa negativa con PRL aplicado a parques con mayores fluctuaciones . . . . . . . . . 137

5.19. Reducción de $99 \%$ de rampa negativa con PRL aplicado a la producción total de España . . . . . . . . . . . . . . . 139

5.20. Reducción de desviación estándar con PRL aplicado a la producción total de España . . . . . . . . . . . . . . . . . 140

5.21. Pérdidas que resultan de la aplicación de la regulación de constante Delta . . . . . . . . . . . . . . . . . . . 140

5.22. Reducción de PNRR-99 y pérdidas para regulación Delta individual y global . . . . . . . . . . . . . . . . . . 141

5.23. Reducción de la desviación estándar y pérdidas para regulación Delta individual y global . . . . . . . . . . . . . . 142

5.24. Esquema de la aplicación de una curva de potencia con control de tormenta . . . . . . . . . . . . . . . . 143

5.25. Comparación de valores reales de potencia con valores obtenidos al aplicar una curva con control de tormenta sobre un aerogenerador del parque WF13 . . . . . . . . . . . . . . . . 144

5.26. Reducción de los valores $\mathrm{PRR}$ al aplicar una curva con control de tormenta sobre el parque WF13 . . . . . . . . . . 145

A.1. Estructura PLL. Implementación de las etapas LF y VCO. . . 156

A.2. Detector de fase (PD): Esquema general. . . . . . . . . . . . 156

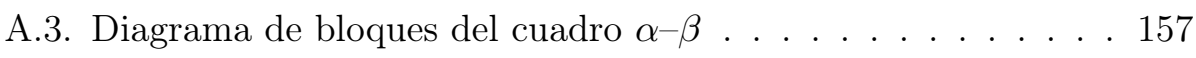

A.4. Solución DPLL trifásico propuesta. Esquema general . . . . . 161

A.5. Cambios en la respuesta de la frecuencia con los pasos simulados para el método MeTa-ArcTg. . . . . . . . . . . . . . 163

A.6. Hueco de tensión simulado con saltos de fase y amplitud. . . . 164

A.7. Comparación de las estimaciones de frecuencia para un hueco de tensión simulado: Topología DPLL trifásico (línea azul) y media ponderada no lineal (línea roja) . . . . . . . . . 165 
A.8. Ejemplos reales de perturbaciones (equilibradas y desequilibradas ) . . . . . . . . . . . . . . . 167

A.9. Ejemplos reales de perturbaciones (equilibradas y desequilibradas). Amplitud estimada . . . . . . . . . . . . . . 168

A.10.Comparación de la estimación de frecuencia para el hueco de tensión trifásico real: Topología DPLL trifásico (línea azul) y media ponderada no lineal (línea roja) . . . . . . . . . . . 169

A.11.Comparación de la estimación de frecuencia para el hueco de tensión bifásico real: Topología DPLL trifásico (línea azul) y media ponderada no lineal (línea roja) . . . . . . . . . . 171

A.12.Comparación de la estimación de frecuencia para el hueco de tensión monofásico real: Topología DPLL trifásico (línea azul) y media ponderada no lineal (línea roja) . . . . . . . . . 173 



\section{Índice de Tablas}

1.1. Capacidad de respuesta y regulación por tecnologías . . . . 3

2.1. Datos estadísticos básicos de la velocidad de viento en el parque WF18 para distintos intervalos de medias temporales durante el mes de Marzo de 2012 . . . . . . . . . . . . . . 27

2.2. Datos estadísticos básicos de las variaciones de la velocidad de viento en el parque WF18 para distintos intervalos de medias temporales durante el mes de Marzo de $2012 \ldots$. . . . . . . 28

2.3. Parámetros de las distribuciones Weibull ajustadas en la figura $2.5 \ldots \ldots \ldots \ldots \ldots \ldots \ldots$

2.4. Operación de los sistemas NWP en Europa . . . . . . . . 51

2.5. Métodos usados para modelos de predicción de velocidad de viento y de potencia eólica. Monteiro et al. (2009) . . . . . . . 54

2.6. Tipología de las reservas . . . . . . . . . . . . . . 67

2.7. Tiempos de arranque de grandes centrales . . . . . . . . . 71

2.8. Tiempos máximo de arranque de centrales . . . . . . . . . . 72

2.9. Capacidades y gradientes típicos de diferentes tecnologías de generación RWE (2009) . . . . . . . . . . . . . . 73

3.1. Parques eólicos estudiados en $2007 \ldots$. . . . . . . . 83

3.2. Parques eólicos estudiados en 2008 . . . . . . . . . . . 83

3.3. Parques eólicos estudiados en 2012 . . . . . . . . . . . . 83

3.4. Combinaciones geográficas de parques eólicos contempladas . 85

3.5. Desviación estándar y valor PNRR-99 a partir de medias cada 10 minutos, 30 minutos y 1 hora de los parques de la tabla 3.186

3.6. Desviación estándar y valor PNRR-99 a partir de medias cada 10 minutos, 30 minutos y 1 hora de los parques de la tabla 3.2 y la tabla $3.3 \ldots \ldots$. . . . . . . . . . . . . . . . . . 87

3.7. Desviación estándar y valor PNRR-99 a partir de medias cada 10 minutos, 30 minutos y 1 hora de la generación eólica total del sistema eléctrico español para los años 2007, 2008, 2009, 2010,2011 y $2012 \ldots \ldots \ldots$. . . . . . . . . . . 87 
4.1. Enumeración de las rampas eólicas más extremas acaecidas en el sistema eléctrico español entre 2007 y 2011 ambos inclusive y sus principales causas . . . . . . . . . . . . . 102

4.2. Sobrerespuesta del recorte del 1 de Enero de 2010 . . . . . . . 113

5.1. Regulación PRL aplicada según los rangos de producción. Valores de reducción porcentual de PNRR-99 dependiendo de las perdidas asociadas. . . . . . . . . . . . 136

5.2. Regulación PRL aplicada a los parques con mayores fluctuaciones. Valores de reducción porcentual de PNRR-99 dependiendo de las pérdidas asociadas. . . . . . . . . . . . . . . 138

A.1. Estructuras de detector de fase (PD): Configuraciones propuestas para señales de entrada monofásicas. . . . . . . . 157 


\title{
Capítulo 1
}

\section{Introducción y antecedentes}

\begin{abstract}
RESUMEN:
El presente primer capítulo de la Tesis Doctoral pretende introducir la temática desarrollada, al igual que los objetivos planteados en la misma. Para comenzar esta parte introductoria, se muestra un resumen del contexto actual de la energía eólica y una muestra de los estudios más importantes sobre integración de la energía eólica en diferentes países. Todo esto junto con las razones que han motivado la Tesis. Finalmente, se muestra la estructura que posee el documento.
\end{abstract}

\subsection{Situación de actual de la energía eólica}

La integración de los parques eólicos en el sistema eléctrico presenta diferentes desafíos para los participantes en la planificación y operación del mismo. Estos desafíos provienen de las características naturales de los parques eólicos, los cuales difieren de las centrales convencionales. Los parques eólicos no son gestionables ya que la potencia inyectada en red varía con la velocidad del viento y por lo tanto es predecible pero no programable. Esto conlleva que los operadores del sistema tengan dificultades añadidas para mantener el balance entre demanda y generación.

Los efectos generados por la energía eólica en la red eléctrica se agravan en sistemas aislados. Un ejemplo de este tipo de sistemas es la Península Ibérica. La Península Ibérica posee unas interconexiones con otras redes muy limitadas. La conexión con Francia apenas permite un intercambio comercial de como máximo $1 \mathrm{GW}$ mientras que la unión con la red marroquí no permite superar los $0.9 \mathrm{GW}$ de intercambio. Aunque existen planes en ejecución de una mejora de la interconexión de la red española se debe pensar en el sistema eléctrico ibérico como una isla eléctrica.

Unido a esto, en España existe una fuerte penetración de la energía eólica en el sistema eléctrico. Actualmente, es innegable la importancia que está 
adquiriendo la energía eólica en el desarrollo de nuestra sociedad, convirtiéndose en uno de los pilares fundamentales de aportación de las energías renovables con un total de 22.649 MW de potencia eléctrica equivalente instalada a Enero de 2013 (datos según la Comisión Nacional de la Energía, CNE (2013)), y representando así en torno al $21 \%$ de toda la potencia eléctrica instalada en nuestro país. Por Comunidades Autónomas, Castilla y León lidera la presencia de generación eólica con 5.481 MW, seguida por Castilla-La Mancha con 3.784 MW y Galicia con 3.339 MW. No sólo en cuanto a potencia instalada, sino también en cuanto a energía producida, la energía eólica se sitúa en el nivel más alto de las energías renovables, cubriendo una media superior al $19 \%$ de toda la energía eléctrica demandada en nuestro país durante el año 2012; lo que supone ocupar el tercer lugar en cuanto a cobertura de la demanda. Incluso, en Enero de 2013 se ha dado la situación en la que más del $29 \%$ de toda la generación eléctrica que se estaba produciendo en el país era proporcionada por las instalaciones eólica.

Adicionalmente a la penetración en la red existen ciertos momentos en los que, debido a las características de la potencia eólica se producen ciertos eventos extremos en los que se reduce la seguridad de la red y se reduce la fiabilidad del sistema. De acuerdo con Red Eléctrica de España, el operador del sistema, la producción de energía eólica alcanzó un nuevo pico de potencia instantánea el 6 de Febrero de 2013, llegando a los 17 GW a las 15:50 del mediodía, que representan un $50 \%$ de la demanda de electricidad peninsular en ese momento, REE (www.ree.es); AEE (www.aeeolica.es). Durante los meses de Marzo y Abril de 2013, la alta producción eólica en combinación con la producción hidroeléctrica como resultado de las altas reservas debidas a las lluvias provocó grandes recortes en la producción térmica y en algunos casos, especialmente durante la madrugada y las horas llano, a la desconexión de grandes cantidades de potencia eólica.

Del mismo modo que en la Península Ibérica, el sistema eléctrico irlandés puede ser considerado como una isla energética. La producción eólica en Irlanda alcanzó un nuevo pico en abril de 2010 tal y como indica la Asociación de Energía Eólica de Irlanda (IWEA), IWEA (www.iwea.com). Se alcanzó un nivel de $1123 \mathrm{MW}$ a las 12:15 del 5 de abril, esto representa un $36 \%$ de la demanda. El viento también ha suplido puntualmente el $50 \%$ de la demanda durante cortos periodos de tiempo en los últimos meses. En el país con mayor penetración eólica en la red como es Dinamarca, la energía eólica llega a cubrir un $60 \%$ de la demanda y se espera que, en breve, se puedan alcanzar picos del $80 \%$ o superiores, Holttinen et al. (2010).

Por otra parte, dentro de la generación, el principal objetivo de la energía eólica es desplazar el uso de combustible fósiles. Las centrales convencionales existentes se encargan de mantener el balance y la fiabilidad del sistema, por lo que no es necesario disponer de generación convencional adicional para realizar el "backup" de los parques eólicos. Sin embargo es necesario opti- 
Tabla 1.1: Capacidad de respuesta y regulación por tecnologías

\begin{tabular}{lllll}
\hline Tecnología & Aporte inercial & Reg primaria & Reg. secundaria & Reg. terciaria \\
\hline Hidráulica & SI & SI & SI & SI $^{a}$ \\
Nuclear & SI & SI & Uso no habitual & Uso no habitual \\
Térmica carbón & SI & SI & SI & SI \\
Ciclo simple gas & SI & SI & SI & SI \\
Ciclo combinado & SI & SI & SI & SI \\
Minihidráulica & SI & & Variable por tecnología \\
Rég. especial térmico $^{c}$ & SI & NO & Variable por tecnología \\
Eólica y fotovoltaica $^{c}$ & NO & NO & NO \\
\hline
\end{tabular}

${ }^{a}$ Límite de disponibilidad

${ }^{b}$ Podría emplearse en seguimiento lento de la demanda

${ }^{c}$ Podría emplearse a bajar

mizar el uso de las reservas aportadas para minimizar riesgos de seguridad y fiabilidad en la operación y reducir sus costes derivados.

Las características y el uso actual de la producción eólica permiten su utilización como reserva a largo plazo en determinadas circunstancias. Este tipo de reservas no cubre los requisitos de operación pero indica que no toda la capacidad instalada va a estar disponible cuando sea requerida. Por el contrario, al no poder gestionar la producción eólica no se puede contribuir a las reservas para el seguimiento de la demanda (secundaria/terciaria) ni a la reserva de regulación (primaria). En todo caso podrían aportar regulación lenta a bajar o a subir si funcionaran por debajo de la máxima potencia y la fuente primaria de energía fuera constante en ese período. Hasta la fecha, tal capacidad no ha sido requerida por la normativa.

En resumen, al no poderse gestionar, la energía eólica no aporta regulación al sistema y además añade requisitos de regulación a otros tipos de tecnologías de forma que su funcionamiento en muchos casos esta fuera de los rangos óptimos. En la tabla 1.1 se establecen las características de las distintas tecnologías de generación en cuanto a la regulación del sistema.

En el aspecto económico, la energía eólica tiene preferencia de acceso a mercado sobre la mayoría de las energías convencionales, esto unido a las primas y ventajas ofrecidas a este tipo de tecnología por su origen renovable hace que la potencia aprovechada se maximice. Esto ofrece un inconveniente añadido para que se pueda aprovechar para realizar servicios de regulación en el sistema. Además, esta situación exige una mayor contribución en la regulación de otras tecnologías con el consiguiente coste económico.

Como una posible solución para mejorar estos problemas de regulación. En sistemas con una gran potencia eólica instalada se hace necesario un control de la potencia activa que permita una regulación óptima teniendo en cuenta el resto de tecnologías disponibles. En la actualidad países como 
Dinamarca e Irlanda establecen en sus "grid codes" la necesidad de estos mecanismos de control. La regulación de la potencia eólica entregada usualmente se realiza por cuestiones de seguridad pero tiene un gran potencial para su uso en la optimización de la generación y en la contribución a la regulación del sistema. Es necesario exponer los distintos métodos de regulación disponibles para regular la producción eólica. De ellos se destacarán aquellos que a priori presentan unas características adecuadas para actuar como herramientas en la reducción de las oscilaciones, destacando el alcance de esa reducción y las pérdidas asociadas.

Existen varias herramientas de control para llevar a cabo las regulaciones que se van a proponer en este documento. Estas herramientas se desarrollan a nivel de aerogenerador, de las cuales las principales son descritas en el capítulo 5. El controlador principal de parques gestiona el control de cada uno de los aerogeneradores de un parque dado. Finalmente en la mayoría de los casos existe un controlador global que rige la producción eólica en esa red. En el sistema eléctrico español el control global se realiza en un centro pionero a nivel mundial para la supervisión y control del régimen especial, el CECRE (Centro de Control para el Régimen Especial).

El CECRE permite integrar en el sistema eléctrico la máxima producción de energía de origen renovable, especialmente eólica, en condiciones de seguridad. Su principal función es supervisar y controlar unidades generadoras de régimen especial y articular la integración de su producción según las necesidades eléctricas del sistema Soto y Prieto (2009). Para este propósito debe:

- Supervisar y controlar las centrales generadoras del régimen especial, con especial hincapié en los parques eólicos.

- Actuar como único interlocutor en tiempo real entre el CECOEL (Centro de Control Eléctrico) y los centros de control de generación, de forma telemática.

- Recibir información necesaria de las unidades de producción para operación en tiempo real, y enviarla al CECOEL.

- Contribuir al cumplimiento de los criterios de operación y seguridad del sistema.

- Realizar un control de la producción real, dirigiendo la tecnologías para alcanzar una mayor producción de energía y una mayor potencia instalada.

- Coordinar el mantenimiento de la red de transporte con el mantenimiento de las conexiones y las instalaciones de generación para minimizar el tiempo durante el cual los generadores son afectados. 
- Capturar los programas de generación gestionable y proporcionar las previsiones de la generación no gestionable (en su mayoría generación eólica).

Todas las instalaciones de régimen especial con una potencia instalada mayor de $10 \mathrm{MW}$ deben estar conectadas al CECRE.

El CECRE, a partir de la información recibida en su Sistema de Control de Energía mediante la utilización de programas de análisis de sistemas de potencia y otros desarrollados específicamente (GEMAS-Generación Eólica Máxima Admisible en el Sistema, CECRE), calcula la producción eólica que en cada momento puede integrarse en el sistema eléctrico en función de las características de los generadores y del estado del propio sistema eléctrico. El cálculo se produce con desglose por parque y agregación por nudo de la Red de Transporte y es enviado a los Centros de Control de Generación, quienes, a su vez, la comunican a los generadores para que procedan a la modificación de la consigna de potencia vertida a la red para su envío a los generadores. Esto permite maximizar la producción eólica dentro de los límites de seguridad del sistema.

En otras regiones, los estudios sobre la integración de la energía eólica nos ofrecen una perspectiva de futuro sobre las necesidades de los sistemas eléctricos para soportar los requerimientos impuestos por la instalación de una determinada potencia eólica.

\subsection{Estudios de integración de la energía eólica}

Para analizar la integración de la energía eólica en los sistemas eléctricos se han realizado diversos estudios en los que se analiza su impacto. En los estudios de integración se analizan diferentes impactos en diferentes escalas de tiempo usando modelos y datos acordes con dicha escala.

La división de la agencia internacional de la energía IEA Wind que se encarga de poner en práctica el acuerdo para la cooperación en la investigación, desarrollo, e implantación de Sistemas de Energía eólica, está desarrollando un estudio de integración llamado Tarea 25 (task 25). Esta tarea se ha desarrollado en una primera fase durante los años 2006-2008, cuyos resultados ya se pueden consultar, Holttinen et al. (2010). Se puso en marcha una extensión del estudio en la fase 2009-2011, cuyos resultados no han sido publicados hasta el momento. El objetivo principal de este estudio es el de proporcionar información para facilitar la penetración de la energía eólica en los sistemas eléctricos de la forma más factible desde el punto de vista económico. Esta tarea lleva a cabo este objetivo analizando y desarrollando la metodología para soportar el impacto de la energía eólica en los sistemas eléctricos. También, se establece un foro internacional para el intercambio de conocimiento y experiencias relacionadas con la operación de sistemas 
con grandes cantidades de instalaciones eólicas. Los participantes recopilan y comparten información de la experiencia ganada y de la investigación realizada durante la tarea. Los casos estudiados abarcan diferentes aspectos de la operación y el diseño del sistema eléctrico: reservas, balance y eficiencia de la generación, eficiencia en el uso de la red de transporte y requerimientos para la mejora de la red (cuellos de botella, intercambios internacionales y problemas de estabilidad del sistema). Los costes de la integración se utilizan como parámetro de comparación para sistemas diferentes. Se propondrán técnicas de operación para ayudar a la integración de la energía eólica, como son: los procedimientos de operación y el control de los parque eólicos, el almacenaje, la gestión de la demanda, gestión de las líneas de transporte, ...

El estudio comienza con un estado del arte recopilando conocimientos y resultados. A partir de lo obtenido en el estado del arte y de las experiencias de los participantes se pretende establecer unas directrices en las metodologías de estimación del impacto en la red y unas recomendaciones para la operación de los sistemas que tengan una alta penetración de energía eólica.

Adicionalmente, existen otros estudios de integración que proponen diferentes casos, centrados en países o regiones concretas, en los que se considera de forma hipotética una cierta cantidad de potencia eólica instalada y como esta, afecta al sistema eléctrico. Ejemplos de este tipo de estudios de integración son los realizados en Ontario (2006), Minnesota (2006), Colorado (2006), Irlanda (2009) y el realizado para Holanda de Brand et al. (2010).

\subsection{Objetivos de la tesis doctoral}

Después de haber realizado una revisión de la literatura existente en el tema, se ha constatado la disponibilidad de diferentes métodos de regulación de la potencia eólica. Diversos autores consideran que dos de estos métodos (limitación delta y limitación de rampa positiva) pueden contribuir al suavizado de las fluctuaciones de potencia eólica. Sin embargo, a priori no se conoce el alcance de los mismos en cuanto a dicho suavizado en conjuntos de parques eólicos ni las pérdidas de energía que ocasionan.

El objetivo principal de la presente Tesis Doctoral es la propuesta de métodos de regulación y control que reduzcan la variabilidad de la energía eólica de forma que se mejore la integración de la misma en las redes eléctricas. Dentro de ese objetivo principal se incluye una serie de objetivos, los cuales se presentan a continuación:

- Describir la diversas herramientas y parámetros para el estudio y la evaluación de fluctuaciones de potencia eólica.

- Destacar los principales efectos a medio y largo plazo de la variabilidad eólica en los sistemas eléctricos. 
- Conocer el alcance del efecto de amortiguación de las fluctuaciones por la agregación espacial de parques eólicos, también llamado efecto de suavizado. El estudio se centrará en la evaluación de este efecto en función del número de parques considerados y la potencia instalada y la distancia entre dichos parques.

- Proponer distintos métodos de regulación para la reducción de las fluctuaciones eólicas.

- Evaluar el alcance de la aplicación de dichos métodos de forma continua sobre la producción eólica de diversas agregaciones y la posible contribución que esto puede suponer para las reservas del sistema. De modo similar, se debe evaluar la posible contribución de la energía eólica a las reservas en ciertos casos mediante estos métodos.

- Analizar las condiciones óptimas para el uso de los métodos de forma que se obtenga la mayor reducción de la variabilidad con las mínimas perdidas energéticas, evaluándose distintas configuraciones de estos métodos aplicadas tanto a parques individuales como agregaciones de los mismos y de forma global a la producción agregada.

- Desarrollar una técnica de estimación de la frecuencia de red bajo condiciones de perturbaciones de red de modo que pueda ser usada en combinación con los métodos de control de potencia activa para poder establecer sistemas robustos de control primario para instalaciones eólicas.

Todos los objetivos propuestos requieren el uso de datos reales para realizar un análisis y una validación correcta de los resultados.

\subsection{Estructura de la tesis doctoral}

La presente Tesis Doctoral está dividida en cinco capítulos, incluido éste y un apéndice. Previamente a esta organización se presenta un breve resumen de la temática investigada así como los índices del documento. Finalmente, se ha dispuesto una lista de las referencias bibliográficas indicadas.

En este primer capítulo se ha presentado una introducción general al contexto actual de la energía eólica como preludio al estudio de las fluctuaciones de potencia eólica. Adicionalmente se ha incluido una descripción de diferentes estudios de integración de la energía eólica en los sistemas eléctricos. De esta manera, se ha permitido mostrar las razones que han motivado la presente investigación, así como los objetivos perseguidos.

En el capítulo 2, se comienza presentando un compendio sobre el origen y las características de las fluctuaciones de potencia eólica. Se plantean 
dos tipos de herramientas para el estudio de la variabilidad, las herramientas espectrales y las herramientas estadísticas. Las fluctuaciones de potencia eólica causadas por dichas oscilaciones se atenúan parcialmente al considerar la producción conjunta de varias unidades. Por ejemplo, las oscilaciones más rápidas del viento suelen amortiguarse en el nivel de parque eólico, mientras que las variaciones de menor frecuencia sólo son suavizadas parcialmente al considerar conjuntos de parques eólicos. Dichas fluctuaciones también varían en función del nivel de potencia al que se encuentra el parque o agregación. Posteriormente se analiza la herramienta más utilizada para minimizar los efectos de la variabilidad eólica, el uso de la predicción de potencia eólica. Se indican los errores típicos y los métodos más usados para realizar la predicción, tanto a nivel local como a nivel regional. Adicionalmente, se muestra el funcionamiento y la composición de dos de los modelos de predicción más utilizados, el Previento y el Sipreólico. Una vez expuestas todas esas características de las fluctuaciones y de su predicción se podrá analizar el alcance de los principales efectos derivados de la naturaleza oscilante del viento, destacando los efectos que la energía eólica tiene sobre el resto del sistema en un rango de tiempo a medio-largo plazo.

Los datos usados en el estudio y validación de la propuesta incluida en esta tesis se describen en el capítulo 3. Estos datos consisten en series temporales con datos de potencia y velocidad de viento de diferentes parques y agregaciones dispuestas a lo largo y ancho de la geografía española. A continuación se presentan las propiedades de los diferentes parques eólicos y las agregaciones consideradas de donde se han obtenido los datos. Finalmente se indican los parámetros básicos de variabilidad de los casos propuestos como referencia.

El capítulo 4 incluye un análisis exhaustivo de las características de la fluctuaciones eólicas y sus efectos para el caso particular del sistema eléctrico español. Se analizan las características regionales y anuales de las fluctuaciones y el efecto de las mismas sobre las reservas y la demanda de red, resaltando su evolución a través de los últimos años. Finalmente se describen varios ejemplos de eventos extremos ocurridos en la red eléctrica española relacionados con la energía eólica y sus fluctuaciones. Estos eventos son clasificados en función de su origen.

En el capítulo 5, se describen los métodos de regulación planteados por diferentes "Grid codes" para el control de la potencia activa de parques eólicos. Seguidamente se seleccionan dos de ellos, el método PRL y el control Delta como candidatos para su utilización en la reducción de la variabilidad de la potencia eólica. Una vez seleccionados, el método PRL es validado utilizando varias configuraciones para conseguir la mayor reducción proporcional de las fluctuaciones con una menor perdida energética. Se comentan los resultados obtenidos y los casos para los que funcionan mejor cada uno de los métodos. Finalmente, de forma análoga al PRL, se realizan los pasos 
anteriores para el control Delta con uso del NRL.

Para finalizar, el capítulo 6 contiene las conclusiones derivadas de la presente Tesis Doctoral. Además, recoge las aportaciones novedosas que han surgido objeto de la investigación y plantea diversas alternativas por las que dar continuidad al trabajo desarrollado.

Como único anexo se ha incluido en el apéndice A un desarrollo y la validación de un método DPLL monofásico con media no lineal ponderada para la estimación de frecuencia de red durante perturbaciones equilibradas y desequilibradas. Este método ha sido estudiado tanto con datos simulados para exponer cláramente su funcionamiento, como con datos reales medidos en un parque eólico para comprobar su validez. 



\title{
Capítulo 2
}

\section{Variabilidad y predictibilidad de la energía eólica y sus efectos sobre la red eléctrica}

\author{
No hay viento favorable para el que no \\ sabe donde va. \\ Lucio Anneo Séneca
}

\begin{abstract}
Resumen: En este capítulo se definen y se describen las principales características de las fluctuaciones de la velocidad de viento y de la potencia activa de salida resultante en los aerogeneradores, los parques eólicos y las agrupaciones o agregaciones de los mismos. Como primer paso se examinan las estadísticas de las series temporales de la velocidad del viento y se explican las variaciones de la velocidad del viento en diferentes escalas de tiempo. A continuación se detalla la conversión de la velocidad de viento a potencia eléctrica y la relación entre las fluctuaciones de potencia y las de velocidad de viento, y las características de dichas fluctuaciones. Seguidamente, se describen los métodos de predicción de la potencia eólica y se resumen dos modelos reales como son el Previento y el Sipreólico. Y por último se resumen los efectos de la energía eólica en la red eléctrica señalándose más en profundidad los principales efectos a medio y largo plazo.
\end{abstract}

\subsection{Introducción}

El viento es una consecuencia de las diferencias de presión atmosférica sobre la superficie de la Tierra. El calentamiento desigual de la superficie de la Tierra y el calor transferido por las corrientes oceánicas son los principales responsables de dichas diferencias de presión. Cerca del suelo, el movimiento 
CAPítulo 2. Variabilidad y predictibilidad de la energía eólica y sus 12 efectos sobre la red eléctrica

del aire se ve afectado también por las diferentes características de la superficie terrestre. El resultado de todos estos factores es que el viento varía todo el tiempo tanto en velocidad como en dirección. Para la producción eólica, la velocidad del viento es de particular interés porque representa el impacto más directo sobre el comportamiento de un aerogenerador, parque eólico o agregación de parques eólicos.

Aunque muchos autores se han referido a la variabilidad o intermitencia de la energía eólica durante décadas, su comportamiento se ha caracterizado a partir de estudios realizados recientemente. Las fluctuaciones del flujo atmosférico son bastante conocidas, siendo el espectro de viento de Van der Hoven mostrado en la figura 2.1 la representación más común de su contenido de energía en el rango meteorológico. De esta forma se muestra la densidad de potencia espectral para cada frecuencia, destacando 3 zonas diferenciadas. La primera zona es denominada "pico macrometeorológico", está localizada en la zona de altas frecuencias y consiste en un pico de energía centrado en una frecuencia aproximada de 0.01 ciclos/hora, la segunda se denomina "hueco espectral", se localiza en frecuencias medias y consiste en una extensión de baja energía desde una frecuencia de 0.2 ciclos/hora hasta 20 ciclos/hora, y finalmente la última zona es la llamada "pico micrometeorológico" la cual se extiende en frecuencias altas de forma que el pico está centrado en 60 ciclos/hora.

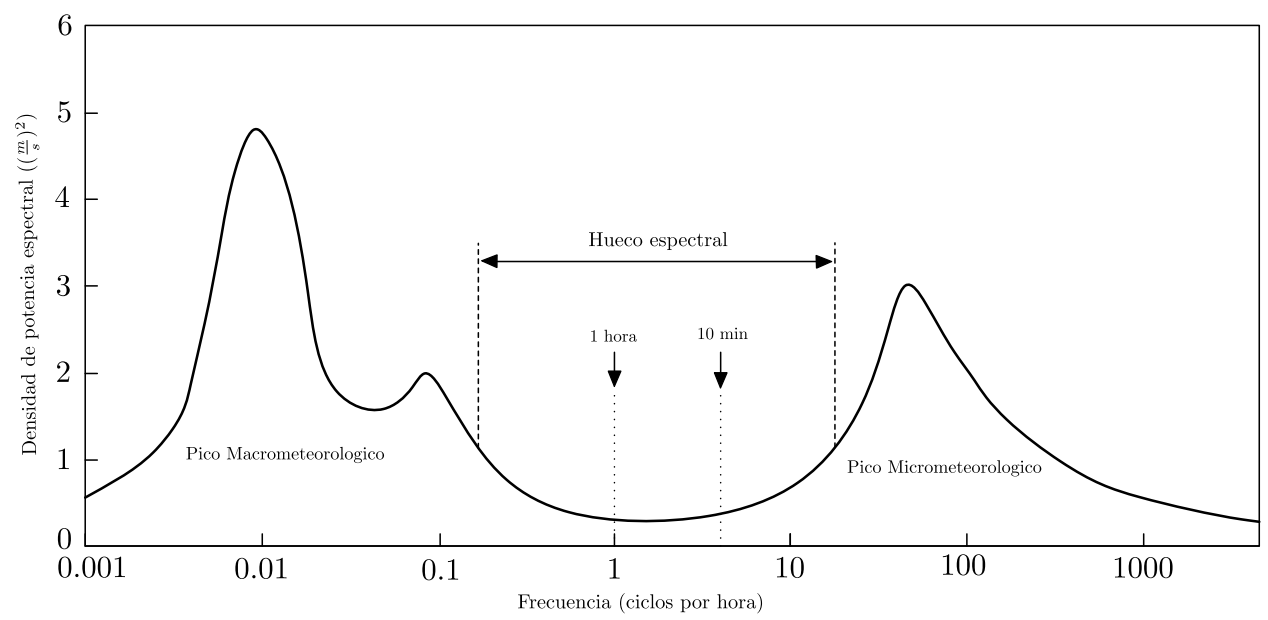

Figura 2.1: Espectro de Van der Hoven

Las fluctuaciones de potencia eólica son mas difíciles de caracterizar y es actualmente cuando se empieza a conocer su naturaleza. La razón de esa situación parte parcialmente del hecho de que, aunque la medición del recurso eólico se extiende a niveles locales, regionales, de un país o, incluso, a escalas de continentes y, por lo general, el recurso eólico se registra con sistemas de adquisición de datos simultáneos y sincronizados, la información no es 
directa y fácil de transferir a la energía eólica por varias razones. En primer lugar, los aerogeneradores son equipos con un comportamiento no lineal, es decir, la relación entre potencia y velocidad de viento $P=f(v)$ es una función no-lineal, y muchas veces no biyectiva. En segundo lugar, la velocidad de viento no posee una distribución de densidad de probabilidad Gaussiana; por el contrario muestra una fuerte asimetría con respecto a su media, siendo sus fluctuaciones normalmente representadas mediante una función de distribución Weibull. En tercer lugar, el hecho de que el flujo de viento de la atmósfera es tridimensional, y las variables meteorológicas no son estacionarias ni ergódicas, no contribuye tampoco a una relación directa, como indican Davenport (1961); Kaimal et al. (1972). En cuarto lugar, la forma y la distribución de las agrupaciones de aerogeneradores influye fuertemente en la potencia generada y por lo tanto en la variabilidad de la misma. Todo lo señalado anteriormente se suma al hecho de que la red a la que se incorpora un aerogenerador o parque eólico tiene también un impacto (generalmente a través de la potencia de cortocircuito en el punto de conexión y los transformadores de interconexión) en la fase y la amplitud de las fluctuaciones generadas por la unidad de potencia. La situación descrita ha dificultado que la mayoría de los autores actuales obtengan las características típicas de las fluctuaciones generadas por las plantas de energía eólica, especialmente en agregaciones con una alta capacidad instalada.

Además, estas características inherentes de los aerogeneradores hacen que exista una diferencia entre las fluctuaciones de potencia eólica y las fluctuaciones de la velocidad de viento. En determinados momentos y bajo determinadas condiciones se producen eventos que generan fluctuaciones de potencia eólica muy superiores a las que cabría esperar asociadas a las fluctuaciones de la velocidad de viento. Estos eventos extremos son muy difíciles de caracterizar en el dominio del tiempo y especialmente en el dominio de la frecuencia, y suelen representar problemas en la calidad y la seguridad del sistema eléctrico.

\subsection{Análisis de fluctuaciones de la velocidad del viento}

La velocidad horizontal de viento, a la cual nos referiremos a partir de ahora, es una variable estocástica que varía temporal y espacialmente, y está acotada inferiormente. Se puede considerar, para facilitar su modelización, que la velocidad del viento está constituida por la suma de cuatro componentes, como presentan Slootweg (2003) y Burton et al. (2001):

$$
v_{v}(t)=v_{v m}+v_{v r}(t)+v_{v r a f}(t)+v_{v t}(t)
$$

siendo: 
CApítulo 2. Variabilidad y predictibilidad de la energía eólica y sus efectos sobre la red eléctrica

- $v_{v m}$ es el valor promedio de la velocidad del viento. El cual corresponde al promedio aritmético de la velocidad del viento registrada durante un cierto período de tiempo considerado. Por lo general, esta información es fácilmente extraíble de una serie de datos de velocidad del viento, mediante un simple tratamiento estadístico. Este valor es de suma importancia en estudios de medida del recurso eólica, sin embargo para el estudios de la variabilidad de la velocidad de viento no es un valor crítico.

- $v_{v r}(t)$ es la componente rampa. Es utilizada para representar un incremento o decremento continuo en la velocidad del viento. La componente de rampa se emplea para la simulación de cambios en la velocidad de viento, cuyo comportamiento puede ser simulado en una forma más o menos lineal (ascenso o descenso). Esta rampa en la velocidad del viento es caracterizada por tres parámetros: la amplitud de la rampa, $A_{r}(\mathrm{~m} / \mathrm{s})$, el tiempo de comienzo o inicio de la rampa $T_{s r}$ en segundos, y el tiempo final de la rampa, $T_{e r}$ en segundos. La formulación matemática de la componente de rampa de la velocidad del viento se describe en las siguientes ecuaciones:

$$
\begin{array}{ccc}
v_{v r}=0 & \text { si } & t<T_{s r} \\
v_{v r}=A_{r} \frac{\left(t-T_{s r}\right)}{\left(T_{e r}-T_{s r}\right)} & \text { si } & T_{s r} \leq t \leq T_{e r} \\
v_{v r}=A_{r} & \text { si } & t>T_{e r}
\end{array}
$$

Desde el punto de vista de la variabilidad, la pendiente $m_{r}$ de la rampa es un parámetro de suma importancia. En la ecuación 2.3 se describe el valor de la pendiente en función de los parámetros de amplitud y duración de la misma.

$$
m_{r}=\frac{A_{r}}{T_{e r}-T_{s r}}
$$

- $v_{v r a f}(t)$ es el componente de ráfaga, representando una ráfaga en el viento. Las ráfagas son fluctuaciones rápidas en la velocidad del viento, con cierta variación entre los picos y la parte estable de la curva, es decir un cambio temporal en la velocidad del viento, tal y como se describe en Slootweg et al. (2003).

La ráfaga está caracterizada por tres parámetros: la amplitud, $A_{\text {raf }}(\mathrm{m} / \mathrm{s})$, el tiempo de comienzo o inicio $T_{\text {sraf }}$ en segundos, y el tiempo final $T_{\text {eraf }}$ en segundos. La formulación matemática de la componente de ráfaga de la velocidad del viento se describe en las siguientes ecuaciones: 


$$
\begin{array}{ccc}
v_{\text {vraf }}=0 & \text { si } & t<T_{\text {sraf }} \\
v_{\text {vraf }}=A_{r}\left[1-\cos \left(2 \pi \frac{\left(t-T_{\text {sraf }}\right)}{\left(T_{\text {eraf }}-T_{\text {sraf }}\right)}\right)\right] & \text { si } & T_{\text {sraf }} \leq t \leq T_{\text {eraf }} \\
v_{\text {vraf }}=0 & \text { si } & t>T_{\text {eraf }}
\end{array}
$$

- $v_{v t}(t)$ es la componente que representa la turbulencia. La turbulencia del viento es una función sumamente compleja, debido a que involucra un comportamiento errático y poco predecible de la velocidad del viento, lo que dificulta la caracterización matemática de esta componente. El comportamiento de la turbulencia depende de varios factores como son: origen de la turbulencia (capa límite atmosférica, rugosidad superficial, etc), intensidad y espectro. Este componente ha sido objeto de numerosos estudios a lo largo de los años, como en Ackermann (2003), y se ha descrito que posee distintos enfoques para su simulación, definidos en función del fenómeno a estudiar y el lugar donde se esté llevando a cabo el estudio. Una forma de realizar la caracterización de la turbulencia es considerando tres aspectos: la intensidad de la turbulencia, el espectro de turbulencia y las escalas de longitud.

Para el análisis de la variabilidad de la velocidad del viento, es necesario tener en cuenta todas las componentes variables. Sin embargo, dependiendo del periodo elegido entre muestras y la longitud de la serie temporal disponible para el estudio tendrán más peso unas componentes que otras. Tradicionalmente, la componente de turbulencia $v_{v t}(t)$ no era considerada para periodos entre muestras significativamente superiores a 10 minutos. Del mismo modo, el componente de ráfaga $v_{\text {vraf }}(t)$ solo es relevante en periodos que van desde unos segundos hasta un minuto, rango este correspondiente al pico micrometeorológico del espectro de Van der Hoven. Por el contrario, la componente rampa $v_{v r}$, al tratarse de la suma de variaciones persistentes, se puede aplicar a cualquier periodo entre muestras de estudio.

$\mathrm{El}$ análisis de las fluctuaciones de viento se puede realizar mediante espectral o de forma estadística. En los siguientes apartados se describen ampliamente estas dos herramientas de análisis.

\subsubsection{Análisis espectral de la velocidad del viento}

Las fluctuaciones en la velocidad de viento se pueden considerar como el resultado de una combinación de velocidades de vientos variables sinusoidales sumadas a la velocidad de viento media constante. Estas variaciones 
CApítulo 2. Variabilidad y predictibilidad de la energía eólica y sus 16 efectos sobre la red eléctrica

sinusoidales poseen distintas frecuencias y amplitudes. El término "Espectro" se utiliza para describir la transformación de la velocidad de viento al dominio de la frecuencia.

El espectro de viento de Van der Hoven muestra una depresión entre las frecuencias de 3 minutos por ciclo y 5 horas por ciclo. Esta depresión se denomina hueco espectral, el cual separa las fluctuaciones rápidas de viento (flujo turbulento) de las fluctuaciones lentas (flujo medio). Esta representación del espectro de viento es la más común pero suele variar dependiendo de la localización, como se indica en Van der Hoven (1957). El hueco espectral ha sido identificado en otras localizaciones por diversos autores, como son Courtney y Troen (1990); Yahaya et al. (2003).

Basándose en el hueco espectral, descrito en la figura 2.1, se puede asumir que habrá poca variabilidad de la velocidad de viento en esas escalas de tiempo, lo que permitía desacoplar las fluctuaciones en ambas escalas. Sin embargo, se ha demostrado que el hueco espectral no siempre está bien definido y bajo ciertas condiciones atmosféricas no existe tal hueco en esas frecuencias, ocurriendo en los estudios de Gjerstad et al. (1995); Heggem et al. (1998). Las fluctuaciones de la velocidad de viento en estas escalas de tiempo no son una característica constante del viento y presentan un patrón meteorológico según la época del año, la hora del día y otras variables.

El procedimiento usado para el análisis espectral de series temporales de viento está basado en el método de Welch (1967). En este método la serie temporal se divide en bloques de la misma longitud y se calculan los estimadores espectrales, conocidos como periodogramas, para cada bloque. Finalmente, se realiza la media de los estimadores de los distintos bloques, obteniendo el periodograma para toda la serie temporal. Se puede usar tanto para el cálculo del espectro de potencia (densidad espectral de potencia) como para el cálculo de la correlación espectral como se propone en Cooley et al. (1970).Este método es apto para series temporales con datos erróneos o inexistentes ya que se pueden omitir aquellos bloques que contengan datos erróneos o no estén completos. El proceso de cálculo de este método se divide en 3 pasos:

- El primer paso consiste en separar la serie temporal de una localización $j$, denominada como $u_{j}$, en diversos bloques de longitud $N$ muestras de forma que se obtenga una serie para cada bloque, donde para el bloque $m$ se denomine $u_{j, m}$. Para reducir las pérdidas causadas por truncar la serie global, cada bloque temporal se multiplica por una función ventana, $\omega(n)$, como por ejemplo la de Hamming.

- En el segundo paso se calcula la transformada discreta de Fourier para cada bloque: 


$$
\operatorname{DFT}_{j, m}(l)=\sum_{n=0}^{N-1} u_{j, m}(n) \omega(n) e^{(-i 2 \pi l n / N)},
$$

siendo $l=0, \ldots,(N-1)$. Este cálculo se puede realizar usando la transformada rápida de Fourier. Los valores de frecuencia correspondientes a los coeficientes de Fourier son:

$$
f(l)= \begin{cases}f_{s} \cdot \frac{l}{N} & 0<l \leq N / 2 \\ f_{s} \cdot \frac{(l-N)}{N} & N / 2<l<N\end{cases}
$$

- El tercer y último paso consiste en establecer los periodogramas de las series completas a partir de la media de todos los bloques.

\subsubsection{Densidad espectral de potencia de la velocidad de viento}

La función espectral que caracteriza la variabilidad en función de la frecuencia se conoce como la función de densidad espectral. Dado que el valor promedio de cualquier función sinusoidal es cero, las amplitudes se caracterizan en términos del promedio de sus valores al cuadrado. Este tipo de análisis se originó en ciertas aplicaciones de la energía eléctrica, donde los cuadrados de la tensión o la corriente son proporcionales a la potencia, o en el caso de la velocidad del viento que es proporcional a la energía cinética fluctuante. El nombre completo de la función que describe la relación entre la frecuencia y amplitudes de las diferentes ondas sinusoidales que componen las fluctuaciones de la velocidad del viento se denominan, "Densidad Espectral de Potencia" (PSD).

Considerando que el viento está compuesto por una parte constante y determinística $\bar{V}$ y por una parte estocástica $v(x, y, z, t)$ como se indica en 2.7 .

$$
V(x, y, z)=\bar{V}(x, y, z)+v(x, y, z, t)
$$

La PSD viene definida por la siguiente expresión:

$$
P S D(f)=F\left(\phi_{v}(t)\right)_{(f)}=\int_{-\infty}^{+\infty} \phi_{v}(\tau) \exp ^{i 2 \pi f \tau} d \tau
$$

donde $f$ es la frecuencia en $H z$ y $\phi_{v}$ es la autocorrelación de $v$ dada por $\phi_{v}=E[v(t) \cdot v(t+\tau)]$, donde $E[x(t)]$ es el valor esperado de $x(t)$.

La densidad espectral de la variación del viento es a menudo utilizada en los análisis dinámicos. Esta es una medida de la contribución de los rangos de frecuencia a la varianza total, utilizada por Bendat y Piersol (2011); Vanmarcke (2010). 
CAPÍtulo 2. Variabilidad y predictibilidad de la energía eólica y sus

$$
\int_{0}^{\infty} P S D(f) d f=\sigma^{2}
$$

donde $P S D(f)$ es la densidad de potencia espectral de la velocidad de viento y $\sigma^{2}$ es la varianza. PSD:

Así, según 2.9, existen dos propiedades particulares importantes de la

- La varianza de las fluctuaciones de la velocidad de viento en un rango de frecuencias se puede obtener integrando la $P S D(f)$ de la velocidad de viento en ese rango.

- La integral de la $P S D(f)$ de la velocidad de viento para todas las frecuencias es igual a la varianza total de la velocidad de viento.

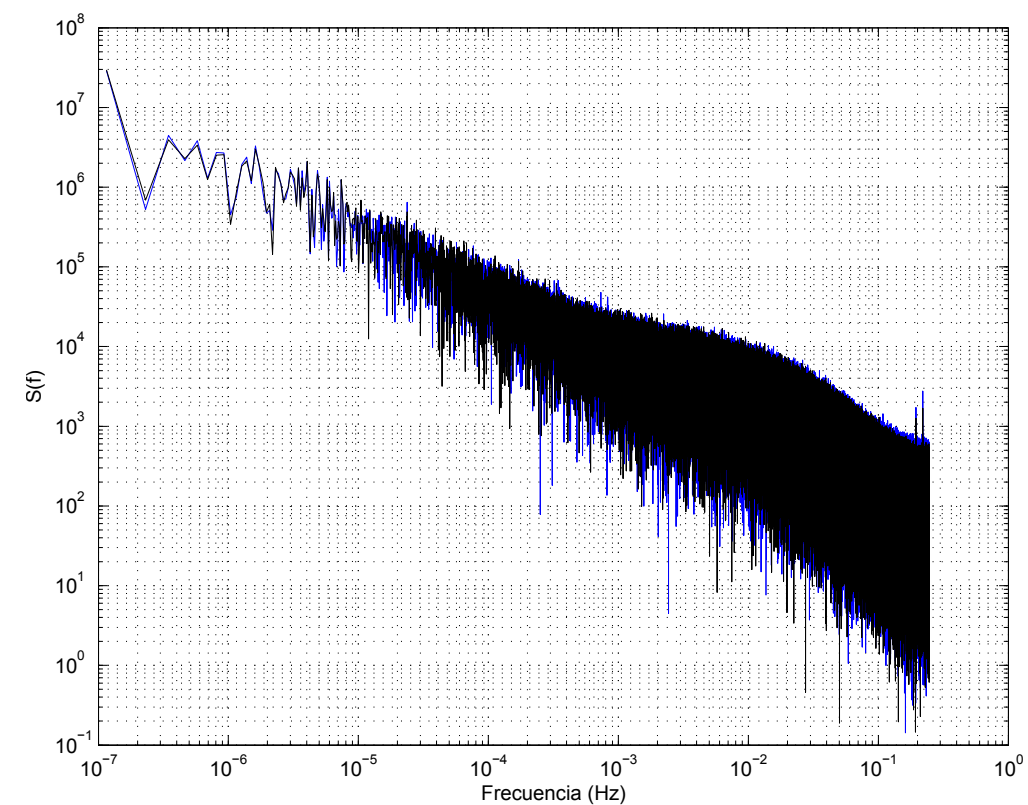

Figura 2.2: PSD de la velocidad de viento en los aerogeneradores números 2 y 6 del parque eólico WF18

En la figura 2.2, se muestran los PSDs de la velocidad de viento medida en dos aerogeneradores del mismo parque eólico (WF18). Por su cercanía, se aprecia una alta correlación. Así las fluctuaciones de baja frecuencia son idénticas, mientras que para altas frecuencias existen pequeñas variaciones.

De acuerdo a lo descrito, las variaciones momentáneas de la velocidad de viento se pueden clasificar de acuerdo a su frecuencia y su extensión espacial. Las fluctuaciones lentas o de baja frecuencia (en el rango de decenas de minutos y horas) se deben principalmente a la dinámica meteorológica y 
tienen una alta correlación entre los vientos registrados en localizaciones o parques eólicos cercanos. Las fluctuaciones rápidas o de alta frecuencia se deben a la turbulencia del viento, al clima local y a la orografía y tienen una menor correlación espacial Nichita et al. (2002); Petru y Thiringer (2002), por lo que los efectos de las ráfagas de viento y la turbulencia se suavizan en la salida del parque eólico. Así, una ráfaga rápida que llega simultáneamente a todas las turbinas dispersas en un área de kilómetros es muy poco común.

\subsubsection{Modelos del espectro de la velocidad del viento}

Las variaciones del viento en la dirección de la corriente del flujo se caracterizan a través de la PSD de la componente longitudinal de la velocidad instantánea. Una serie de funciones de PSD se utilizan como modelos en el campo de la energía eólica cuando los datos de la densidad de potencia espectral de la turbulencia no están disponibles para una cierta localización. Las formas matemáticas de los espectros de velocidad de viento que se utilizan actualmente en los principales estudios, códigos de red, y normativa son el modelo de Von Karman, el de Kaimal modificado, el de Davenport y el de Harris, Tamuraa et al. (2001).

A continuación se detallan los principales modelos utilizados:

El primer modelo usado fue el de Von Karman (1948) El PSD de este modelo viene definido por la siguiente expresión:

$$
P S D_{v k a r m a n}(f)=\frac{4 \cdot\left(5,7 \cdot v_{*}^{2}\right) \frac{L_{x}}{\bar{V}}}{1,339 \cdot\left(1+39,48 \cdot\left(\frac{f L_{x}}{\bar{V}}\right)^{2}\right)^{5 / 6}}
$$

siendo $\bar{V}$ la velocidad de viento media en $\mathrm{m} / \mathrm{s}, L_{x}$ la longitud integral longitudinal en $m$ y $v_{*}$ es la velocidad de corte que relaciona el esfuerzo cortante en el suelo con la densidad del aire $\sqrt{\frac{\tau_{p}}{\rho}}$ y se estima mediante:

$$
v_{*}=\frac{0,4 \cdot \bar{V}}{\log z / z_{0}}
$$

donde $z_{0}$ representa el coeficiente de rugosidad del terreno. Esta velocidad de corte puede relacionarse con la intensidad turbulenta según esta expresión $I_{v}=\frac{\sigma_{v}}{\bar{V}}=\frac{\sqrt{5,7 v_{*}}}{\bar{V}}$. Este modelo es adecuado para la estructura de turbulencia experimentada en un aerogenerador, particularmente en orografías complejas.

Otro modelo fue desarrollado en Davenport (1961) a partir de los valores medios de medidas obtenidas a diversas alturas. Su expresión es la siguiente:

$$
P S D_{\text {Davenport }}(f)=\sigma_{v}^{2} \cdot \frac{\frac{4}{6} \cdot f \cdot\left(\frac{L_{D a v}}{V}\right)^{2}}{\left(1+\left(\frac{f L_{D a v}}{\bar{V}}\right)^{2}\right)^{4 / 3}}
$$


CApítulo 2. Variabilidad y predictibilidad de la energía eólica y sus efectos sobre la red eléctrica

donde $L_{D a v}$ es la escala de longitud de turbulencia la cual es diferente de $L_{x}$ y se suele considerar como valor típico en el campo de la energía eólica $L_{\text {Dav }}=1200 \mathrm{~m}$.

De forma similar, Harris (1968) propone un modelo basado en el de Von Karman pero sustituyendo $L_{x}$ por otra definición de escala de longitud de turbulencia, $L_{\text {Harris }}$. La expresión resultante es la siguiente:

$$
P S D_{\text {Harris }}(f)=\sigma_{v}^{2} \cdot \frac{0,6 \cdot \frac{L_{\text {Harris }}}{\bar{V}}}{\left(2+\left(\frac{f L_{\text {Harris }}}{\bar{V}}\right)^{2}\right)^{5 / 6}}
$$

El modelo del espectro de potencia de Kaimal fue ajustado en base a datos experimentales recogidos con condiciones atmosféricas neutras en una localización llana en Kansas (EEUU), tal y como se explica en Kaimal et al. (1972). Este modelo solo es aplicable bajo condiciones neutras, las cuales coinciden en muchas ocasiones con fuertes vientos, ya que no se tiene en cuenta la convección. A pesar de estas limitaciones este modelo es muy usado ya que la condiciones descritas se aproximan a muchas aplicaciones como, por ejemplo en parques eólicos marinos. Además, la expresión que define el modelo es muy simple, lo que supone otra ventaja adicional. Esta expresión es la mostrada en 2.14 .

$$
P S D_{\text {Kaimal }}(f)=\frac{105 \cdot v_{*}^{2} \cdot z / \bar{V}}{(1+33 f \cdot z / \bar{V})^{5 / 3}}
$$

donde $z$ es la altura sobre el suelo en $m$. Este modelo, con pequeñas modificaciones ha sido utilizado en estudios más recientes como Veers (1988), Fégeant (2002), Rosas (2003) y Rauh y Peinke (2004).

La norma internacional IEC 61400-1, Committee et al. (2005), para los requerimientos en el diseño de aerogeneradores especifica una PSD del tipo de Kaimal

$$
P S D_{I E C}(f)=\sigma_{v}^{2} \cdot \frac{2 \cdot \frac{L_{1}}{\bar{V}}}{\left(1+6 \cdot f \cdot \frac{L_{1}}{\bar{V}}\right)^{5 / 3}}
$$

donde $L_{1}$ es la longitud de escala dada por:

$$
L_{1}= \begin{cases}5,67 \cdot z & z \leq 60 m \\ 340,2 m & z>60 m\end{cases}
$$

Solari (1987) propuso una modificación sobre los parámetros de los modelos de Kaimal y von Karman añadiendo una variable aleatoria en el modelo, como se muestra en 2.17 .

$$
P S D_{\text {Solari }}(f)=\frac{2,21 \cdot v_{*}^{2} \cdot \beta^{5 / 2} \cdot \frac{z}{\bar{V}}}{\left(1+3,31 \cdot f \cdot \beta^{3 / 2} \cdot \frac{z}{\bar{V}}\right)^{5 / 3}}
$$


donde $\beta$ viene dado por $\beta=\beta_{m}+\mu_{\beta} \cdot \Delta \beta$, siendo $\mu_{\beta}$ una variable aleatoria en el intervalo $[-1,1]$. $\beta_{m}$ y $\Delta \beta$ se calculan según 2.18 y 2.19 respectivamente.

$$
\begin{aligned}
& \beta_{m}= \begin{cases}7,5 & z_{0} \leq 0,03 \\
4,5-0,856 \log z_{0} & 0,03<z_{0} \leq 1,0 \\
4,5 & z_{0}>1,0\end{cases} \\
& \Delta \beta= \begin{cases}2,5 & z_{0} \leq 0,03 \\
2,0-0,143 \log z_{0} & 0,03<z_{0} \leq 1,0 \\
2,0 & z_{0}>1,0\end{cases}
\end{aligned}
$$

Los modelos descritos hasta el momento se desarrollaron principalmente para el diseño de aerogeneradores por lo que su rango de validez abarca unas escalas de tiempo entre $0,02 s$ y $600 s$. La variabilidad correspondiente a bajas frecuencias no está representada en los mismos. Debido a que los estudios de integración de la energía eólica incluyen las fluctuaciones de parques eólicos en escalas de tiempo que abarcan desde los 10 minutos hasta varias horas e incluso días, se presenta la necesidad de modelos que incluyan de forma válida las bajas frecuencias. Sørensen et al. (2008) propone un modelo que incluye la fluctuaciones de baja frecuencia basado en las mediciones registradas en la estación meteorológica de Høvsøre. Este modelo viene definido por la siguiente expresión:

$$
P S D_{B F}(f)=P S D_{B F, H o v}(f)+P S D_{I E C}(f)
$$

Se incluye una parte correspondiente al modelo de la IEC 61400-1 definido en 2.15 y otra parte encargada de modelizar las bajas frecuencias 2.21.

$$
P S D_{B F, H o v}(f)=\left(\alpha_{B F} \cdot \bar{V}+\beta_{B F}\right)^{2} \cdot \frac{\frac{z}{\bar{V}}}{\left(f \cdot \frac{z}{\bar{V}}\right)^{5 / 3} \cdot\left(1+100 \cdot f \cdot \frac{z}{\bar{V}}\right)^{5 / 3}}
$$

Los parámetros $\alpha_{B F}$ y $\beta_{B F}$ son estimados a partir de las mediciones realizadas. En el caso de la estación meteorológica de Høvsøre se estimaron los parámetros $\alpha_{B F} \simeq 0,0046$ y $\beta_{B F} \simeq 0$ según Sørensen et al. (2007). En Vigueras-Rodríguez (2008) se definieron estos parámetros para otras dos localizaciones, los parques eólicos marinos de Nysted y Horns Rev. En ambas localizaciones se obtuvo un $\alpha_{B F}=0$ mientras que $\beta_{B F} \simeq 0,041 \mathrm{~m} / \mathrm{s}$ en Nysted y $\beta_{B F} \simeq 0,037 \mathrm{~m} / \mathrm{s}$ en Horns Rev.

\subsubsection{Coherencia espectral de la velocidad del viento}

La coherencia de la velocidad de viento es un parámetro importante ya que muestra la correlación entre las velocidades de viento en diferentes localizaciones. La función de la coherencia $\gamma$ se define como: 
CApítulo 2. Variabilidad y predictibilidad de la energía eólica y sus

$$
|\gamma(f)|=\frac{S_{i j}(f)}{\sqrt{S_{i i}(f) \cdot S_{j j}(f)}}
$$

donde $S_{i i}$ y $S_{j j}$ son las auto densidades espectrales de potencia en las localizaciones $i$ y $j$ respectivamente, y $S_{i j}$ es la función de densidad espectral de potencia cruzada entre las localizaciones $i$ y $j . S_{i j}$ puede ser descompuesta en una parte real, llamada coespectro $C o_{i j}(f)$, y una parte imaginaria, llamada espectro de cuadratura $Q u_{i j}(f)$. Así la expresión 2.22 se puede reescribir como:

$$
|\gamma(f)|=\frac{C o_{i j}(f)+i Q u_{i j}(f)}{\sqrt{S_{i i}(f) \cdot S_{j j}(f)}}
$$

El coespectro y el espectro de cuadratura representan las contribuciones simultáneas y con un cierto desfase respectivamente de la covarianza de dos series temporales. Si las series temporales están perfectamente correlacionadas a una cierta frecuencia $f_{0}$, el valor de coespectro será de $C o\left(f_{0}\right)=1$ y el espectro de cuadratura será de $Q u\left(f_{0}\right)=0$. Por el contrario, si en dos series idénticas una está retrasada medio ciclo con respecto a la otra, los valores de coespectro y espectro de cuadratura serán respectivamente $C o(f)=-1$ y $Q u(f)=0$. Finalmente, si una series está retrasada un cuarto de ciclo con respecto a la otra los valores serán $C o(f)=0$ y $Q u(f)= \pm 1$, dependiendo el signo de cuál es la serie retrasada. El caso particular del espectro de Taylor consiste en un valor de coherencia $|\gamma(f)|=1$ y un valor de espectro de cuadratura $i Q u(f)<0$ indicando el retraso.

La correlación del viento entre dos localizaciones diferentes depende de la velocidad media del viento, la distancia entre las posiciones y el rango de frecuencias estudiadas. Así, las velocidades de viento en emplazamientos cercanos están altamente correlacionadas, pero está correlación se reduce a medida que aumenta la distancia entre los puntos de medida. Del mismo modo la turbulencia en la velocidad de viento está altamente correlacionada en la parte de baja frecuencia y disminuye a medida que la frecuencia aumenta. En Davenport (1961) se estableció por primera vez una relación entre las variables descritas y la coherencia espectral. Esta relación es de tipo exponencial. La expresión que incluye esta relación se muestra en 2.24.

$$
|\gamma(f)|=e^{-k \frac{f}{\bar{V}} \cdot d_{i j}}
$$

donde $d_{i j}$ es la distancia entre las localizaciones $i$ y $j$, siendo $k$ es una constante denominada como el factor de decaimiento. El factor de decaimiento ha sido originalmente expresada como una constante igual a 7,7 Davenport (1961). Más tarde Frost et al. (1978) modificaron su valor a 7,5. Las mediciones en otros emplazamientos, sin embargo, revelan un valor diferente que está en el intervalo de 2 a 27 que está principalmente relacionada con la 
relación entre la distancia entre los dos puntos y la altura de las mediciones $d_{i j} / h_{i j}$ tal y como se presenta en Solari (1987).

En el caso de un parque eólico, el ángulo formado por la dirección del viento y la recta que une las dos localizaciones medidas, denominado ángulo de incidencia $\alpha_{i j}$, tiene una gran importancia en la función de la coherencia. Así, en Schlez y Infield (1998) se combinan las expresiones de la coherencia longitudinal $a_{\text {long }}$ y la coherencia lateral $a_{\text {lat }}$ para formular el factor de decaimiento en función del ángulo de incidencia, dando como resultado la expresión 2.25 .

$$
k=\sqrt{\left(a_{\text {long }} \cos \alpha_{i j}\right)^{2}+\left(a_{\text {lat }} \sin \alpha_{i j}\right)^{2}}
$$

Se proponen como valores de los factores longitudinal y lateral los valores siguientes:

$$
\begin{aligned}
& a_{\text {long }}=(15 \pm 5) \cdot I_{v} \\
& a_{\text {lat }}=(17,5 \pm 5) \cdot \frac{I_{v}}{\bar{V}}
\end{aligned}
$$

siendo $I_{v}$ la intensidad turbulenta. Estos valores se estudiaron a partir de medidas en estaciones meteorológicas, a una altura de $18 \mathrm{~m}$, y a distancias de hasta 102 m. En Sørensen et al. (2002) se utilizan distancias de hasta 1 $\mathrm{km}$ para analizar la expresión de decaimiento exponencial, tanto el termino seno como el coseno, con el objetivo de establecer un modelo de potencia eólica en emplazamientos marinos.

Por su parte, Nanahara et al. (2004b) calculó los factores de decaimiento para emplazamientos situados a unas distancias de entre 100 y $1700 \mathrm{~m}$ tanto en terreno llano como en terreno complejo. Así se establece una considerable dependencia de los factores de decaimiento según la dirección relativa del viento.

La norma ICE 61400-1, Committee et al. (2005), establece la coherencia entre dos puntos $i$ y $j$ separados por una distancia $d_{i j}$ en el área del rotor de un aerogenerador, como la siguiente expresión:

$$
\left|\gamma_{I E C}\right|(f)=e^{-A \cdot \sqrt{\left(\frac{d_{i j} \cdot f}{\bar{V}}\right)^{2}+\left(0,12 \cdot \frac{d_{i j}}{L_{1}}\right)^{2}}}
$$

Según la IEC $61400-1$ se recomienda $A \approx 12$. Por otra parte, Saranyasoontorn et al. (2004) lo ajusta como $A \approx 9,7$ y Frandsen et al. (2007) recomienda $A \approx 5$.

Ampliando lo anterior, en Rosas (2003), la componente de correlación de la velocidad de viento se estima según el modelo de Davenport y el factor de decaimiento presentado por Schlez e Infield, demostrando que su valor 
CApítulo 2. Variabilidad y predictibilidad de la energía eólica y sus 24 efectos sobre la red eléctrica

para distancias superiores a $100 \mathrm{~m}$ es inferior a 20 en las frecuencias correspondientes al efecto sombra de la torre (entre $0,5 \mathrm{~Hz}$ y $2 \mathrm{~Hz}$ ). De hecho, la coherencia dentro de las dimensiones habituales de un parque eólico es baja para oscilaciones más rápidas que $0.001 \mathrm{~Hz}$, como se describe en Sørensen et al. (2008). Por lo tanto, para fluctuaciones con frecuencias superiores a un ciclo cada 10 minutos existe una baja correlación entre las turbinas de un parque eólico.

Debido a la falta de modelos de coherencia espectral apropiados capaces de cubrir las distancias y las características de grandes parques eólicos, Vigueras-Rodríguez (2008) propone un modelo basado en los datos disponibles del parque eólico marino Nysted. Se propone un factor de decaimiento lateral $a_{\text {lat }}$ variable en función de la velocidad media $\bar{V}$ y la distancia $d_{i j}$, resultando las siguientes expresiones:

$$
\begin{gathered}
\gamma\left(d_{i j}, \bar{V}, \alpha_{i j}, f\right)=e^{\sqrt{\left(a_{\text {long }} \cos \alpha_{i j}\right)^{2}+\left(a_{\text {lat }}\left(d_{i j}, \bar{V}\right) \sin \alpha_{i j}\right)^{2}} \frac{d_{i j} \cdot f}{\bar{V}}} \\
a_{\text {long }} \approx 4,5 \\
a_{\text {lat }}\left(d_{i j}, \bar{V}\right) \approx 466(s) \cdot \frac{\bar{V}}{d_{i j}}+4,2
\end{gathered}
$$

En caso de zonas más amplias, se han realizado varios estudios últimamente debido a su importancia en la caracterización de las fluctuaciones eólicas, tanto para la integración de la energía eólica como para mejorar la predicción de la misma. En Woods et al. (2011) se construyen expresiones de coherencia similares a las de Schlez e Infield para proponer modelos de coespectro y espectro de cuadratura basado en un extenso conjunto de mediciones de torres meteorológicas separadas entre 1 y $30 \mathrm{~km}$ en el Sureste de Australia. Las expresiones obtenidas son más complejas e incluyen el ajuste de parámetros adicionales. Larsén et al. (2012) calcula diferentes parámetros espectrales, incluyendo la coherencia espectral, para seis torres meteorológicas situadas en el Mar del Norte y en el Mar Báltico con distancias entre ellos de 2.13 a $12.42 \mathrm{~km}$. Con los mismos datos, Vincent et al. (2012) se centra en caracterizar el coespectro y el espectro de cuadratura, ajustando las ecuaciones siguientes:

$$
\begin{aligned}
& C o\left(\alpha, d_{i j}, \bar{V}, f\right)=e^{a(\alpha) \cdot \frac{f \cdot d_{i j}}{\bar{V}}} \cdot \cos \left(b(\alpha) \frac{f \cdot d_{i j}}{\bar{V}}\right) \\
& Q u\left(\alpha, d_{i j}, \bar{V}, f\right)=e^{a(\alpha) \cdot \frac{f \cdot d_{i j}}{\bar{V}}} \cdot \sin \left(b(\alpha) \frac{f \cdot d_{i j}}{\bar{V}}\right)
\end{aligned}
$$

siendo 


$$
\begin{gathered}
a(\alpha)=1,8 \cdot \cos \left(\frac{2 \cdot \pi \cdot \alpha}{180}\right)-5,9 \\
b(\alpha)=2 \cdot \pi \cdot \cos \left(\frac{\pi \cdot \alpha}{180}\right)
\end{gathered}
$$

\subsubsection{Análisis temporal y probabilístico de la velocidad del viento}

El término fluctuaciones es ampliamente utilizado en la literatura cuando se refiere a las variaciones por encima y por debajo de los valores medios, tal como se define en la ecuación 2.36 :

$$
U(t)=u_{\text {ave }}+u
$$

donde $U(t)$ es una cantidad variable en el tiempo, $u_{\text {ave }}$ representa el valor medio y $u$ representa la fluctuación de respecto al valor medio. Aunque estadísticamente, la definición usada en 2.36 es la usual para describir la componente fluctuante en una sola variable, esa definición no es la forma más útil para describir la variabilidad de la generación eólica con respecto a la operación del sistema eléctrico, ya que la preocupación aquí radica en la perturbación de las condiciones de operación actuales del instante "t". Por lo tanto, se puede establecer una nueva expresión para caracterizar la velocidad de viento con variaciones relativamente lentas en el tiempo como:

$$
U(t+\Delta t)=U(t)+\Delta U(t)
$$

donde $U(t)$ representa el valor de la velocidad de viento en el instante " $t$ ", $U(t+\Delta t)$ es el valor de la velocidad de viento en el instante " $\mathrm{t}+1$ " y $\Delta U(t)$ indica la variación entre dos instantes consecutivos.

En el caso de la velocidad de viento, los principales atributos de interés a la hora de analizar una serie temporal son la media, la desviación estándar y las variaciones en diferentes escalas de tiempo. El valor promedio no es sensible al periodo de la serie temporal, por el contrario, depende únicamente de la localización elegida y de la longitud de la serie. En cambio, los valores de las variaciones de la velocidad de viento en una localización si vienen caracterizados por el periodo entre dos valores promedio $(\Delta t)$ elegido, además de por el número de muestras considerado en la serie temporal y por las características propias del viento en ese lugar. Debido a esta dependencia de la longitud de la serie, es necesario disponer de una campaña de medidas lo suficientemente larga y definir los periodos necesarios, para poder estudiar el recurso eólico en cualquier estudio de integración en la red de la energía eólica.

En la figura 2.3 se muestra la velocidad de viento para diferentes periodos en la localización del parque WF18. Así mismo se muestran las variaciones de 
CAPÍtulo 2. Variabilidad y predictibilidad de la energía eólica y sus efectos sobre la red eléctrica

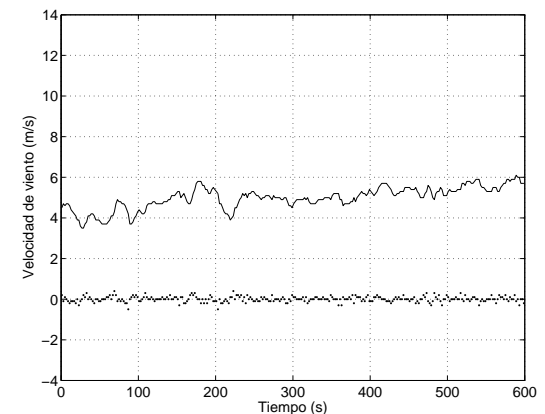

(a) 2 segundos

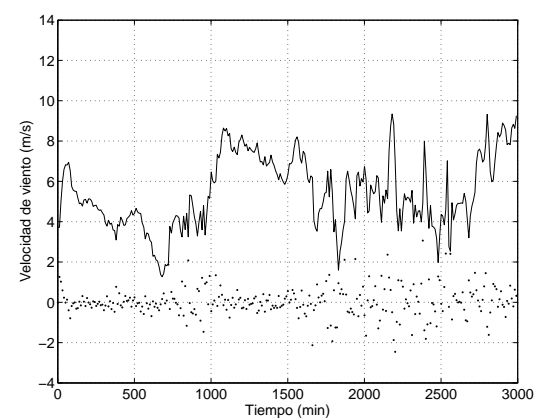

(c) 10 minutos

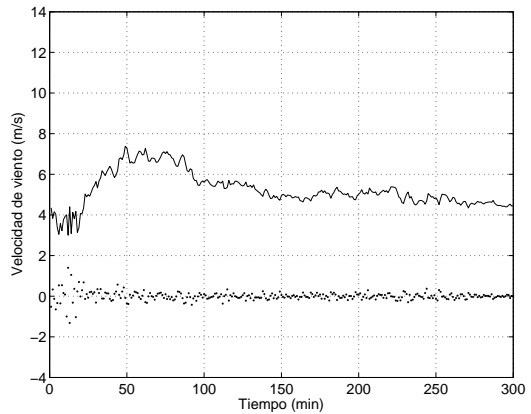

(b) 1 minuto

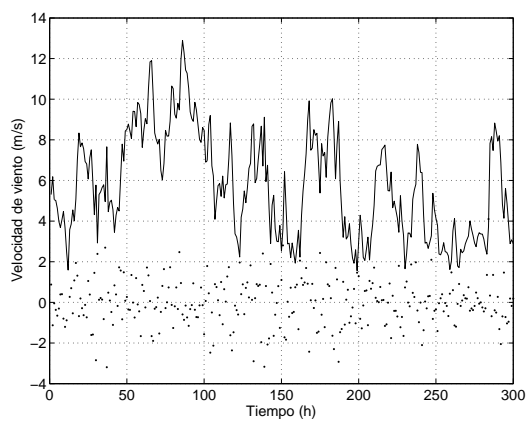

(d) 1 hora

Figura 2.3: Velocidad de viento y variación de la misma para diversos periodos de medias consideradas en la localización del parque WF18

la velocidad de viento para dos periodos consecutivos. El número de muestras es idéntico para las cuatro representaciones para poder analizar el efecto del periodo en las variaciones obtenidas. Así, de estas representaciones se puede extraer que, manteniendo constante el número de muestras, cuanto mayor es el periodo considerado mayor es la variabilidad de la velocidad de viento. En el caso de un periodo de 2 segundos las variaciones son las más pequeñas y la velocidad de viento tiene una apariencia más suave. Por el contrario, en el caso de un periodo de 1 hora se aprecian grandes variaciones, llegando a $\pm 4 \mathrm{~m} / \mathrm{s}$.

En una serie temporal, el parámetro más utilizado para el análisis de la variabilidad es la desviación estándar $\left(\sigma_{\Delta V}\right)$ de las variaciones, Holttinen (2005b). El valor de esta desviación estándar corresponde con la ecuación 2.38 .

$$
\sigma_{\Delta V}=\sqrt{\frac{\sum_{i=1}^{n}\left(x_{i}-\mu\right)^{2}}{n}}
$$

donde $x_{i}$ es el valor i de la serie temporal, $\mu$ es el valor medio de la serie 


\begin{tabular}{ccccc}
\hline- & $\begin{array}{c}2 \text { segundos } \\
{[\mathrm{m} / \mathrm{s}]}\end{array}$ & $\begin{array}{c}1 \text { minuto } \\
{[\mathrm{m} / \mathrm{s}]}\end{array}$ & $\begin{array}{c}10 \text { minutos } \\
{[\mathrm{m} / \mathrm{s}]}\end{array}$ & $\begin{array}{c}1 \text { hora } \\
{[\mathrm{m} / \mathrm{s}]}\end{array}$ \\
\hline Max. velocidad & 16.8 & 15.7 & 13.5 & 12.9 \\
Min. velocidad & 0.10 & 0.12 & 0.42 & 0.70 \\
Dev. estándar & 2.55 & 2.51 & 2.46 & 2.41 \\
Media velocidad & 5.71 & 5.70 & 5.70 & 5.69 \\
\hline
\end{tabular}

Tabla 2.1: Datos estadísticos básicos de la velocidad de viento en el parque WF18 para distintos intervalos de medias temporales durante el mes de Marzo de 2012

temporal y $n$ es el número de valores de la serie.

En el caso de una distribución de probabilidad normal, la desviación estándar $\sigma$ es una medida que indica que el $68 \%$ de las medidas se encuentran dentro de un rango de $\pm \sigma$ del valor medio. Tomando un rango de $\pm 3 \sigma$ se cubre un $99 \%$ de los datos y aumentando el rango a $\pm 4 \sigma$ abarca el 99.99 $\%$ de todas las variaciones.

En la tabla 2.1 se muestran los principales valores estadísticos de la velocidad de viento para el parque WF20 durante el mes de Marzo de 2012. Se aprecia que el rango existente entre los valores límite se estrecha según se aumenta el intervalo. Del mismo modo, la desviación estándar se reduce muy ligeramente al aumentar el periodo, manteniéndose la media prácticamente constante. Estos parámetros se pueden considerar independientes del periodo elegido, tal y como se ha descrito anteriormente.

En el caso de las variaciones de la velocidad de viento, el periodo condiciona los parámetros obtenidos. La tabla 2.2 muestra los parámetros de las variaciones para los mismos datos que en la tabla 2.1. Se puede apreciar que la desviación estándar de las variaciones aumenta considerablemente según crece el periodo. Por otra parte, las variaciones extremas más altas están localizadas en el periodo de 1 minuto. Esto se debe al pico micrometeorológico descrito en el espectro de Van der Hoven (figura 2.1), el cual se centra en una frecuencia de aproximadamente 1 ciclo/min. Los periodos de 10 minutos y 1 hora están dentro del "hueco espectral" y las variaciones extremas son más reducidas que para 1 minuto. En el caso de 2 segundos se presentan los extremos más reducidos de los casos estudiados. Estas conclusiones se pueden transferir a otras localizaciones tal y como se indica en Wan (2005).

\subsubsection{Características diarias y estacionales de la velocidad del viento}

El viento es impulsado por los frentes meteorológicos y un patrón diario causado por el sol, por lo que, dependiendo de si un efecto u otro de ellos 
CApítulo 2. Variabilidad y predictibilidad de la energía eólica y sus efectos sobre la red eléctrica

\begin{tabular}{ccccc}
\hline- & $\begin{array}{c}2 \text { segundos } \\
{[\mathrm{m} / \mathrm{s}]}\end{array}$ & $\begin{array}{c}1 \text { minuto } \\
{[\mathrm{m} / \mathrm{s}]}\end{array}$ & $\begin{array}{c}10 \text { minutos } \\
{[\mathrm{m} / \mathrm{s}]}\end{array}$ & $\begin{array}{c}1 \text { hora } \\
{[\mathrm{m} / \mathrm{s}]}\end{array}$ \\
\hline Max. variación positiva & 2.50 & 6.16 & 3.53 & 4.10 \\
Max. variación negativa & -2.00 & -5.29 & -4.26 & -3.19 \\
Dev. estándar & 0.17 & 0.60 & 0.65 & 1.15 \\
\hline
\end{tabular}

Tabla 2.2: Datos estadísticos básicos de las variaciones de la velocidad de viento en el parque WF18 para distintos intervalos de medias temporales durante el mes de Marzo de 2012

predominan, existirá un patrón diurno significativo en la producción o no. En Europa hay una tendencia a que los vientos empiecen a soplar por la mañana y calmarse por la noche, tal y como ocurre en Irlanda, según Hurley y Watson (2002) y en Alemania, según Ensslin et al. (2000)). En el norte de Europa esto es más pronunciado durante el verano, debido a que el patrón de vientos térmicos inducidos es más pronunciado en esta época, Sinden (2007).

En Europa existe una premisa de variación estacional del viento que consiste en una mayor producción en invierno que en verano. La producción durante los meses de verano representa el 60-80\% de la media anual, mientras que la producción durante los meses de invierno representa entre el $110 \%$ y el $150 \%$ de la media anual, Holttinen (2005a).

Esta variación estacional y diaria del viento ha de ser estudiada en profundidad para un área concreta para ver cómo se adecua a la demanda existente.

\subsubsection{Función de autocorrelación (ACF) de la velocidad del viento}

La ACF de un proceso estocástico se describe como la correlación entre los valores del proceso en diferentes puntos en el tiempo. La ACF se encarga de medir la persistencia cronológica del proceso. La ACF se calcula según la ecuación 2.39, siendo $v(t)$ la serie temporal de la velocidad de viento dada en el instante $t$.

$$
A C F(t, t+k)=\frac{E[(v(t)-\mu)(v(t+k)-\mu)]}{\sigma^{2}}
$$

En el caso de la velocidad de viento existe pronunciada persistencia en el tiempo, tal y como señalan Huang y Chalabi (1995). En Brett y Tuller (1991) se demuestra que la función de autocorrelación de los datos horarios de velocidad del viento presenta una rápida caída de tipo exponencial en intervalos cortos (menos de $15 \mathrm{~h}$ ) seguida por una caída más lenta en intervalos medios. Para intervalos largos (más de 48 horas) existe una periodicidad, como resultado de componentes determinísticos como son los ciclos solares diarios y anuales de calentamiento de la atmósfera. 


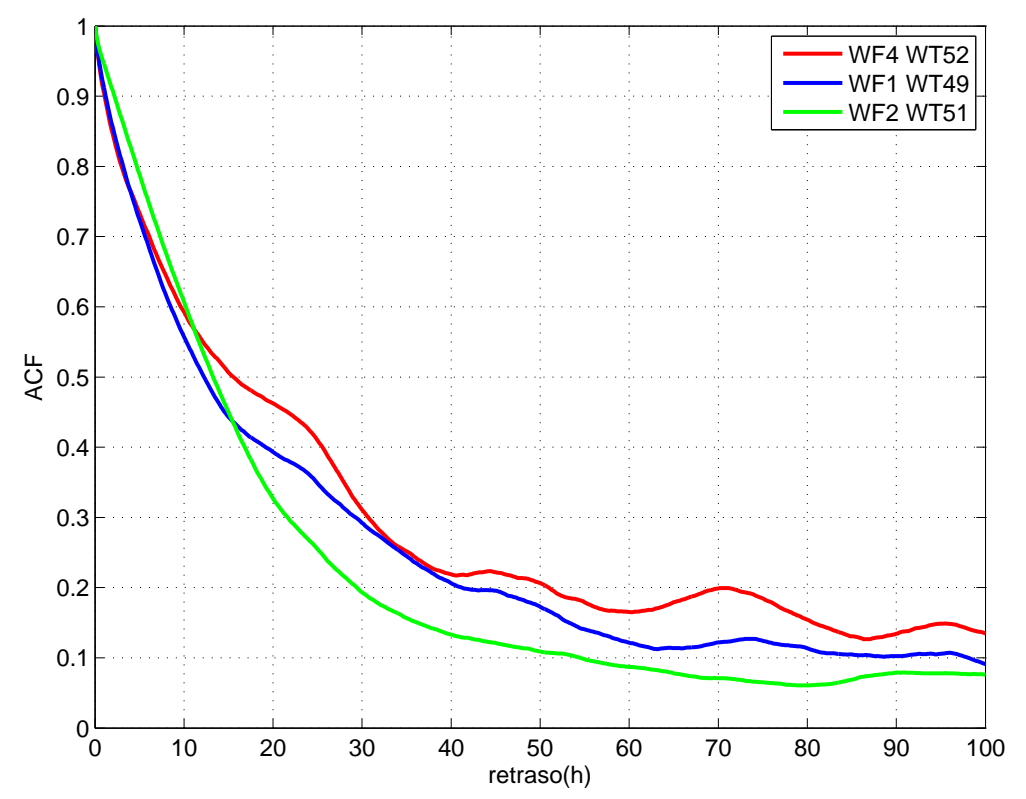

Figura 2.4: ACF de la velocidad de viento para tres localizaciones distintas

La figura 2.4 representa la función de autocorrelación de tres localizaciones distintas. Se aprecia que la periodicidad típica debida al ciclo solar diario en las localizaciones WF4 WT52 y WF1 WT49, mientras que en el emplazamiento WF2 WT51 es prácticamente inexistente. Por otra parte la caída rápida presente en los intervalos más cortos $(<10 h)$ es menos acentuada en el emplazamiento WF2 WT51.

Este grado de correlación entre los sucesivos valores horarios de velocidad del viento indica que la velocidad del viento puede ser modelada de forma razonable por un proceso de autorregresivo.

\subsubsection{Función de distribución de probabilidad (PDF) de la ve- locidad del viento}

La velocidad de viento medida en una localización durante un periodo relativamente largo sigue una distribución Weibull. Así, para representaciones teóricas, los parámetros de la distribución Weibull, el factor de escala $A$ y el factor de forma $k$ son extraídos del ajuste a la distribución de los datos medidos, resultando la función de probabilidad de la velocidad de viento siguiente:

$$
P D F(v)=\frac{k}{A} \cdot\left(\frac{v}{A}\right)^{k-1} \cdot e^{-\left(\frac{v}{A}\right)^{k}}
$$

En la figura 2.5 se proponen ejemplos de PDF de la velocidad de vien- 
Capítulo 2. Variabilidad y predictibilidad de la energía eólica y sus efectos sobre la red eléctrica

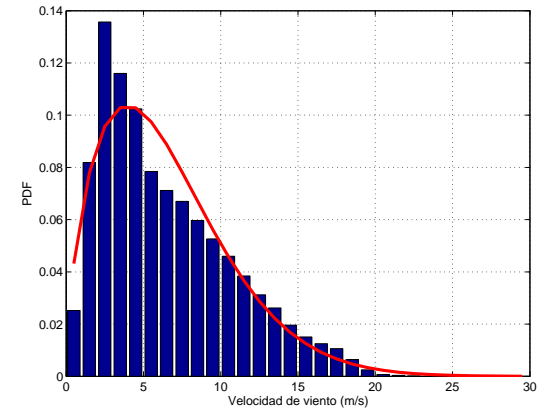

(a) WF4 WT52

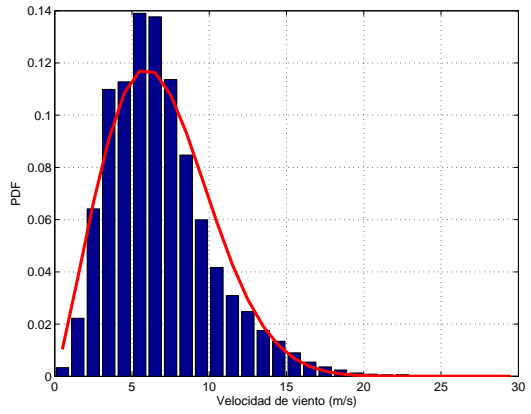

(b) WF1 WT49

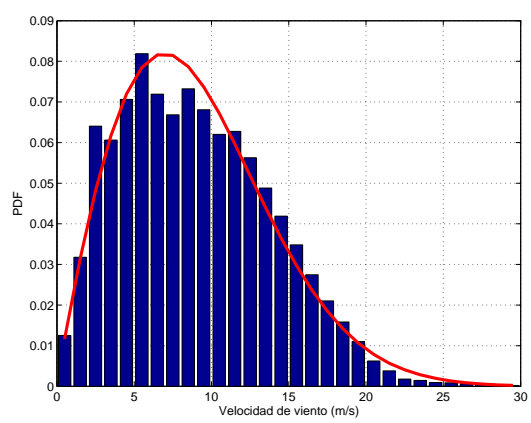

(c) WF2 WT51

Figura 2.5: Función de distribución de probabilidad real y ajustada a una distribución Weibull para los 3 emplazamientos señalados

to para 3 localizaciones distintas y las distribuciones Weibull ajustadas a esos datos. Los parámetros de las distribuciones Weibull correspondientes se muestran en la tabla 2.3. Se puede apreciar que los valores $A$ y $k$ caracterizan los valores de velocidad de viento más predominantes y la frecuencia con la que aparecerán valores extremos. De esta forma, cuanto mayor sea el parámetro $A$ existirá una mayor probabilidad de que se produzcan valores más extremos.

\begin{tabular}{cccc}
\hline- & WF4 WT52 & WF1 WT49 & WF2 WT51 \\
\hline Factor de escala $A$ & 7.3388 & 7.7939 & 10.208 \\
Factor de forma $k$ & 1.5972 & 2.2046 & 1.921 \\
\hline
\end{tabular}

Tabla 2.3: Parámetros de las distribuciones Weibull ajustadas en la figura 2.5 


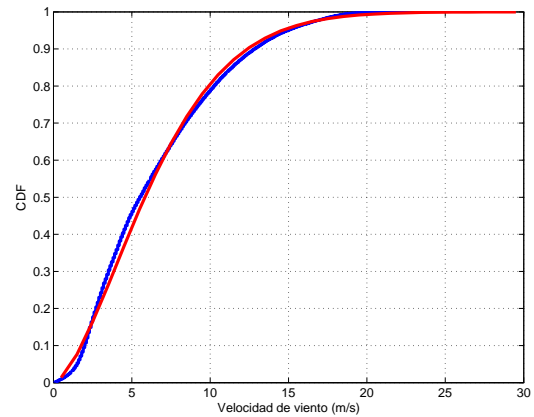

(a) WF4 WT52

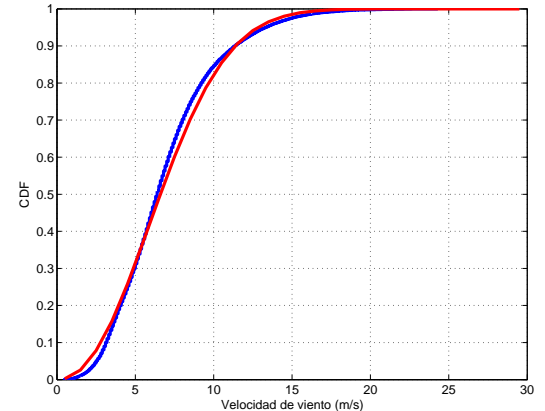

(b) WF1 WT49

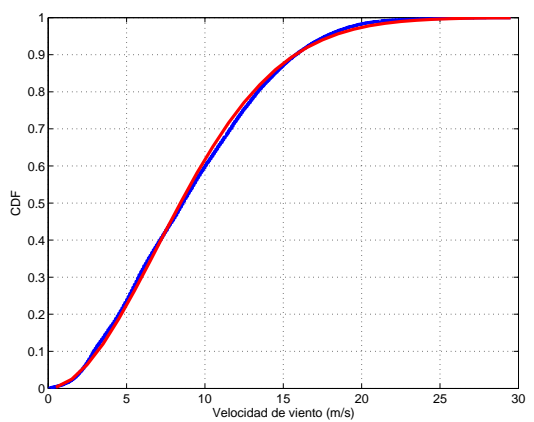

(c) WF2 WT51

Figura 2.6: Función de distribución acumulada real y ajustada a una distribución Weibull para 3 emplazamientos señalados

\subsubsection{Función de distribución acumulada (CDF) de la velocidad del viento}

La distribución acumulada es una función que establece la probabilidad que existe para que una variable aleatoria de encontrar un valor menor o igual que un valor dado. La forma principal de representar esta función es a partir de una curva de duración.

En el caso de la velocidad de viento, al ajustar el PDF a una distribución Weibull, la función de distribución acumulada se puede aproximar a la siguiente expresión:

$$
C D F(v)=1-e^{-\left(\frac{v}{A}\right)^{k}}
$$

De forma análoga a la figura 2.5, en la figura 2.6 se proponen CDF reales de 3 emplazamientos y se comparan con las distribuciones Weibull acumuladas según la ecuación 2.41 . 
CAPítulo 2. Variabilidad y predictibilidad de la energía eólica y sus efectos sobre la red eléctrica

\subsection{Análisis de fluctuaciones de potencia eólica}

En el caso de un aerogenerador, la causa principal de las fluctuaciones de potencia de salida del mismo es la variación natural de la velocidad de viento. Aun así existen otras fuentes de la variabilidad de la potencia eólica, tanto en periodos cortos (de segundos a minutos) como en periodos largos (de horas a días o incluso estaciones). El tipo de aerogenerador y los algoritmos de control del mismo, afectan a la variabilidad de la potencia entregada a la red en periodos cortos. Las palas de un aerogenerador de inducción tipo 1 experimentan el efecto de sombra de la torre cada vez que una pala pasa por la torre. Esto hace que la que la potencia de salida fluctúe a causa de este efecto. Los aerogeneradores tipo 3 y tipo 4 están equipados con electrónica de potencia y poseen un rango razonable de variación de la velocidad de giro, por lo que el efecto de la torre puede ser enmascarado por el control del convertidor de potencia.

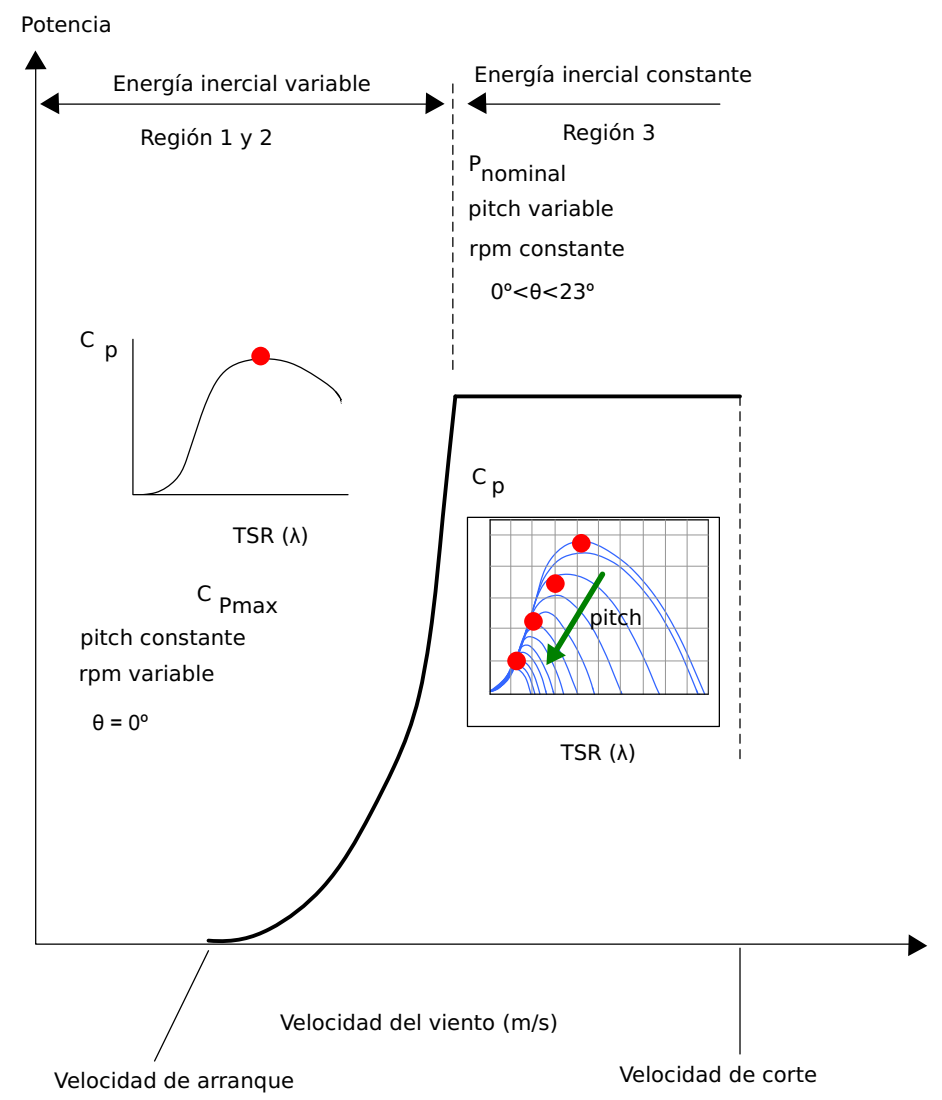

Figura 2.7: Curva de potencia de un aerogenerador de velocidad variable 
Por otra parte, la potencia de salida de un aerogenerador viene caracterizada por su curva de potencia. La curva se puede dividir en 4 zonas principales determinadas por la velocidad de arranque, la limitación de potencia al llegar a la potencia nominal y la velocidad de corte. En la figura 2.7 se muestra la curva de potencia típica de un aerogenerador de velocidad variable. Por lo general, la velocidad de viento a la que opera el aerogenerador se divide en 4 regiones. En la región 1 (zona por debajo de la velocidad de arranque) el aerogenerador permanece inoperativo debido a que la velocidad de viento es muy pequeña. En la región 2 (zona de operación a carga parcial) la operación es óptima debido a que el aerogenerador opera con velocidad variable a una ángulo de pala (pitch) constante, normalmente $0^{\circ}, \mathrm{y}$ su coeficiente de potencia $C_{p}$ es máximo. Mientras se opera a carga parcial, la potencia de salida varia como el cubo de las variaciones de la velocidad de viento. Una vez que se supera la velocidad nominal (región 3) se ha de limitar la velocidad rotacional mediante el control del ángulo de pala (pitch) para mantener la velocidad del rotor en condiciones estables y limitar las cargas mecánicas sufridas por los elementos estructurales (torre, palas y multiplicadora). De esta forma la potencia de salida se limita al valor nominal manteniéndose constante para toda la región. Por último, si la velocidad de viento supera la velocidad de corte, la potencia varia del $100 \%$ (potencia nominal) al $0 \%$.

En resumen, la potencia de salida solo varía cuando la velocidad de viento está por debajo del valor nominal o cuando se supera la velocidad de corte. El rango de cambio $\frac{d P}{d t}$ puede ser positivo o negativo dependiendo de la dirección del cambio en la velocidad de viento. Para velocidades por encima de la velocidad nominal el cambio será nulo $\frac{d P}{d t}=0$ accionando el control del ángulo de pala.

La conversión de potencia eólica a potencia eléctrica de salida produce un filtrado y suavizado sobre la variabilidad con respecto a la presente en la velocidad de viento. Así, los parámetros probabilísticos (PDF y CDF) y de autocorrelación $(\mathrm{ACF})$ varíen notablemente con respecto a lo establecido para la velocidad de viento. La autocorrelación de la potencia entregada por un aerogenerador presenta una mayor caída en intervalos cortos que la que presenta la velocidad de viento y la periodicidad debida al ciclo solar diario ocurre de forma similar a lo descrito para la velocidad de viento. Esto se muestra en la figura 2.8 .

En cuanto a la PDF, la distribución ya no se ajusta a una distribución Weibull. Las mayores probabilidades se localizan sobre la potencia nula o sobre la potencia nominal. La principal razón es el amplio rango de velocidades involucradas en cada caso, región 1 para la potencia nula y región 3 para la potencia nominal. La figura 2.9 muestra 3 ejemplos de distribuciones en los mismos emplazamientos que los mostrados en la figura 2.5.

En el caso de un parque eólico, la potencia total es la suma de la potencia de salida de cada uno de los aerogeneradores. Las fluctuaciones de potencia 
CAPítulo 2. Variabilidad y predictibilidad de la energía eólica y sus efectos sobre la red eléctrica

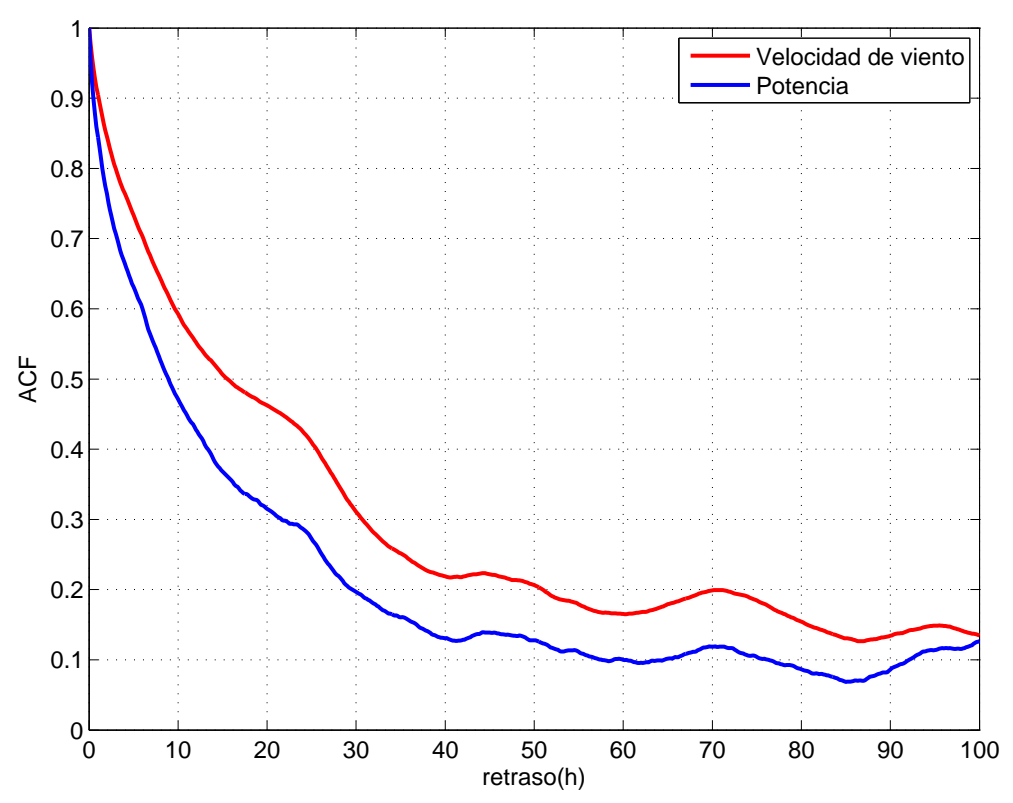

Figura 2.8: Comparación entre la ACF de la velocidad de viento y de la potencia de salida de un aerogenerador

activa difieren entre los diferentes parques eólicos debido principalmente a las condiciones meteorológicas, así como la localización, orografía y distribución de los mismos.

En cuanto a la localización marina o terrestre, en los parques terrestres ("onshore") las fluctuaciones son menos acentuadas que en los marinos ("offshore"). Esto se debe a dos razones, principalmente. La primera es que los aerogeneradores esta dispuestos en una zona más pequeña en los parques marinos por lo que sus fluctuaciones tienen una mayor correlación. Las condiciones meteorológicas marinas también contribuyen a la mayor variabilidad en los parques "offshore". Esta diferencias se analizan en Martín-Martínez et al. (2009a).

La orografía de la zona en la que se emplaza el parque eólico tiene un valor crítico en los valores de coherencia entre los distintos aerogeneradores. De este modo, según la dirección de la velocidad de viento tiene un peso importante sobre la variabilidad de la potencia del parque é́lico como se indica en Nanahara et al. (2004a) y Nanahara et al. (2004b).

Las fluctuaciones de la producción de un parque eólico también están influenciadas por el tamaño del parque y la colocación de los aerogeneradores. En Spahic y Balzer (2005), se detalla la reducción de la variabilidad de la potencia de un parque eólico al incrementar su tamaño y el número de aerogeneradores del mismo. 


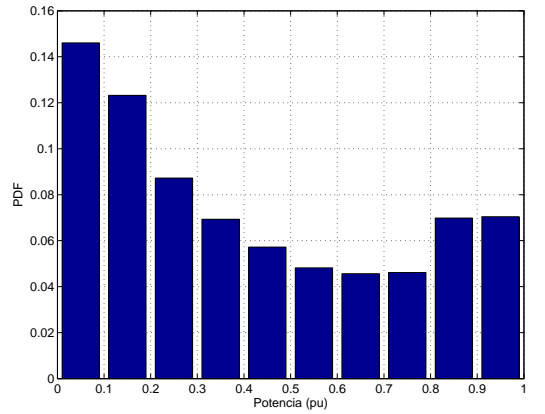

(a) WF4 WT52

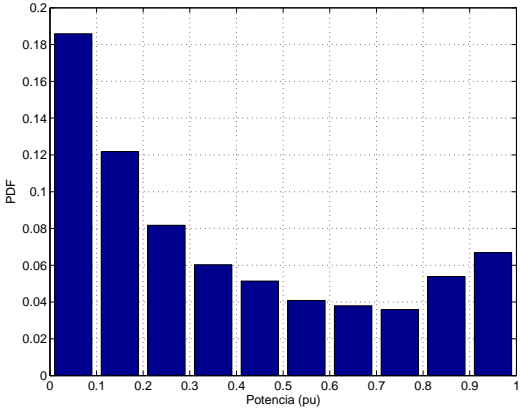

(b) WF1 WT49

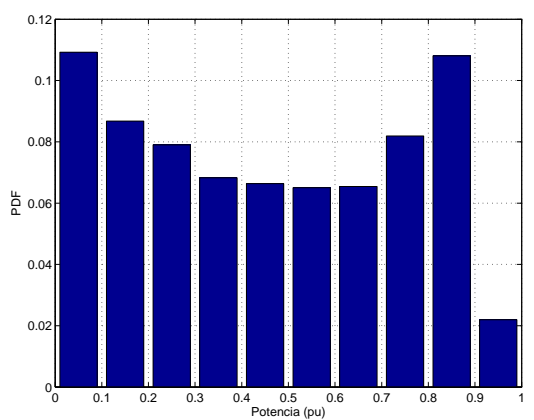

(c) WF2 WT51

Figura 2.9: Función de distribución de probabilidad real de la potencia generada por 3 aerogeneradores en emplazamientos diferentes

\subsubsection{Medida y caracterización de las fluctuaciones de po- tencia eólica}

En el caso concreto de la potencia eólica generada, en Parsons et al. (2004) se propone el uso de las rampas para la caracterización de las fluctuaciones. Esta se define como el cambio en la potencia entre dos periodos consecutivos en valores medios. Esto queda reflejado en la siguiente ecuación:

$$
P_{r a m p}=\frac{\overline{P_{n+1}(t)}-\overline{P_{n}(t)}}{T}
$$

siendo $\overline{P_{n}}$ la potencia media producida en el intervalo $n, \overline{P_{n+1}}$ la potencia media producida en el intervalo $n+1$ y $T$ el tiempo entre ambos intervalos. Esta definición es similar a la efectuada en 2.37 para la velocidad de viento.

Las rampas pueden ser positivas, cuando la potencia entregada aumenta de un instante al siguiente, o negativas cuando la generación disminuye de un instante al siguiente.

De forma estadística, la variabilidad de la potencia eólica se suele representar mediante la desviación estándar de las rampas. Este parámetro 
Capítulo 2. Variabilidad y predictibilidad de la energía eólica y sus 36 efectos sobre la red eléctrica

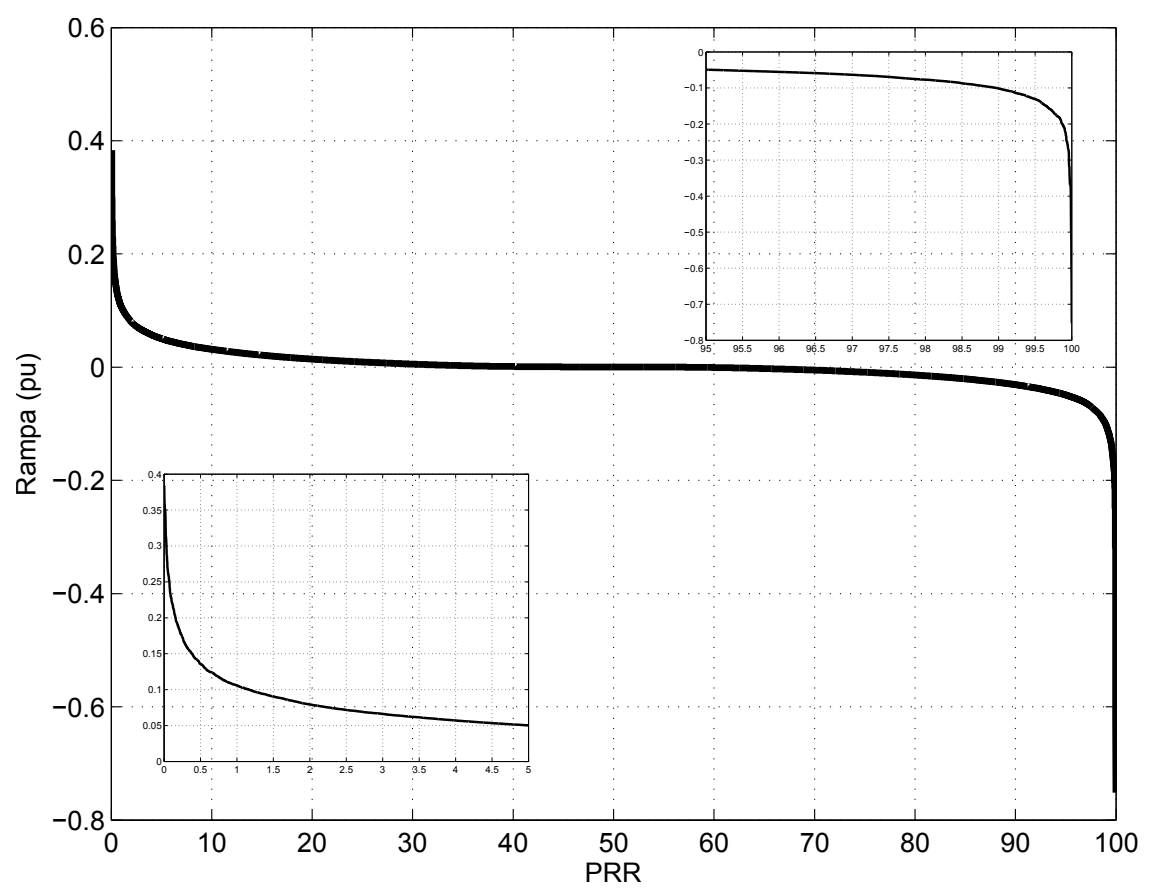

Figura 2.10: Distribución acumulada de rampas reales para el parque eólico WF1

es usado en diferentes estudios como Holttinen et al. (2008) y Bouffard y Galiana (2008). Así se considera que la distribución de las rampas sigue una distribución normal y es simétrica respecto de 0 . Esto no ocurre en la mayoría de la ocasiones tal y como se puede apreciar en la figura 2.10. Adicionalmente, en la figura 2.11 se muestra una comparación entre las curvas de duración real y estimada mediante la desviación estándar. En el caso estimado, las rampas extremas tienen valores inferiores a los reales.

En Martín-Martínez et al. (2010b) se propone el uso de los valores parciales de la curva de distribución acumulada de las rampas, denominados como PRR (porcentaje de rampas), como valores más conservadores que la desviación estándar para la caracterización de las mismas. Las rampas extremas positivas vienen definidas por el valor PPRR-1, que representa el valor la rampa que solo se supera con una probabilidad del 1\%, y las rampas negativas por PNRR-99, representa a su vez el valor de rampa a la que solo es inferior un $1 \%$ de las mismas.

\subsubsection{Fluctuaciones según rangos de potencia}

Tal y como se ha descrito anteriormente la potencia eólica obtenida a partir de la velocidad del viento viene dada por la curva de potencia del aerogenerador en cuestión. Existen diferentes tipos de curvas de potencia 


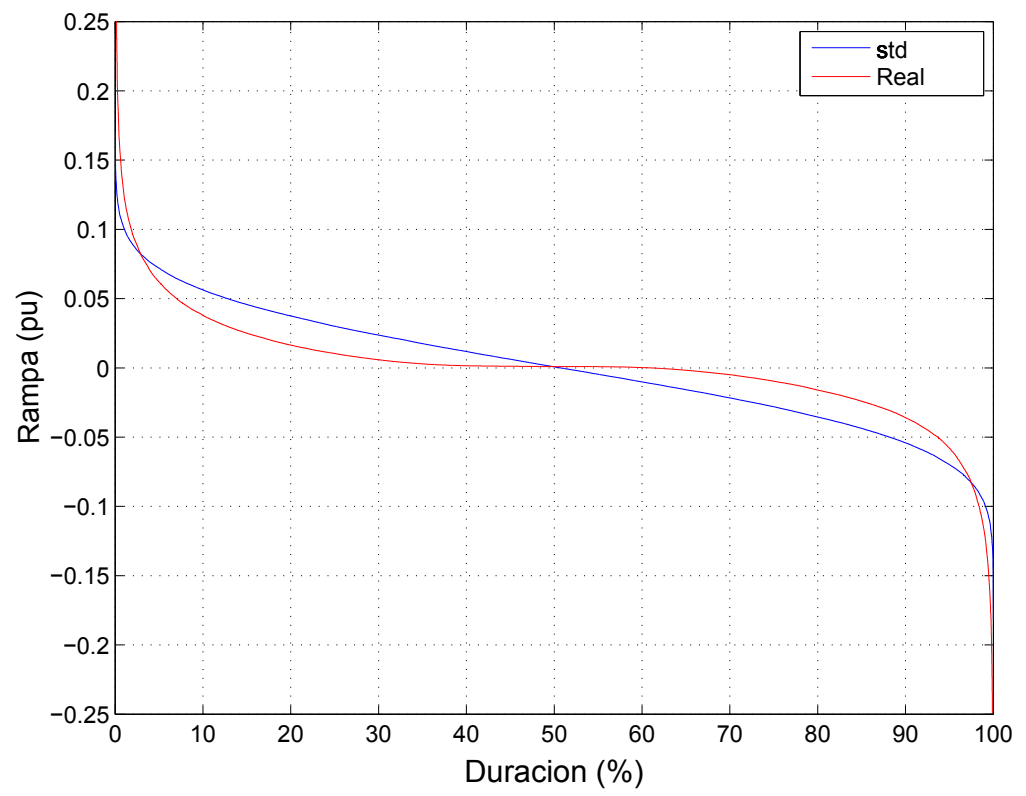

Figura 2.11: Comparación entre una distribución acumulada de rampas reales y un ajuste mediante una distribución normal a partir de su desviación estándar

según el sistema de control y accionamiento del ángulo de incidencia (pitch). Los diferentes tipos de curva se muestran en la figura 2.12.

En general, las características de la curva de potencia de un aerogenerador hacen que dentro de la zona de producción existan dos áreas diferenciadas una zona a carga parcial y otra área a carga nominal, tal y como se observa en la figura 2.7. En la zona de carga parcial se pueden dar tanto rampas positivas como negativas y están limitadas, las rampas negativas por la velocidad de arranque, ya que por debajo no hay producción, y las rampas positivas por el cambio a carga nominal. En la zona a carga nominal no puede producirse rampas positivas debido a la limitación de potencia impuesta mediante la modificación del ángulo de incidencia del viento sobre las palas, por el contrario, en esta zona el margen para rampa negativa es el mayor que puede sufrir el aerogenerador en el caso que se supere la velocidad de corte (20-25 $m / s)$.

En el caso de parques eólicos la producción conjunta de los distintos aerogeneradores presenta ciertas diferencias a la descrita para un aerogenerador. Existen casos en los que los aerogeneradores funcionan en distintos regímenes, como por ejemplo, momentos en los que ciertos aerogeneradores están funcionando a carga parcial mientras que otros ya han alcanzado la carga nominal. Para analizar las fluctuaciones de parques eólicos según el rango de potencia en el que están funcionando se han calculado los valores de PNRR-99 de rampa negativa para diferentes rangos de potencia con los 
CAPÍtulo 2. Variabilidad y predictibilidad de la energía eólica y sus efectos sobre la red eléctrica

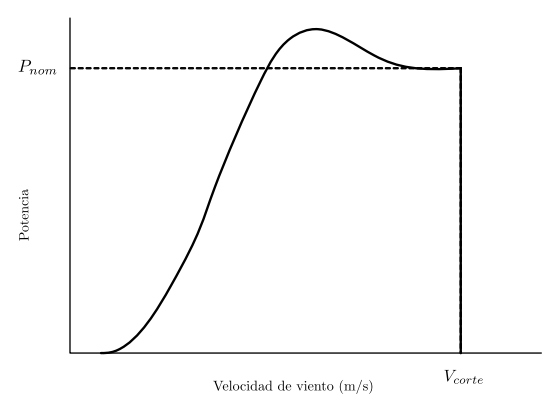

(a) Control aerodinámico pasivo

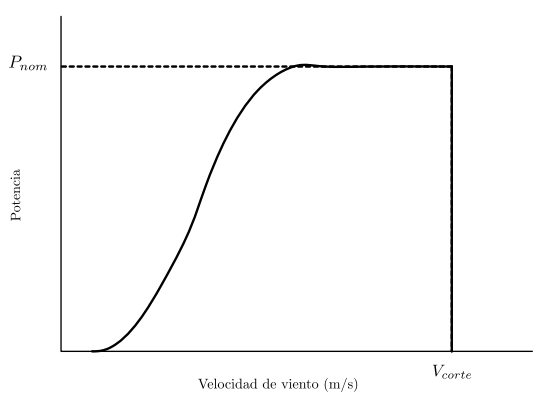

(b) Control aerodinámico activo

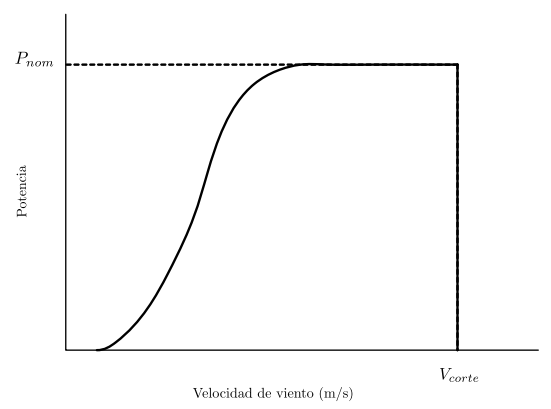

(c) Control ángulo de incidencia

Figura 2.12: Tipos de curva de potencia según el tipo de sistema de control y accionamiento del ángulo de incidencia

parques objeto de estudio descritos en 3. Los valores de PNRR-99 de rampa negativa se muestran para rangos de potencia con una resolución de 0.1 p.u. Para obtener estos valores se han ordenado los valores de rampa para cada nivel de potencia de forma que se obtenga el valor de PNRR-99 para cada rango de potencia.

De forma general, los valores de PNRR-99 de rampas negativa aumentan hasta que se alcanza un rango aproximado de 0.55 p.u., a partir de ahí, los valores se incrementan en todos los casos menos en los parque WF4, WF7 y WF13. Estos tres parques tienen en común que están constituidos por aerogeneradores con control stall pasivo. El caso de WF2 es similar ya que también está constituido por un $41 \%$ de aerogeneradores de ese tipo. Este comportamiento es detallado en Martín-Martínez et al. (2010b). Lo descrito se puede apreciar en las figuras 2.13 y 2.14.

En la figura 2.13, se presentan valores de PNRR-99 de rampa negativa según el rango de potencia en el que se produzcan para los parques con una potencia nominal por debajo de $30 \mathrm{MW}$, como son WF3, WF5, WF9, WF10, WF12, WF16 y WF17. Se aprecia que los valores más negativos se producen normalmente en el área de carga parcial alta (0.65-0.85 p.u.).

En el caso de los parques con mayor potencia instalada, por encima de 


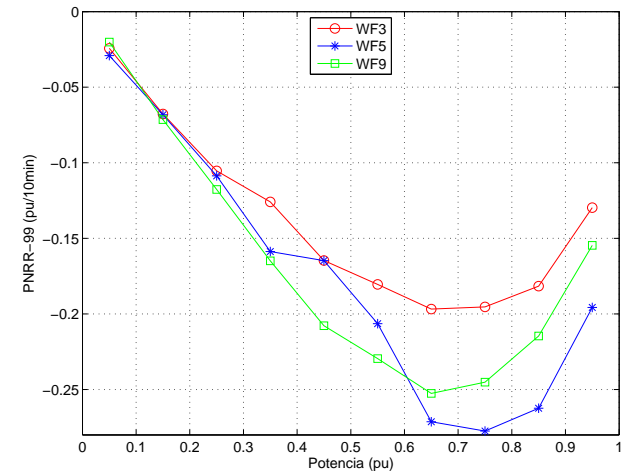

(a) 2007 data WFs.

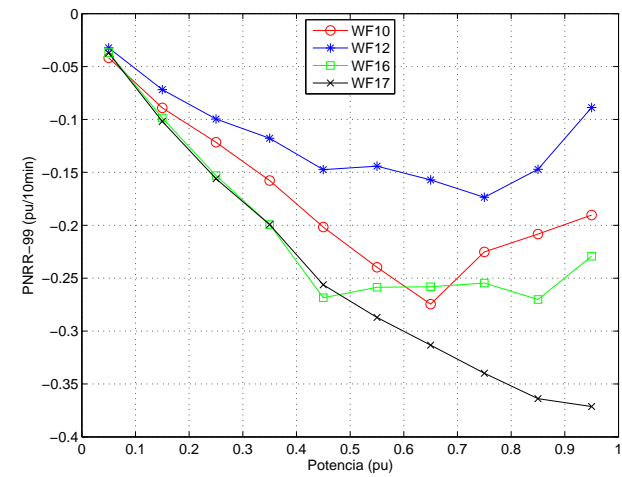

(b) 2008 data WFs.

Figura 2.13: $99 \%$ rampa negativa según rango de potencia para parques $P<30 M W$

30 MW, como son WF1, WF2, WF4, WF6, WF7, WF8, WF11, WF13, WF14 y WF15, los valores de PNRR-99 de rampas negativas aparecen en un rango más amplio a carga parcial (0.5-0.85 p.u.), tal y como se muestra en la figura 2.14. Esto se debe a que en rangos superiores la mayoría de los aerogeneradores están en la zona plana correspondiente a la potencia nominal y por lo tanto la variabilidad es menor. Los resultados obtenidos son similares a los conseguidos por Sørensen et al. (2007) para un gran parque eólico marino como es Horns Rev.

En cuanto a la potencia agregada de todos los parques, tal y como se muestra en la figura 2.15 y teniendo en cuenta que la máxima potencia obtenida es 0.85 p.u. y solo hay unos pocos datos en el rango 0.8-0.9 p.u., las rampas negativas crecen según aumenta el rango de potencia.

En resumen, se puede que el tipo de curva de potencia y el tamaño del parque eólico influyen en la variabilidad de la potencia generada según el rango de potencia en el que nos encontremos. Así, los parques cuyos aerogeneradores tienen un control stall pasivo presentan mayor variabilidad a cargas parciales altas. Por otra parte, al aumentar el tamaño del parque los rangos con mayor variabilidad se extienden abarcando desde 0.5 p.u. a 0.85 p.u. En el caso de agregaciones, según aumenta el nivel de agregación se tiende a obtener un variabilidad que va en aumento con el aumento del rango de potencia.

\subsubsection{Efecto de suavizado (Smoothing effect)}

El efecto de suavizado se define como la disminución porcentual de las variaciones eólicas conforme se produce la agregación de unidades de producción eólica. Las fluctuaciones de distintos aerogeneradores se compensan 
CAPÍtulo 2. Variabilidad y predictibilidad de la energía eólica y sus efectos sobre la red eléctrica

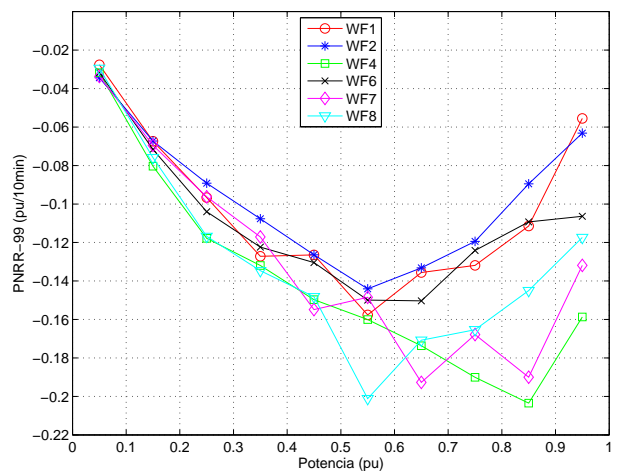

(a) 2007 data WFs.

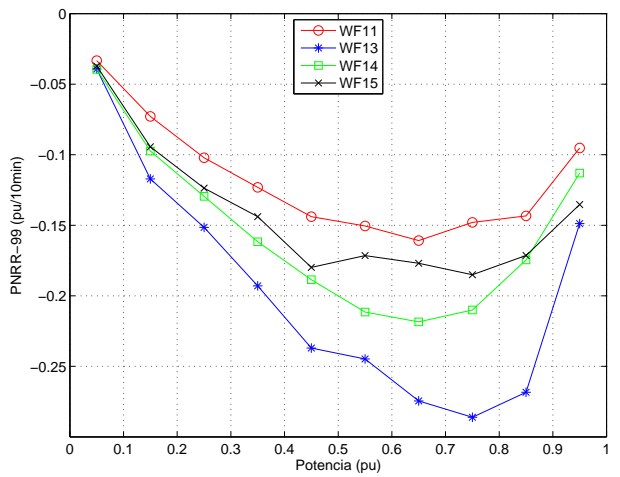

(b) 2008 data WFs.

Figura 2.14: $99 \%$ rampa negativa según rango de potencia para parques $P>30 M W$

entre si cuando obtenemos las variaciones de la producción agregada. La duración de los periodos de calma sin producción disminuye considerablemente al considerar agregaciones en un área más grande, debido a que casi siempre hay producción en alguna unidad de la zona considerada. Del mismo modo, no se alcanzan niveles de producción máximos debido a que no tendremos grandes velocidades de viento en todas las localizaciones de forma simultánea y todos los aerogeneradores no estarán técnicamente disponibles de forma simultánea. Estas características se aprecian en la figura 2.16, donde la potencia generada se "aplana" al incrementar la agregación.

A principios de los años 80 este efecto tenía escasa importancia y se consideraba un problema menor debido a la escasa penetración de la energía eólica en los sistemas eléctricos. Para caracterizar la agregación se utilizaba una expresión teórica obtenido por Lipman et al. (1980) que suponía un comportamiento ideal de un grupo infinito de aerogeneradores impulsados por una serie totalmente correlacionada en el tiempo de velocidad de viento. Este llamado efecto de suavizado de las fluctuaciones rápidas de un conjunto de aerogeneradores se observaba más notorio para un gran número de aerogeneradores que comparten una conexión eléctrica común, ya que esa situación representa la condición ideal de enfoque de Lipman que permiten definir el efecto de suavizado como:

$$
\sigma(P W P)=\frac{\sigma(P W T)}{\sqrt{N}}
$$

donde $\sigma(P W P)$ representa la desviación estándar de la producción en el punto de conexión y $\sigma(P W T)$ es la desviación estándar para un solo aerogenerador. El factor proporcional $\sqrt{N}$, es la raíz cuadrada del número de aerogeneradores N. A partir del trabajo de Lipman, en Beyer et al. (1990b,a) 


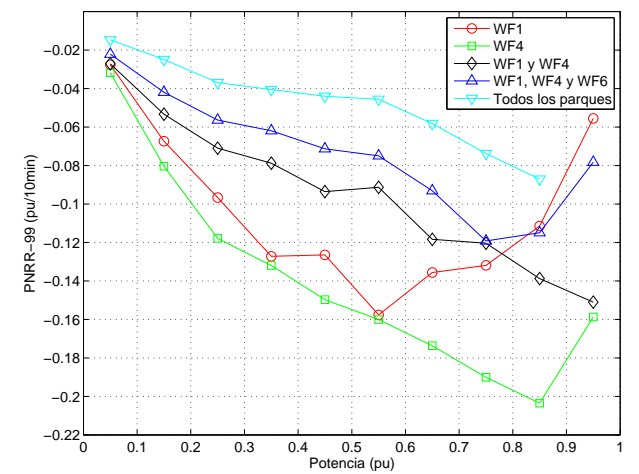

(a) 2007 data WFs.

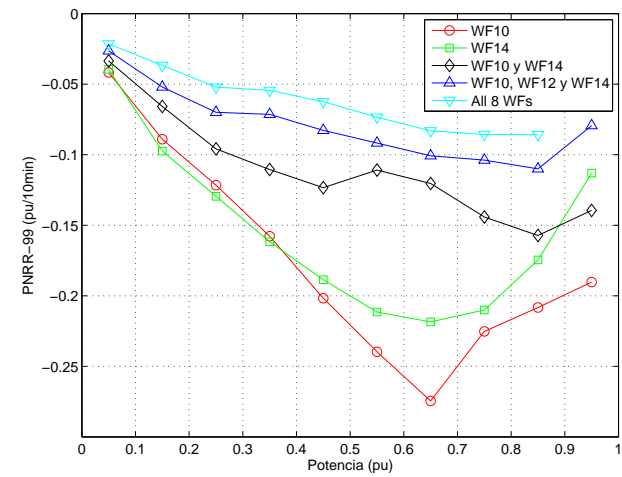

(b) 2008 data WFs.

Figura 2.15: $99 \%$ rampa negativa según rango de potencia para producción agregada

se confirma que este efecto se produce también en el dominio de la frecuencia y se define $1 / \sqrt{ }(N)$ como una regla para la cancelación de altas frecuencias de las fluctuaciones. Esta relación sigue considerándose como referencia en caso de que no se dispongan de datos experimentales para realizar pruebas estadísticas representativas.

El efecto de suavizado también se puede observar en el rango de producción, máximo y mínimo obtenido en un periodo considerablemente largo. Por esta razón se propone como parámetro para medir el efecto de suavizado a la anchura de fluctuación. La anchura de fluctuación $\left(F_{w}\right)$ es un parámetro utilizado para caracterizar el efecto de suavizado por Asari et al. (2002). Para un intervalo dado $(0, T)$, este parámetro se define como:

$$
F_{w}(T, N)=P_{\max }^{[0, T]}-P_{\min }^{[0, T]}
$$

Donde $P_{\max }^{[0, T]}$ y $P_{\min }^{[0, T]}$ son respectivamente la potencia máxima y mínima en el intervalo $[0, T]$ generada por un número de aerogeneradores $N$. Para un cierto periodo y un numero de aerogeneradores, $F_{w}$ es una función cuya PDF depende de las características estocásticas del viento. En un periodo dado, el valor esperado de esta función $\left(E\left[F_{w}\right]\right)$ es, a su vez, una función decreciente del número de aerogeneradores $N$, ya que el efecto de suavizado es más pronunciado según aumenta el número de aerogeneradores considerados. Por el contrario si se va aumentando el periodo T, el valor de $F_{w}$ se verá incrementado tal y como se deduce de su definición 2.44. Además del máximo y mínimo de producción se puede representar toda producción ordenada mediante una curva de duración. Cuanto más plana sea esta curva mayor será el efecto de suavizado presente.

En un parque eólico, este efecto depende de la topografía local y de las condiciones meteorológicas. Además, en este caso la agregación solo afecta a 
Capítulo 2. Variabilidad y predictibilidad de la energía eólica y sus efectos sobre la red eléctrica

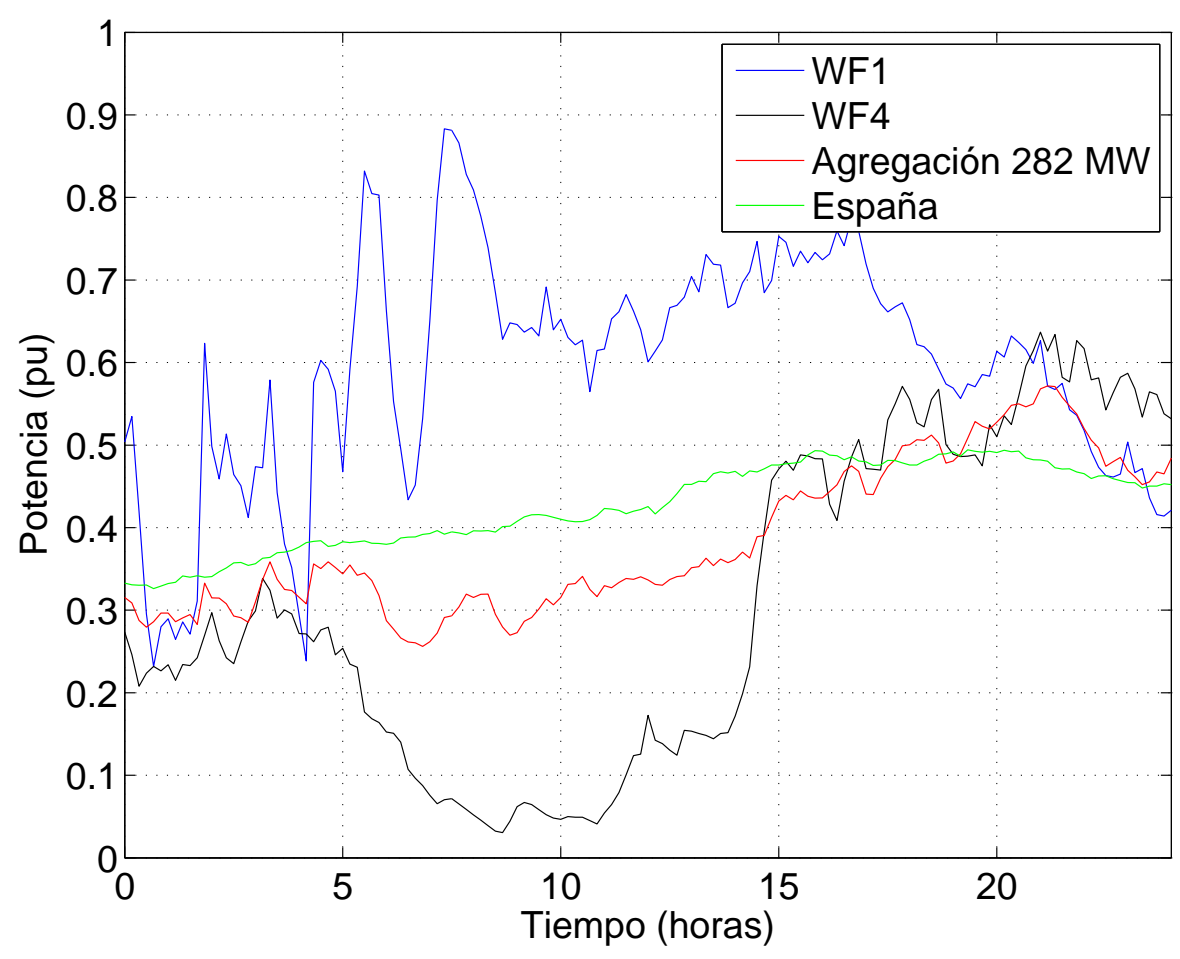

Figura 2.16: Comparación entre la producción de distintas agregaciones eólicas

las fluctuaciones de alta frecuencia. El efecto de suavizado viene dominado por la correlación espacial del viento por lo que a nivel de parque es de vital importancia estudiar la coherencia del viento para establecer los parámetros de suavizado según Nanahara et al. (2004a) y Nanahara et al. (2004b).

En una región amplia, la diferencias climatológicas entre diversas áreas con parques eólicas instalados tienen un gran peso en el nivel de suavizado presente en la producción agregada, Holttinen (2004).

El efecto de suavizado también puede ser observado a partir de los valores definidos en el apartado anterior para medir las rampas, como son la desviación estándar y los PRR. Así, a medida que el nivel de agregación aumenta estos parámetros se reducen y para niveles altos tienden a converger. Esta es una consecuencia del teorema del límite central que indica que la suma de variables independientes se aproxima a una distribución normal. Si consideramos parques muy distantes entre sí, se puede considerar que sus valores de potencia generada no están correlacionados y son independientes. 


\subsubsection{Eventos extremos. Causas y clasificación}

Existen dos parámetros que caracterizan los cambios en la producción eólica, el tamaño de rampa, definido como el valor del cambio de la producción que ocurre como un porcentaje de la potencia instalada del parque eólico o agregación, y la duración, definida como la cantidad de tiempo en la que el cambio ocurre. De esta forma, según se tengan mayores tamaños de rampa y duraciones más cortas, más extremos serán los eventos. Un evento se puede definir como una variación de potencia activa de un parque eólico o una agregación que excede un tamaño de rampa mínimo $s_{\min }$, con una duración menor o igual a una duración máxima $d_{\max }$. En la figura 2.17 se muestra la frecuencia de los eventos producidos en el parque WF10 durante el año 2008 según su tamaño de rampa mínimo y su duración máxima. Se aprecia que la frecuencia de los eventos decrece exponencialmente según se incrementa el tamaño de rampa mínimo y también decrece al tiempo que disminuye la duración, por ejemplo, son menos comunes los eventos con cambios grandes y rápidos.

En estudios como Zack (2007) y Greaves et al. (2009), se definen los eventos extremos o eventos de rampa como aquellos que suponen un cambio del $50 \%$ o más de la potencia instalada con una duración de 4 horas o menos. Una duración máxima de 4 horas asegura que las variaciones son lo suficientemente rápidas y que se incluyen bastantes eventos con un tamaño de rampa mínimo del $50 \%$ para ser identificados en el análisis. En la figura 2.17 se aprecia que los eventos con un cambio del $50 \%$ o más de la potencia instalada y con una duración de 4 horas o menos tienen una frecuencia del $10 \%$ del tiempo.

Tal y como se ha descrito anteriormente las fluctuaciones de potencia eólica se deben fundamentalmente a las variaciones en la velocidad de viento, pero también existen diversos fenómenos técnicos y operacionales intrínsecos de las instalaciones eólicas que hacen que las fluctuaciones de potencia eólica difieran de lo que cabría esperar según las variaciones de viento. Así, hay momentos en los que, debido a la coincidencia en la consecución de varios fenómenos o la acción coordinada de varios de ellos, se producen eventos extremos de fluctuaciones de potencia eólica. Estos eventos extremos corresponden, normalmente, con tamaños grandes y con duraciones pequeñas, siendo su frecuencia relativamente reducida. Las técnicas de predicción avanzadas son capaces de aliviar los efectos de estos cambios coordinados en la generación eólica, pero en sistemas con una gran penetración de potencia eólica, estos eventos extremos aumentan los costes de operación y pueden afectar a la seguridad de dichos sistemas.

Los eventos extremos se pueden clasificar en base a su causa o causas asociadas. Tal y como se describe en Martín-Martínez et al. (2012), las causas principales son las siguientes: 
CAPítulo 2. Variabilidad y predictibilidad de la energía eólica y sus efectos sobre la red eléctrica

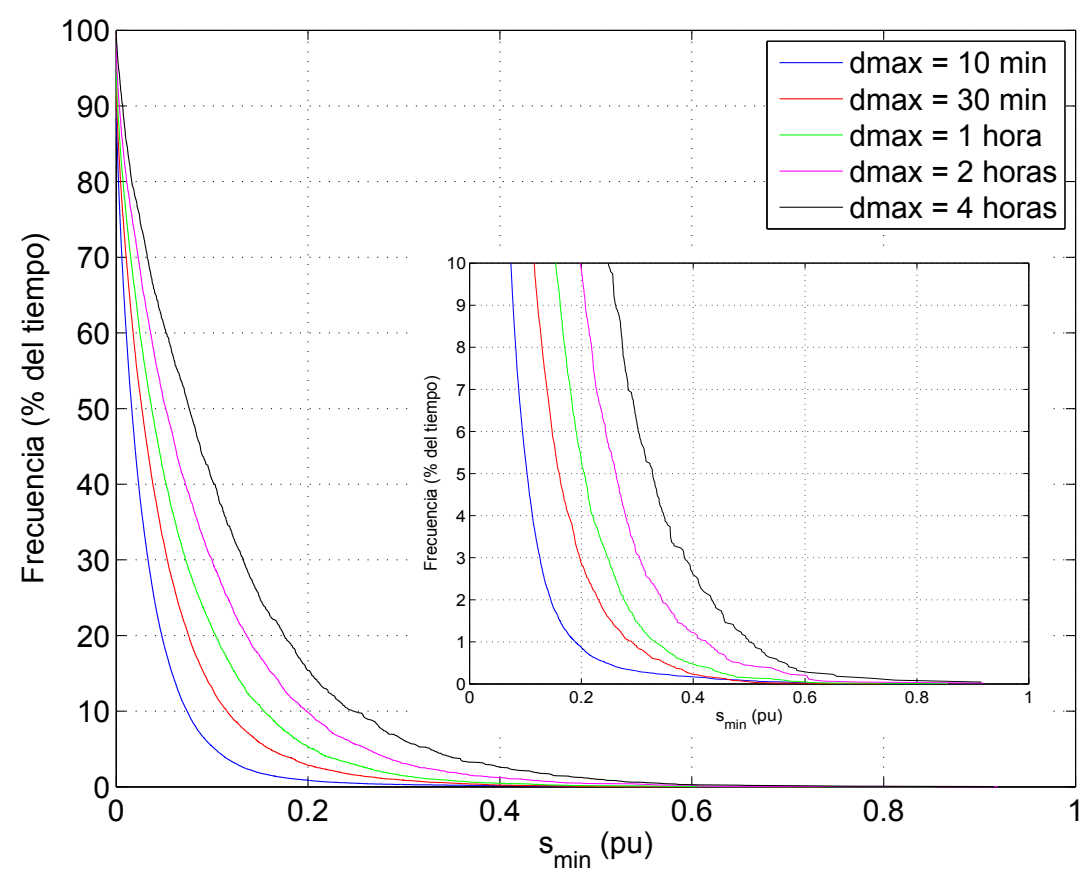

Figura 2.17: Frecuencia de los eventos según su tamaño mínimo $s_{\min }$ y su duración máxima $d_{\max }$ para el parque WF10

- Fenómenos meteorológicos. Los eventos causados por los fenómenos meteorológicos tienen normalmente un patrón de una subida rápida en la velocidad de viento seguida de una caída más o menos rápida. En áreas reducidas, estos eventos pueden provocar ráfagas persistentes aumentando la variabilidad de la velocidad de viento. En el Sistema Eléctrico Español, las rampas negativas provocadas por estos fenómenos, ya sea por superar la velocidad de corte o por una bajada de velocidad siguiente al fenómeno, pueden afectar seriamente a la seguridad del sistema ya que es necesario un incremento repentino en las centrales de reserva para mantener el balance del sistema.

- Causas técnicas y operacionales. En este grupo las causas principales pueden ser:

- Huecos de tensión. Los huecos de tensión generan una caída repentina en la producción eólica. Esta caída en la potencia generada normalmente se recupera a continuación.

- Velocidad de corte de los aerogeneradores. Cuando la velocidad del viento alcanza la velocidad de corte de un aerogenerador (20$25 \mathrm{~m} / \mathrm{s}$ ), este activa un protocolo de seguridad que incluye su desconexión de la red. Si este fenómeno se extiende por los aerogeneradores presentes en una zona, puede causar un evento afectando 
a la seguridad y la fiabilidad del sistema.

Estos eventos suelen presentar un patrón de una caída pronunciada en la producción eólica seguido por una recuperación hasta aproximadamente la potencia anterior al evento. En otras ocasiones la producción no se recupera.

Adicionalmente todos los eventos se presentan como una combinación de rampas de profundidad y duración variable. Basándose en las características de las rampas, los eventos pueden estar constituidos por uno o varios de los siguientes patrones de rampa, Banakar et al. (2008):

- Caída persistente (die-out). Este patrón consiste en una sucesión de rampas negativas de profundidad considerable. La producción final suele estabilizarse a un nivel bajo. Como ejemplos de eventos que causan este tipo de patrones se encuentran las desconexiones en alta producción por averías, huecos de tensión sin recuperación, vientos muy por encima de la velocidad de corte,...

- Aumento persistente (rise). Este patrón consiste en una sucesión de rampas positivas con una pendiente muy pronunciada. Algunos ejemplos de este tipo de patrón son arranques con alta velocidad de viento, grandes tormentas y frentes de viento.

- Calma momentánea (lull). Se caracteriza por una caída momentánea de la producción seguida de un aumento hasta recuperar la producción. Ambos fenómenos se producen en una rápida sucesión y con rampas muy pronunciadas. Entre los ejemplos que cumplen con estas características están los huecos de tensión con recuperación y vientos en el rango de la velocidad de corte.

- Ráfaga (gust). Consiste en un aumento pronunciado seguido de una caída hasta aproximadamente la producción previa. Este patrón es el contrario a la calma momentánea. Se consideran ejemplos de este tipo de patrón pequeñas tormentas y vientos racheados.

\subsection{Predicción de la energía eólica}

Con la metodología actual, los sistemas de predicción de potencia eólica son factibles. Estos mejoran la integración técnica y económica de la energía eólica en el sistema eléctrico. Así, se pueden caracterizar las variaciones de potencia producidas en los parques eólicos por adelantado y se reduce el grado de aleatoriedad o azar atribuido a la energía eólica.

Los diferentes agentes que intervienen en la generación de energía eléctrica a partir de energía eólica se ven beneficiados por las ventajas que supone una predicción con una precisión aceptable. 
CApÍtulo 2. Variabilidad y predictibilidad de la energía eólica y sus 46 efectos sobre la red eléctrica

Para los promotores de los parques eólicos, la importancia reside en conocer la predicción de cada uno de los parques por separado, ya que así pueden hacerse una idea de la energía que van a producir. Así, los promotores de parques eólicos y los agentes del mercado, se benefician de una buena predicción, de forma que su oferta en el mercado eléctrico para un determinado periodo de tiempo sea lo más parecida a la producción real de la cual se va a disponer. Los sistemas de predicción eólica están diseñados para producir una predicción fiable de la potencia que van a generar los distintos parques eólicos. Por otro lado, la posición del Operador del Sistema es muy distinta. Para el es mucho más interesante el conocimiento de la predicción global de la totalidad de los parques instalados, de forma que pueda establecer con antelación la energía eólica global que se inyectará en la red, y así gestionar la potencia que deberán generar las centrales convencionales, con el objetivo de cubrir la demanda total del sistema.

La predicción de energía eólica incrementa el valor económico de este tipo de energía y ayuda a hacer este recurso renovable competitivo con fuentes convencionales. Se reducen los costes de operación en el sistema debido a la menor cantidad de reserva necesaria y se reducen los desvíos de generación que pueden suponer penalizaciones para los agentes.

En contraste con las plantas de potencia convencionales, la producción eléctrica de los parques eólicos depende casi totalmente de las condiciones meteorológicas, particularmente de la magnitud de la velocidad del viento. La generación eólica es no programable, lo cual dificulta su predicción e integración en la red. Sólo se produce energía eólica en determinadas condiciones de viento (de 4 a $25 \mathrm{~m} / \mathrm{s}$ ), y la variabilidad del mismo es impredecible tanto a corto como a largo plazo. Si el viento en un determinado momento es menor del previsto, la potencia generada en los parques eólicos desciende con relación a esa disminución, y esa potencia no generada se debe remplazar con otros tipos de centrales, en forma de reservas a subir, para mantener el balance demanda/generación constante. En otras ocasiones puede suceder lo contrario, que la generación real sea mayor que la prevista, utilizándose las reservas a bajar disponibles y, en caso de superarlas, reduciendo la generación eólica de forma que se elimine el excedente que permita al sistema mantenerse en condiciones de seguridad. Queda clara entonces, la importancia de la predicción de generación eólica, para asegurar un suministro sin comprometer la seguridad y la fiabilidad del sistema eléctrico.

\subsubsection{Errores de predicción}

Los errores de predicción se incrementa a medida que se incrementa el factor de carga debido al aumento de eventos meteorológicos atípicos y a la mayor velocidad media de viento, como se describe en Lang et al. (2002). Estos errores dependen de la localización de referencia del parque eólico, y del comportamiento de los parques en la misma región, así, será mejor que 
la capacidad normalizada de los parques sea similar.

En el caso de la predicción de las rampas de la potencia eólica los errores presentes pueden provenir de 3 fuentes principalmente, error temporal, error de magnitud y error de rampa, tal y como se describe en Potter et al. (2009):

- El error temporal ocurre cuando la predicción realizada es bastante precisa en cuanto a la magnitud pero está temporalmente desfasada con respecto a la predicción real. Este tipo de error puede conllevar un gran error absoluto pese a que la magnitud del evento ha sido caracterizada de forma correcta.

- El error de magnitud consiste en el error total en un instante dado. Se ve acentuado progresivamente cuando existe un error de rampa y se mantiene si el error es temporal.

- El error de rampa consiste en una predicción errónea del ratio de las rampas de la potencia eólica. Al disponer de un rango de cambio distinto se produce un error de magnitud asociado. Este error de magnitud asociado será proporcional a error entre las pendientes de las rampas de la producción real y la predicción.

En la figura 2.18 se muestra una comparación entre predicción y valor real con ejemplos de los errores anteriormente descritos.

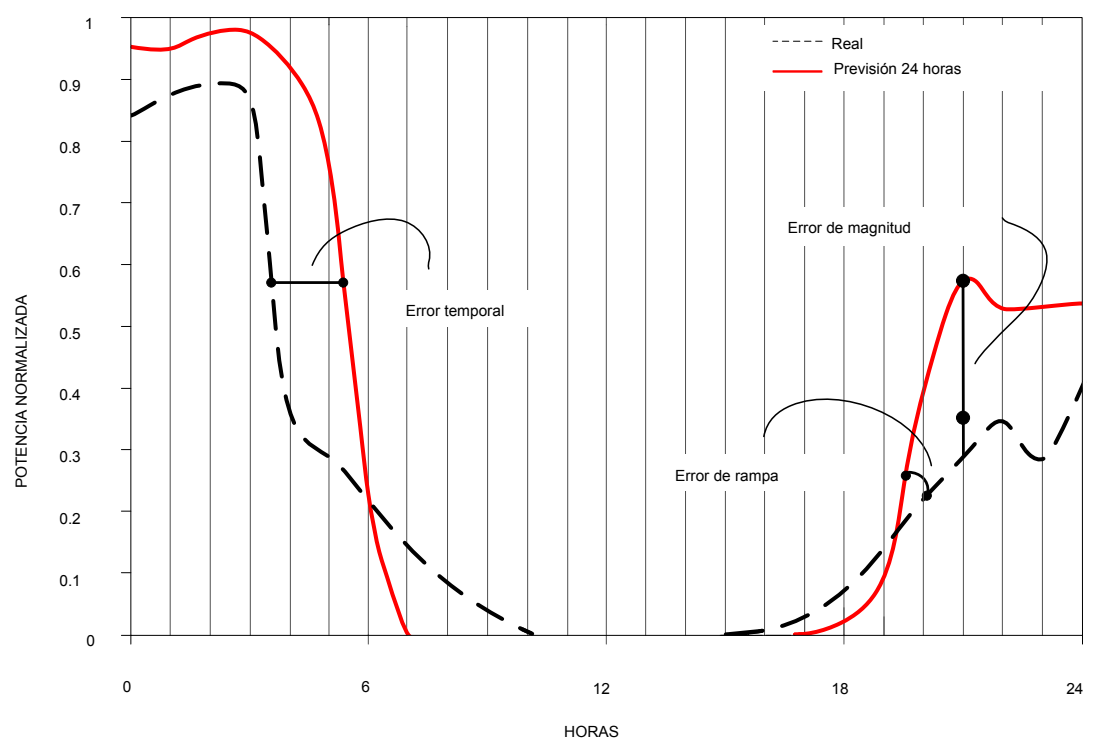

Figura 2.18: Tipos de error de predicción de energía eólica 
CApítulo 2. Variabilidad y predictibilidad de la energía eólica y sus

\subsubsection{Horizontes de predicción}

Para determinar el momento futuro para el cual se realiza la predicción, se define un periodo de tiempo llamado horizonte de predicción, correspondiente a la diferencia temporal entre el instante para el cual se ha realizado la predicción y el instante actual. Así, se pueden clasificar las predicciones en muy corto plazo, corto plazo y largo plazo. El horizonte temporal necesario de las predicciones vendrá dado por el programa de las plantas convencionales y las condiciones lícitas de los mercados eléctricos, que son normalmente de uno o dos días antes. Por tanto, los sistemas de predicción tienen que proporcionar la potencia esperada de salida entre 6 y 48 horas antes. El horizonte de tiempo requerido es muy importante. Desde el punto de vista del modelado hay una gran diferencia entre las llamadas predicciones a corto plazo en escalas temporales de unos cuantos días y las predicciones de muy corto plazo, en un rango de varias horas.

La predicción a muy corto plazo, abarca un horizonte de hasta 4 o 6 horas y tiene como objetivo, en primer lugar, permitir al operador del sistema mejorar la seguridad de la red y la gestión de la regulación secundaria. En segundo lugar, posibilita al propietario de un parque eólico controlar los aerogeneradores y tomar decisiones de seguridad como paradas o arranques de aerogeneradores.

Las predicciones a corto plazo, comprenden horizontes hasta las 48 o 72 horas. Para los propietarios de parques, estas predicciones permiten establecer el plan de mantenimiento a corto plazo de los aerogeneradores y para la estimación de producción de cara al mercado diario. Por otro lado, para el operador del sistema, este tipo de predicciones posibilitan el mantenimiento de las líneas eléctricas y la gestión de congestiones en las mismas

Las previsiones a medio y largo plazo, varían con horizontes entre varios días y meses o años. Las previsiones a varios días tienen aplicación en la predicción de generación de centrales eléctricas convencionales o para programar labores de mantenimiento programadas en los aerogeneradores en días en los que las expectativas de generación sean escasas o nulas.

Mientras que el periodo temporal más largo es más bien descrito por sistemas predictivos climatológicos numéricos, que explícitamente modelan la dinámica de la atmósfera, el rango de muy corto plazo es típicamente dominado por la persistencia de condiciones meteorológicas donde los enfoques puramente estadísticos conducen a mejores resultados predictivos, tal y como se describe en Bossanyi (1985).

El horizonte más utilizado en predicción es el de corto plazo, pues permite tanto la predicción por parte de los productores en los mercados diarios e intradiarios como las necesidades del operador del sistema para su gestión. La resolución habitual de las predicciones de potencia es de una hora, ya que la mayoría de los sistemas y mercados eléctricos se programan siguiendo una escala horaria. 


\subsubsection{Modelos de predicción del viento}

La mayoría de los sistemas predictivos de potencia eólica están basados en los resultados de sistemas numéricos de predicción meteorológica (NWP). Un NWP consiste en el uso de las condiciones atmosféricas como referencia en un modelo matemático de la atmósfera para la predicción de las variables meteorológicas; en el caso de la energía eólica las variables principales son la velocidad y dirección de viento. Por tanto, toda la información sobre los valores futuros, en particular la evolución esperada del vector del viento, proviene de los sistemas NWP.

Como los sistemas NWP extrapolan el estado actual de la atmósfera usando las leyes físicas, la aproximación de las predicciones numéricas, por encima del horizonte de tiempo deseado, es típicamente mucho mejor que cualquier otro tipo de aproximación estadística o climatológica que represente un comportamiento estadístico medio.

\subsubsection{Sistemas numéricos de predicción meteorológica (NWP)}

La física de la atmósfera es bastante compleja, y se puede considerar como un sistema no linear con infinitos grados de libertad. La dinámica se puede describir en términos matemáticos por ecuaciones de movimiento que derivan de los principios de conservación de la masa, los momentos y la transferencia de calor. Pero la estructura no linear de estas ecuaciones fundamentales en general no permiten soluciones analíticas de tal manera que las soluciones aproximadas a los estados reales de la atmósfera se pueden obtener en muchos caos, sólo bajo suposiciones simplificadas. En el contexto de aplicaciones de energía eólica, son importantes dos aproximaciones diferentes para describir la atmósfera. La primera, una previsión del comportamiento dinámico de la atmósfera y, en particular, los campos del vector de viento, dirección y velocidad, se deben obtener resolviendo numéricamente las ecuaciones de movimiento. Estas predicciones numéricas (NWP) proporcionan la entrada para el sistema de predicción de potencia en una resolución espacial bastante amplia de varias decenas de kilómetros. Por tanto, en segundo lugar, se necesitan modelos más simples para describir las condiciones locales de viento en una situación o emplazamiento de interés y así adaptar las previsiones NWP a las condiciones de flujo locales.

Para evitar la dificultad de resolver la gama completa de ecuaciones, se divide la atmósfera en varias capas horizontales para separar los diferentes regímenes de fluidos. Estas capas se definen por los efectos físicos dominantes que influencian la dinámica. Para el campo de la energía eólica, se considera que la troposfera, que abarca los primeros cinco a diez kilómetros por encima de la superficie terrestre, contiene los regímenes de viento que se muestran en la figura 2.19.

Los sistemas NWP simulan el desarrollo de la atmósfera integrando numéri- 
Capítulo 2. Variabilidad y predictibilidad de la energía eólica y sus efectos sobre la red eléctrica

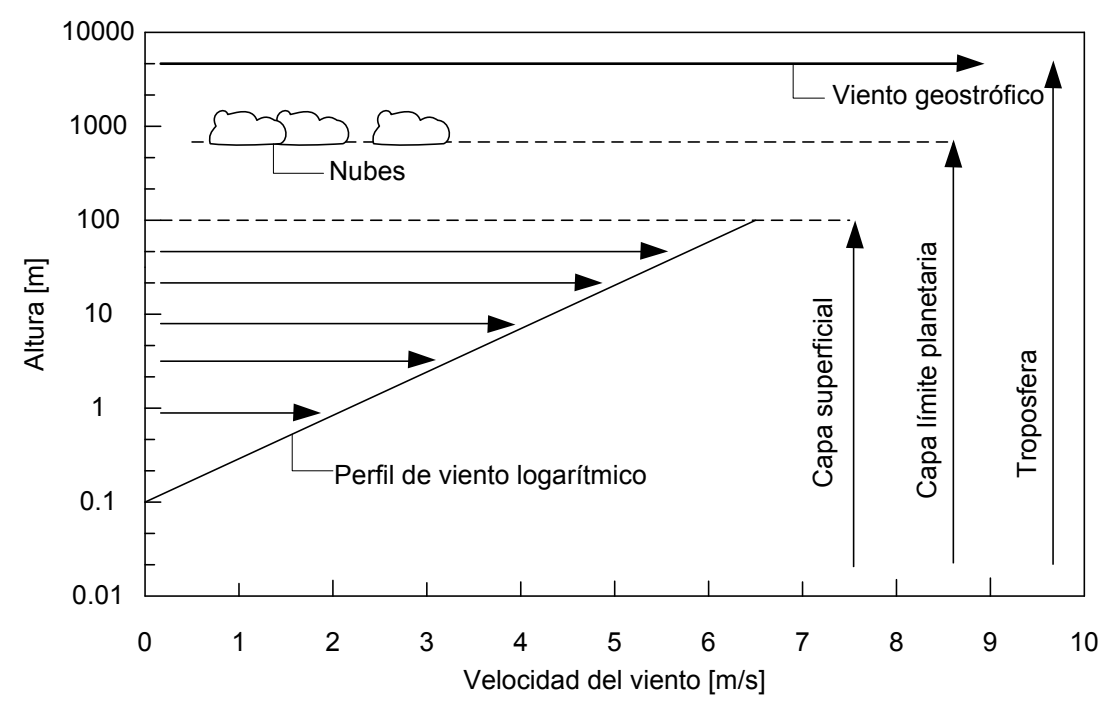

Figura 2.19: Ilustración esquemática de las capas horizontales en la troposfera, que comprende la parte inferior de la atmósfera, importante para la energía eólica. Focken y Lange (2006)

camente las ecuaciones no lineales de los principios de movimiento del estado actual de la atmósfera. Se basan en la resolución de dichas ecuaciones aplicadas a una serie de puntos de una malla o rejilla tridimensional que representa la atmósfera y que, a partir de unos valores iniciales de medidas del estado atmosférico, calculan cómo será la evolución de la misma durante las siguientes horas. Normalmente, con estos sistemas se generan predicciones para las siguientes 48 o 72 horas. Dependiendo de la escala espacial o tamaño de la rejilla, se puede diferenciar entre modelos macro-escala, meso-escala y micro-escala. Así, los modelos NWP explican el comportamiento atmosférico en una larga escala, por ejemplo, los sistemas sinópticos comprenden escalas de longitud desde unos $1000 \mathrm{~km}$ hasta por debajo de $10 \mathrm{~km}$. Las predicciones se hacen por redes que abarcan varios parques eólicos cercanos entre sí, por tanto, el valor previsto puede no ser óptimo para todas las localizaciones dentro de una misma rejilla. Se debe tener precaución si se desean comparar las predicciones con las medidas históricas o las medidas en línea de un punto. Las medidas normalmente tienen que ser medias temporales para poderlas comparar con las predicciones. Ambos tipos de predicciones las suelen realizar los servicios nacionales de meteorología, pues disponen de los datos necesarios. En general, los servicios meteorológicos usan un modelo global con un rango de resolución horizontal desde 100x100 $\mathrm{km}^{2}$ hasta 50x50 $\mathrm{km}^{2}$ para capturar el desarrollo mundial de los sistemas meteorológicos.

En la tabla 2.4 se indican los principales ejemplos de NWP en Europa. Se indica en que países se utilizan y cuál es el horizonte de resolución en 


\begin{tabular}{lll}
\hline Nombre del modelo & Servicio meteorológico & $\begin{array}{l}\text { Horizonte de } \\
\text { resolución } \\
(\mathrm{km})\end{array}$ \\
\hline $\begin{array}{l}\text { Modelo global (GME) } \\
\text { Modelo local (LM) }\end{array}$ & Servicio Alemán de meteorología & 60 \\
ARPEGE & 7 & $19-250$ \\
ALDIN & Servicio meteorológico francés & 9.5 \\
HIRLAM & Suecia, Noruega, España, Dina- & $10-50$ \\
& marca, Finlandia, Islandia, Ir- \\
& landa, Holanda. & \\
ECMWF & $\begin{array}{l}\text { Centro Europeo de predicción de } \\
\text { rango medio }\end{array}$ & \\
& Servicio meteorológico del Reino & $60 / 11$ \\
Modelo Unificado & Unido & \\
&
\end{tabular}

Tabla 2.4: Operación de los sistemas NWP en Europa

kilómetros de cada uno.

Los sistemas NWP no simulan explícitamente la gama completa de los fenómenos atmosféricos que van desde grandes tormentas recogidas por los sistemas meteorológicos de gran escala, a la disipación de energía mediante turbulencia de micro-escala. Modelar todos los efectos consumiría demasiado tiempo de cálculo y produciría más detalles de los necesarios para una aplicación específica. Debido a esto, los modelos numéricos que describen las dinámicas de la atmósfera, típicamente se centran en efectos concretos y pueden, por tanto, clasificarse de acuerdo a su escala espacial o temporal. Esta clasificación se aprecia en la figura 2.20, donde se muestran las escalas longitudinales y temporales de los fenómenos atmosféricos típicos. Es importante aclarar que los modelos numéricos no sólo difieren en su resolución espacial, sino también en el establecimiento de las ecuaciones de movimiento, adaptadas al tipo de efectos físicos que se tienen que describir en una escala específica.

Básicamente, los modelos matemáticos se dividen en tres clases:

- Modelos NWP: los cuales abarcan desde $1000 \mathrm{~km}$ hasta $10 \mathrm{~km}$.

- Modelos meso-escala: los cuales abarcan desde $10 \mathrm{~km}$ hasta $1 \mathrm{~km}$.

- Modelos micro-escala: los cuales abarcan desde varios cientos de km hasta $0.01 \mathrm{~m}$.

Los modelos meso-escala se guían por modelos NWP de gran escala, como 
CAPítulo 2. Variabilidad y predictibilidad de la energía eólica y sus 52 efectos sobre la red eléctrica

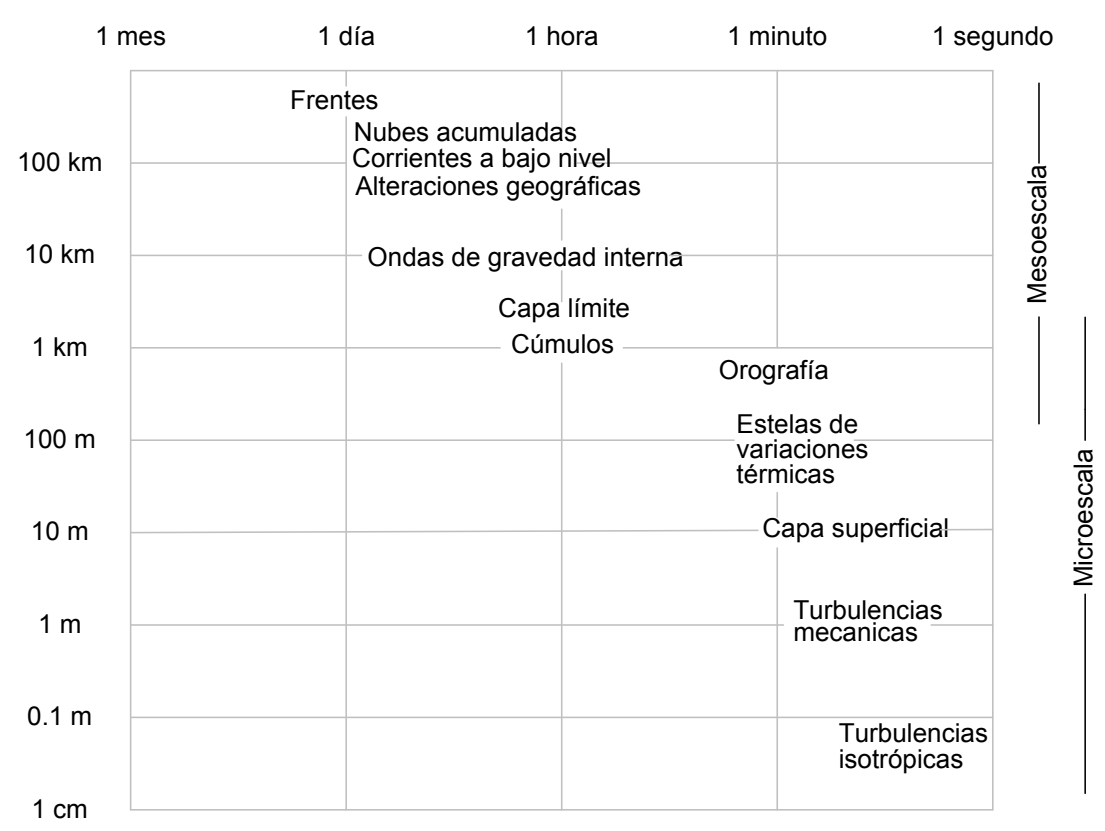

Figura 2.20: Órdenes de magnitud típicos temporales y espaciales de los fenómenos atmosféricos. Focken y Lange (2006).

por ejemplo, los modelos NWP que proporcionan las condiciones límite. Este proceso se llama anidación, donde la idea reside en usar un modelo de alta resolución solo en áreas de especial interés para cubrir los efectos atmosféricos en una escala menor, que no pueden resolverse por los modelos ordinarios. En contraste con los modelos NWP macro-escala, los modelos meso-escala son típicamente no hidrostáticos, lo que significa que pueden modelar directamente los movimientos verticales, como por ejemplo, los debidos a la flotabilidad. En particular, la ventaja de los modelos meso-escala reside en una consideración de los efectos térmicos más detallada, muy beneficioso en terrenos con gran radiación solar y cercanos a la costa para capturar las brisas marinas. Estos modelos también son importantes en terrenos complejos como en España o Noruega, donde la velocidad y la dirección del viento no son homogéneas.

Para explicar los efectos de flujo en la proximidad de un parque eólico, se usan modelos micro-escala con diferentes grados de complejidad. La clase más conocida de modelos de masa constante únicamente se basa en el principio de conservación de la masa. Por tanto, los efectos fraccionales y térmicos son normalmente ignorados o añadidos posteriormente como corrección. Los modelos de masa constante son capaces de modelar el efecto de terrenos ligeramente complejos pero tienden a sobrestimar los gradientes de velocidad del viento horizontal si el terreno tiene mucha pendiente. Las soluciones de 
flujo tienen típicamente ciertas simetrías de tal manera que se pueden calcular muy eficientemente. Como el campo de flujo inicial tiene gran impacto en los resultados, la influencia de la rugosidad dependiente de la dirección puede implementarse fácilmente.

\subsubsection{Modelos de predicción de potencia eólica}

Existen dos aproximaciones básicas para la predicción de la energía eólica a corto plazo, los modelos físicos y los modelos estadísticos.

- Los modelos físicos consideran las propiedades físicas concretas del lugar para adaptar las predicciones de viento a cada emplazamiento o parque. Para esta adaptación se utilizan submodelos de meso-escala o de micro-escala que, partiendo de las condiciones iniciales y de contorno obtenidas de un modelo atmosférico NWP de mayor escala. Estos submodelos calculan la velocidad del viento incidente en los aerogeneradores del parque, adaptándola a la altura del buje para el emplazamiento considerado, para posteriormente calcular la predicción de potencia por medio de la curva de potencia.

- Los modelos estadísticos consisten en una emulación entre las predicciones meteorológicas, las medidas históricas y la potencia generada, a partir de métodos estadísticos con parámetros estimados, sin tener en cuenta ningún fenómeno físico. De entre los modelos estadísticos se puede encontrar la familia de las series temporales, que solo utilizan valores pasados de las variables como datos de entrada del modelo. También existen otros modelos mixtos en los que, además de valores pasados, se utilizan como entradas los valores de predicción meteorológica de modelos atmosféricos, relacionándolos con los valores de potencia histórica u otros valores históricos medidos.

El modelo final de predicción suele ser una combinación de modelos físicos y estadísticos. El modelo físico estima con detalle el viento en el parque eólico y el estadístico utiliza la información física y las medidas de potencia almacenadas de ese emplazamiento para minimizar el error.

En cuanto a la metodología de los modelos se pueden distinguir dos grupos. En el primer grupo se sitúan los modelos en los que se realiza una predicción de la velocidad de viento y, posteriormente se realiza la conversión de velocidad de viento a potencia mediante el uso de una curva de potencia, ya sea empírica o establecida por el fabricante del aerogenerador. En el otro grupo están los métodos que realizan una predicción directa de la potencia generada sin el uso de un paso previo en el cual se realiza una predicción de la velocidad de viento.

En la tabla 2.5 se muestra una relación de métodos, tanto para la predicción de velocidad de viento como para la predicción directa de potencia 
CAPítulo 2. Variabilidad y predictibilidad de la energía eólica y sus efectos sobre la red eléctrica

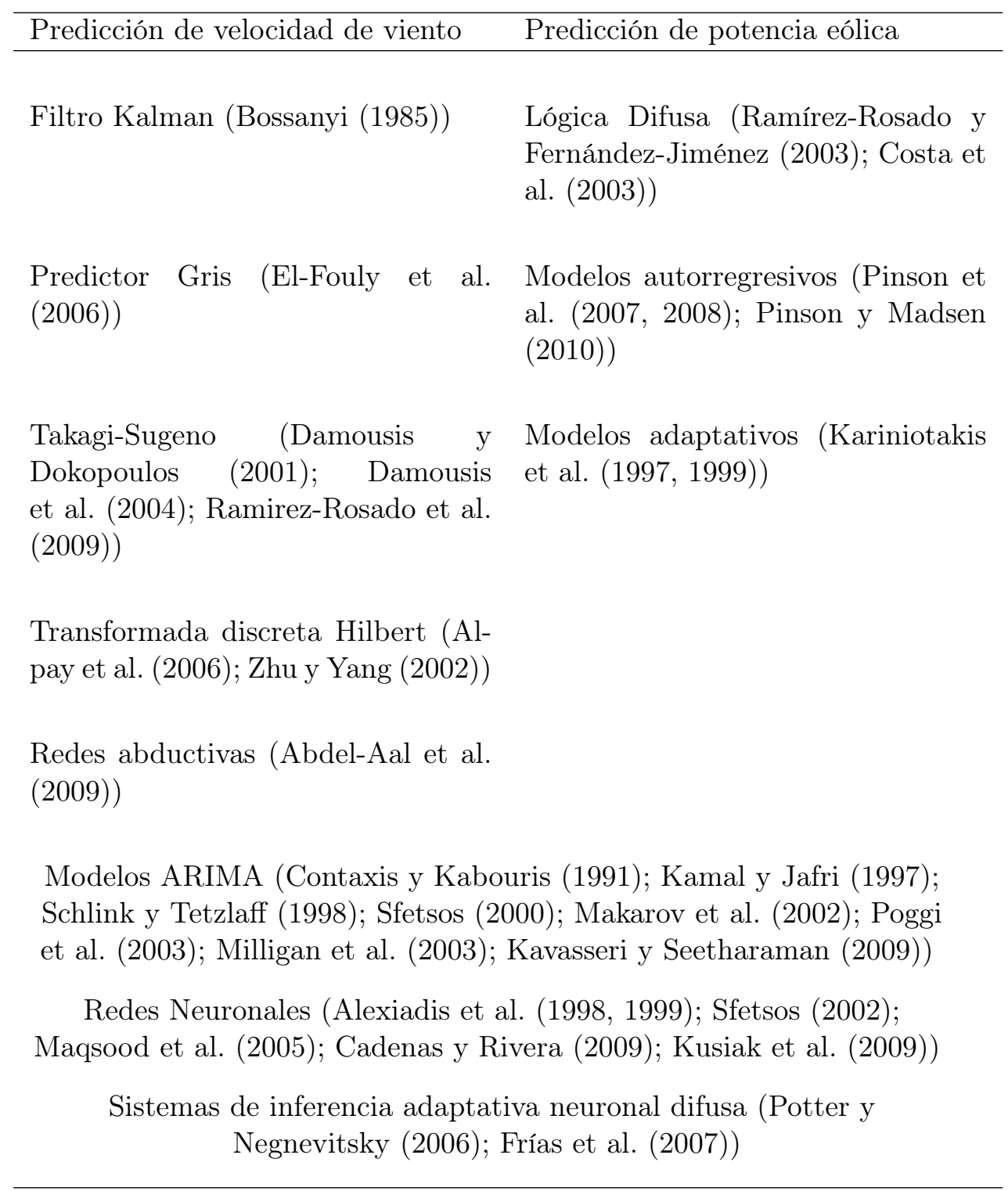

Tabla 2.5: Métodos usados para modelos de predicción de velocidad de viento y de potencia eólica. Monteiro et al. (2009) 
eólica.

\subsubsection{Predicción regional de potencia eólica}

En el caso del operador del sistema, el interés de la predicción de potencia eólica radica en el total de la agregación de todos los parques de la región de modo que se puedan establecer la programación de las reservas del sistema. En estos tipos de modelos de predicción el error suele reducirse debido a la agregación espacial y temporal de todos los parques de la región. En este caso el modelo consiste en extrapolar la potencia total generada de las predicciones aportadas por un número representativo de parques eólicos, para los cuales se han aportado los datos necesarios mediante un sistema de predicción. Los métodos de predicción regional mas utilizados pueden clasificarse según:

- Directa. Se relaciona directamente la generación y los datos NWP de uno o mas parques eólicos a la predicción regional

- En cascada. Este método se divide en dos etapas: primero se lleva a cabo la predicción de los parques de referencia y después la suma de la predicciones se extrapola a la generación global de la región.

- Por subregiones. El proceso de este método esta constituido por 3 pasos: los parques eólicos se agrupan, se desarrolla un modelo para cada grupo resultante y finalmente de la agregación de la predicción de todos los grupos resulta la generación total de la región.

- Combinada. Utiliza combinaciones de los métodos anteriores.

Siebert (2008) afirma que no existe una diferencia notable entre estos tipos de modelo. Del mismo modo establece que la relación entre la generación de un solo parque eólico y la generación regional es fuertemente lineal.

La magnitud del error en la predicción regional depende fuertemente del tamaño de la región, a mayor región, menor es el error resultante, y así se describe en Focken et al. (2002). Cuando agregamos una cierta cantidad de parques eólicos de una zona relativamente extensa, los errores no correlacionados se cancelan debido al efecto de suavizado existente, tal y como se describe en Focken et al. (2001b). Adicionalmente, Siebert et al. (2006) muestra que al incrementar el número de parques eólicos, disminuye el error inicialmente. Aunque después de alcanzar un número específico de parques eólicos el error vuelve a incrementarse. Para ampliar este fenómeno, en Gastón et al. (2006) se identifica que existe un límite en la reducción del error debido a la agregación de parques, de hecho, la agrupación de más de 3 parques eólicos no necesariamente resulta con la reducción del error. Esto hace que sea de suma importancia la selección de los parques de referencia para cada región definida. 
CApítulo 2. Variabilidad y predictibilidad de la energía eólica y sus 56 efectos sobre la red eléctrica

Por otra parte, Pinson et al. (2003) concluye que en modelos avanzados y para horizontes de tiempo de 15 horas, el efecto de suavizado tiene una mayor influencia que la persistencia. La persistencia es un método simple según el cual se considera que la producción eólica en el futuro será la misma que la producción en la actualidad. Este método se usa típicamente para evaluar el comportamiento de modelos de predicción avanzados. En cambio para horizontes entre 1 y 5 horas, la persistencia es el efecto con mayor presencia.

\subsubsection{Ejemplos de sistemas de predicción de potencia eólica}

En este apartado se detallan dos de los modelos más conocidos y utilizados, como son el Previento y el Sipreólico. Se indican sus principales características, los submodelos por los que están compuestos y los sistemas en los que se utilizan.

\subsubsection{Previento}

El sistema Previento ha sido desarrollado por la Universidad de Oldenburgo. Típicamente, la predicción numérica de la velocidad del viento es el resultado los sistemas NWP con una cierta resolución espacial. En el caso particular de Lokalmodell, utilizado por el Servicio Meteorológico Alemán, la resolución espacial es de $7 \times 7 \mathrm{~km}$. Así, la salida de NWP tiene que ser modificada con las condiciones locales de cada lugar específico, en particular considerando la orografía y la dirección del viento dependiente de la rugosidad superficial. Para calcular la velocidad del viento a la altura del buje es necesario modelar en detalle la estratificación térmica de la atmósfera. Seguidamente, la velocidad del viento se transforma en potencia de salida por medio de la curva de potencia. Las curvas de potencia utilizadas deben ser obtenidas a partir de las características de las condiciones meteorológicas del emplazamiento para las que han sido designadas. En un parque eólico, los efectos de estela entre las turbinas pueden llevar a una disminución de más de un $20 \%$ en la potencia eléctrica generada, por lo tanto, estos efectos también deben ser considerados. Como resultado, Previento proporciona la potencia de salida prevista de un solo parque eólico. Si los datos medidos de viento son factibles, se aplica una corrección estadística de los errores sistemáticos de previsión, usando técnicas de regresión linear. La figura 2.21 muestra el esquema básico del sistema de predicción Previento.

En la práctica, las industrias energéticas y los operadores del sistema, requieren la potencia de salida combinada de muchos parques eólicos dispersos espacialmente en una región, en vez de la potencia de un solo parque. Consecuentemente, Previento contiene un avanzado algoritmo de reescalado que determina la potencia de salida esperada de todos los parques eólicos en un área concreta, basándose en un número determinado de lugares represen- 
tativos, seleccionados de manera apropiada, y teniendo en cuanta los efectos de suavizado.

Los módulos más importantes de Previento son:

- Rugosidad superficial detallada.

- Estratificación térmica.

- Orografía del terreno.

- Efectos que se producen en los parques eólicos.

Previento proporciona una predicción de la potencia eléctrica esperada para un horizonte temporal superior a 48 horas. Así mismo, modela la capa límite de acuerdo a la rugosidad, la orografía y los efectos de los obstáculos. Para el cálculo de la velocidad del viento a la altura del buje es importante la variación diaria de la estratificación térmica de la atmósfera, que suele cambiar el perfil logarítmico. La potencia eléctrica esperada de cada lugar se calcula usando las características de potencia específicas de las turbinas. Debido a la necesidad de la potencia eléctrica conjunta de los parques eólicos de una región, se desarrolló un método de reescalado para prever la potencia esperada de una región entera.

El problema de generar una predicción regional es que no es posible predecir la potencia eléctrica de cada turbina por separado. Se divide la región en sub-regiones y para cada sub-región se determinan lugares representativos. Después, la previsión de estas zonas representativas se reescala para conseguir la potencia de las sub-regiones. En este proceso se consideran dos aspectos: el efecto de suavizado presente y las características estadísticas de las variables. Debido a los efectos espaciales de suavizado, el error de predicción para la potencia combinada se reducirá en comparación con el de un lugar aislado. No sólo el error de predicción es una medida de la calidad de la predicción, sino también las características estadísticas, como por ejemplo, la fluctuación de la potencia eléctrica esperada. Las fluctuaciones rápidas de la potencia eléctrica esperada de un solo lugar se traspasan a la previsión regional mediante un reescalado linear. El gradiente de potencia para la previsión regional es menor que para lugares aislados. El alcance de los efectos de suavizado depende principalmente de la correlación espacial de la potencia de salida de los lugares representativos, del número de los mismos y de la distribución de éstos, tal y como se describe en Focken et al. (2001a).

Para que el reescalado lineal sea posible, deben cumplirse los siguientes criterios:

- Las fluctuaciones de las medidas regionales y la potencia prevista tienen la misma magnitud.

- La magnitud de las fluctuaciones de la potencia regional converge cuando incrementa el número de turbinas. 
CAPítulo 2. Variabilidad y predictibilidad de la energía eólica y sus efectos sobre la red eléctrica

La primera característica importante es que la potencia prevista muestre las mismas características que la potencia medida, para reducir las fluctuaciones del conjunto de los diferentes lugares. Esto significa que la previsión de la potencia esperada de salida de las regiones tiene las mismas fluctuaciones que en la realidad (comparado con las medidas).

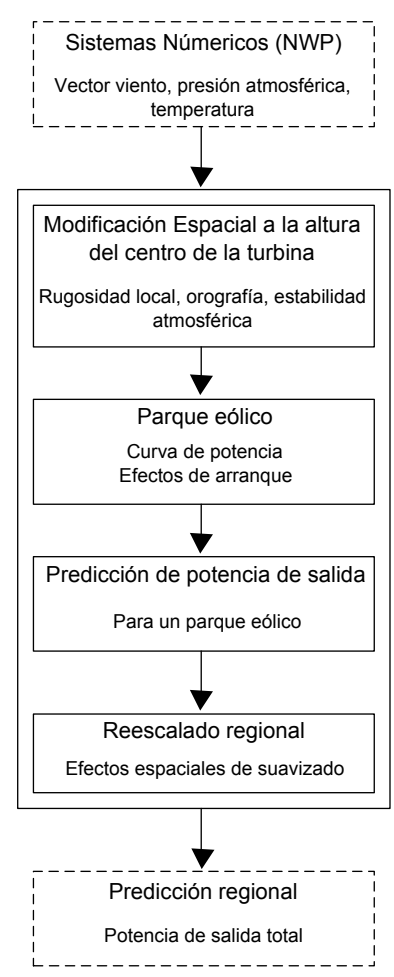

Figura 2.21: Esquema básico de Previento, sistema basado en una aproximación física. Focken y Lange (2006)

Se modifica a nivel local la entrada del sistema NWP y la velocidad del viento a la altura de la turbina se obtiene con un modelo físico. La predicción de potencia de un solo parque se calcula usando las curvas de potencia de las turbinas y considerando los efectos de arranque. La previsión de los parques representativos se reescalan considerando todos los efectos físicos de suavizado. El resultado es la predicción de la potencia de salida total de todos los parques eólicos en una determinada área.

\subsubsection{Sipreólico}

Debido al impacto que la generación de energía eólica está teniendo en el sistema de operación español, Red Eléctrica Española y la Universidad Carlos III de Madrid han desarrollado una herramienta de predicción llamada SIPREÓLICO, que predice la producción eólica total por horas para las 


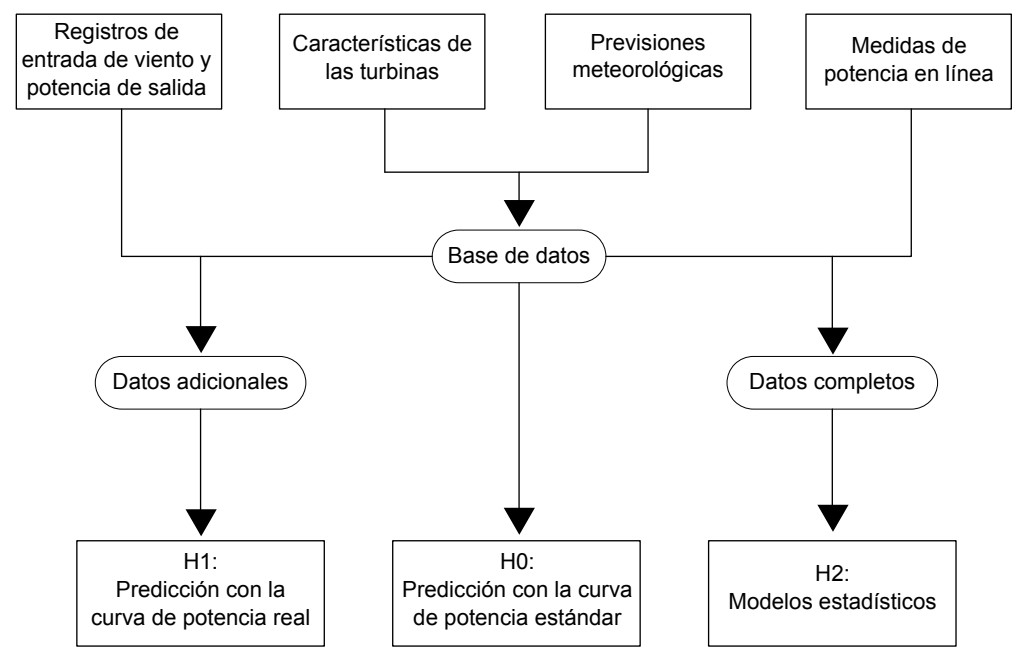

Figura 2.22: Algoritmos de predicción de Sipreólico. Gonzalez et al. (2004).

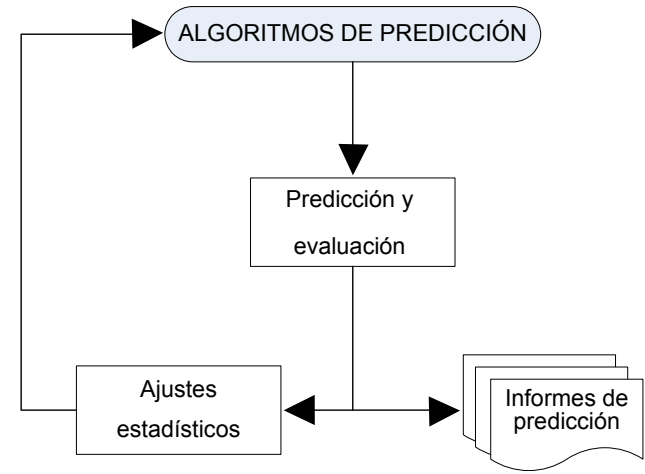

Figura 2.23: Diagrama de funcionamiento de Sipreólico. Gonzalez et al. (2004).

siguientes 48 horas.

Este modelo de predicción eólica a corto plazo fue diseñado como una herramienta de alta flexibilidad, de modo que puede realizar predicciones en función de los datos disponibles. La figura 2.22 muestra el diagrama de flujo con las principales tareas, entradas y metodologías de cálculo alternativas para el sistema. Estas alternativas dependen de la información disponible en cada parque eólico.

Una vez seleccionado el algoritmo de predicción adecuado, Sipreólico proporciona predicciones a corto plazo de la producción eólica para cada una de las unidades de predicción recogidas en una tabla de parques.

Las unidades de predicción pueden representar parques individuales o pequeñas agrupaciones de parques cercanos que vierten su potencia a un mismo 
CApítulo 2. Variabilidad y predictibilidad de la energía eólica y sus efectos sobre la red eléctrica

nudo y que comparten la información de ubicación geográfica y de medidas de generación eléctrica. Una vez obtenidas las predicciones por parques, se obtienen por zonas, compañías, etc. Del mismo modo también se calcula la predicción de potencia total peninsular como la suma de las predicciones de todos los parques.

Las predicciones de potencia eólica para las siguientes 24 a 48 horas son esenciales por lo que respecta a los sistemas de predicción a corto plazo. En ese sentido, en cada país hay un número de herramientas de predicción numéricas disponibles. Actualmente, en el caso de España, el operador del sistema, Red Eléctrica de España, recibe predicciones de Hirlam y del Centro Europeo de Predicciones (ECMWF). Sipreólico se diseñó para incorporar las previsiones de Hirlam. Diferentes organismos meteorológicos de Dinamarca, Finlandia, Islandia, Irlanda, Holanda, Noruega, Suecia y España, han colaborado en el proyecto Hirlam por medio de sus Institutos Nacionales de Meteorología, en caso de España, la Agencia Estatal de Meteorología (AEMET). Este modelo calcula predicciones de varias variables meteorológicas como la velocidad media y la dirección predominante del viento. Para el territorio Español, estas variables se proporcionan con diferentes resoluciones dependiendo del horizonte temporal seleccionado. Para un horizonte de 56 horas, la resolución espacial alcanza 0.16 grados $\left(16 \mathrm{~km}^{2}\right)$. Las predicciones se obtienen 4 veces al día (cada 6 horas). Hoy en día se evalúan otras fuentes. El modelo ECMWF produce predicciones a largo plazo (4 días) con una resolución menor ya que se ejecuta una vez por día y ofrece los valores de predicción cada 12 horas con una resolución espacial de 0.25 grados ( 25 $k m^{2}$ ).

Cuando se reciben predicciones meteorológicas de más de una agencia, Sipreólico elabora predicciones de potencia horaria usando el viento previsto de cada una de ellas. Después, se elabora una predicción de potencia final mediante la combinación de ambas predicciones.

Sipreólico está en operación desde Febrero de 2002. Entonces comenzó un proceso continuo de mejora. A pesar de que el sistema fue diseñado para ser capaz de trabajar en todos los casos mencionados en la introducción a Sipreólico, hoy en día Red Eléctrica de España se centra en el desarrollo y validación del algoritmo que usa modelos estadísticos alimentados con medidas de potencia eólica en tiempo real. De hecho, con esas medidas, el sistema está actualizado continuamente y genera mejores predicciones.

La base de datos de Sipreólico almacena información de cada parque eólico: situación geográfica, tipo de turbina eólica, curvas potencia-velocidad (P-V) teóricas y reales, series históricas de generación, etc. Esto es gestionado por el programa BDSipreólico que permite al usuario añadir, borrar, modificar o actualizar cualquier registro de un modo sencillo.

Con el fin de realizar sus cálculos, el sistema usa los siguientes datos de entrada: Previsiones meteorológicas de velocidad y dirección del viento para 
cada localización de parque eólico. Red Eléctrica recibe del Instituto Nacional de Meteorología Hirlam predicciones cada 6 horas. Otras predicciones meteorológicas (ECMWF y Metra) se evalúan para comparar su exactitud. Medidas de potencia eólica en tiempo real previstas por los parques eólicos al Centro de Control de Red Eléctrica cada 12 segundos. Estas medidas consisten en medias de 15 o 20 minutos de la potencia generada por los parques eólicos. Las telemedidas son las que definen el concepto de unidad de predicción, pudiendo ser de un solo parque o el conjunto de varios parques cercanos conectados a un mismo nudo con una telemedida común.

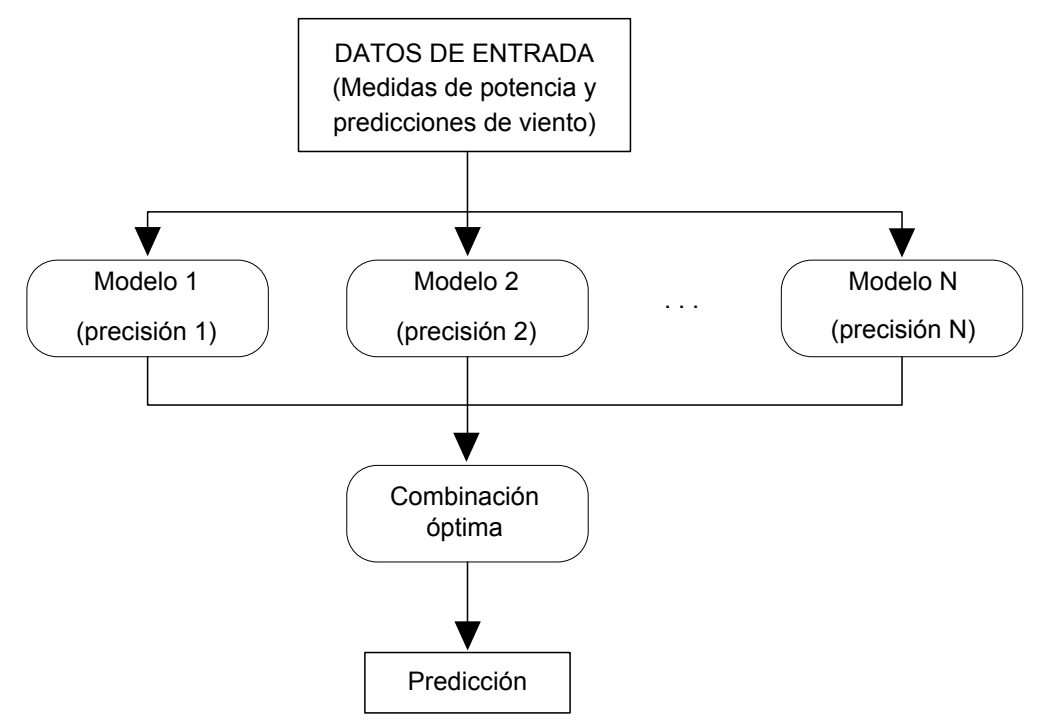

Figura 2.24: Esquema del módulo de predicción de Sipreólico. González et al. (2008).

En la actualidad, aproximadamente el $92 \%$ de la potencia eólica instalada en el Sistema Eléctrico Peninsular proporciona medidas en tiempo real González et al. (2008), por lo que Sipreólico puede realizar cálculos completos para prácticamente la totalidad de los parques eólicos, teniendo únicamente que hacer estimaciones de predicción mediante reescalado para el $8 \%$ restante.

Sipreólico usa una combinación adaptada de un conjunto de modelos estadísticos. Éste actualiza la estimación de todos los modelos en cada periodo usando la información más reciente, con lo que el modelo aprende y se actualiza a lo largo del tiempo. Sipreólico cuenta con ocho modelos diferentes, agrupados en cuatro bloques que recogen las distintas posibilidades que se plantean en cuanto a la estimación de la relación entre potencia y viento, y enfocados tanto a horizontes de muy corto plazo como a horizontes de corto plazo. La estimación de los parámetros de los modelos se hace de forma adaptativa, ya que las curvas que relacionan la producción eólica y el viento son 
CApítulo 2. Variabilidad y predictibilidad de la energía eólica y sus 62 efectos sobre la red eléctrica

variables en el tiempo en función de circunstancias cambiantes como la situación meteorológica. Por ello, los modelos y sus parámetros deben adaptarse en el tiempo en función de la nueva información que se obtenga. Además, los parámetros serán distintos en función del horizonte de predicción.

Una vez ha terminado el proceso de previsión para cada parque eólico, se obtienen 18 predicciones alternativas para un periodo de tiempo dado. La predicción final se calcula como una combinación linear de los diferentes modelos (figura 2.24).

Una vez se obtiene la predicción de generación media horaria para cada parque eólico, Sipreólico genera salidas según diferentes agrupaciones: total Peninsular, regiones (C.C.A.A. y provincias), compañías eléctricas, subestaciones, etc. Cada 15 o 20 minutos, dependiendo de la frecuencia en la recepción de medidas, Sipreólico ejecuta un cálculo de predicción eólica con resolución horaria para las siguientes 48 horas. Cada 15 o 20 minutos solo se recalculan o actualizan las predicciones para las siguientes 4 horas, y las predicciones hasta el horizonte 48 solo se calculan una vez cada hora. La figura 2.25 muestra la pantalla de salida de Sipreólico con la producción real de los últimos días y la predicción de potencia eólica para las siguientes 48 horas para la España peninsular en su conjunto.

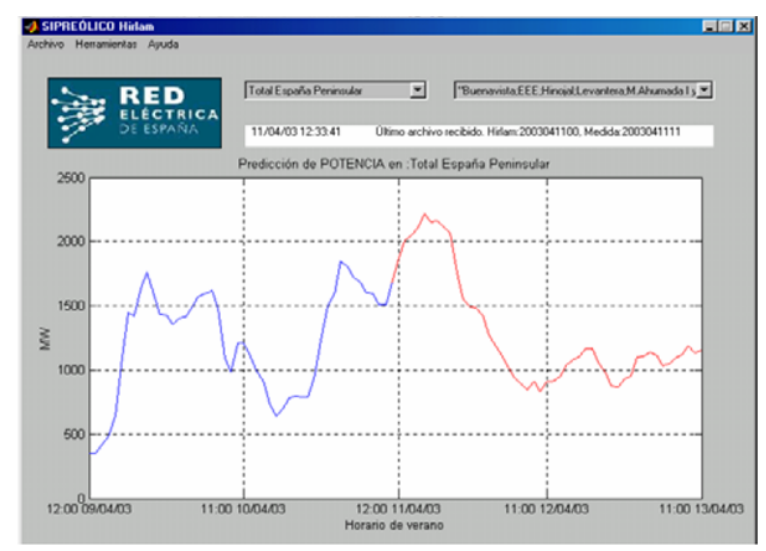

Figura 2.25: Producción de potencia eólica real para las 48 horas anteriores y predicción para las próximas 48 horas para la España peninsular en su conjunto. Gonzalez et al. (2004)

El sistema también está equipado con una herramienta de diagnóstico llamada Diagólico. Esta herramienta se ha desarrollado para evaluar la precisión de las predicciones. Esto se logra mediante el cálculo de varios parámetros estadísticos y medidas de error. Los gráficos de diagnóstico se elaboran ambos a nivel nacional y a nivel regional (C.C.A.A.), siendo este último utilizado para el análisis por zonas. 


\subsection{Efectos de la variabilidad de la energía eólica en el sistema eléctrico}

Los efectos negativos que produce la integración de generación eólica en la red eléctrica se deben principalmente a la naturaleza variable de la misma y a que, por lo tanto, es no gestionable. Estos efectos se dividen en dos grupos: efectos a corto plazo en la escala de tiempo de operación (de minutos a horas) y efectos a largo plazo teniéndose que proporcionar energía en situaciones de demanda pico. Las fluctuaciones presentadas en el capítulo anterior añaden requisitos y costes adicionales derivados de la operación del sistema.

Los principales efectos sobre la red de la variabilidad eólica se muestran en la figura 2.26, según Holttinen (2005b). Estos efectos se pueden clasificar de acuerdo a la escala espacial que afectan, desde un nivel local, pasando por un nivel regional y hasta escalas nacionales e incluso internacionales, y según la escala temporal en la que tienen vigencia, abarcando desde milisegundos hasta días e incluso años.

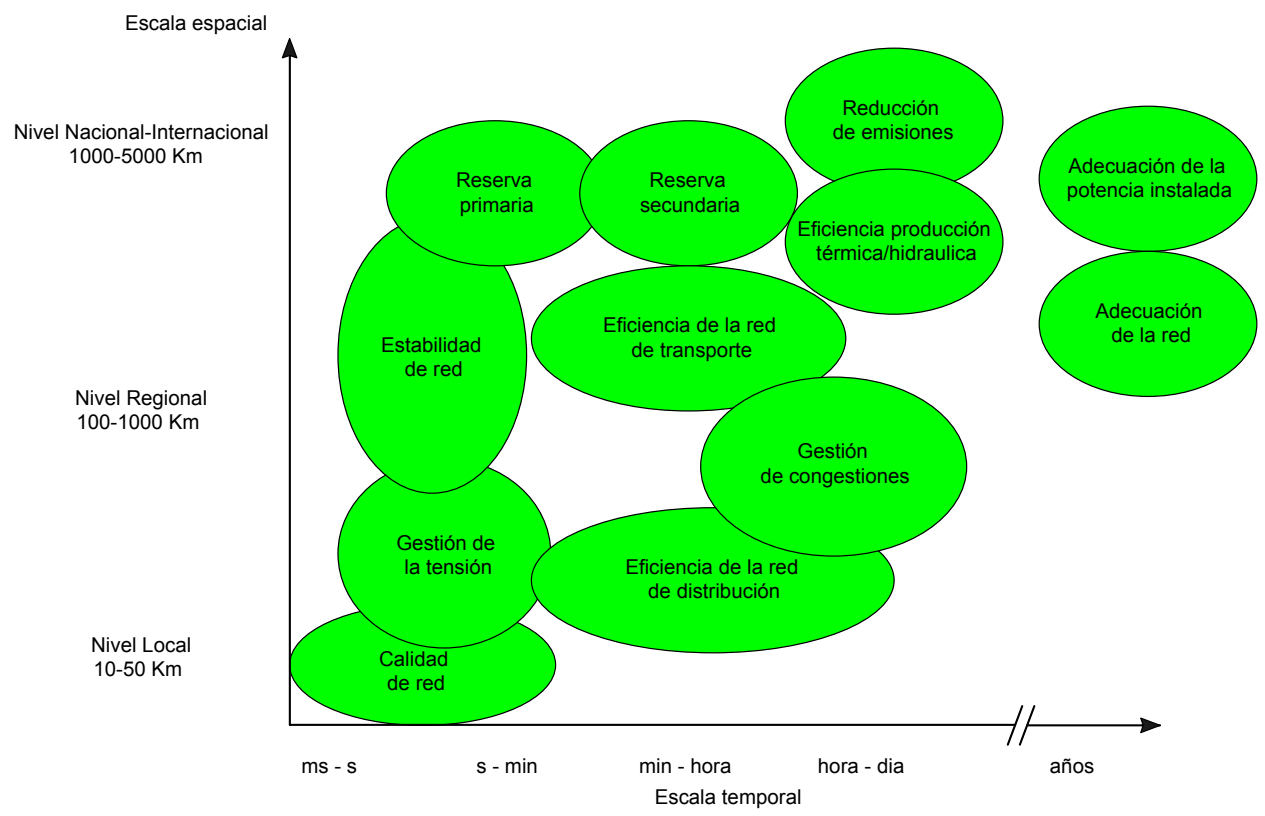

Figura 2.26: Impactos de la energía eólica sobre el sistema eléctrico. Holttinen (2005b)

Existen, además, problemas concretos según las características de los sistemas que pueden ocurrir según el nivel de penetración de energía eólica en la red, algunos de ellos son, Soder et al. (2007):

- Debe haber suficientes centrales disponibles durante periodos con una contribución eólica baja. Se puede añadir la aportación de las inter- 
CAPÍtulo 2. Variabilidad y predictibilidad de la energía eólica y sus efectos sobre la red eléctrica

conexiones internacionales a la capacidad de las centrales disponibles, aunque para sistemas aislados o con una conexión limitada se debe restringir esta disponibilidad.

- Debe haber suficientes centrales con la capacidad de generar rampas de subidas y bajadas rápidas durante situaciones con alta producción eólica. Esta disponibilidad se debe dar para mantener un balance continuo de producción y demanda. La energía rotacional que se puede almacenar en los ejes de rotación de las turbinas y generadores solo son capaces de mantener la operación del sistema durante unos segundos. En los casos de sistemas interconectados, la exportación/importación también representa un sistema rápido para aplacar esta variabilidad

- El sistema debe ser lo suficientemente robusto como para mantener la estabilidad en caso de una falta de ciertas dimensiones. En caso de sistemas interconectados esta falta puede ser transmitida desde un sistema adyacente.

- Deben estar disponibles las reservas suficientes para hacer frente a las posibles indisponibilidades de la producción y de la demanda, por ejemplo durante bajadas prolongadas de la producción eólica. En contrapunto con otro tipo de centrales que pierden toda su capacidad instantáneamente, las grandes cantidades de potencia eólica instaladas dispersas en un área relativamente grande no disminuyen su producción de forma instantánea. Esta bajada prolongada exige una prolongación en el tiempo de la disponibilidad en la capacidad de las centrales necesarias.

- Resolver posibles problemas en la red interna, asumiendo que la producción eólica se encuentra en una parte de la red y la demanda principal en otra parte. Para poder aprovechar al máximo la potencia instalada, la red de transporte debe tener una capacidad adecuada entre la producción y la demanda.

- Debe existir la posibilidad de realizar una regulación descendente de la producción eólica disponible durante periodos de baja demanda. Cuando la energía eólica cubre la mayoría de la demanda deben estar disponibles centrales que puedan variar su producción rápidamente. La producción eólica se debe poder regular para que las plantas térmicas puedan mantenerse en una carga alta incrementando su producción.

- Debe existir la posibilidad de realizar un control continuo de la producción eólica. Esta regulación se puede realizar de forma combinada con elementos de almacenamiento para reducir al máximo las perdidas.

En este capítulo se pretende establecer y analizar métodos para la optimización de la producción ya que la regulación para la estabilidad del sistema 
está plenamente desarrollada. En los siguientes apartados se desarrollan los efectos que la producción eólica ejerce sobre la generación de otras tecnologías y que aumenta los costes y el desgaste de los mismos.

\subsubsection{Efectos sobre las reservas del sistema}

En contrapunto con las rampas de potencia eólica, definidas en 2.42, se encuentran los requerimientos de rampa mediante los cuales el sistema tiene que compensar las fluctuaciones eólicas. Esto puede ser llevado a cabo regulando las plantas convencionales con rampas opuestas, suponiendo una demanda constante. Los requerimientos de la reserva, al igual que las rampas, también son un parámetro a tener en cuenta en sistemas con una alta penetración de energía eólica. Este parámetro está relacionado con las reservas de energía que deben ser dispuestas previamente, y que cuantifican la diferencia entre la potencia instantánea en el instante anterior y la potencia media en el instante actual, 2.45 .

$$
P_{\text {res }}=\overline{P_{i}(t)}-\min \left(P_{i+1}(t)\right)
$$

En este caso, los requerimientos más pronunciados se corresponden con el mayor valor de $P_{\text {res }}$ y esa caída de la energía entregada por el parque eólico debe ser cubierta por las reservas de energía de la red. Los requerimientos de reserva pueden ser cumplidos usando un sistema de almacenamiento de energía en ese espacio de tiempo dependiendo de los límites de oscilación de frecuencia fijados por el "grid code" correspondiente.

Los tipos de reservas operativas de un sistema eléctrico se clasifican en base al tipo de evento al que hacen frente, al rango de tiempo de su respuesta y a su dirección.

En el caso de eventos, algunos tipos de reservas son usadas de forma continua, mientras que otras pueden ser usadas para hacer frente a fallos instantáneos de generación o de una línea de transporte, definidos como contingencias, o a eventos prolongados y persistentes, como pueden ser eventos de rampa o errores de predicción prolongados en el tiempo.

En cuanto a su respuesta en el tiempo y las capacidades derivadas de la velocidad de respuesta, las reservas pueden ser dispuestas como generación a carga parcial para constituir una reserva rodante o como parte del control automático de la generación (AGC), el cual constituye una capacidad centralizada del operador del sistema para establecer directamente la producción deseada para cada elemento generador. Como se establece en Smith et al. (2007), la principal diferencia entre las reservas rodantes y las no rodantes es que las primeras deben estar sincronizadas con el sistema mientras que las no rodantes no están necesariamente sincronizadas. Al estar sincronizadas las reservas rodantes responden más rápidamente.

La última tipología responde a la dirección de la reserva, pudiendo ser 
CApítulo 2. Variabilidad y predictibilidad de la energía eólica y sus efectos sobre la red eléctrica

a subir o a bajar. La reserva a subir es requerida cuando la generación es inferior a la demanda existente en ese momento y por lo tanto se precisa una generación adicional o una reducción de la demanda. Por el contrario, la reserva a bajar es necesaria cuando la generación es mayor que la demanda existente y puede ser producida mediante una reducción de la generación o por un incremento de la demanda.

A partir de las características establecidas hasta el momento, se pueden definir 5 clases de reservas según Holttinen et al. (2012):

- Las reservas para respuesta en frecuencia proporcionan un respuesta frecuencial inicial ante un cambio en el balance generación/demanda. Pueden recibir otros nombres como reserva o respuesta primaria.

- Las reservas de regulación son las encargadas de mantener el control frecuencial en un área frente a cambios aleatorios en periodos de tiempo inferiores al mercado eléctrico. También se denomina control de frecuencia o reserva secundaria.

- Las reservas de rampa responden a fallos o eventos que ocurren en periodos de tiempo relativamente largos como pueden ser errores de predicción o rampas persistentes de la potencia eólica. Este tipo de reservas también son llamadas reservas para eventos de la generación variable, reservas para errores de predicción o reservas de balance.

- Las reservas para seguimiento de la demanda mantienen el control frecuencial en un área frente a cambios no aleatorios en escalas de tiempo superiores a las correspondientes para las reservas de regulación. Este tipo de reservas forma parte de las reservas terciarias.

- Las reservas suplementarias son las encargadas de reemplazar la reservas usadas para hacer frente a eventos, y mantenerlas en niveles similares a las existentes antes de los mismos. Al igual que el tipo anterior, forman parte de las reservas terciarias y también reciben los nombres de reservas de reemplazo y reservas de sustitución.

La tabla 2.6 intenta normalizar el vocabulario usado para la reservas, el cual es muy distinto en Europa y los Estados Unidos, tal y como se indica en Milligan et al. (2010). En ella se muestra un sumario de las características de los distintos tipos de reservas descritos. Durante la operación normal del sistema, se usan las reservas de regulación y las reservas para seguimiento de la demanda, mientras que cuando se produce algún tipo de evento o contingencia se activan las reservas para respuesta en frecuencia (segundos) y, para eventos en escalas de tiempo largas, las reservas suplementarias (minutos-horas). Las reservas suplementarias también actúan como "reservas de las reservas" reponiendo aquellas que han sido usadas cuando las restantes 
2.5. Efectos de la variabilidad de la energía eólica en el sistema eléctrico 67

\begin{tabular}{|c|c|c|c|c|c|}
\hline- & $\begin{array}{c}\text { Reservas para respuesta } \\
\text { en frecuencia }\end{array}$ & $\begin{array}{l}\text { Reservas de } \\
\text { regulación }\end{array}$ & $\begin{array}{l}\text { Reservas de } \\
\text { rampa }\end{array}$ & $\begin{array}{c}\text { Reservas para seguimiento } \\
\text { de la demanda }\end{array}$ & $\begin{array}{c}\text { Reservas } \\
\text { suplementarias }\end{array}$ \\
\hline \multicolumn{6}{|c|}{ Tipo de evento } \\
\hline $\begin{array}{l}\text { Eventos de } \\
\text { contingencia }\end{array}$ & $\begin{array}{r}\text { Rápida } \\
(\mathrm{s})\end{array}$ & & & & $\begin{array}{l}\text { Lenta } \\
(\min )\end{array}$ \\
\hline Continua & & $\begin{array}{l}\text { Rápida } \\
\text { (s-min) }\end{array}$ & & Lenta $(\min )$ & \\
\hline $\begin{array}{l}\text { Eventos } \\
\text { prolongados }\end{array}$ & & & $\begin{array}{l}\text { Rápida } \\
\text { (min-h) }\end{array}$ & & Lenta $(\mathrm{h})$ \\
\hline \multicolumn{6}{|l|}{$\begin{array}{l}\text { Respuesta } \\
\text { temporal }\end{array}$} \\
\hline Rodante & $\mathrm{x}$ & $\mathrm{x}$ & $\mathrm{x}$ & $\mathrm{x}$ & $\mathrm{x}$ \\
\hline No rodante & & & $\mathrm{x}$ & $\mathrm{x}$ & $\mathrm{x}$ \\
\hline AGC & $\mathrm{x}$ & $\mathrm{x}$ & & & $\mathrm{x}$ \\
\hline \multicolumn{6}{|l|}{ Dirección } \\
\hline A subir & $\mathrm{x}$ & $\mathrm{x}$ & $\mathrm{x}$ & $\mathrm{x}$ & $\mathrm{x}$ \\
\hline A bajar & $\mathrm{x}$ & $\mathrm{x}$ & $\mathrm{x}$ & $\mathrm{x}$ & \\
\hline
\end{tabular}

Tabla 2.6: Tipología de las reservas

son insuficientes para mantener el sistemas en condiciones de seguridad ante nuevos eventos. Tal y como cabría esperar, las reservas "lentas" (de rampa, para seguimiento de la demanda y suplementarias) contienen una mezcla de reservas rodantes y no rodantes, mientras que las reservas "rápidas" (para respuesta en frecuencia y de regulación) están constituidas estrictamente de reservas rodantes.

Cuando la energía eólica se integra en el sistema eléctrico, los requerimientos adicionales y los costes del balance del sistema en la escala de tiempo se deben principalmente a las fluctuaciones de la generación eólica, Zhang y Chowdhury (2009). De esta forma, la variabilidad de la producción eólica cambia el programa previsto de otras unidades de generación. Dependiendo de la precisión en la predicción, parte de los cambios en la producción no han sido previstos o tienen una predicción incorrecta. A causa de falta de seguridad en la predicción se hace necesario asegurar que exista suficiente capacidad para cubrir el incremento de las reservas.

Este incremento de las reservas necesarias en sistemas con alta penetración eólica ha sido estudiado por diferentes autores. Así, en Dany (2001) se presenta un estudio que cuantifica las consecuencias técnicas del incremento de la potencia eólica en Europa sobre las reservas primarias, secundarias y a largo plazo. Posteriormente, Suwannarat et al. (2007) propusieron modelos de plantas generadoras y sistemas de control para analizar la desviación en los intercambios internacionales en la frontera entre Alemania y Dinamarca teniendo en cuenta la naturaleza fluctuante de la potencia eólica. Así mismo, presentaron el funcionamiento del control secundario en centrales térmicas y el control de reservas rodantes en unidades de ciclo combinado para llevar a cabo el balance de potencia activa con un incremento de la penetración eólica.

Existen también otras maneras de hacer frente a la falta de suficientes 
CApítulo 2. Variabilidad y predictibilidad de la energía eólica y sus 68 efectos sobre la red eléctrica

reservas y a la variabilidad de la potencia eólica. En Yi y Rahman (2010) se propone la respuesta de la demanda como una forma efectiva de proveer al sistema de reservas que pueda requerir en ciertos casos. Esto se puede llevar a cabo usando tecnologías de comunicación y control emergentes en un entorno de "smart grid".

\subsubsection{Métodos para el cálculo del impacto de la energía eólica en las reservas.}

Para estimar el impacto sobre las reservas operacionales de la energía eólica, en Holttinen et al. (2008) se proponen dos métodos. Generalmente, el incremento relativo en las fluctuaciones del sistema debido a la potencia eólica depende del nivel de integración de la energía eólica existente en el sistema y de las características de la red. Las porciones de la energía generada por las diferentes tecnologías también son aparentemente importantes, ya que en sistemas con el mismo nivel de penetración eólico los costes derivados de la integración de la energía eólica son diferentes.

A continuación se detallan los dos métodos de cálculo de reservas en función de la potencia eólica existente.

\subsubsection{Aproximación estadística basada en la desviación están- dar.}

Se pueden aproximar las reservas necesarias si se considera que el operador del sistema debe mantener el balance entre demanda y generación y los principales focos de variabilidad que se deben estudiar son la demanda y la potencia eólica. Así, esta variabilidad se puede extraer de las series temporales de la potencia eólica y de la demanda a través de las rampas, tal y como se detalla en las siguientes ecuaciones:

$$
\Delta P_{i}=P_{i}-P_{i-1}
$$

$$
\Delta L_{i}=L_{i}-L_{i-1}
$$

siendo $P_{i}$ y $L_{i}$ los valores de demanda y potencia eólica en el instante actual, $P_{i-1}$ y $L_{i-1}$ los valores de demanda y potencia eólica en el instante anterior, y $\Delta P_{i}$ y $\Delta L_{i}$ las rampas resultantes. El incremento de la variabilidad que aporta la potencia eólica al sistema se puede apreciar cuando se compara la combinación de la demanda y la potencia eólica, llamada demanda de red (NL), con la serie temporal de la demanda. La demanda de red es la demanda existente menos la producción eólica en cada instante de tiempo de la serie temporal, representando la generación del resto de tecnologías y el resultado de los intercambios internacionales. Las variaciones de la demanda de red son calculadas como las variaciones de demanda y de potencia eólica pero 


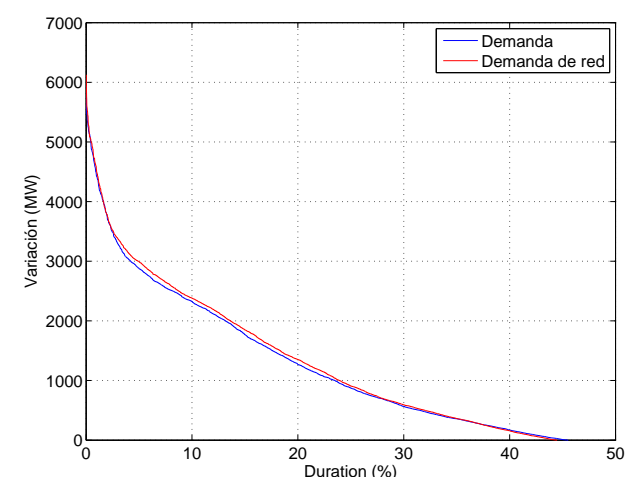

(a) Rampas positivas

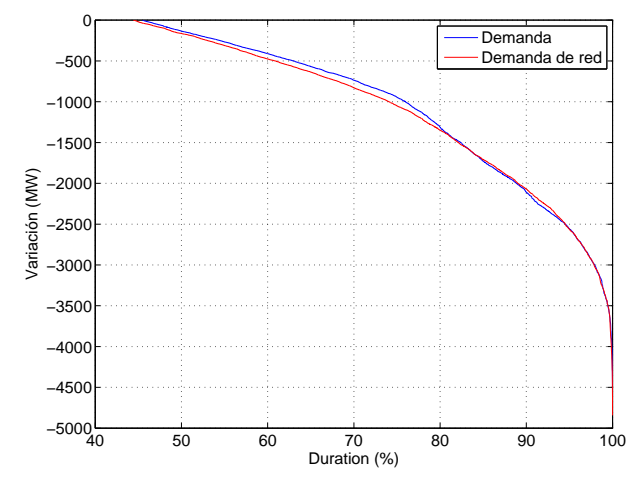

(b) Rampas negativas

Figura 2.27: Curvas de duración de las variaciones de demanda y demanda de red para el año 2009 usando medias horarias.

considerando la demanda de red como la resta entre la demanda y la potencia eólica:

$$
\Delta N L_{i}=N L_{i}-N L_{i-1}=\left(L_{i}-P_{i}\right)-\left(L_{i-1}-P_{i-1}\right)=\Delta L_{i}-\Delta P_{i}
$$

donde $\Delta N L_{i}$ es el valor de la rampa resultante para la demanda de red, mientras que $N L_{i}$ y $N L_{i-1}$ son los valores de demanda de red en el instante actual y anterior respectivamente.

En la figura 2.27 se muestran las curvas de duración de las rampas de la demanda y la demanda de red en el sistema eléctrico español durante 2009 usando medias horarias. La diferencia entre los valores máximos de rampas de demanda y demanda de red constituye el incremento necesario de la capacidad de las reservas. Así, las reservas en un sistema eléctrico generalmente se establecen de forma que la variabilidad sea cubierta con una cierta probabilidad, por ejemplo un $99 \%$ o un $99.99 \%$. La probabilidad establecida se estudia a partir de la desviación estándar, calculada en 2.38. Tal y como se describía en la sección 2.2 .2 , si se toma un rango de $\pm 3 \sigma$ se cubre un $99 \%$ de la variabilidad y aumentando el rango a $\pm 4 \sigma$ se abarca el $99.99 \%$ de todas las variaciones.

Si se considera que las series temporales de la demanda y de la potencia eólica son no correlacionadas, la desviación estándar de la demanda de red se puede calcular como la raíz cuadrada de la suma de la desviación estándar de la demanda y de la potencia eólica:

$$
\sigma_{N L}=\sqrt{\sigma_{L}^{2}+\sigma_{P}^{2}}
$$

Sin embargo existe una cierta correlación entre la demanda y la potencia eólica en ciertas regiones. Además, la distribución de las rampas normalmente 
CApítulo 2. Variabilidad y predictibilidad de la energía eólica y sus 70 efectos sobre la red eléctrica

no corresponde estrictamente con una distribución normal. Aun así, se han conseguido buenos resultados usando la ecuación 2.49 con diferentes series temporales como se desprende de Holttinen (2005b). De esta manera se puede formular el incremento de la variabilidad debido a la potencia eólica como un múltiplo del incremento de la desviación estándar, resultando:

$$
I=N \cdot\left(\sigma_{N L}-\sigma_{L}\right)
$$

$N$ denota el porcentaje de variabilidad que se pretende cubrir, siendo $N=4$ para un porcentaje del $99.99 \%$ y $N=3$ para un porcentaje de $99 \%$.

\subsubsection{Aproximación estadística basada en el nivel de excedentes.}

Esta aproximación es similar a la anterior pero en lugar de calcular los múltiplos de la desviación estándar, los cuales corresponden con bajas probabilidades que la demanda exceda la generación, el nivel de probabilidad se elige "a priori" en base a la tolerancia del riesgo asumido. Por ejemplo, si el nivel de excedentes elegido es del $99 \%$, entonces la serie temporal de la demanda es ordenada y se extrae el dato correspondiente al porcentaje del $99 \%$. En una distribución normal este valor correspondería a $3 \sigma$. Si la distribución de las series temporales no es normal, el uso del nivel de excedentes proporciona una aproximación alternativa con el mismo riesgo.

Para determinar el impacto sobre las reservas con esta aproximación, los pasos son los siguientes:

1. Cálculo del nivel de excedentes en porcentaje para la demanda.

2. Cálculo del mismo nivel de excedentes en porcentaje para la demanda de red.

3. Comparación de los niveles obtenidos, estableciendo el incremento de variabilidad.

\subsubsection{Efectos sobre la generación térmica e hidráulica.}

La fluctuaciones de la potencia eólica introducen efectos que influencian la competitividad de las centrales térmicas e hidráulicas en el sistema eléctrico. En un sistema eléctrico en el que no se aplican estrategias activas para la gestión de las fluctuaciones, estas variaciones solo pueden ser gestionadas de 3 maneras diferentes:

- Mediante la operación a carga parcial de las unidades térmicas o hidráulicas.

- Mediante el arranque o parada de unidades térmicas o hidráulicas. 
- Recortando la producción eólica.

La elección de la estrategia de gestión de las fluctuaciones depende de las propiedades de las unidades térmicas e hidráulicas que se pueden gestionar, por ejemplo, de forma que se pueda elegir una unidad a parar, esta debe estar en funcionamiento, y la duración de la variación. En sistemas donde el coste se pretende minimizar, la estrategia de gestión de las fluctuaciones asociada con el coste más bajo es la elegida. Si, por ejemplo, la producción eólica y la producción de las centrales base exceden la demanda durante una hora, el recorte de la producción eólica o posiblemente el recorte eólico en combinación con la operación a carga parcial de unidades térmicas o hidráulicas debe ser la solución asociada con un coste mínimo total para el sistema. Si la misma situación se prolonga durante 6 horas, la parada de unidades térmicas o hidráulicas es una solución más adecuada desde la perspectiva de minimización de costes. Para poder tomar una decisión en la gestión de las fluctuaciones en cuanto al despacho de unidades, es necesario conocer las características de arranque y funcionamiento a carga parcial de las unidades presentes.

Existen dos aspectos en el arranque de unidades térmicas e hidráulicas que tienen un impacto inmediato en el programa de la unidad, el tiempo de arranque y el coste de arranque. En el caso de las centrales térmicas, el tiempo de arranque se mide como el tiempo que conlleva calentar la unidad hasta que la misma alcanza un estado en el que es capaz de entregar potencia a la red, también llamado tiempo de sincronización, o como el tiempo en que sea capaz de entregar la potencia nominal a la red, denominado tiempo hasta plena carga. En ambos casos, el tiempo de arranque depende de la capacidad de la unidad, de la tecnología de la central y del tiempo durante el cual la planta ha estado parada. Las turbinas de gas pequeñas tienen tiempos de arranque relativamente cortos, en el rango de 15 minutos, mientras que turbinas grandes de vapor tienen prolongados tiempos de arranque, en el rango de varias horas. Si una unidad grande ha estado parada durante unas cuantas horas, los materiales puede que aún se mantengan calientes y el tiempo de arranque se reduce.

\begin{tabular}{lc}
\hline \multicolumn{1}{c}{ Tipo de central } & Tiempo de arranque en frió \\
\hline CC. TT. Carbon & $6-12 \mathrm{~h}$ \\
\hline CC. TT. Fuel/Gas & $5-12 \mathrm{~h}$ \\
\hline CC. TT. Ciclo Combinado & $1.5-5 \mathrm{~h}$ \\
\hline Centrales hidráulicas & $4-6 \mathrm{~min}$. \\
\hline Centrales de bombeo & $4-10 \mathrm{~min}$. \\
\hline
\end{tabular}

Tabla 2.7: Tiempos de arranque de grandes centrales 
CApítulo 2. Variabilidad y predictibilidad de la energía eólica y sus 72 efectos sobre la red eléctrica

En la tabla 2.7 aparecen los tiempos de arranque en frío de las distintas tecnologías. En ciertas centrales de carbón de más de un grupo el estado de desconexión de uno de los grupos puede conducir a tiempos de arranque de hasta 24 horas en el otro. Mientras que la baja utilización de las CC. TT. de Fuel hace que los tiempos de arranque en frío puedan alcanzar valores de hasta 24 horas debido a los requerimientos para su puesta en marcha. Por otro lado en la tabla 2.8 se definen los tiempos máximos de arranque de unidades de más de $25 \mathrm{MW}$ para el sistema eléctrico Danés definidos por Energinet Energinet (2007).

\begin{tabular}{|c|c|c|}
\hline \multirow[t]{2}{*}{ Tiempo desde la última parada } & \multicolumn{2}{|r|}{$t_{c}$} \\
\hline & {$[\mathrm{min}]$} & {$[\mathrm{min}]$} \\
\hline Inmediatamente después de la parada & 120 & 210 \\
\hline Hasta 8 horas después & 180 & 300 \\
\hline Entre 8 y 36 horas después & 300 & 480 \\
\hline Más de 36 horas después (arranque en frío) & 600 & 840 \\
\hline
\end{tabular}

Tabla 2.8: Tiempos máximo de arranque hasta sincronización $\left(t_{a}\right)$ y tiempo hasta plena carga $\left(t_{c}\right)$ de centrales con potencia nominal superior a $25 \mathrm{MW}$

Los costes asociados con el arranque de una unidad térmica son el resultado del coste del combustible requerido durante la fase de calentamiento y el componente de aceleración del envejecimiento debido a los esfuerzos producidos en los componentes de la planta a causa de los cambios de temperatura. Se ha demostrado que el efecto combinado del desgaste, debido la operación a potencia nominal, y la fatiga, debida al ciclo arranque/parada y operación con seguimiento de la demanda puede reducir sensiblemente la vida útil de los materiales presentes en la central térmica. El rango de costes viene dado también por las diferencias entre la carga nominal y la parcial durante el ciclo y el número y duración de los arranques/paradas. Estos costes incluyen la parte asociada a un mayor mantenimiento, así como al incremento del coste total del sistema por la reducción en la disponibilidad de la central y un incremento en los costes de ingeniería para adaptar las unidades a la nueva situación, denominado mejora del ciclo.

Una alternativa a la parada y arranque de la central es la reducción de la carga en una o varias unidades. La reducción de carga en una unidad está restringido por el máximo gradiente disponible de bajada y el mínimo nivel de carga. El mínimo nivel de carga viene dado por la tecnología utilizada y por el tipo de combustible usado en las unidades de combustión. El nivel mínimo de carga en el sistema eléctrico Danés abarca desde el $20 \%$ de la potencia nominal para las centrales térmicas de vapor alimentadas con gas/fuel hasta el $70 \%$ de la potencia nominal para plantas de residuos y biomasa, Energinet (2007). El nivel mínimo de carga para centrales de carbón abarca el rango entre 35 y $50 \%$ de la potencia nominal dependiendo de la tecnología. 


\begin{tabular}{|c|c|c|c|}
\hline Tecnología & $\begin{array}{l}\text { Máxima capacidad } \\
{[\mathrm{MW}]}\end{array}$ & $\begin{array}{l}\text { Mínima capacidad } \\
{[\mathrm{MW}]}\end{array}$ & $\begin{array}{l}\text { Máximo gradiente } \\
{[\mathrm{MW} / \mathrm{min}]}\end{array}$ \\
\hline $\begin{array}{l}\text { Plantas de carbón } \\
\text { nuevas }\end{array}$ & 800 & 200 & \pm 27 \\
\hline $\begin{array}{l}\text { Plantas de carbón } \\
\text { antiguas }\end{array}$ & 600 & 420 & \pm 8 \\
\hline $\begin{array}{l}\text { Ciclo combinado } \\
\text { nuevas }\end{array}$ & 875 & 260 & \pm 38 \\
\hline $\begin{array}{l}\text { Ciclo combinado an- } \\
\text { tiguas }\end{array}$ & 420 & 168 & \pm 10 \\
\hline Nucleares nuevas & 1260 & 630 & \pm 63 \\
\hline
\end{tabular}

Tabla 2.9: Capacidades y gradientes típicos de diferentes tecnologías de generación RWE (2009)

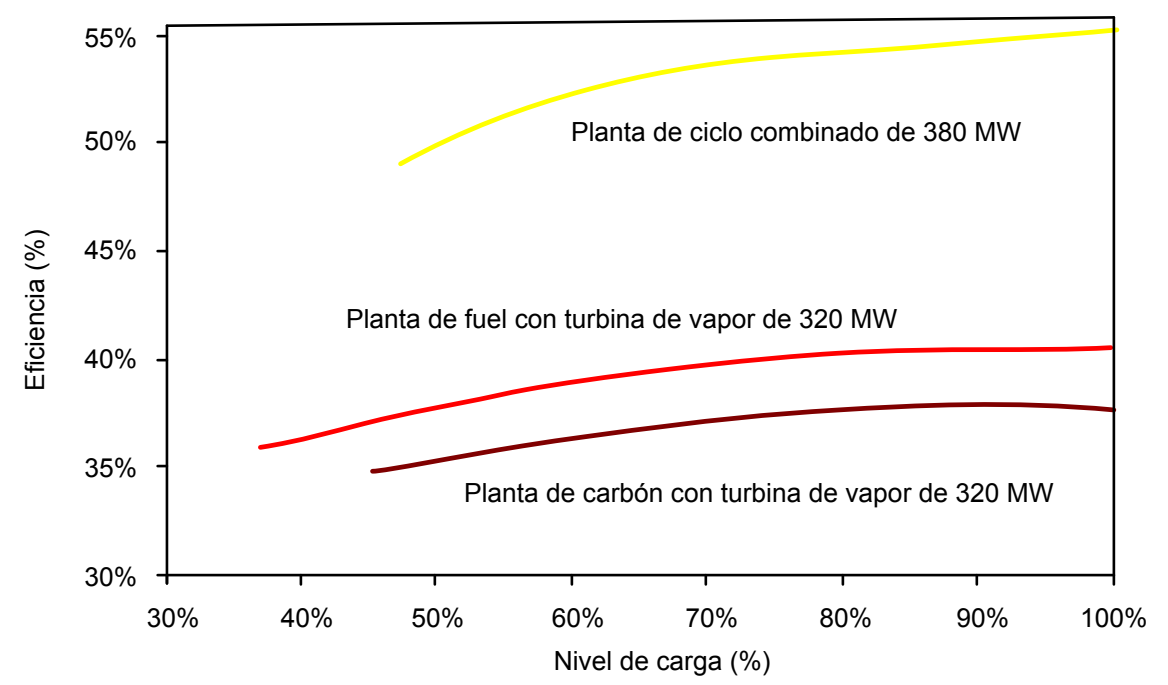

Figura 2.28: Variación de la eficiencia de las centrales térmicas con la carga Gøransson y Johnsson (2010)

Operar centrales térmicas a carga parcial conlleva asociado un incremento en costes y emisiones por unidad de energía generada, debido a que la eficiencia decrece con el nivel de carga. El grado de decrecimiento de la eficiencia depende de la tecnología de la planta y del nivel de reducción de la carga. Como se puede apreciar en la figura 2.28 , la perdida de eficiencia es menor a niveles de alta carga parcial que a niveles de baja carga parcial. También se observa que la pérdida de eficiencia es mayor en una planta de ciclo combinado que en una planta de turbina de vapor, debido a que la turbina de gas es más sensible a la operación a carga parcial.

El incremento de la potencia eólica introducida en la red reduce los periodos de producción constante de las centrales convencionales y la duración de los mismos. El factor de capacidad de unidades con un arranque y gradiente 
CApítulo 2. Variabilidad y predictibilidad de la energía eólica y sus 74 efectos sobre la red eléctrica

lento y un alto nivel mínimo de carga decrece en mayor medida que el factor de capacidad de unidades con un arranque y gradiente rápido y con un nivel mínimo de carga alto. Así se indica en Carraretto (2006). Aunque, un arranque y gradiente lento con un nivel mínimo de carga alto son propiedades comunes en las unidades con menores costes de operación que se utilizan para cubrir la base de la demanda. Por esto, los bajos costes de operación compiten con la flexibilidad de la unidad por lo que en un sistema con una cierta potencia eólica instalada la unidad térmica con los menores costes de operación no es necesariamente la que posee un factor de capacidad mayor, tal y como se indica en Gøransson y Johnsson (2010) y en RWE (2009).

Las estrategias para moderar estas pérdidas abarcan desde la regulación de la potencia eólica hasta el uso de dispositivos y tecnologías de almacenamiento para poder operar las centrales a carga nominal, donde alcanzan su mayor eficiencia. La reducción de emisiones al introducir la energía eólica en un sistema se ve parcialmente minorizada por las emisiones adicionales derivadas de la ineficiencia en la operación de las centrales térmicas a causa de las fluctuaciones de la generación eólica. Una reducción en estas fluctuaciones se puede ver reflejada mediante:

- Reducción del número de arranques/paradas.

- Reducción del tiempo de operación a carga parcial.

- Reducción del recorte de energía eólica.

Estos efectos también implican una reducción de las emisiones y una mejora en la eficiencia global del sistema. El uso de la regulación de las fluctuaciones de potencia eólica puede mejorar sensiblemente la optimización de los ciclos térmicos, reduciendo los efectos negativos descritos.

\subsubsection{Recortes de generación eólica}

En ciertas situaciones la generación eólica debe ser recortada para preservar el balance, la estabilidad y la seguridad en la operación de los sistemas eléctricos. Estos recortes de energía eólica se han incrementado en los últimos tiempos, en parte debido al incremento de la potencia eólica instalada, y en parte a la falta de una infraestructura de transporte capaz de soportarla. Adicionalmente, la construcción de parques eólicos es más fácil y rápida que la construcción de líneas de transporte y distribución. Todo esto sumado a la no correlación de la demanda y la potencia eólica resulta en situaciones donde la energía eólica debe ser recortada. Hasta la fecha, los recortes de energía eólica atienden a dos razones principales:

- La falta de capacidad de transporte y distribución disponible en ciertos momentos para incorporar una parte o la totalidad de la energía eólica 
al sistema. Así, se producirán recortes cuando las líneas de transporte y/o distribución estén saturadas o cercanas a su límite térmico.

- La presencia de una alta generación eólica en periodos de baja o mínima demanda, no pudiendo exportar este exceso de generación. Durante estos periodos, el operador del sistema puede considerar recortar la generación eólica para asegurar que existen lo recursos suficientes para garantizar suficientes reservas y con la flexibilidad adecuada de forma que se pueda hacer frente a posibles fluctuaciones, tanto de la demanda como de la generación que se puedan producir. Bajo estas condiciones, la generación eólica debe ser recortada después de reducir el resto de generación al mínimo posible y minimizar las importaciones. Este fenómeno se acentúa en sistemas aislados o islas, tal y como se detalla en De Vos et al. (2013).

Ademas de estas razones existen otras que ocurren de forma puntual y bajo ciertas condiciones como son aquellas que afectan a la seguridad del sistema y su inercia o responden a estrategias de mercado, esto se detalla en Klinge Jacobsen y Schröder (2012). Los recortes según criterios de seguridad se distinguen del resto de casos por el siguiente criterio: si un límite en la capacidad de la red no es la causa del recorte pero existen limitaciones en otros factores como pueden ser la potencia reactiva o el riesgo de un cambio rápido de la generación no gestionable, se considera un recorte por criterios de seguridad. Un ejemplo de este tipo de recortes es el usado en el sistema eléctrico español, según el cual existe un valor máximo de potencia eólica que puede ser desconectada como consecuencia de un hueco de tensión sin que afecte la seguridad del sistema. Si la potencia eólica del sistema en un momento dado hace que se supere este máximo, se debe realizar un recorte para mantener el sistema en condiciones de seguridad. Por otra parte, los recortes por estrategias de mercado responden a criterios económicos en momentos en los que pueden existir ganancias potenciales con ciertas estrategias de puja en los mercados de recortes.

Si tenemos en cuenta la voluntariedad de las empresas generadoras, se pueden clasificar los recortes como voluntarios e involuntarios. En el caso de los recortes voluntarios suelen realizarse con criterios de mercados y son avisados con bastante antelación. Sin embargo, los recortes involuntarios se incluyen dentro de la operación en tiempo real del sistema.

Actualmente, los procesos de recorte de la energía eólica se encuentran en las primeras etapas de discusión e implementación. Estos procesos difieren según el país, en función de los mecanismos de mercado presentes. Así mismo, la necesidad de recortes de la generación eólica no solo depende del nivel de penetración eólica presente en el sistema, sino también de la flexibilidad de la generación existente. De esta manera, según Fink et al. (2009), los recortes se pueden caracterizar en las siguientes categorías según los procedimientos 
CApítulo 2. Variabilidad y predictibilidad de la energía eólica y sus 76 efectos sobre la red eléctrica

que se sigan:

- Recorte como condición de la conexión de los aerogeneradores. Mediante esta categoría la energía eólica se puede recortar en cualquier momento en el que las condiciones del sistema y las características de la red lo requieran. Como ejemplo, en E. On Netz en Alemania era de obligado cumplimiento para los parques instalados que se atuvieran a recortes debido a la interconexión "Schleswig-Holstein" y "Baja Sajonia" hasta que se completase la ampliación de la misma.

- Recortes contractuales. Algunos operadores del sistema incluyen en contratos con las empresas eólicas la cantidad de energía eólica que pueden recortar sin coste alguno para ellas o a un coste reducido, previamente pactado.

- Mercado de recortes. Este tipo de categoría permite a las empresas eólicas proponer mediante un sistema de pujas un precio al que estarían dispuestas a realizar operaciones de recorte. Un ejemplo de operador del sistema que permite estas prácticas es el Operador del Sistema Independiente (ISO) de Nueva York.

- Recortes según los límites diarios de operación. En esta categoría el operador del sistema propone unos límites diarios de operación en función de las unidades de generación programadas, incluida la energía eólica, para una región dada. Esto se realiza en zonas o áreas donde existe una gran concentración de potencia instalada que puede hacer que se sature la red de transporte de la región. Así los recortes se programan a partir de esos límites. Como un ejemplo de esta categoría destacan los límites diarios de operación impuestos por el Consejo de Fiabilidad Eléctrica de Texas (ERCOT) en el oeste de Texas.

- Recortes según las diferencias entre las tecnologías de los aerogeneradores. Dentro de esta categoría los recortes se asignan en función de la respuesta de los parques de la región en cuestión. Así los parques eólicos con una respuesta rápida (máximo 15 minutos) tienen preferencia de funcionamiento sobre aquellos parques con una respuesta lenta (máximo 30 minutos), de forma que se pueda hacer frente a eventos mediante una respuesta rápida de los generadores presentes. Los parques rápidos están formados con aerogeneradores de última generación, los cuales poseen capacidades de control avanzadas.

- Recortes según la disponibilidad de reservas. En esta categoría el operador del sistema considera el recorte de la generación eólica en situaciones en las que gran parte de las reservas ya han sido utilizadas. Un ejemplo de este tipo de recortes, lo realiza la Administración de Potencia de Bonneville (BPA), la cual considera el recorte de la generación 
eólica en situaciones en las que más del $90 \%$ de las reservas disponibles de forma que no se vea comprometida la seguridad del sistema.

En la mayoría de los casos no se compensan económicamente estos recortes, tal y como se indica en Rogers et al. (2010), así ocurre en Alberta (Canadá), Nueva Zelanda y BPA. Otros casos incluyen una compensación pactada previamente mediante un contrato, como en la Compañía Eléctrica Hawaiana, o a través de un mercado de pujas (Operador del Sistema Independiente de Nueva York). En España, la compensación se establece como un porcentaje del precio de mercado en caso de recortes en tiempo real, siendo en la actualidad del $15 \%$. Por otra parte, en ciertas partes de Alemania, la cantidad compensada incluye la totalidad de los ingresos perdidos por el recorte, mientras que en Irlanda los parques eólicos recortados son remunerados a precio de mercado como si hubiesen funcionado a la máxima potencia disponible.

\subsection{Conclusiones}

En este capítulo se han expuesto las características de las fluctuaciones de la velocidad de viento con un análisis espectral y un análisis estadístico. Así mismo se han establecido las causas principales de estas fluctuaciones de potencia, su relación con las fluctuaciones de la velocidad de viento y se han evaluado los parámetros característicos de las mismas, tanto para parques eólicos individuales, como para agregaciones de los mismos de distintos tamaños, de forma que se pueda analizar el efecto de suavizado presente. Los parámetros característicos han sido calculados a partir de series temporales reales de las distintas agregaciones. Estos parámetros son utilizados por los agentes del sistema para operar el sistema de modo que se mantenga en condiciones de seguridad en todo momento.

Por otra parte se han analizado los modelos de predicción existentes, detallando dos de los ejemplos más utilizados como son el Previento y el Sipreólico. Estos sistemas se han convertido en elementos de suma importancia para conocer la evolución de la energía eólica en los sistemas eléctricos, llegando a ser la principal herramienta para hacer frente a la variabilidad de la potencia eólica.

Finalmente, se han descrito los principales efectos que produce la variabilidad de la potencia Eólica en los sistemas eléctricos. Se han destacados aquellos efectos que se producen a medio y largo plazo y que están relacionados con las reservas y el exceso de potencia eólica en la red, demostrando que la variabilidad presente en la potencia eólica influye de forma determinante en las reservas a medio y largo plazo (secundarias, terciarias y desvíos). 



\title{
Capítulo 3
}

\section{Datos utilizados}

\begin{abstract}
RESUMEN: En este capítulo se detallan los datos utilizados en el análisis de la integración eólica en la red eléctrica de España y de métodos de control propuestos para la reducción de la variabilidad de la potencia eólica. En primer lugar se detallan las principales características de los parques eólicos y de las agregaciones consideradas, así como de las series temporales utilizadas. También se describe el método de filtrado usado con los datos. En segundo y último lugar se indican los valores de los principales parámetros estadísticos para la caracterización de la variabilidad como son la desviación estándar y el PNRR-99, tal y como se han propuesto en la sección 2.3.1. Los parámetros han sido calculados para parques individuales y diversas agregaciones, y para varios rangos de tiempo (10 minutos, 30 minutos y 1 hora), de forma que se pueda ampliar el estudio del efecto del rango de tiempo escogido y el efecto de suavizado sobre dicho parámetros.
\end{abstract}

\subsection{Descripción de los datos disponibles}

En este capítulo se describen las diferentes series temporales usadas para el análisis de las fluctuaciones de potencia eólica y los métodos propuestos para su reducción. Los datos disponibles constituyen una amplia base de datos.

Se pueden establecer 5 grupos de datos en base al periodo que abarcan y al tipo de datos. El primer grupo de series temporales abarca 9 parques eólicos españoles para el año 2007. Las series temporales de este grupo corresponden a datos de potencia activa de los parques eólicos indicados. Los datos corresponden a medias diezminutales. Estos datos han sido recogidos en el SCADA del parque eólico correspondiente. En la tabla 3.1 se muestran las principales características de esos 9 parques eólicos, como son la potencia nominal del parque, el modelo o los modelos de aerogeneradores que constituyen los parques y el número de aerogeneradores de los parques. La 
situación geográfica de estos 9 parques, así como las agregaciones geográficas de estos parques consideradas en el estudio, se muestran en la figura 3.1a. Los parques se identifican como "WF" y el número de parque correspondiente, perteneciendo a este primer grupo los parques del WF1 al WF9.

El segundo grupo de series temporales incluye datos de potencia activa generada y velocidad de viento media para cada aerogenerador de 8 parques eólicos durante el año 2008. Los parques pertenecientes a este grupo son del WF10 al WF17. En el caso del parque WF13 se disponen de datos desde enero de 2007 hasta septiembre de 2010, incluyendo las velocidades máximas y mínimas de cada periodo. Al igual que en el primer grupo, los datos corresponden a medias diezminutales recogidas en el SCADA del parque eólico correspondiente. De forma similar al primer grupo, se muestran las principales características de los parques en la tabla 3.2 y la situación geográfica de los mismos se muestra en la figura $3.1 \mathrm{~b}$, incluyendo la agregaciones propuestas para este grupo al igual que se definían para el primer grupo.

El tercer grupo de datos corresponde a datos de potencia activa, velocidad de viento y dirección de viento para cada aerogenerador de un parque eólico desde Noviembre de 2011 hasta Octubre de 2012. Este parque se identifica como WF18 y sus características se muestran en la tabla 3.3. La localización geográfica de este parque se muestra en la figura 3.1b. Las series temporales de este grupo están constituidas por valores medios cada 2 segundos recogidos en el SCADA del parque eólico.

El cuarto grupo de datos incluye la producción eólica global, la producción global de otras tecnologías como la hidráulica, la de ciclo combinado y la nuclear, la demanda real y programada, reservas secundarias y terciarias, la gestión de desvíos y la predicción de potencia eólica y demanda para el sistema eléctrico español. Las series temporales de estos datos están constituidas por medias diezminutales para los casos de generación y demanda y por medias horarias para el caso de valores programados, valores de predicción y reservas. El periodo de medición para estos datos abarca desde el año 2007 al año 2012, ambos inclusive. Estos datos han sido obtenidos y recopilados por el operador del sistema eléctrico español, Red Eléctrica de España (REE), en el Centro de control eléctrico (CECOEL) y en el Centro de control para el régimen especial (CECRE).

El quinto y último grupo está constituido por series temporales de huecos de tensión registrados en el parque WF18 desde 2008 hasta 2011. Consisten en datos de tensión e intensidad con una frecuencia de muestreo de $10 \mathrm{kHz}$. Se han utilizado analizadores de redes Fluke 1760 para su registro. El esquema de conexionado utilizado en la campaña de registro se muestra en la figura 3.2. Estos datos son utilizados en el cálculo y la validación de los métodos DPLL de estimación de la frecuencia ante perturbaciones en la red, propuestos en el apéndice A. 


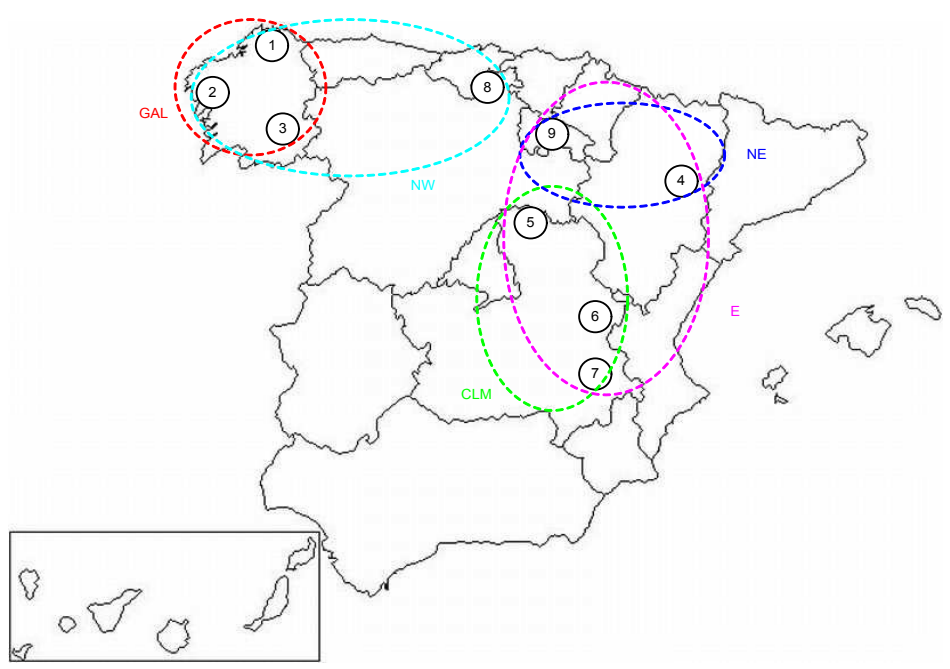

(a) 2007

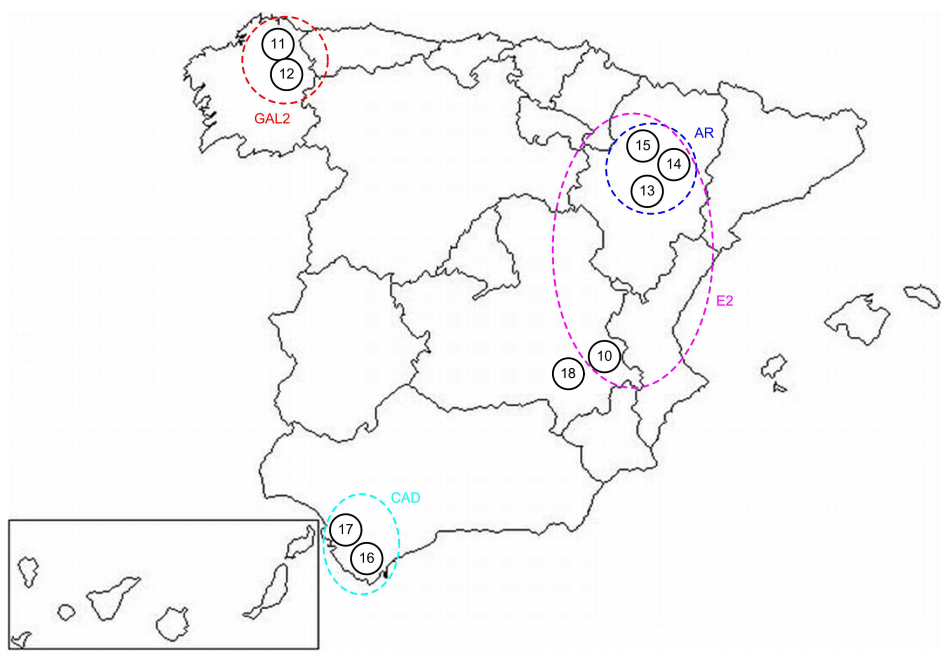

(b) 2008

Figura 3.1: Parques eólicos españoles y agregaciones consideradas.

Para caracterizar y evaluar los datos disponibles se han considerado diversas agregaciones de los parques en base a su situación geográfica o a sus características. Estas agregaciones están constituidas por parques de los dos primeros grupos de datos y se detallan en la tabla 3.4, donde se indican los parques incluidos en la agregación, la potencia total del grupo, el número de aerogeneradores del grupo, la distancia máxima entre parques del grupo y la distancia ponderada del grupo calculada según la ecuación siguiente:

$$
d_{\text {avg }}=\frac{\sum_{i}^{N} \sum_{j=i+1}^{N} d_{i j} \cdot\left(P_{i}+P_{j}\right)}{2 \cdot P_{\text {sum }}},
$$


donde $P_{\text {sum }}$ es la suma de la potencia nominal de todos los parques, $P_{i}$ es la potencia nominal del parque $W F_{i}, P_{j}$ es la potencia nominal del parque $W F_{j}$, y $d_{i j}$ es la distancia entre los parques $W F_{i}$ y $W F_{j}$.

Esta distancia ponderada se ha propuesto en base a dos parámetros importantes que influyen sobre el efecto de suavizado presente en una agregación de parques eólicos, como son la distancia entre parques y la potencia de los mismo, tal y como se indica en 2.3.3.

\subsection{Tratamiento de datos y filtrado}

Los valores utilizados en las representaciones y los cálculos realizados han sido utilizados normalmente como valores por unidad para ofrecer una cierta homogeneidad en los resultados. Las series temporales representan los valores reales medidos por lo que se incluyen periodos sin medición, o datos erróneos. También se incluyen los periodos en los que el operador del sistema ha realizado recortes sobre los parques estudiados. Los datos erróneos han sido corregidos previamente al estudio mediante un filtrado de los mismos. El filtrado de los datos se ha llevado a cabo cumpliendo el siguiente procedimiento:

- Cuando hay un valor erróneo entre dos valores correctos, este valor erróneo es sustituido por la interpolación de los dos valores adyacentes. Si hay más de un valor erróneo consecutivo entonces no se realiza filtrado y se considera que estos valores no están disponibles a la hora de realizar los cálculos de parámetros o implantar los métodos de control.

- En el análisis de agregaciones de parques, las condiciones previas deben ser cumplidas en todos los parques de forma simultánea.

Una vez realizado el filtrado, los datos restantes son analizados o usados con los diferentes métodos de control.

\subsection{Parámetros estadísticos de variabilidad de los parques eólicos y las agregaciones estudiadas.}

En esta sección se presentan los parámetros básicos característicos de las fluctuaciones de los parques eólicos y las agregaciones consideradas en 3.1. Tal y como se detallaba en el apartado 2.3.1, los parámetros más utilizados en la caracterización de la variabilidad de la potencia eólica son la desviación estándar de las rampas $\sigma$ y el PRR. Al considerar las rampas negativas como las que mayores problemas presentan, ya que es necesaria una rampa positiva de la generación convencional con la suficiente rapidez, de entre los valores PRR se ha elegido el PNRR-99 que caracteriza la rampas negativas más 


\begin{tabular}{llll}
\hline WF & Potencia & Modelo aero & Número de aeros \\
\hline 1 & $36.5 \mathrm{MW}$ & Gamesa G52 & $43(\mathrm{G} 52)$ \\
2 & $30.4 \mathrm{MW}$ & Gamesa G58/G47 & $21(\mathrm{G} 58) / 19(\mathrm{G} 47)$ \\
3 & $27.2 \mathrm{MW}$ & Gamesa G58/G52 & $19(\mathrm{G} 58) / 13(\mathrm{G} 52)$ \\
4 & $49.5 \mathrm{MW}$ & Gamesa G47 & $75(\mathrm{G} 47)$ \\
5 & $24.4 \mathrm{MW}$ & Gamesa G47 & $37(\mathrm{G} 47)$ \\
6 & $38.3 \mathrm{MW}$ & Gamesa G52 & $45(\mathrm{G} 52)$ \\
7 & $37.6 \mathrm{MW}$ & Gamesa G47 & $57(\mathrm{G} 47)$ \\
8 & $31.4 \mathrm{MW}$ & Gamesa G52 & $37(\mathrm{G} 52)$ \\
9 & $6.8 \mathrm{MW}$ & Gamesa G58/G52 & $2(\mathrm{G} 58) / 6(\mathrm{G} 52)$ \\
Sum & $282.1 \mathrm{MW}$ & - & 374 \\
\hline
\end{tabular}

Tabla 3.1: Parques eólicos estudiados en 2007

\begin{tabular}{llll}
\hline WF & Potencia & Modelo aero & Número de aeros \\
\hline 10 & $22.0 \mathrm{MW}$ & Vestas V90 & $11($ V90) \\
11 & $41.4 \mathrm{MW}$ & Vestas NM52 & $46(\mathrm{NM} 52)$ \\
12 & $28.8 \mathrm{MW}$ & Vestas NM52 & $32(\mathrm{NM} 52)$ \\
13 & $49.5 \mathrm{MW}$ & Vestas NM82 & $30(\mathrm{NM} 82)$ \\
14 & $31.4 \mathrm{MW}$ & Vestas NM82 & $19(\mathrm{NM} 82)$ \\
15 & $36.0 \mathrm{MW}$ & Vestas NM52 & $40(\mathrm{NM} 52)$ \\
16 & $6.0 \mathrm{MW}$ & Vestas V72 & $4(\mathrm{~V} 72)$ \\
17 & $12.0 \mathrm{MW}$ & Vestas V90 & $6(\mathrm{~V} 90)$ \\
Sum & $227.1 \mathrm{MW}$ & - & 188 \\
\hline
\end{tabular}

Tabla 3.2: Parques eólicos estudiados en 2008

\begin{tabular}{llll}
\hline WF & Potencia & Modelo aero & Número de aeros \\
\hline 18 & $18.0 \mathrm{MW}$ & Gamesa G90 & $9(\mathrm{G} 90)$ \\
\hline
\end{tabular}

Tabla 3.3: Parques eólicos estudiados en 2012 


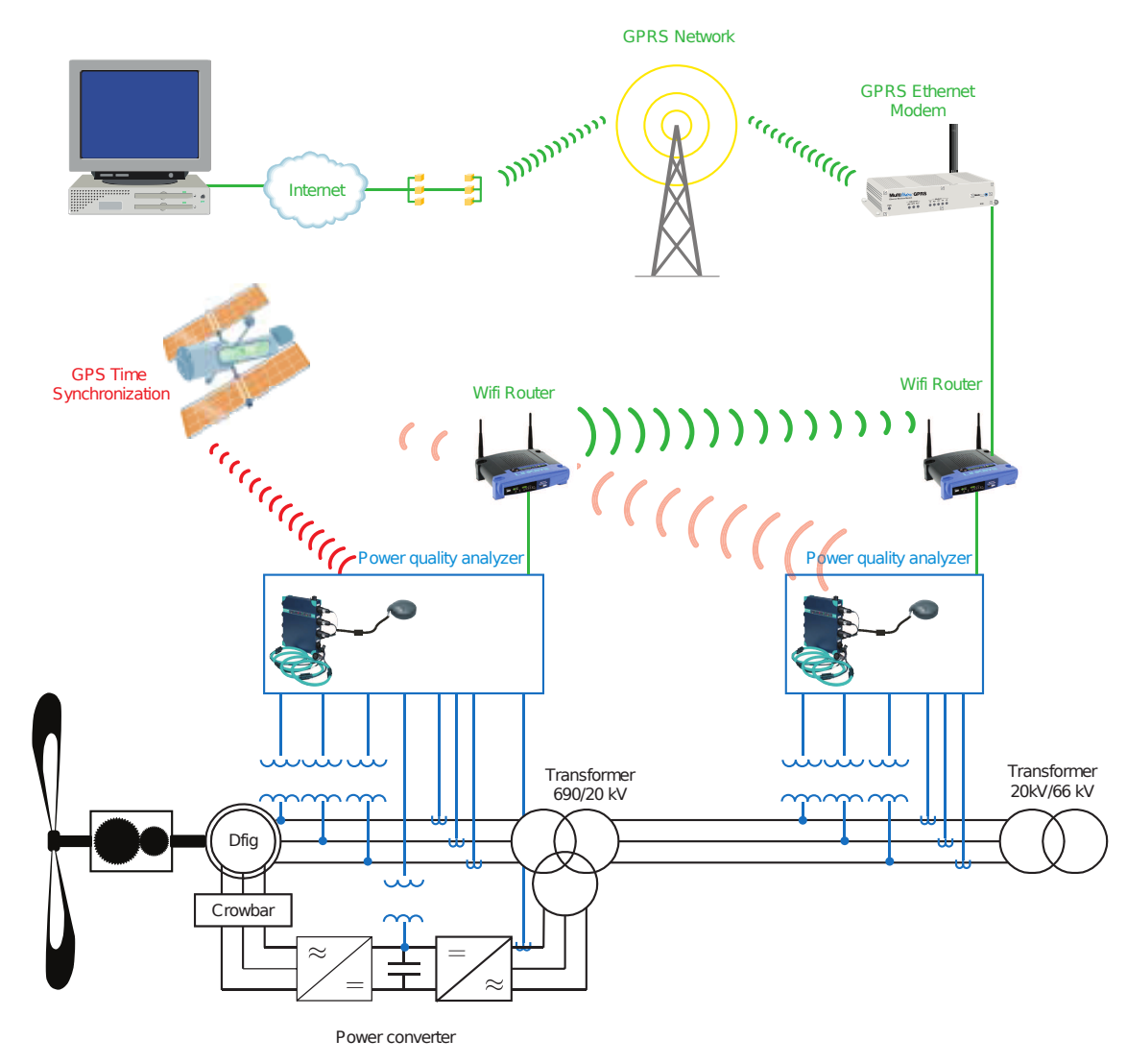

Figura 3.2: Esquema del sistema de registro utilizado para la adquisición del quinto grupo de datos. Martín-Martínez et al. (2012)

extremas. Estos parámetros y las distancias ponderadas se presentan en 3 tablas y han sido calculados usando medias de 10 minutos, medias de 30 minutos y medias de 1 hora, respectivamente. En la tabla 3.5 se incluyen los parques y agregaciones del primer grupo de datos. La tabla 3.6 incluye los parámetros de los parques y agregaciones del segundo grupo de datos usando los datos de potencia generada. Finalmente, en la tabla 3.7 se indican los parámetros de variabilidad para la potencia eólica generada en el sistema eléctrico español según los datos del cuarto grupo.

Los parámetros obtenidos son comparables por tabla ya que se refieren al mismo periodo de tiempo. Así, se observa que la variabilidad decrece según se aumenta el nivel de agregación existente, como ejemplo se puede apreciar que las rampas del parque WF1 tienen una desviación estándar de las rampas de 3,513\%/10min, este valor disminuye al considerar la agregación GAL, en la que el parque WF1 está incluido, hasta 2,243\%/10min. Si consideramos el valor de PNRR-99, la reducción es proporcionalmente similar pasando de $-0,099 \mathrm{pu} / 10 \mathrm{~min}$ para el parque WF1 a $-0,058 \mathrm{pu} / 10 \mathrm{~min}$ para la agregación GAL. Por otra parte, el valor de los parámetros de variabilidad aumenta 
Tabla 3.4: Combinaciones geográficas de parques eólicos contempladas

\begin{tabular}{llllll}
\hline \multicolumn{1}{c}{ Grupo } & WFs $^{a}$ & $\begin{array}{l}P_{n}{ }^{b} \\
(\mathrm{MW})\end{array}$ & $\begin{array}{l}\mathrm{N}^{\mathrm{o}} \\
\mathrm{aeros}^{c}\end{array}$ & $\begin{array}{l}\mathrm{de} \\
d_{\max }{ }^{d} \\
(\mathrm{Km})\end{array}$ & $\begin{array}{l}d_{\text {avg }}{ }^{e} \\
(\mathrm{Km})\end{array}$ \\
\hline GAL & $1-2-3$ & 94 & 115 & 143 & 75 \\
CLM & $5-6-7$ & 100 & 139 & 295 & 116 \\
All WFs 2007 & All 2007 & 282 & 374 & 744 & 235 \\
E & $4-5-6-7-9$ & 157 & 222 & 344 & 148 \\
NW & $1-2-3-8$ & 126 & 152 & 405 & 149 \\
\hline & & & & & \\
\hline GAL2 & $11-12$ & 70 & 78 & 22 & 22 \\
CAD & $16-17$ & 18 & 10 & 34 & 34 \\
All WFs 2008 & All 2008 & 227 & 188 & 830 & 449 \\
E2 & $10-13-14-15$ & 139 & 100 & 325 & 172 \\
ARA & $13-14-15$ & 117 & 89 & 97 & 48 \\
\hline
\end{tabular}

${ }^{a}$ Parques incluidos en el grupo

${ }^{b}$ Capacidad instalada del grupo

${ }^{c}$ Número total de aerogeneradores del grupo

${ }^{d}$ Distancia máxima entre los parques del grupo

${ }^{e}$ Distancia ponderada calculada tal y como se indica en la ecuación 3.1

cuando se contemplan rangos temporales mayores. Este efecto se distingue claramente en todos los casos planteados. Un ejemplo es el que presenta el parque WF13, usando la serie temporal con medias diezminutales se obtiene una desviación estándar de las rampas de 5,318\%/10 min, si se consideran los datos con medias de 30 minutos este valor aumenta hasta 8,727\%/30min y si se usa la serie temporal con medias de 1 hora el valor se dispara hasta $11,073 \% / 1$ hora.

En el caso de los datos de la potencia eólica total del sistema eléctrico español, se aprecia que los valores de desviación estándar de las rampas y el valor PNRR-99 no disminuyen significativamente a partir de 2007 pese a que la potencia instalada se incrementó de $12015 \mathrm{MW}$ a principios de 2007 hasta $22591 \mathrm{MW}$ a finales de 2012. Esto muestra la saturación del efecto de suavizado al alcanzar una cierta cantidad de potencia instalada en una región limitada. De esta forma se puede afirmar que la variabilidad presente en la potencia eólica total española ya apenas decrece con el nivel de agregación existente y varia ligeramente en función de las condiciones meteorológicas del año en cuestión. Por otro lado, si tenemos en cuenta los rangos temporales, la variabilidad aumenta en mayor proporción que para agregaciones más pequeñas o parques individuales, según aumentamos el periodo de las series temporales. Este efecto se reproduce de forma similar para todos los años. 
Tabla 3.5: Desviación estándar y valor PNRR-99 a partir de medias cada 10 minutos, 30 minutos y 1 hora de los parques de la tabla 3.1

\begin{tabular}{|c|c|c|c|c|c|c|c|}
\hline \multicolumn{2}{|l|}{-} & \multicolumn{2}{|c|}{ Medias 10 min } & \multicolumn{2}{|c|}{ Medias $30 \mathrm{~min}$} & \multicolumn{2}{|c|}{ Medias 1 hora } \\
\hline$\overline{W F}$ & $\begin{array}{l}\text { Dist. } \\
(\mathrm{km})\end{array}$ & $\begin{array}{l}\sigma \\
(100 \times \\
\mathrm{pu} / 10 \mathrm{~min})\end{array}$ & $\begin{array}{l}\text { PNRR-99 } \\
\text { (pu/10min) }\end{array}$ & $\begin{array}{l}\sigma \\
(100 \times \\
\mathrm{pu} / 30 \mathrm{~min})\end{array}$ & $\begin{array}{l}\text { PNRR-99 } \\
\text { (pu/30min) }\end{array}$ & $\begin{array}{l}\sigma \\
(100 \times \\
\mathrm{pu} / \mathrm{h})\end{array}$ & $\begin{array}{l}\text { PNRR-99 } \\
(\mathrm{pu} / 1 \mathrm{~h})\end{array}$ \\
\hline WF1 & 0 & 3.513 & -0.099 & 5.433 & -0.159 & 7.278 & -0.212 \\
\hline WF2 & 0 & 3.675 & -0.099 & 5.830 & -0.162 & 7.970 & -0.220 \\
\hline WF3 & 0 & 3.424 & -0.108 & 5.441 & -0.172 & 6.889 & -0.219 \\
\hline WF4 & 0 & 4.333 & -0.121 & 7.544 & -0.215 & 9.521 & -0.283 \\
\hline WF5 & 0 & 4.512 & -0.132 & 7.160 & -0.211 & 8.738 & -0.276 \\
\hline WF6 & 0 & 3.621 & -0.100 & 5.850 & -0.168 & 8.278 & -0.227 \\
\hline WF7 & 0 & 3.909 & -0.112 & 6.407 & -0.184 & 8.278 & -0.249 \\
\hline WF8 & 0 & 3.959 & -0.117 & 6.505 & -0.194 & 8.686 & -0.257 \\
\hline WF9 & 0 & 5.243 & -0.160 & 7.708 & -0.233 & 9.752 & -0.285 \\
\hline WFs $1+4$ & 605 & 2.878 & -0.078 & 4.817 & -0.133 & 6.244 & -0.173 \\
\hline WFs $4+6$ & 259 & 2.922 & -0.082 & 4.953 & -0.138 & 6.321 & -0.190 \\
\hline NE & 132 & 3.879 & -0.107 & 6.662 & -0.189 & 8.564 & -0.253 \\
\hline GAL & 130 & 2.243 & -0.058 & 3.346 & -0.092 & 4.746 & -0.124 \\
\hline CLM & 195 & 2.451 & -0.065 & 3.855 & -0.106 & 5.641 & -0.144 \\
\hline WFs $1+4+6$ & 504 & 2.318 & -0.062 & 3.803 & -0.102 & 5.817 & -0.141 \\
\hline $\mathrm{E}$ & 235 & 2.208 & -0.051 & 3.489 & -0.084 & 4.978 & -0.128 \\
\hline NW & 237 & 2.039 & -0.055 & 3.081 & -0.094 & 4.443 & -0.114 \\
\hline 9 parques & 400 & 1.703 & -0.038 & 2.439 & -0.063 & 4.068 & -0.087 \\
\hline
\end{tabular}

Un ejemplo es el año 2009 donde la desviación estándar de las rampas tiene un valor de 0,407\%/10 min con medias diezminutales y esta aumenta has 0,817\%/30min con medias de 30 minutos. Adicionalmente se puede apreciar que en el año 2012, pese a tener un valor de desviación estándar de las rampas con medias diezminutales menor que otros años, como 2007 y 2008 , para periodos de 30 minutos y 1 hora los valores de desviación estándar de las rampas y de PNRR-99 son los mayores de todos los años estudiados. 
Tabla 3.6: Desviación estándar y valor PNRR-99 a partir de medias cada 10 minutos, 30 minutos y 1 hora de los parques de la tabla 3.2 y la tabla 3.3

\begin{tabular}{|c|c|c|c|c|c|c|c|}
\hline \multicolumn{2}{|l|}{-} & \multicolumn{2}{|c|}{ Medias $10 \mathrm{~min}$} & \multicolumn{2}{|c|}{ Medias $30 \mathrm{~min}$} & \multicolumn{2}{|c|}{ Medias 1 hora } \\
\hline WF & $\begin{array}{l}\text { Dist. } \\
(\mathrm{km})\end{array}$ & $\begin{array}{l}\sigma \\
(100 \times \\
\mathrm{pu} / 10 \min )\end{array}$ & $\begin{array}{l}\text { PNRR-99 } \\
(\mathrm{pu} / 10 \mathrm{~min})\end{array}$ & $\begin{array}{l}\sigma \\
(100 \times \\
\mathrm{pu} / 30 \min )\end{array}$ & $\begin{array}{l}\text { PNRR-99 } \\
(\mathrm{pu} / 30 \mathrm{~min})\end{array}$ & $\begin{array}{l}\sigma \\
(100 \times \\
\mathrm{pu} / \mathrm{hh})\end{array}$ & $\begin{array}{l}\text { PNRR-99 } \\
(\mathrm{pu} / 1 \mathrm{~h})\end{array}$ \\
\hline WF10 & 0 & 5,198 & $-0,142$ & 7,562 & $-0,230$ & 9,526 & $-0,275$ \\
\hline WF11 & 0 & 3,848 & $-0,110$ & 5,914 & $-0,172$ & 7,890 & $-0,227$ \\
\hline WF12 & 0 & 3,743 & $-0,107$ & 5,763 & $-0,166$ & 7,582 & $-0,220$ \\
\hline WF13 & 0 & 5,318 & $-0,161$ & 8,727 & $-0,269$ & 11,073 & $-0,340$ \\
\hline WF14 & 0 & 4,730 & $-0,136$ & 8,011 & $-0,246$ & 10,606 & $-0,317$ \\
\hline WF15 & 0 & 4,153 & $-0,121$ & 7,227 & $-0,212$ & 9,644 & $-0,290$ \\
\hline WF16 & 0 & 5,685 & $-0,181$ & 7,805 & $-0,248$ & 9,704 & $-0,309$ \\
\hline WF17 & 0 & 6,868 & $-0,222$ & 7,141 & $-0,227$ & 8,426 & $-0,265$ \\
\hline ARA & 48 & 2,927 & $-0,085$ & 5,097 & $-0,148$ & 6,993 & $-0,199$ \\
\hline $\mathrm{E} 2$ & 172 & 2,595 & $-0,075$ & 4,451 & $-0,127$ & 6,110 & $-0,171$ \\
\hline GAL2 & 22 & 3,556 & $-0,105$ & 5,615 & $-0,162$ & 7,510 & $-0,217$ \\
\hline CAD & 34 & 5,056 & $-0,164$ & 5,874 & $-0,178$ & 7,346 & $-0,224$ \\
\hline 8 parques & 449 & 1,973 & $-0,055$ & 3,256 & $-0,090$ & 4,454 & $-0,116$ \\
\hline WF18 & 0 & 4,966 & $-0,151$ & 7,840 & $-0,235$ & 9,915 & $-0,292$ \\
\hline
\end{tabular}

Tabla 3.7: Desviación estándar y valor PNRR-99 a partir de medias cada 10 minutos, 30 minutos y 1 hora de la generación eólica total del sistema eléctrico español para los años 2007, 2008, 2009, 2010, 2011 y 2012.

\begin{tabular}{cllllllll}
\hline \multicolumn{1}{c}{-} & \multicolumn{4}{c}{ Medias 10 min } & \multicolumn{3}{c}{ Medias 30 min } & \multicolumn{2}{c}{ Medias 1 hora } \\
\hline WF & $\begin{array}{l}\text { Dist. } \\
(\mathrm{km})\end{array}$ & $\begin{array}{l}\sigma \\
(100 \times \\
\mathrm{pu} / 10 \mathrm{~min})\end{array}$ & $\begin{array}{l}\text { PNRR-99 } \\
(\mathrm{pu} / 10 \mathrm{~min})\end{array}$ & $\begin{array}{l}\sigma \\
(100 \times \\
\mathrm{pu} / 30 \mathrm{~min})\end{array}$ & $\begin{array}{l}\text { PNRR-99 } \\
(\mathrm{pu} / 30 \mathrm{~min})\end{array}$ & $\begin{array}{l}\sigma \\
(100 \times \\
\mathrm{pu} / \mathrm{h})\end{array}$ & $\begin{array}{l}\text { PNRR-99 } \\
(\mathrm{pu} / 1 \mathrm{~h})\end{array}$ \\
\hline España 2007 & 397 & 0,467 & $-0,0112$ & 0,903 & $-0,0228$ & 1,542 & $-0,0383$ \\
España 2008 & 397 & 0,484 & $-0,0112$ & 0,964 & $-0,0233$ & 1,621 & $-0,0392$ \\
España 2009 & 397 & 0,407 & $-0,0102$ & 0,871 & $-0,0214$ & 1,526 & $-0,0357$ \\
España 2010 & 397 & 0,429 & $-0,0106$ & 0,927 & $-0,0233$ & 1,610 & $-0,0409$ \\
España 2011 & 397 & 0,362 & $-0,0090$ & 0,820 & $-0,0201$ & 1,467 & $-0,0352$ \\
España 2012 & 397 & 0,414 & $-0,0101$ & 0,955 & $-0,0234$ & 1,721 & $-0,0417$ \\
\hline
\end{tabular}





\title{
Capítulo 4
}

\section{Características y efectos de la variabilidad de la potencia eólica en España}

\begin{abstract}
RESUMen: En este capítulo se muestra la evolución de las fluctuaciones de potencia eólica en los últimos años en el sistema eléctrico español. Se estudian los cambios que han sido necesarios para adaptarse a la creciente potencia eólica instalada. A continuación se analizan los parámetros de variabilidad característicos del sistema eléctrico en los últimos años. También se indica la evolución de la predicción eólica, sus errores y la consecuente repercusión en la reservas del sistema. Finalmente se describen eventos extremos relacionados con la potencia eólica de diversas clases que han ocurrido recientemente en el sistema eléctrico español.
\end{abstract}

\subsection{Introducción}

En los últimos años el sistema eléctrico español ha tenido que adaptarse tanto legislativamente como de forma operativa y tecnológica al crecimiento de la energía eólica. Se han desarrollado nuevos procedimientos de operación de obligado cumplimiento en estas instalaciones para afrontar los retos que ofrece esta tecnología. El cálculo de reservas, la normativa frente a huecos de tensión y la predicción eólica han evolucionado para hacer posible la integración de la energía eólica. A continuación se describen los cambios sufridos en estos campos.

Los procedimientos de operación y otros textos de obligado cumplimiento que han variado o cambiarán para adaptarse a la evolución de la energía eólica en el sistema eléctrico español, se muestran en Martín-Martínez et al. (2012) y son las siguientes: 
Capítulo 4. Características y efectos de la variabilidad de la potencia 90 eólica en España

- Procedimientos de Operación que se aplican en parte a la energía eólica y a otros tipos de energías renovables:

- PO 3.2. Gestión restricciones técnicas (D-1, en tiempo real, ...).

- PO 8.2. Operación del sistema de producción y transporte.

- PO 9. Información intercambiada con el operador de sistema (observabilidad).

- Procedimientos de Operación que se aplican específicamente a la energía eólica y a otras energías renovables:

- RD 661/2007. Aspectos de control de Tensión.

- PO 3.7. Controlabilidad de centrales de energías renovables no gestionables.

- PO 12.3. Requisitos de respuesta frente a huecos de tensión de las instalaciones eólicas.

- En proceso de resolución:

- PO 12.2. Requerimientos para nuevas instalaciones.

- PO 7.5. Control de Tensión para generación renovable.

- ENTSO-e. Los operadores del sistema de la red eléctrica europea has estudiado ciertos requerimientos para unidades de generación, los cuales serán propuestos a la comisión europea. Así se establecerán los requerimientos máximos para los operadores del sistema y los requerimientos mínimos para las unidades de generación.

Un punto crítico a tener en cuenta son las reservas del sistema para hacer frente a la generación no gestionable, como es la energía eólica. En España, las reservas de energía necesarias para hacer frente a los desequilibrios entre generación y consumo se recogen en el Procedimiento de Operación 1.5 del Operador del Sistema, REE (2006). Este Procedimiento establece tres tipos de reserva, uno para cada uno de los tres niveles de regulación.

- Reserva de regulación primaria: Se define la banda de regulación primaria del sistema como el margen de potencia en el que el conjunto de los reguladores de velocidad pueden actuar de forma automática y en los dos sentidos, como consecuencia de un desvío de frecuencia. El Operador del Sistema determina cada año los requerimientos de regulación primaria para el sistema eléctrico. La regulación primaria de los grupos generadores debe permitir un estatismo en sus reguladores de manera que puedan variar su carga en un $1,5 \%$ de la potencia nominal. Cada una de las áreas de control establecidas en el sistema europeo interconectado de UCTE ha de colaborar a la reserva de regulación 
primaria establecida para el conjunto, en función de un coeficiente de participación, que establece anualmente para cada uno de los sistemas. De este modo, para cada una de las áreas de control establecidas en el sistema europeo interconectado de la UCTE la reserva de regulación primaria exigida $(R P)$, en un año concreto, viene determinada por la siguiente expresión:

$$
R P=\frac{E}{E_{t}} \cdot R P_{t}
$$

- Reserva de regulación secundaria: Se define la banda de regulación secundaria del sistema como el margen de variación de la potencia en que el regulador secundario puede actuar automáticamente y en los dos sentidos, partiendo del punto de funcionamiento en que se encuentre en cada instante. Viene dada por la suma, en valor absoluto, de las contribuciones individuales de los grupos sometidos a este tipo de regulación. El margen de potencia en cada uno de los dos sentidos se conoce como reserva o banda a subir o a bajar.

La reserva que debe mantenerse en regulación secundaria es determinada por el Operador del Sistema para cada período de programación, en función de la indeterminación estadística en la evolución temporal previsible de la demanda y del fallo probable esperado, según la potencia y los equipos generadores acoplados. Además, tiene en cuenta la magnitud de los escalones horarios de potencia inherentes a la programación de las unidades de producción.

El comienzo de la actuación de la regulación secundaria no deberá demorarse mas allá de 30 segundos y deberá tener la capacidad de mantenerse durante un tiempo de 15 minutos hasta que su uso neto sea sustituido por la regulación terciaria. La respuesta dinámica exigible a las zonas de regulación viene definida en el procedimiento de operación 7.2 por el que se regula la prestación del servicio de regulación secundaria.

Habitualmente, en el Sistema Eléctrico Peninsular se toma como referencia la recomendación de la UCTE, Unión for the Coordination of Transmission of Electricity, según la cual el valor de la reserva secundaria debe ser como mínimo:

$$
R=\sqrt{a L_{\max }+b^{2}}-b
$$

donde $L_{\max }$ es la demanda prevista en el área de control correspondiente, $a=10 \mathrm{MW}$ y $\mathrm{b}=150 \mathrm{MW}$. La reserva secundaria a bajar se establece, en función de las condiciones de operación, entre el 40 y el $100 \%$ de la reserva a subir. 
CApítulo 4. Características y efectos de la variabilidad de la potencia eólica en España

Las reglas de la UCTE recomiendan también que, en caso de que el valor de la reserva de regulación secundaria a subir no sea suficiente para cubrir la pérdida máxima de producción asociada a un fallo simple, deberá preverse la existencia en el sistema de reserva de regulación terciaria suficiente para asegurar una respuesta rápida del sistema frente a este fallo.

Además de las recomendaciones de la UCTE, el Operador del Sistema tendrá en cuenta estas otras consideraciones a la hora de calcular los requerimientos de reserva secundaria a subir y a bajar:

- Carácter peninsular de nuestro sistema que hace necesario minimizar los desvíos en el intercambio con el resto de la UCTE, para garantizar la seguridad del sistema.

- Variación de la demanda debido a los diferentes periodos tarifarios a lo largo del día.

- Se dotará un mayor volumen de reserva en las horas que presentan puntos de inflexión de la curva de demanda peninsular.

- Así, se tendrá en cuenta en los requerimientos de reserva la posibilidad de desplazamiento en el tiempo de estos puntos de inflexión, respecto a las previsiones del OS.

- Se dotará mayor volumen de banda secundaria en aquellas horas en las que se presenten cambios acusados de programa en los intercambios internacionales. Se garantizarán los siguientes valores mínimos de banda secundaria, especialmente en horas valle, en las que, debido al parque generador conectado, existe una menor disponibilidad de reserva de regulación terciaria:

- Banda a subir: $500 \mathrm{MW}$

- Banda a bajar: $400 \mathrm{MW}$

- El Operador del Sistema, ante situaciones especiales, como eventos de interés público, condiciones climatológicas adversas, paros generales, huelgas sectoriales, etc, tomará las medidas necesarias para garantizar la disponibilidad de la reserva necesaria, aplicando, si lo considera necesario, criterios más estrictos que los descritos en los puntos anteriores.

- Reserva de regulación terciaria: Está constituida por la variación máxima de potencia a subir o a bajar de los grupos del sistema que puede ser movilizada en un tiempo inferior a quince minutos con objeto de reconstituir la reserva de regulación secundaria. La reserva mínima necesaria de regulación terciaria en cada período de programación es, como referencia, igual a la potencia del mayor grupo de generación acoplado mayorada en un $2 \%$ de la demanda prevista en cada hora. 
- Reserva programable mediante el mecanismo de gestión de desvíos: Además de las reservas anteriores de regulación primaria, secundaria y terciaria, será necesario disponer de una reserva adicional de potencia activa, programable, en su caso, mediante el mecanismo de gestión de desvíos, reserva que será cuantificada en base a la consideración de los siguientes aspectos:

- Diferencias existentes entre la demanda horaria prevista por el Operador del Sistema y la demanda horaria resultante del programa base de funcionamiento y, en su caso, de las sucesivas sesiones del mercado intradiario.

- Diferencias entre la suma de los programas de producción eólica resultantes del programa base de funcionamiento y, en su caso, de las diferentes sesiones del mercado intradiario y la producción eólica horaria prevista por el Operador del Sistema que tiene un margen de confianza equivalente al considerado para la demanda horaria prevista al establecer la reserva de regulación terciaria.

- Situaciones en las que la previsión de pérdida de generación debida a fallos sucesivos y/o retrasos en el acoplamiento o subida de carga de grupos térmicos, con probabilidad mayor o igual al $5 \%$, sea superior a la pérdida máxima de producción provocada de forma directa por el fallo simple de un elemento del sistema eléctrico.

El valor de esta reserva adicional de potencia activa vendrá determinado por:

- Reserva a subir: la suma del déficit de potencia activa generada respecto a la demandada que se deriven de la consideración de los tres aspectos anteriores.

- Reserva a bajar: la suma de los excesos de potencia activa generada respecto a la demandada que se deriven de la consideración de los tres aspectos anteriores.

El incremento de la potencia eólica instalada en las redes eléctricas en los últimos años ha hecho que haya aumentado la preocupación de los involucrados en el sistema eléctrico por los posibles problemas de estabilidad que este tipo de generación pudiese provocar. Anteriormente, los aerogeneradores no contribuían en el control y apoyo del sistema. Las variaciones de tensión y/o frecuencia en el punto de conexión del parque eólico, provocadas por faltas en la red, hacían que todos los aerogeneradores del parque fueran desconectados. Los umbrales de tensión y frecuencia establecidos para producir el disparo del parque eólico hacían que los parques eólicos desconectasen incluso con faltas alejadas de su punto de conexión. En caso de seguir con este 
Capítulo 4. Características y efectos de la variabilidad de la potencia 94 eólica en España

modo de operación, con la potencia eólica instalada actualmente, la potencia desconectada en caso de faltas en la red podría afectar a la estabilidad del sistema.

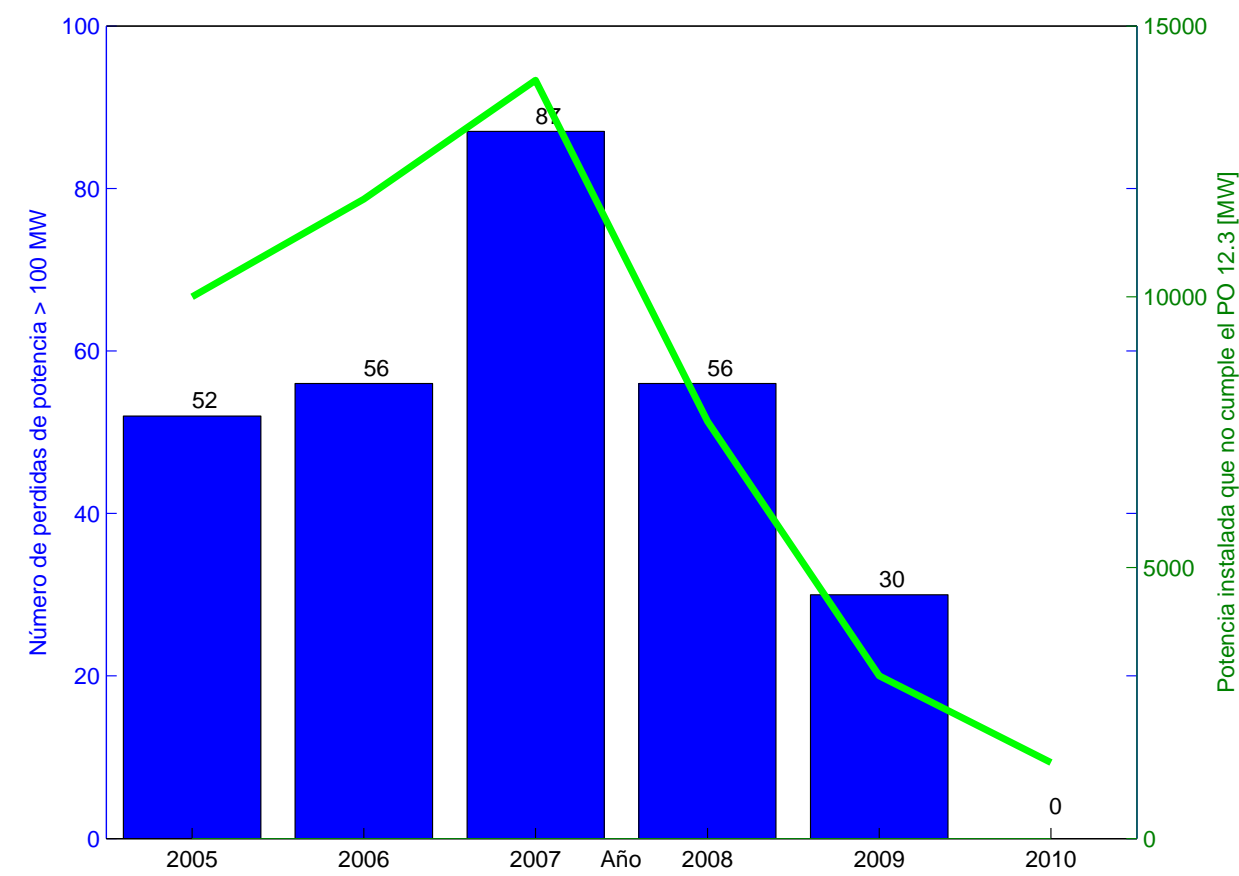

Figura 4.1: Evolución de la potencia eólica instalada con continuidad de suministro tras hueco de tensión y número de pérdidas de potencia mayores o iguales a $100 \mathrm{MW}$ por huecos de tensión en la red eléctrica española.

Para prevenir estas situaciones, los operadores del sistema han desarrollado nuevos requisitos de conexión para los generadores eólicos que incluyen la continuidad de suministro tras el hueco de tensión (o "fault ride through") e incluso la contribución a recuperar los niveles de tensión.

La principal causa de inestabilidad de tensión está relacionada con el déficit de control de energía reactiva en la red. En los parques eólicos formados por generadores de velocidad fija, cuando éstos están a plena potencia, pueden alcanzar fácilmente sobrevelocidad durante las faltas en la red. Cuando la falta es despejada, esta sobrevelocidad resulta en una absorción excesiva de potencia reactiva por parte del generador que, a su vez, puede provocar una caída de tensión. Si el generador permanece conectado a la red, esta caída incontrolada de tensión puede resultar en un colapso de tensión. Por otra parte, para evitar problemas de estabilidad en las redes con gran penetración eólica, los aerogeneradores deben participar en el control de frecuencia, ya que durante su operación normal, los cambios de carga provocan variaciones en la frecuencia.

En la resolución del 4 de octubre de 2006, se aprobó el Procedimiento de 
Operación 12.3 "Requisitos de respuesta frente a huecos de tensión de las instalaciones eólicas" en el que se regula el comportamiento que estos sistemas deben tener ante los huecos de tensión. Uno de los aspectos más relevantes tratados en los nuevos procedimientos de operación es la continuidad de suministro de los generadores eólicos durante y después de los huecos de tensión. Desde la entrada en vigor de esta normativa hasta la actualidad se ha producido un proceso de adaptación de las instalaciones eólicas para hacer frente a los huecos de tensión. En este Procedimiento de Operación se indica a este respecto: "Las instalaciones de generación no deben desconectar como consecuencia de los huecos de tensión asociados a cortocircuitos correctamente despejados; se tomarán, por lo tanto, las medidas de diseño y/o control necesarias en éstas (y todos sus componentes) para que soporten sin desconexión huecos de tensión trifásicos, bifásicos o monofásicos, en el punto de conexión a la red de transporte". Esto exige una continuidad de suministro.

Los parámetros de la red que pueden verse afectados por algún tipo de perturbación son: la frecuencia, principalmente, la amplitud, la forma de onda y la simetría del sistema trifásico. Las perturbaciones pueden producir afecciones en estos parámetros de forma periódica o de forma transitoria, es decir, sin ningún tipo de repetición. Su duración puede ser de fracciones de ciclo a varios segundos o incluso minutos. Las perturbaciones en la red suelen producirse por cortocircuitos, conexiones/desconexiones de grandes cargas y fenómenos meteorológicos (rayos). Mientras que las consecuencias de las mismas pueden ser:

- Pérdidas de elementos de la red por despeje de faltas, dependiendo de la selectividad del sistema de protección; pérdidas de elementos de red (líneas, transformadores) por sobrecargas transitorias producidas cuando el sistema trata de encontrar una nueva situación de equilibrio.

- Inestabilidad de ángulo: embalamiento o frenado de generadores por aislamiento del sistema, pérdida de sincronismo de un generador tras oscilación contra el resto del sistema, o entre áreas coherentes de generación.

- Inestabilidad de tensiones, que pueden llevar hasta una situación de colapso total.

- Pérdidas de generación y/o demanda asociadas a huecos de tensión (disminución transitoria del nivel de tensión en los nudos eléctricamente próximos a un cortocircuito a tierra, hasta que se produce la actuación de los sistemas de protección).

- Actuación de los relés de deslastre de carga por baja frecuencia. 
Capítulo 4. Características y efectos de la variabilidad de la potencia 96 eólica en España

Los huecos de tensión derivan de la perdida de la estabilidad de tensión en el sistema debido a una gran perturbación. Los huecos de tensión se han convertido en un problema muy extendido en los parques eólicos debido a la naturaleza asíncrona en la mayoría de estas instalaciones. Los aerogeneradores con una configuración en jaula de ardilla o DFIG (doblemente alimentado) de $1^{\mathrm{a}}$ generación no pueden funcionar de forma aislada y necesitan aportación de energía reactiva procedente de la red. Cuando se produce un hueco de tensión los generadores asíncronos son incapaces de continuar su funcionamiento y la producción se ve recortada.

La capacidad de asegurar la continuidad de suministro es una prioridad desde el punto de vista de la seguridad del sistema, y por tanto con implicaciones en el nivel de penetración permitido para la generación eólica en la red. Cuando se produce una falta en el sistema, la tensión cae a lo largo de toda la red provocando el disparo de los generadores que no tienen la capacidad de soportar la caída de tensión producida. Cuando la falta es despejada, el sistema necesita suficiente reserva de generación para cubrir la pérdida de generación durante el hueco. Durante las perturbaciones en la red, la estabilidad del sistema depende de los generadores conectados al sistema para restaurar la operación normal. La desconexión de generación durante una falta puede ocasionar problemas de calidad y en extremo, colapsos de tensión, derivados de una gran variación en la potencia generada por la producción eólica.

Desde la aparición del procedimiento de operación que regula la continuidad de suministro de parques eólicos ante huecos de tensión se han adaptado y certificado un gran número de parques eólico por lo que existe una gran cantidad de instalaciones, casi el $65 \%$ de la potencia instalada en Junio de 2009, que se consideran preparadas para soportar huecos de tensión muy severos sin que se produzca su desconexión de la red. El número de desconexiones mayores de $100 \mathrm{MW}$, y la potencia instalada que cumple en el procedimiento de operación 12.3 entre 2005 y 2010 se indica en la figura 4.1 .

Por otro lado, para la predicción eólica se ha empleado el modelo Sipreólico, descrito en el apartado 2.4.5.2. Normalmente la predicción se realiza de forma regional y se agrega para forma la predicción global.

A continuación se muestran las características de las fluctuaciones eólicas en el sistema eléctrico español.

\subsection{Características regionales de la variabilidad de la potencia eólica en España}

España es segundo país mas montañoso de Europa lo que junto con su extensión le da una considerable variedad climatológica. Esto conduce a que 
4.2. Características regionales de la variabilidad de la potencia eólica en

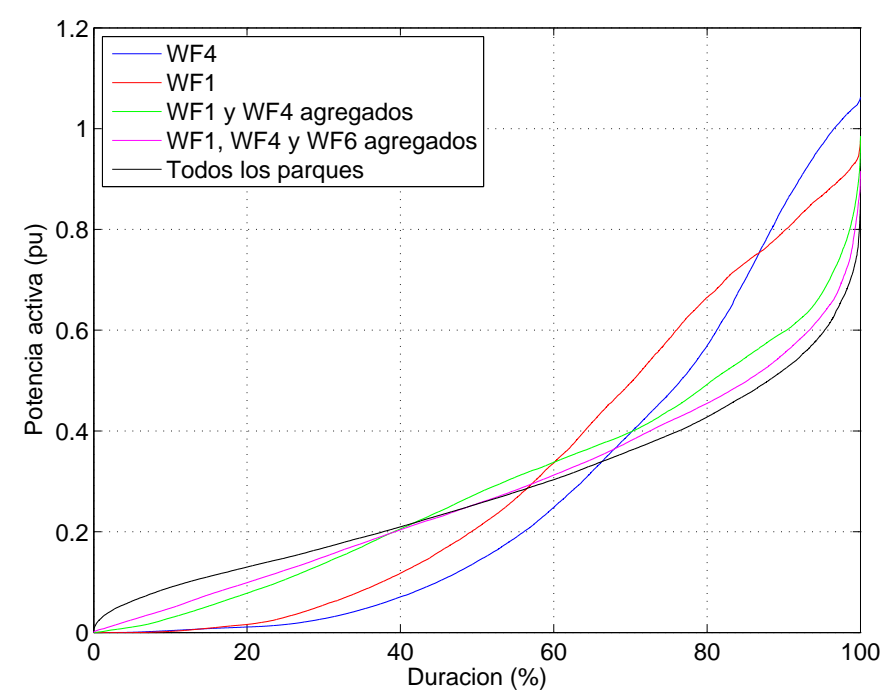

(a) 2007

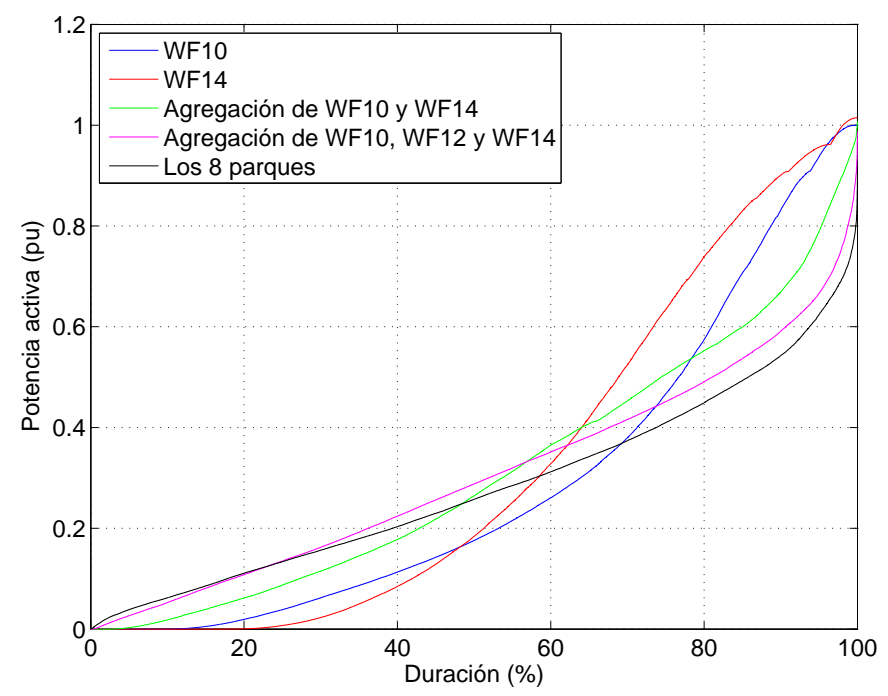

(b) 2008

Figura 4.2: Curvas de duración de parques y agregaciones consideradas.

las características de la velocidad de viento en distintas localizaciones sean muy dispares. Es por esto que el efecto de suavizado esta muy presente en las agregaciones de parques eólicos.

En la figura 4.2 se muestran curvas de duración para varios parques eólicos y agregaciones descritas en el capítulo 3. Se puede apreciar que las características de las curvas dependen del modelo de aerogenerador del parque y de las condiciones meteorológicas. Así se ve que el parque WF4 está formado por aerogeneradores con control aerodinámico pasivo, ya que alcan- 
Capítulo 4. Características y efectos de la variabilidad de la potencia 98 eólica en España

za valores de potencia por encima del 1 p.u., mientras que el parque WF1 incluye aerogeneradores con control de ángulo de pala ("pitch"). Así mismo el parque WF10 está ubicado en un emplazamiento con un menor recurso que el parque WF14 ya que la curva está por debajo en duraciones altas. Por otra parte, el nivel de agregación presente tiende a aplanar la curva de duración como ocurre en las agregaciones WF1+WF4, WF1+WF4+WF6, WF10+WF14 y WF10+WF12+WF14.

Las figuras 4.3 y 4.4 representan los valores de desviación estándar y PNRR-99 respectivamente para diversas agregaciones y para el total de producción española en los años 2007 y 2008. De este modo se puede apreciar el efecto de suavizado presente en cada agregación. También se distingue una leve dependencia del efecto de suavizado con la distancia ponderada y una gran dependencia del número de parques agregados.

\subsection{Características anuales de la variabilidad de la potencia eólica en España}

La evolución de la variabilidad de la potencia eólica en los últimos años en el sistema eléctrico español se muestra en la figura 4.5. En ella se muestra la desviación estándar de la generación eólica peninsular para cada mes desde el año 2007 hasta el año 2012. Se aprecia una clara influencia de la época del año en el parámetro, con una mayor variabilidad en los meses con mayor producción como son los meses de invierno y principio de primavera.

De forma similar, en la figura 4.6 se establece la desviación estándar y el PNRR-99 de las rampas. Existe una menor influencia del mes de año sobre estos parámetros que en la desviación estándar de la producción. En cambio ambos parámetros vienen marcados según la tendencia climatológica de ese año concreto, acentuandose ligeramente con una cierta tendencia en algunos meses. Adicionalmente, el valor de la desviación estándar está más influenciado por la temporalidad que el valor PNRR-99, el cual tiene mayor dispersión.

En la figura 4.7 se indican los valores medios de los datos anteriores estableciendo las condiciones de variabilidad típicas de cada mes. En el caso de la desviación estándar de las rampas los resultados muestran un máximo en el mes de Marzo y un mínimo en el mes de Septiembre.

\subsection{Rampas de potencia eólica extremas y sus causas en España}

En la tabla 4.1 se muestran los valores, la fecha y la causa principal de las rampas más extremas que han ocurrido en el sistema eléctrico español de 2007 a 2011 ambos inclusive para diferentes medias temporales. Se aprecia 


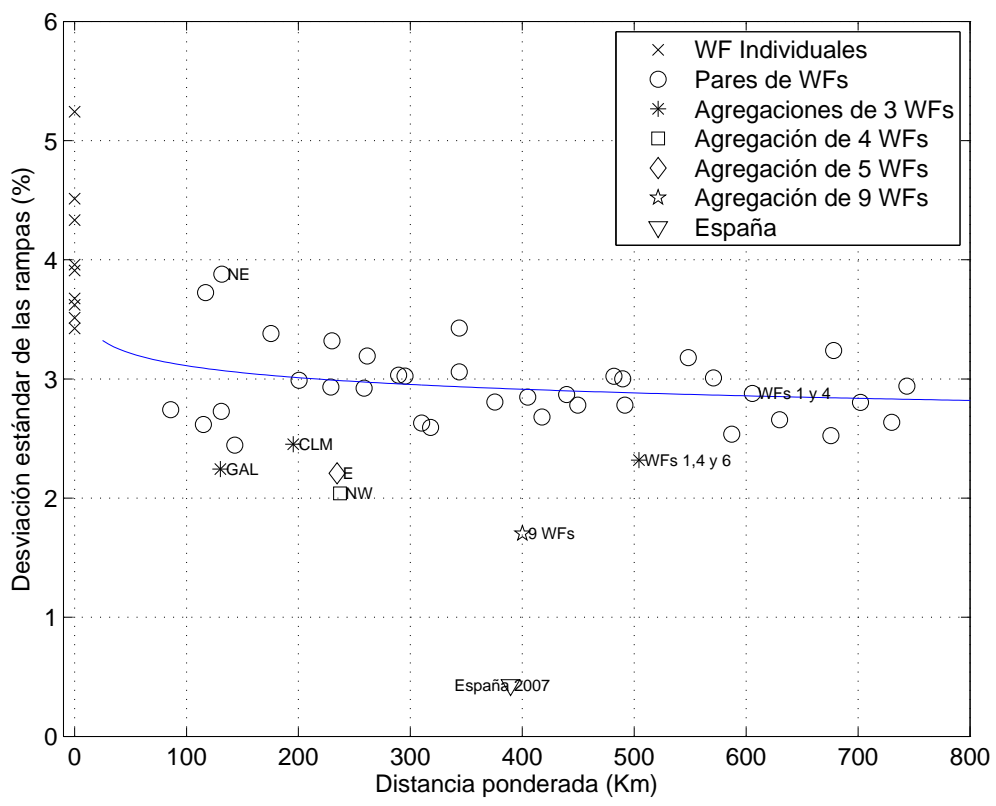

(a) 2007

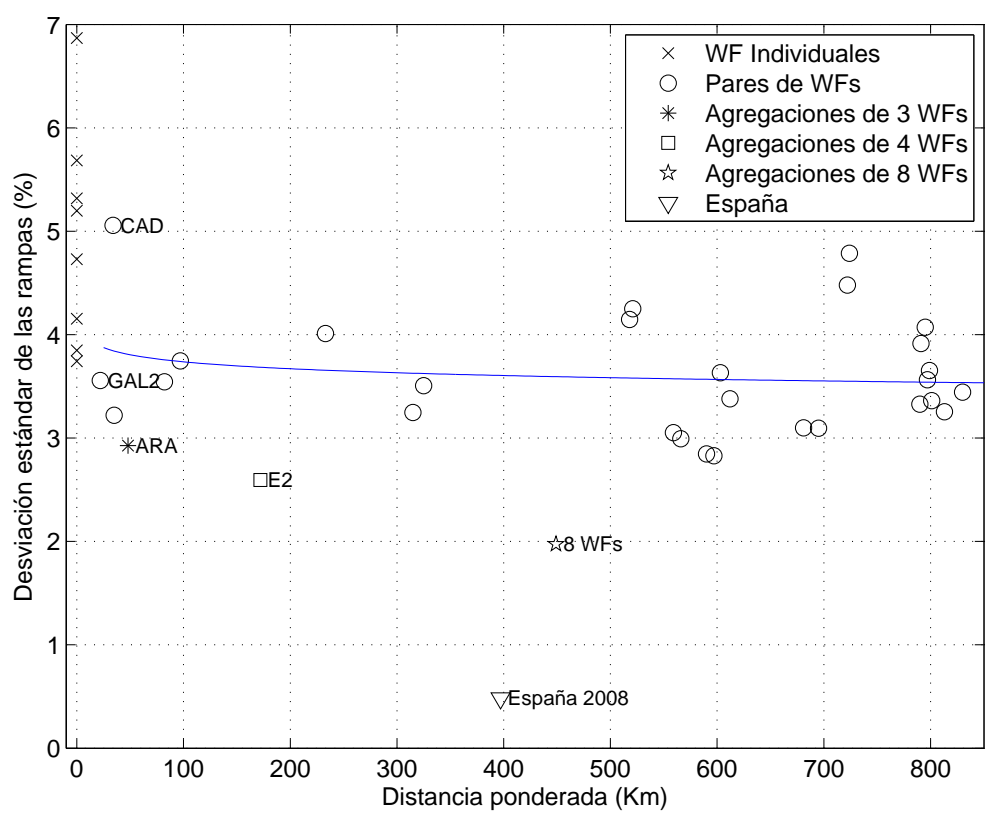

(b) 2008

Figura 4.3: Valores de desviación estándar para parques y agregaciones consideradas.

que para los datos de medias de 10 minutos las principales causas con los huecos de tensión y la variabilidad meteorológica del viento apenas provoca 
Capítulo 4. Características y efectos de la variabilidad de la potencia 100 eólica en España

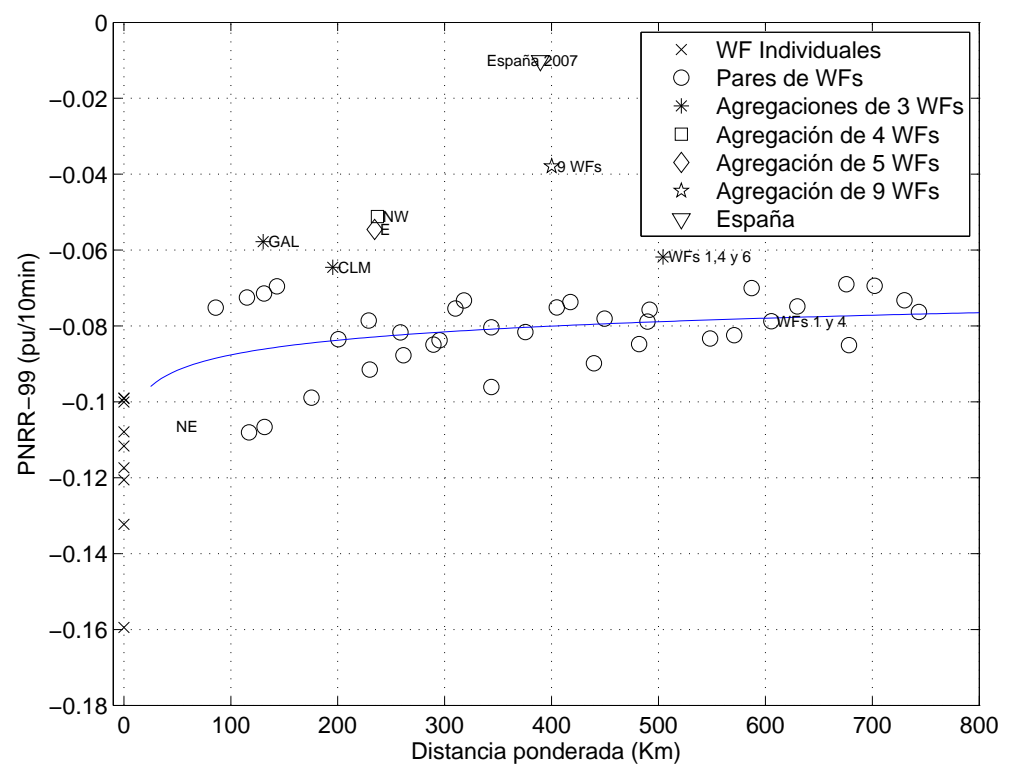

(a) 2007

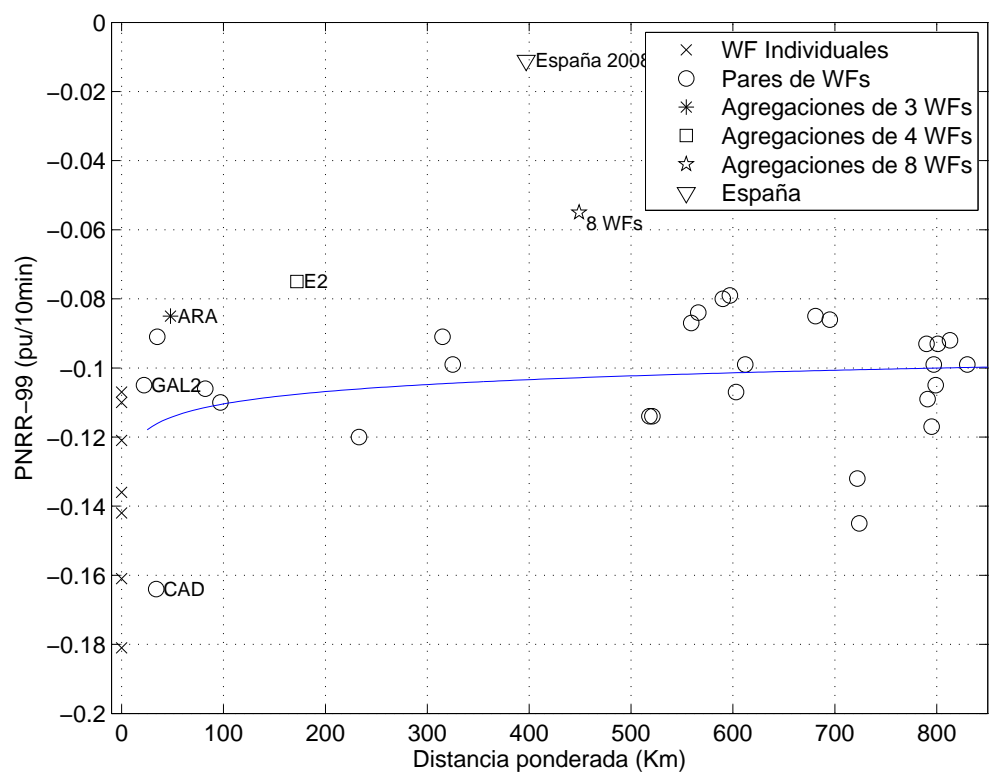

(b) 2008

Figura 4.4: Valores de PNRR99 para parques y agregaciones consideradas.

rampas extremas. En cambio cuando aumentamos el periodo de la media de los datos la variabilidad meteorológica del viento aparece como la principal causa. También hay que destacar la inclusión de rampas por recortes que son provocadas por el operador del sistema. Otro caso que provoca rampas 


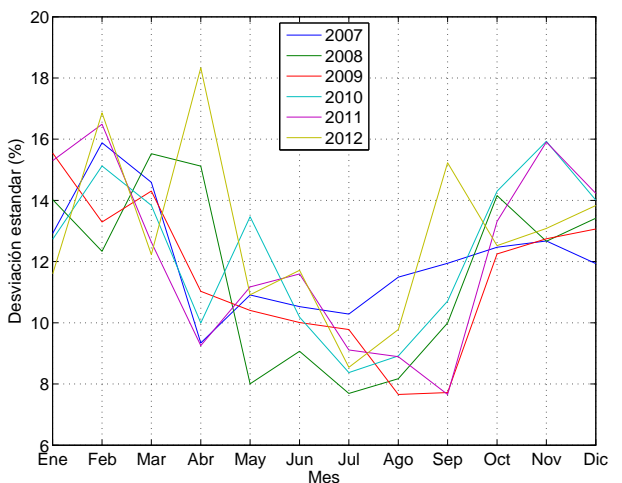

(a) Desviación estándar

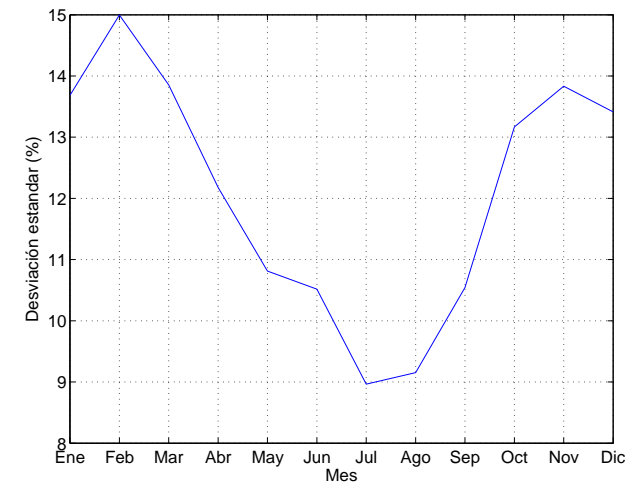

(b) Desviación estándar media

Figura 4.5: Desviación estándar de la potencia eólica en el sistema eléctrico español según meses del año

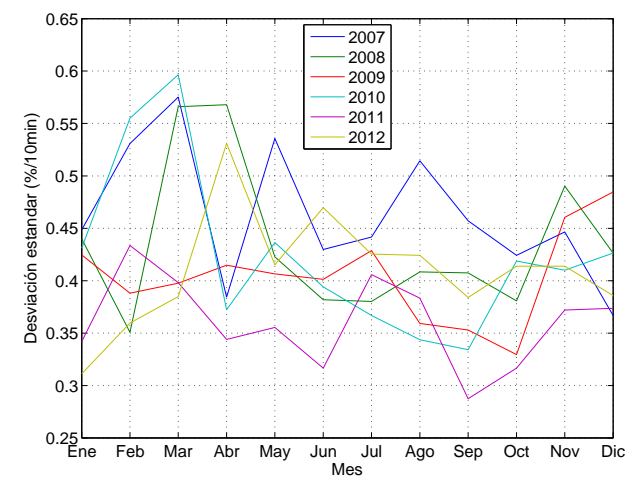

(a) Desviación estándar

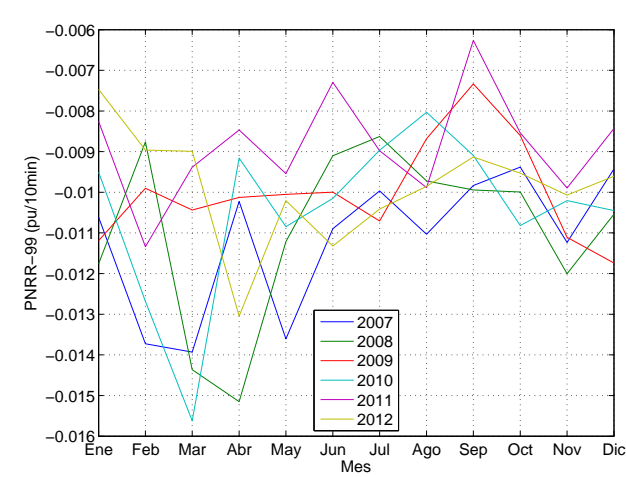

(b) PNRR-99

Figura 4.6: Desviación estándar de las rampas de la potencia eólica y PNRR99 en el sistema eléctrico español según meses del año

extremas es la protección por sobrevelocidad de los aerogeneradores, cuando vientos con una alta velocidad afectan a un area con una gran potencia instalada y se produce la desconexión de una gran cantidad de potencia eólica.

\subsection{Predicción de la energía eólica en el sistema eléctrico español}

En la figura 4.8 se indica la densidad de probabilidad de los errores de predicción de la potencia eólica del sistema eléctrico español en función de la potencia real. Los resultados se muestran para dos horizontes de predicción, 
Capítulo 4. Características y efectos de la variabilidad de la potencia eólica en España

\begin{tabular}{|c|c|c|c|c|c|c|}
\hline & \multicolumn{3}{|c|}{ Rampas negativas } & \multicolumn{3}{|c|}{ Rampas positivas } \\
\hline & Fecha & Valor & Causa principal & Fecha & Valor & Causa principal \\
\hline \multirow[t]{11}{*}{ Medias $10 \mathrm{~min}$} & $26 / 09 / 200716 \mathrm{~h}$ & -1143 & Hueco tensión & $01 / 03 / 201006 \mathrm{~h}$ & 2005 & Fin recorte \\
\hline & $17 / 03 / 201011 \mathrm{~h}$ & -1082 & Hueco tensión & $12 / 07 / 201123 \mathrm{~h}$ & 1419 & Hueco tensión \\
\hline & $21 / 07 / 200919 \mathrm{~h}$ & -990 & Hueco tensión & $02 / 11 / 200809 \mathrm{~h}$ & 1278 & Fin recorte \\
\hline & $19 / 03 / 200718 \mathrm{~h}$ & -989 & Hueco tensión & $26 / 09 / 200717 \mathrm{~h}$ & 1233 & Hueco tensión \\
\hline & $20 / 03 / 200700 \mathrm{~h}$ & -966 & Hueco tensión & $17 / 03 / 201011 \mathrm{~h}$ & 1216 & Hueco tensión \\
\hline & $26 / 01 / 200917 \mathrm{~h}$ & -937 & Hueco tensión & $24 / 02 / 201006 \mathrm{~h}$ & 1114 & Fin recorte \\
\hline & $26 / 02 / 201000 \mathrm{~h}$ & -931 & Principio recorte & $01 / 03 / 201006 \mathrm{~h}$ & 1029 & Fin recorte \\
\hline & $02 / 03 / 201014 \mathrm{~h}$ & -931 & Principio recorte & $30 / 07 / 200716 \mathrm{~h}$ & 1019 & Hueco tensión \\
\hline & $24 / 02 / 201001 \mathrm{~h}$ & -927 & Principio recorte & $27 / 02 / 201019 \mathrm{~h}$ & 935 & Variab. viento \\
\hline & $01 / 01 / 201001 \mathrm{~h}$ & -911 & Principio recorte & $26 / 02 / 201006 \mathrm{~h}$ & 932 & Fin recorte \\
\hline & Fecha & Valor & Causa principal & Fecha & Valor & Causa principal \\
\hline \multirow[t]{13}{*}{ Media 1 hora } & $26 / 02 / 20100: 59$ & -1957 & Principio recorte & $02 / 11 / 20088: 59$ & 2077 & Velocidad de corte \\
\hline & $01 / 01 / 201001 \mathrm{~h}$ & -1844 & Principio recorte & $01 / 03 / 201006 \mathrm{~h}$ & 2023 & Fin recorte \\
\hline & $25 / 02 / 201000 \mathrm{~h}$ & -1837 & Principio recorte & $01 / 03 / 201005 \mathrm{~h}$ & 1951 & Fin recorte \\
\hline & $08 / 03 / 201001 \mathrm{~h}$ & -1722 & Principio recorte & $14 / 02 / 201113 \mathrm{~h}$ & 1723 & Variab. viento \\
\hline & $01 / 03 / 201001 \mathrm{~h}$ & -1541 & Principio recorte & $23 / 12 / 200907 \mathrm{~h}$ & 1644 & Variab. viento \\
\hline & $26 / 08 / 201118 \mathrm{~h}$ & -1539 & Variab. viento & $25 / 08 / 201112 \mathrm{~h}$ & 1615 & Variab. viento \\
\hline & $28 / 02 / 201118 \mathrm{~h}$ & -1535 & Variab. viento & $20 / 02 / 201113 \mathrm{~h}$ & 1574 & Variab. viento \\
\hline & $23 / 12 / 200902 \mathrm{~h}$ & -1491 & Principio recorte & $23 / 12 / 201117 \mathrm{~h}$ & 1569 & Variab. viento \\
\hline & $22 / 08 / 201101 \mathrm{~h}$ & -1451 & Principio recorte & $25 / 08 / 201113 \mathrm{~h}$ & 1530 & Variab. viento \\
\hline & $25 / 02 / 201002 \mathrm{~h}$ & -1450 & Principio recorte & $12 / 07 / 201112 \mathrm{~h}$ & 1513 & Variab. viento \\
\hline & 06/03/2010 01h & -1449 & Principio recorte & $02 / 11 / 200805 \mathrm{~h}$ & 1475 & Velocidad de corte \\
\hline & $24 / 02 / 201001 \mathrm{~h}$ & -1429 & Principio recorte & $23 / 12 / 201116 \mathrm{~h}$ & 1472 & Variab. viento \\
\hline & Fecha & Valor & Causa principal & Fecha & Valor & Causa principal \\
\hline \multirow[t]{12}{*}{ Media 4 horas } & $31 / 12 / 200921 \mathrm{~h}$ & -3454 & Principio recorte & $25 / 08 / 201105 \mathrm{~h}$ & 3970 & Variab. viento \\
\hline & $15 / 02 / 201101 \mathrm{~h}$ & -3412 & Variab. viento & $01 / 03 / 201001 \mathrm{~h}$ & 3934 & Fin recorte \\
\hline & $26 / 08 / 201113 \mathrm{~h}$ & -3370 & Variab. viento & $12 / 07 / 201105 \mathrm{~h}$ & 3916 & Variab. viento \\
\hline & $01 / 03 / 201009 \mathrm{~h}$ & -3283 & Principio recorte & $25 / 08 / 201109 \mathrm{~h}$ & 3857 & Variab. viento \\
\hline & $13 / 02 / 201113 \mathrm{~h}$ & -3261 & Variab. viento & $15 / 11 / 200901 \mathrm{~h}$ & 3610 & Variab. viento \\
\hline & $26 / 12 / 201005 \mathrm{~h}$ & -3045 & Variab. viento & $03 / 05 / 201005 \mathrm{~h}$ & 3584 & Variab. viento \\
\hline & $23 / 01 / 200913 \mathrm{~h}$ & -2896 & Variab. viento & $23 / 12 / 200901 \mathrm{~h}$ & 3579 & Fin recorte \\
\hline & $25 / 10 / 201109 \mathrm{~h}$ & -2869 & Variab. viento & $25 / 12 / 200913 \mathrm{~h}$ & 3456 & Variab. viento \\
\hline & $02 / 03 / 201021 \mathrm{~h}$ & -2849 & Principio recorte & $23 / 12 / 201109 \mathrm{~h}$ & 3401 & Variab. viento \\
\hline & $17 / 02 / 201021 \mathrm{~h}$ & -2841 & Variab. viento & $13 / 09 / 201109 \mathrm{~h}$ & 3323 & Variab. viento \\
\hline & $21 / 07 / 200913 \mathrm{~h}$ & -2820 & Variab. viento & $07 / 09 / 201005 \mathrm{~h}$ & 3198 & Variab. viento \\
\hline & $04 / 04 / 200721 \mathrm{~h}$ & -2799 & Variab. viento & $04 / 02 / 201009 \mathrm{~h}$ & 3181 & Variab. viento \\
\hline
\end{tabular}

Tabla 4.1: Enumeración de las rampas eólicas más extremas acaecidas en el sistema eléctrico español entre 2007 y 2011 ambos inclusive y sus principales causas 


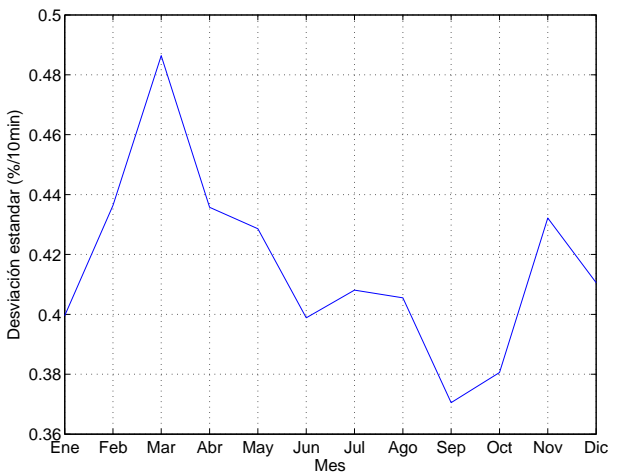

(a) Desviación estándar media

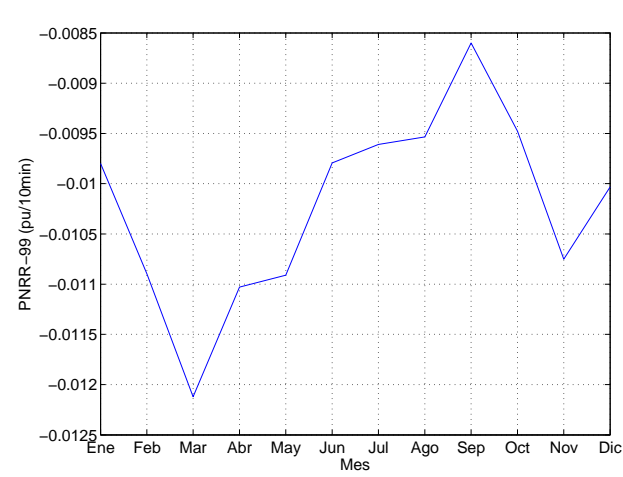

(b) PNRR-99 medio

Figura 4.7: Parámetros medios de la desviación estándar de las rampas de la potencia eólica y PNRR-99 en el sistema eléctrico español según meses del año

1 hora, figura 4.8a, y 24 horas, figura 4.8b. Se aprecia que la dispersión aumenta al aumentar la potencia hasta los $12000 \mathrm{MW}$ a partir de cuando la dispersión decrece. La media de los errores es mas pronunciada con el horizonte de predicción de 24 horas y a partir de los 2000 MW es negativa.

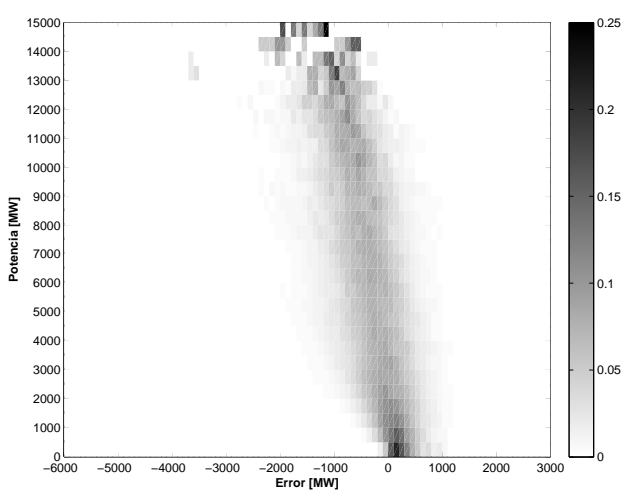

(a) 1 hora

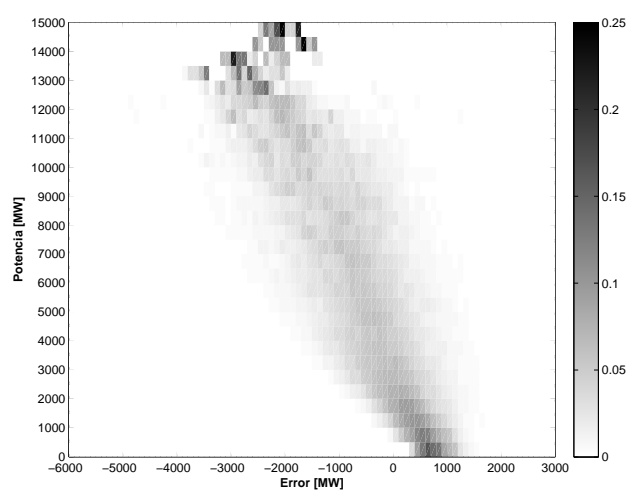

(b) 24 horas

Figura 4.8: Densidad de probabilidad del error de predicción eólica en función de la potencia real entre 2007 y 2011

Los datos obtenidos en la figura 4.8 son ajustados tanto en media como en desviación estándar a polinomios de diverso orden obteniendo lo mostrado en la figura 4.9 tanto para la media como para la desviación estándar.

Los polinomios resultantes son, para el error medio: 
CApítulo 4. Características y efectos de la variabilidad de la potencia eólica en España

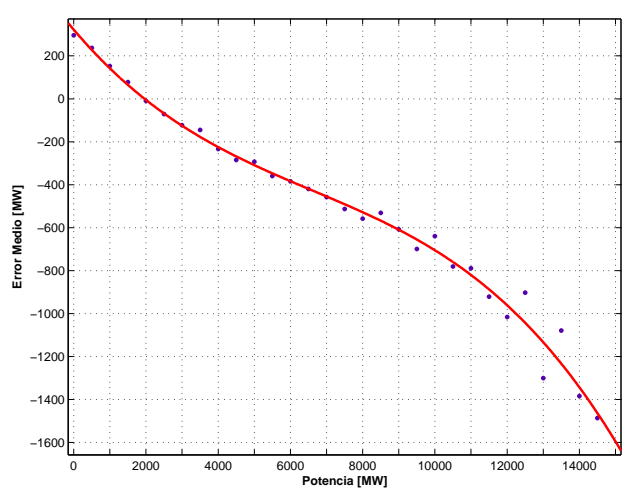

(a) Media

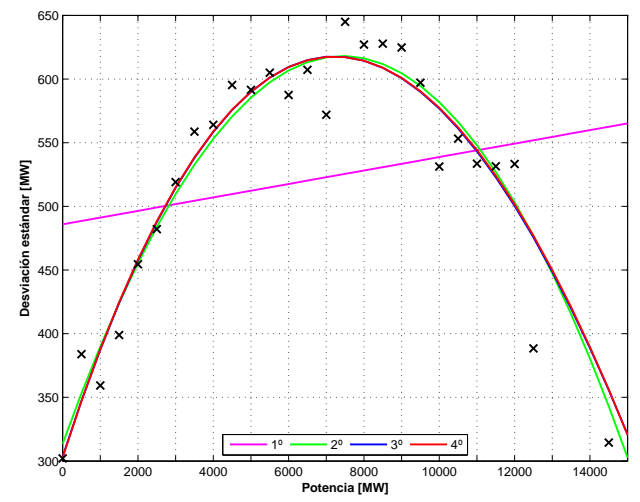

(b) Desviación estándar

Figura 4.9: Ajuste de la media aritmética y la desviación estándar del error de predicción eólica en función de la potencia real a curvas polinómicas de primer, segundo, tercer y cuarto orden

$P_{\text {emedio }}(x)=-2,616 e-014 \cdot x^{4}-2,133 e-010 \cdot x^{3}+1,228 e-005 \cdot x^{2}-0,1766 x+308,9$

y para la desviación estándar:

$$
P_{\text {estd } 1}(x)=0,005281 \cdot x+485,9
$$

$$
P_{e s t d 2}(x)=-5,51 e-06 \cdot x^{2}+0,08193 \cdot x+313,5
$$

$$
P_{\text {estd } 3}(x)=7,58 e-11 \cdot x^{3}-7,13 e-06 \cdot x^{2}+0,09105 \cdot x+303,5
$$

$P_{\text {estd } 4}(x)=-1,56 e-15 \cdot x^{4}+1,21 e-10 \cdot x^{3}-7,54 e-06 \cdot x^{2}+0,09232 \cdot x+302,8$

El polinomio de orden dos, ecuacion 4.5, se aproxima con bastante precisión a los puntos calculados.

Del mismo modo en la figura 4.10 se obtienen los ajustes para la media y la desviación estándar pero en rangos de 6 horas. Se aprecian resultados similares a los obtenidos anteriormente sin diferencias significativas entre los rangos de 6 horas. 


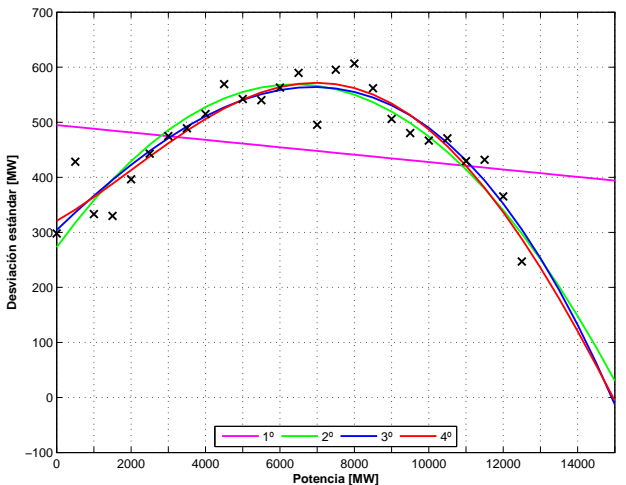

(a) de 0 a $6 \mathrm{~h}$

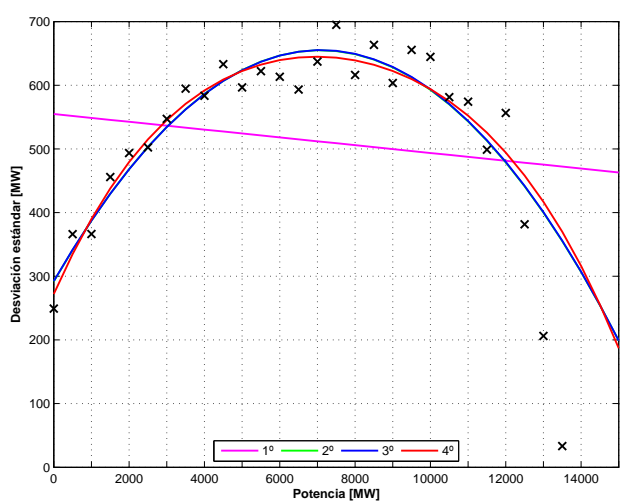

(c) de 12 a $18 \mathrm{~h}$

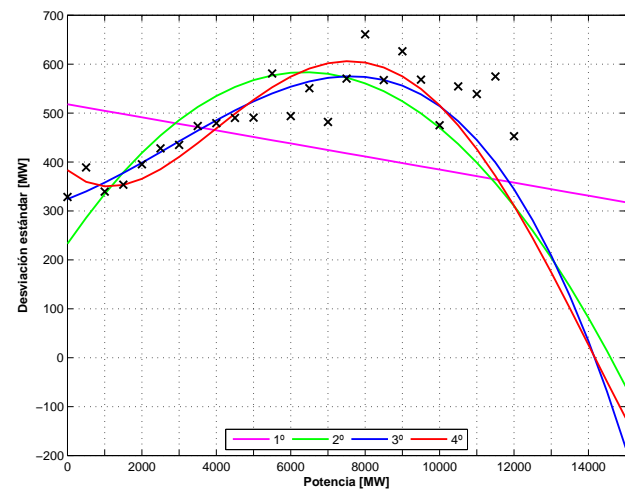

(b) de 6 a $12 \mathrm{~h}$

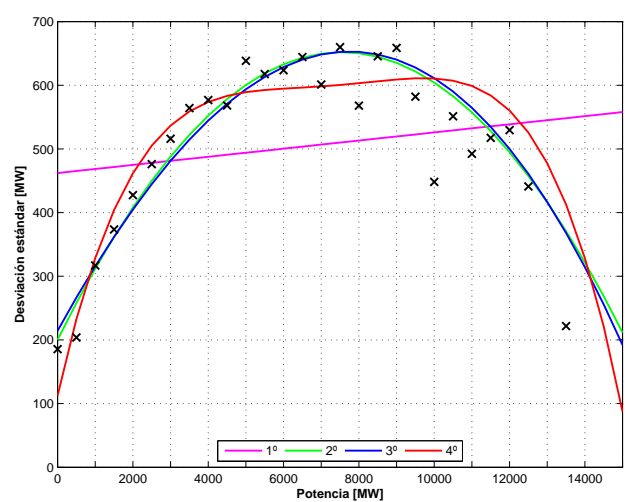

(d) de 18 a $24 \mathrm{~h}$

Figura 4.10: Ajuste de la desviación estándar del error de predicción eólica por rangos de horas en función de la potencia real a curvas polinómicas de primer, segundo, tercer y cuarto orden

\subsection{Evolución de las reservas en el sistema eléctrico español}

En la figura 4.11 se muestra la evolución de las reservas secundarias y terciarias, figura $4.11 \mathrm{~b}$, y totales (suma de secundarias y terciarias), figura 4.11a en los últimos años. Se aprecia que en 2009 y 2010 la regulación a bajar subió considerablemente. Estos años, especialmente 2009 fueron años húmedos, reservas hidraulicas saturadas y con una gran producción eólica.

\subsection{Ejemplos de eventos extremos}

En esta sección se analizan varios ejemplos de eventos extremos ocurridos en la red eléctrica española en los últimos años. Estos eventos se han clasifi- 
Capítulo 4. Características y efectos de la variabilidad de la potencia 106 eólica en España

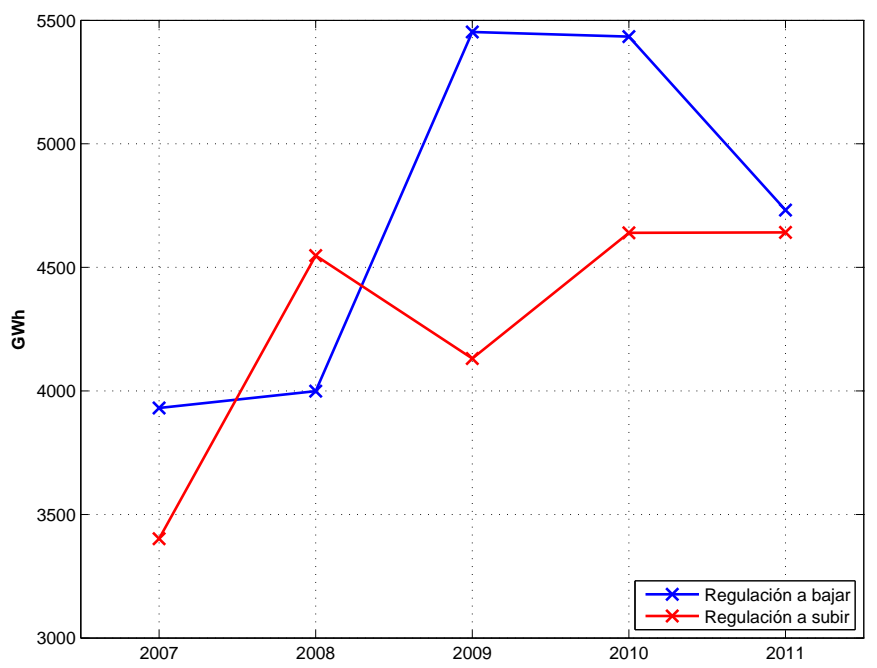

(a) Reserva total a bajar y a subir

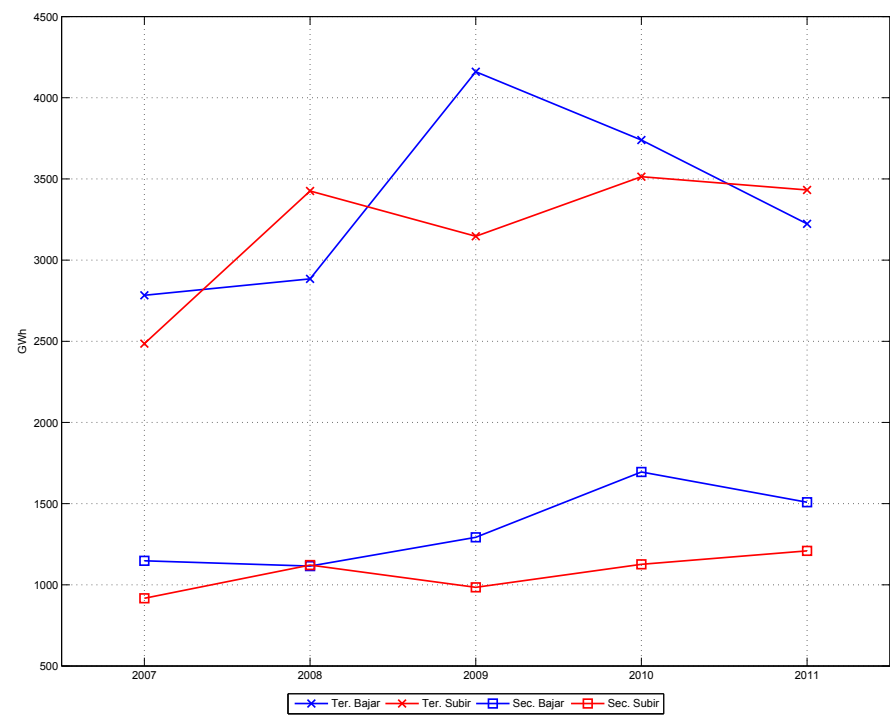

(b) Reservas secundarias y terciarias

Figura 4.11: Comparación de las reservas de regulación desde 2007 a 2011.

cado en función de su origen tal y como se describen en Martín-Martínez et al. (2012).

\subsubsection{Fenómenos meteorológicos. Tormentas, ciclones y cal- mas}

En este apartado se incluyen ejemplos de eventos en los que las condiciones de viento han sido extremas, tanto por exceso como por defecto o 
porque las predicciones han incluido grandes errores y distan considerablemente de la producción real.

\subsubsection{Tormenta Klaus. 23 y 24 de enero de 2009}

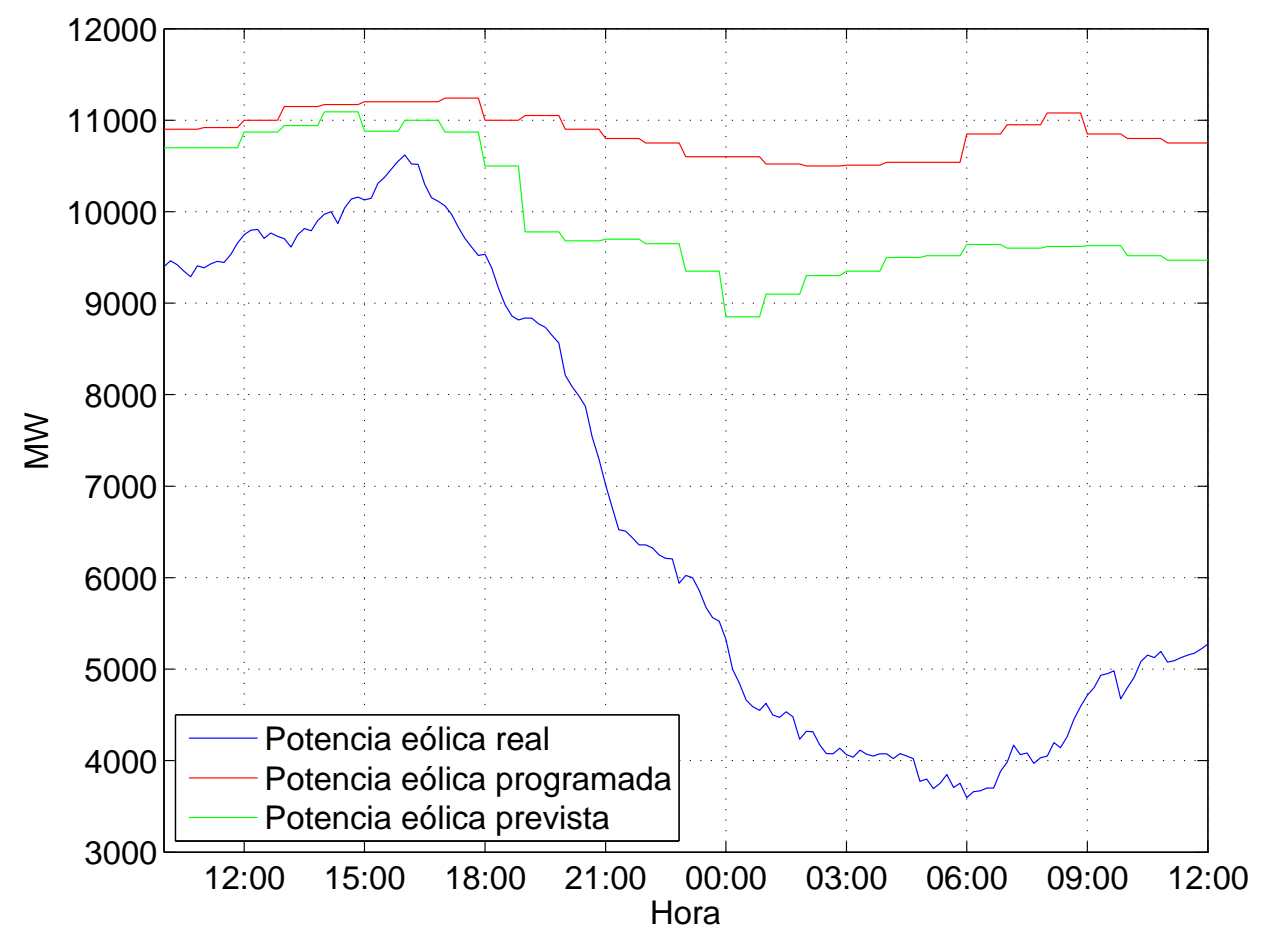

Figura 4.12: Producción eólica, predicción eólica y generación eólica programada durante la tormenta Klaus

Como ejemplo cabe destacar la tormenta Klaus. El ciclón extratropical Klaus fue un ciclón de latitud media que afectó los días 23, 24 y 25 de enero de 2009 especialmente al norte de España y sur de Francia ocasionando varios muertos e importantes daños materiales. El centro de la depresión se desplazó de oeste a este desde las Islas Azores, pasando por el Mar Cantábrico, hasta el golfo de Génova; generando fuertes vientos de más de 150 $\mathrm{km} / \mathrm{h}$ en el litoral español y francés. Esto produjo la desconexión de muchos parques en las regiones septentrionales de España. Además, tanto las instalaciones eólicas como las grandes redes de transporte y distribución eléctrica se vieron seriamente afectadas a lo largo de esas provincias, registrándose diversos daños en aerogeneradores y la caída de varios tramos de apoyos en líneas de alta tensión. El resultado final conllevó a la desconexión de 7000 MW por protección por sobrevelocidad. Por otra parte también se redujo la demanda, con una reducción adicional de la misma al dañarse algunas líneas, 
CAPítulo 4. Características y efectos de la variabilidad de la potencia 108 eólica en España

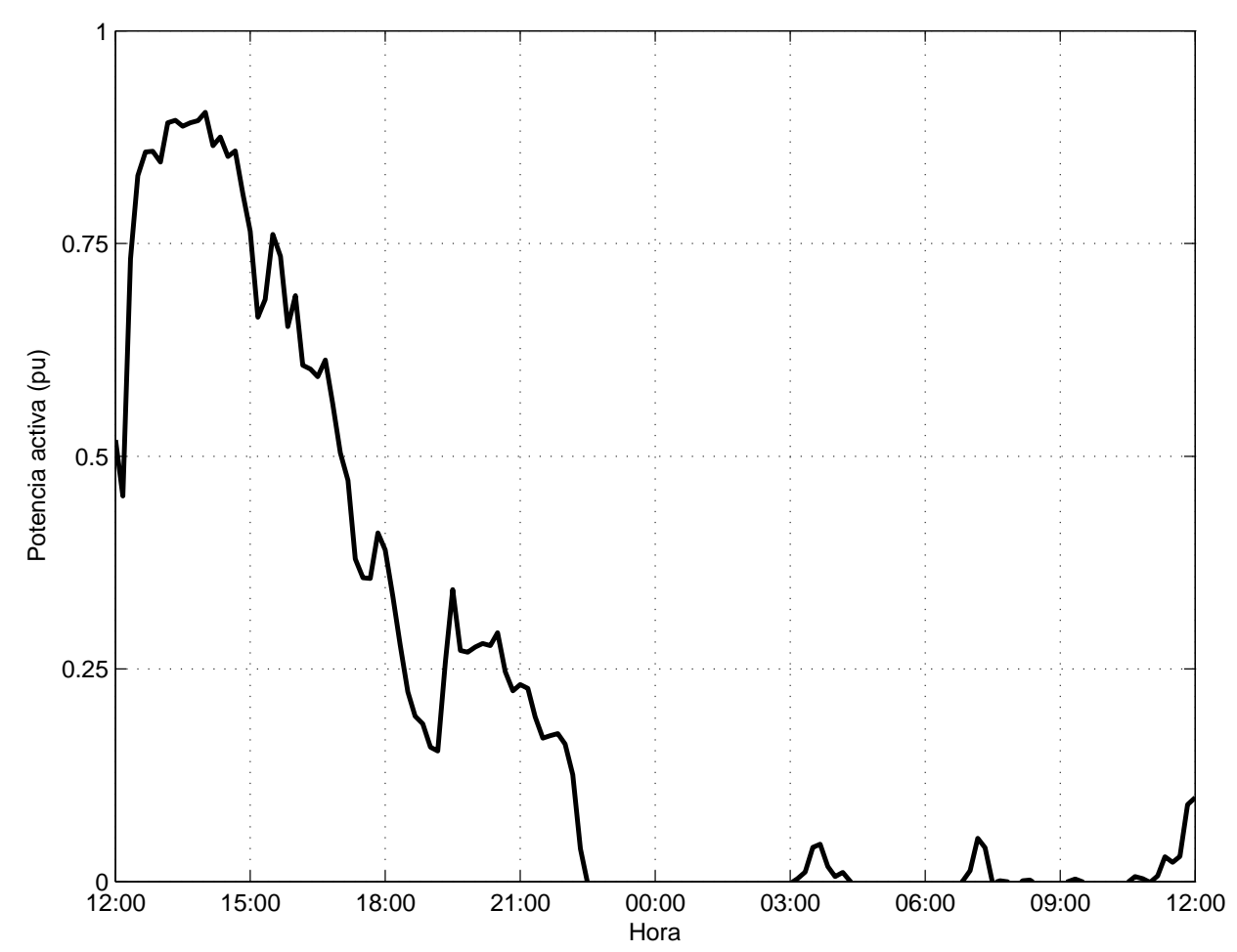

Figura 4.13: Producción eólica del parque WF13 de 49.5 MW durante la tormenta Klaus

y, afortunadamente, el recorte eólico coincidió con la bajada nocturna de la demanda. Todo esto se puede apreciar en la figura 4.12.

El desarrollo del evento ocurrió como se relata a continuación. Se esperaban fuertes vientos en la vertiente atlántica a partir de la tormenta Klaus, con una predicción de aproximadamente $11 \mathrm{GW}$ de potencia para la mayoría del día 23 y la noche del 23 al 24 . Por el contrario la velocidad de viento fue inesperadamente baja la mañana del día 23 y la potencia solo alcanzó, aproximadamente, los 9500 MW. Después la velocidad comenzó a subir registrando un pico sobre las 16:00. A partir de ese momento la velocidad de viento fue superior a la velocidad de corte de muchos aerogeneradores, desconectándose una gran cantidad de potencia en el norte del país, por la protección por sobre velocidad de los mismos. Esta caída repentina en la producción no estaba contemplada en la predicción. A las 21:00 h la generación eólica había caído sobre 7000 MW, unos 4000 MW por debajo de la predicción para esa hora. La situación empeoró al alcanzarse un error de 7000 MW a las 06:30 del día 24.

Este evento fue inusual por dos razones. Por una parte, los vientos que se produjeron fueron realmente extremos y alguno parques registraron veloci- 
dades de viento de hasta $220 \mathrm{~km} / \mathrm{h}$. Por otra parte, la severidad del evento no estaba reflejada en la predicción. La red esperaba altas velocidades de viento pero no lo suficientemente grandes como para conllevar la desconexión de los aerogeneradores. Estas dos razones provocaron numerosos problemas en la red de transporte y de distribución.

El centro de control (CECOEL) aumentó el nivel de generación hidráulica presente, incluyendo las centrales de bombeo, y de forma temporal, importó potencia a través de las conexiones internacionales. Una vez realizados estos pasos el sistema volvió al equilibrio y empezó a exportar a partir de las 22:00 del día 23. Las centrales de carbón y de ciclo combinado funcionaron a carga nominal has las 23:00 del día 23 y fueron reduciendo carga una vez que la demanda fue bajando durante la noche.

Este evento puede apreciarse desde el punto de vista del parque eólico WF13 en la figura 4.13. Se ve claramente cuando comienza a actuar la protección por sobrevelocidad de los aerogeneradores y la producción se viene abajo.

\subsubsection{Periodo de calma. 4 de febrero de 2007 .}

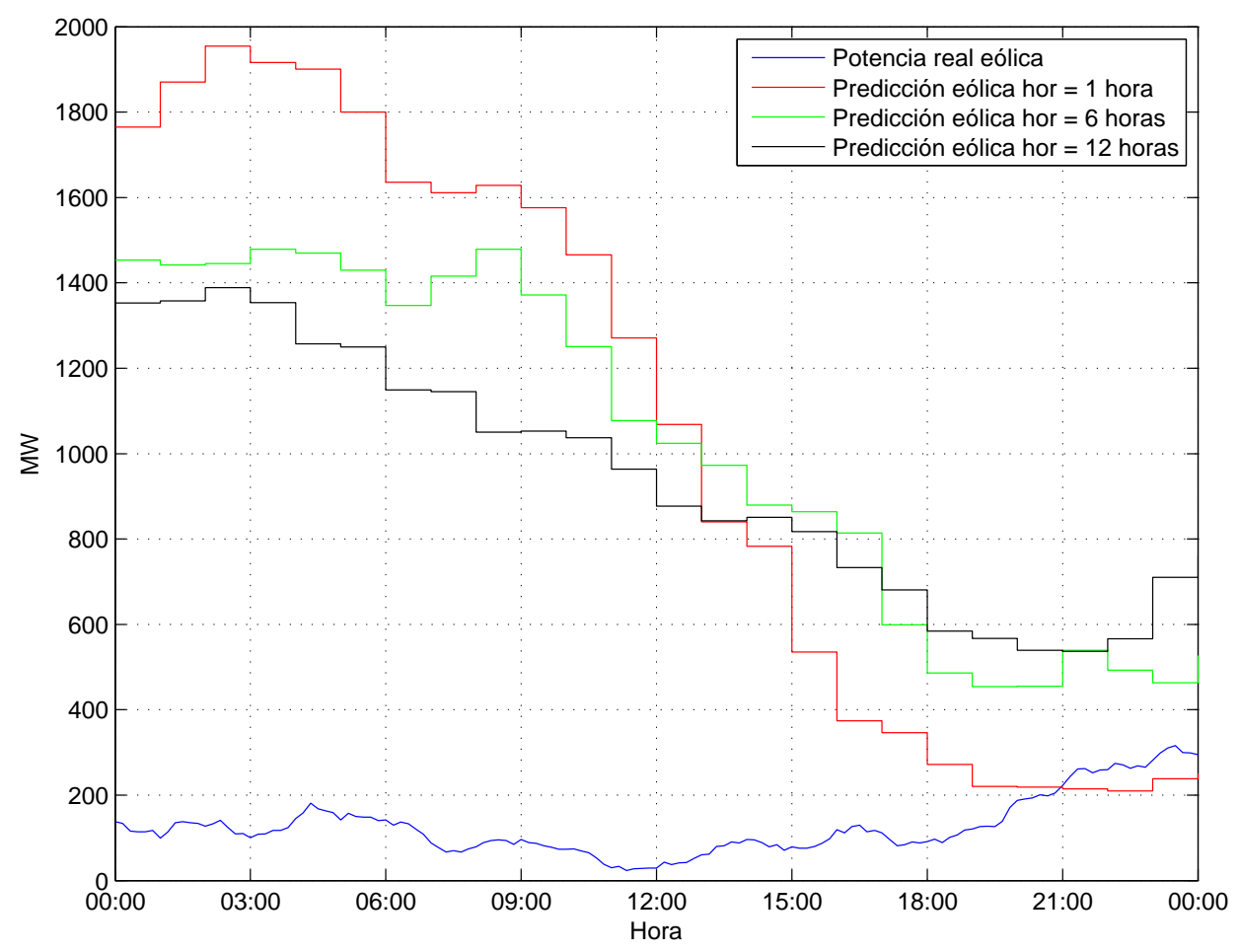

Figura 4.14: Producción eólica y predicción eólica con varios horizontes de predicción para el periodo de calma del 4 de febrero de 2007 
Capítulo 4. Características y efectos de la variabilidad de la potencia 110 eólica en España

La figura 4.14 muestra la producción eólica y su predicción para diferentes horizontes de predicción con un periodo de una hora durante el día 4 de febrero de 2007. Se muestra el mínimo registrado por la producción eólica de apenas $52 \mathrm{MW}$ esto supone un $0.3 \%$ de la potencia instalada y es considerado un periodo de calma total. Así mismo, se observa un error considerable entre las diferentes predicciones y la producción real.

\subsubsection{Recortes por saturación de la generación}

Este tipo de "fluctuaciones" vienen dadas por orden del operador del sistema ante la incertidumbre sobre el mantenimiento de la estabilidad del sistema cuando la demanda es muy baja, la generación eólica muy alta y la rigidez del sistema eléctrico restante hace inviable integrar toda la generación disponible.

Como ejemplo de esta situación tenemos la madrugada del 24 de febrero de 2010, cuando con una producción de aproximadamente $11800 \mathrm{MW}$, REE dio la orden de reducir la generación eólica en 800 MW, después de desacoplar grupos térmicos. La causa de esta reducción es la establecida en el apartado 4 del P.O. 3.7 como "Excedentes de generación no integrables en el sistema". Estos recortes tienen una pronunciada incidencia sobre el sistema, en relación con la pérdida de ingresos, el impacto en la operación de los parques y la respuesta mecánica de las maquinas, y la incidencia en la operación de la generación.

\subsubsection{Sobrerespuesta 1 de enero de 2010 .}

En la figura 4.15, se representa un caso de sobrerespuesta al recorte durante el día 1 de enero de 2010. El operador del sistema ordeno unas consignas de generación desde las 03:00 a las 07:00. Durante ese periodo de tiempo la velocidad de viento sobrepaso la velocidad de corte de los aerogeneradores en múltiples emplazamientos, por lo que la generación cayó muy por debajo de lo previsto. Esto supuso la entrada de reservas a subir para suplir esa pérdida inesperada.

En el caso del parque eólico WF13, la evolución del evento se muestra en la figura 4.16. La secuencia de las consignas es la siguiente:

- Desde las 00:00 a las 03:00, no existe ningún recorte. La mayoría de los aerogeneradores se encuentran a alta carga. Sobre las 02:10 hay una pequeña caída producida por un descenso de la velocidad de viento.

- Desde las 03:00 a las 04:40, se manda y se aplica una consigna de $0.6 \mathrm{pu}$ desde el operador del sistema. A la vez, una cantidad considerable de aerogeneradores se desconectan por protección contra sobrevelocidad y la producción baja hasta $0.1 \mathrm{pu}$, muy por debajo de la consigna. Des- 


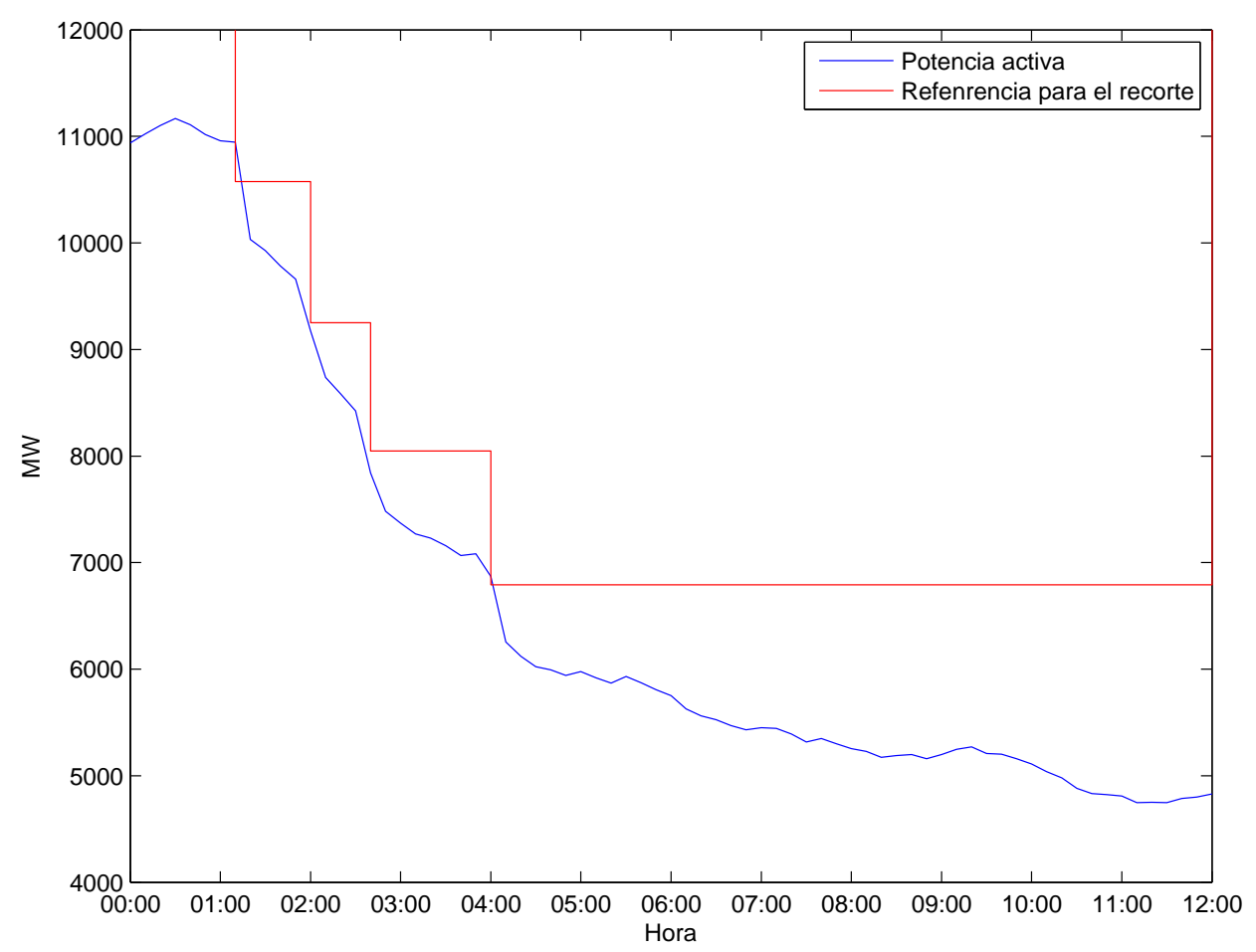

Figura 4.15: Producción eólica durante la sobrerespuesta del 1 de enero de 2010

pués muchos de los aerogeneradores se volvieron a conectar alcanzando así casi la consigna señalada.

- Desde las 04:40 a las 07:40, el operador del sistema cambió la consigna de 0.6 pu a 0.5 pu. De esta forma más aerogeneradores fueron desconectados.

- Finalmente, a las 07:40, el operador elimina la consigna y el parque pasa a funcionar a la máxima potencia disponible.

Los valores alcanzados durante este recorte se resumen en la tabla 4.2.

Las principales causas de la sobrerespuesta son:

- Los recortes se realizan durante periodos con una baja demanda y una gran producción eólica.

- Durante estos periodos, los parques eólicos operan a altas velocidades de viento y por lo tanto la fluctuaciones suelen ser bastante importantes. Se pueden producir periodos en los que se active la protección por sobrevelocidad. 
Capítulo 4. Características y efectos de la variabilidad de la potencia 112 eólica en España

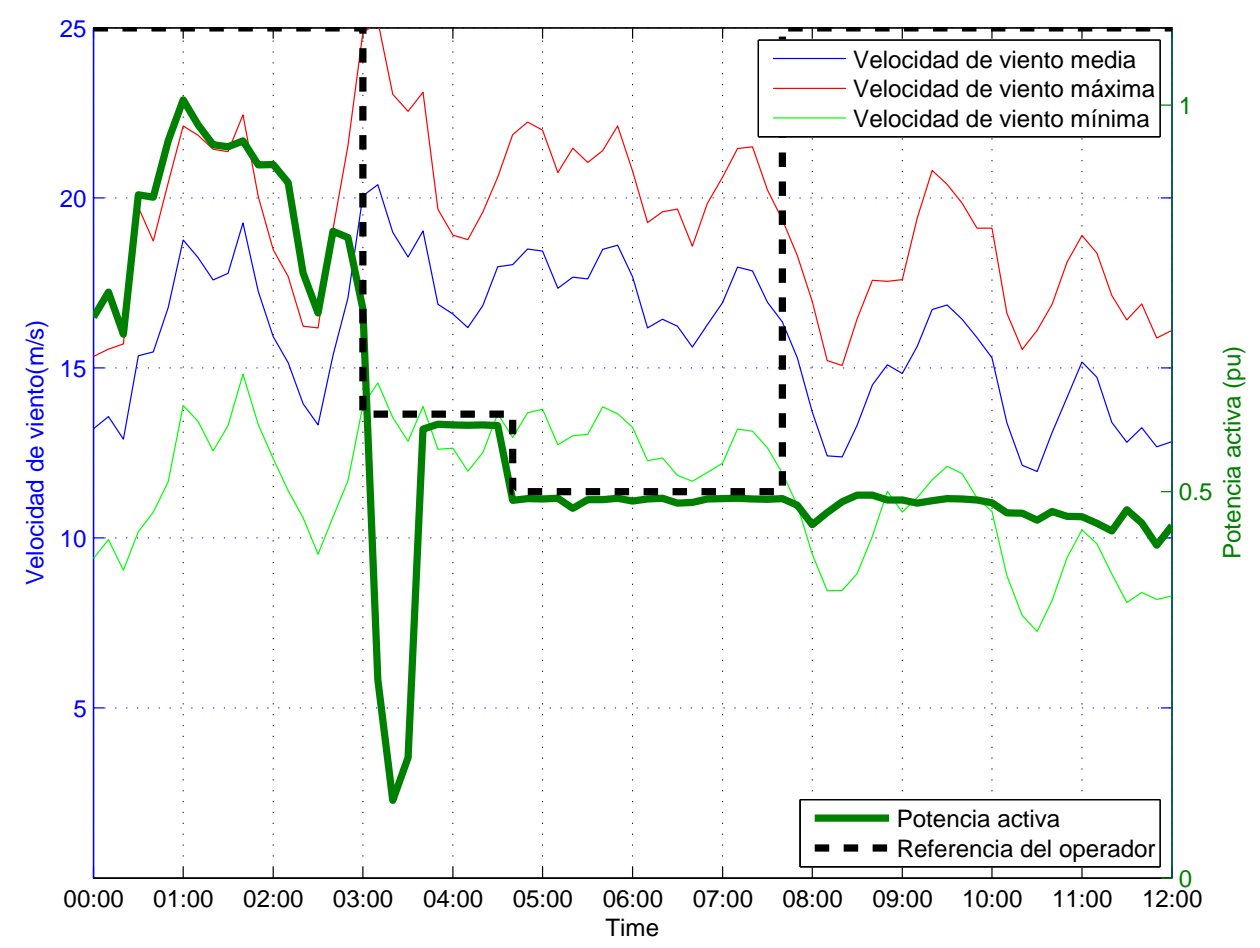

Figura 4.16: Producción eólica y velocidad de viento en el parque WF13 de 49.5 MW durante la sobrerespuesta del 1 de enero de 2010

- Los recortes se aplican desconectando una cierta cantidad de aerogeneradores en lugar de bajar carga en todos ellos. El número de aerogeneradores desconectados depende de la consigna y de la carga actual de los aerogeneradores.

\subsubsection{Perturbaciones de la red. Huecos de tensión y faltas}

En este apartado se muestran 3 ejemplos de variaciones de potencia eólica debidos a huecos de tensión.

\subsubsection{Hueco de tensión. 30 de marzo de 2009 .}

El 30 de marzo de 2009 a las 22:00 una falta se registró en la subestación de Barcina, resultando un hueco de tensión y produciendo una pérdida instantánea de 518 MW de potencia eólica. Para compensar esta caída, se produjo un desvió de la conexión UCTE de 826 MW para mantener el balance. Esta situación fue corregida con generación convencional a los 4 minutos. En la figura 4.17 se muestra la potencia eólica y el intercambio UCTE durante el hueco. 


\begin{tabular}{rrrrrr} 
Comienzo & Fin & Potencia (MW) & Referencia (MW) & Cambio real (MW) & Ratio \\
\hline $1: 10$ & $2: 00$ & 10944 & 10576 & -957 & 2.60 \\
$2: 00$ & $2: 35$ & 9642 & 9252 & -958 & 2.46 \\
$2: 35$ & $4: 00$ & 8445 & 8047 & -961 & 2.42 \\
$4: 00$ & $12: 00$ & 6981 & 6792 & -760 & 4.02 \\
$12: 00$ & $12: 05$ & 4830 & Sin consigna & - & - \\
$12: 05$ & $12: 37$ & 5633 & 6461 & -474 & -0.57 \\
$12: 37$ & $13: 12$ & 5190 & 6700 & 168 & 0.11 \\
$13: 12$ & $17: 40$ & 5374 & 7000 & 110 & 0.07 \\
$17: 40$ & - & 4645 & Sin consigna & - & - \\
\hline
\end{tabular}

Tabla 4.2: Sobrerespuesta del recorte del 1 de Enero de 2010

\subsubsection{Hueco de tensión. 7 de noviembre de 2009 .}

El 7 de noviembre de 2009 a las 11:55 una falta se registró en la subestación de Olmedilla, resultando un hueco de tensión y produciendo una pérdida instantánea de 825 MW de potencia eólica. Para compensar esta caída, se produjo un desvió de la conexión UCTE de 1006 MW para mantener el balance. Esta situación fue corregida con generación convencional a los 4 minutos. En la figura 4.18 se muestra la potencia eólica y el intercambio UCTE durante el hueco.

\subsubsection{Huecos de tensión. 19-20 de marzo de 2007 .}

Un ejemplo del efecto sobre la producción eólica que producen los huecos de tensión se puede apreciar en la figura 4.19 que muestra la evolución de la producción eólica el día 19 de marzo de 2007. Durante ese día se produjeron 4 recortes debidos a huecos de tensión de 550, 450, 1000 y $1000 \mathrm{MW}$ respectivamente.

En la figura 4.20 se muestra como afectó cada uno de los huecos a los nueve parques estudiados durante 2007 (WF1-WF9). Se aprecia que los huecos afectan a uno u otro parque según la zona en la se produjeron. Ademas se muestra que durante algunos huecos los parques se recuperan una vez superada la falta, mientras que otros se quedan desconectados, como es el caso del parque WF5 durante el hueco 3.

\subsection{Conclusiones}

En este capítulo se ha cuantificado, tanto la evolución como la situación actual de la variabilidad eólica en la red eléctrica española a través de los principales parámetros característicos de variabilidad y mediante ejemplos de eventos extremos que han acaecido en los últimos años, en los que el peso de la potencia eólica instalada tiene una importancia considerable. Este análisis se ha llevado a cabo usando los datos reales de producción y predicción, descritos en el capítulo 3, de la producción global agregada española. Se ha 
Capítulo 4. Características y efectos de la variabilidad de la potencia 114 eólica en España

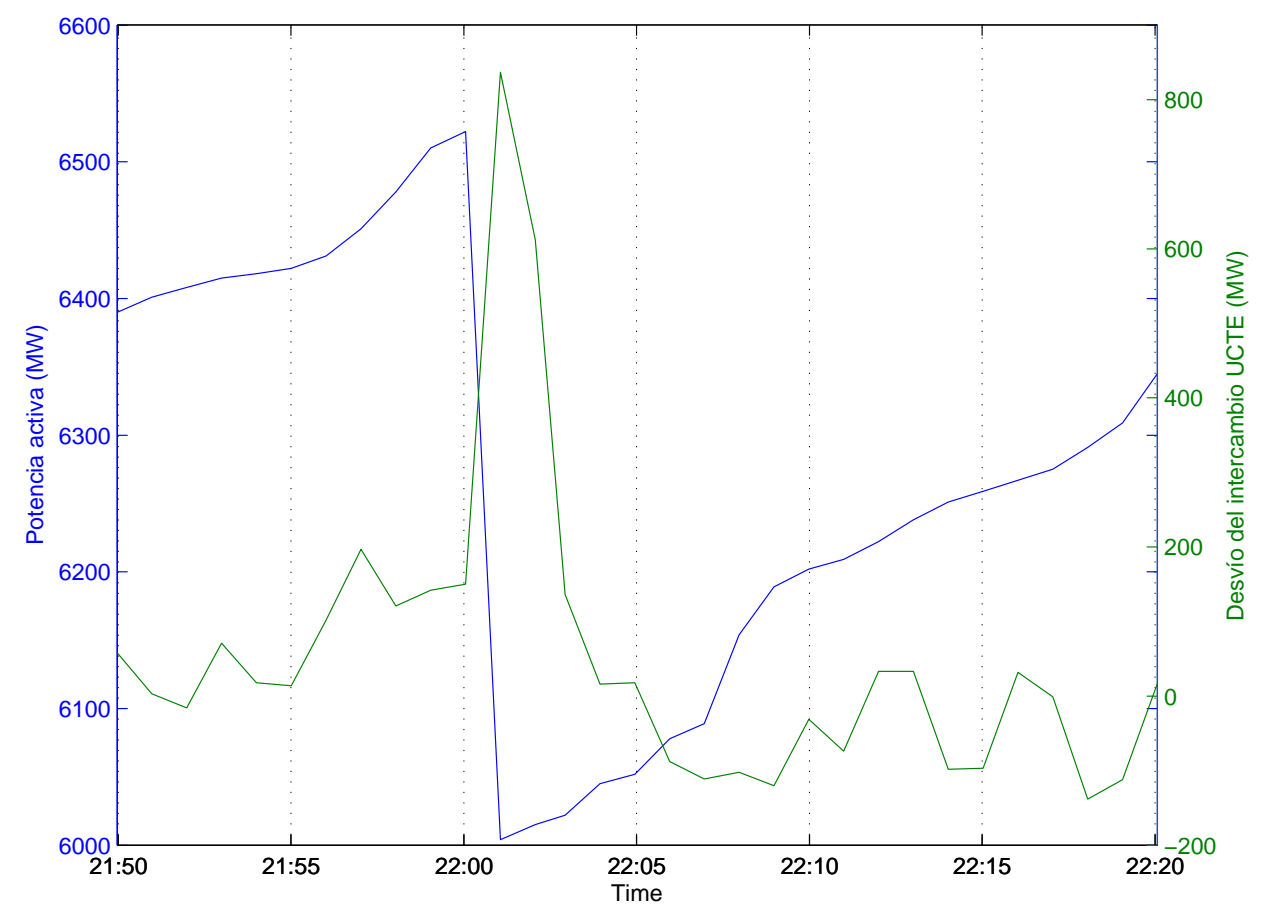

Figura 4.17: Generación eólica y desvio de la conexión UCTE durante el hueco del 30 de marzo de 2009.

estudiado la variabilidad según la época del año y el nivel de agregación existente. Así mismo se ha analizado el error de la predicción eólica, la cual es la herramienta principal en la gestión y operación de potencia eólica. Los errores obtenidos poseen valores relativamente altos especialmente cuando la producción es elevada, por lo que los desvíos, y por lo tanto, la reservas necesarias son consecuentemente altas. Finalmente, se indican eventos extremos característicos de diversos tipos que ofrecen una visión de los problemas puntuales más acuciantes a los que se debe hacer frente dentro de los efectos que plantea la variabilidad eólica en la red. 


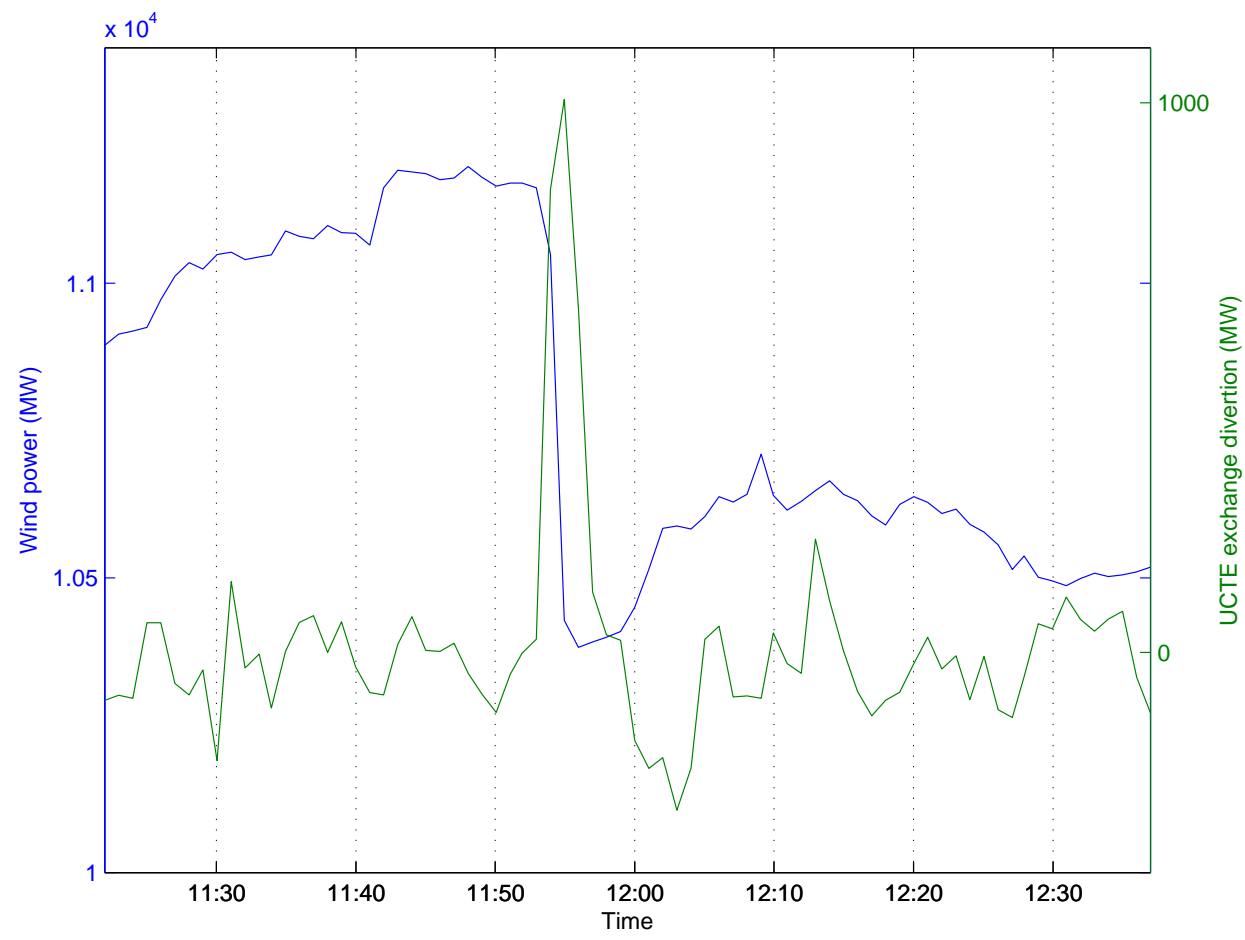

Figura 4.18: Generación eólica y desvío de la conexión UCTE durante el hueco del 7 de noviembre de 2009. 
Capítulo 4. Características y efectos de la variabilidad de la potencia eólica en España

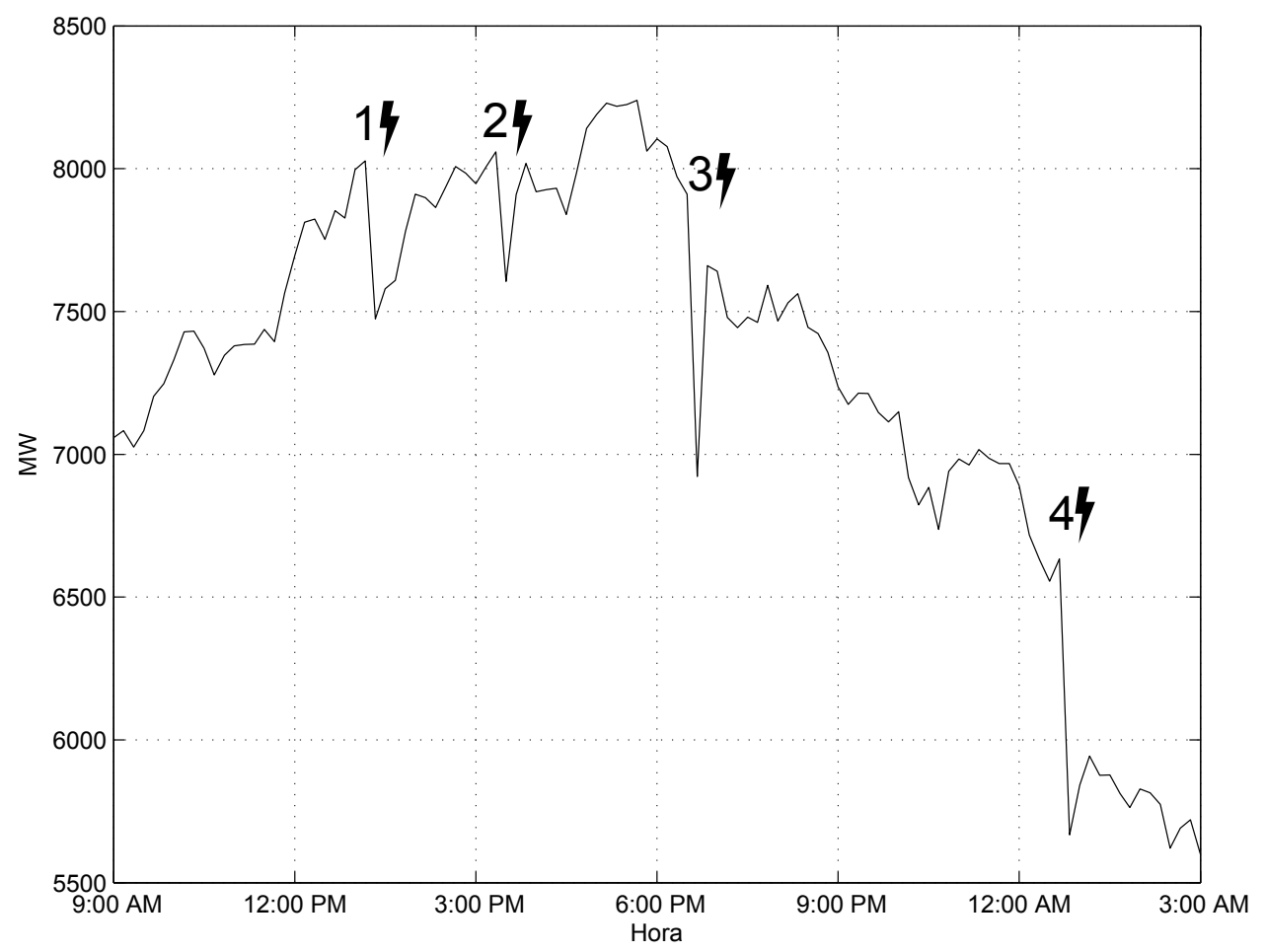

Figura 4.19: Generación eólica durante los huecos de los días 19 y 20 de marzo de 2007 


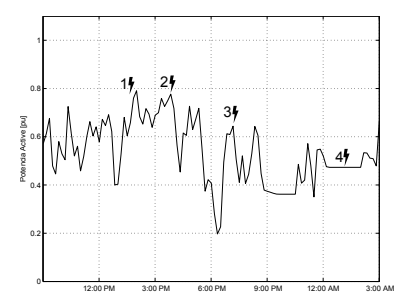

(a) WF1

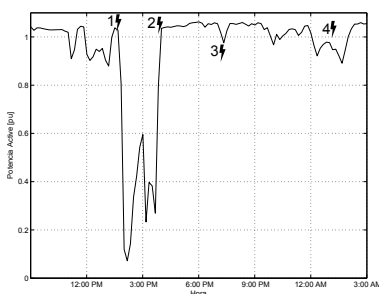

(d) WF4

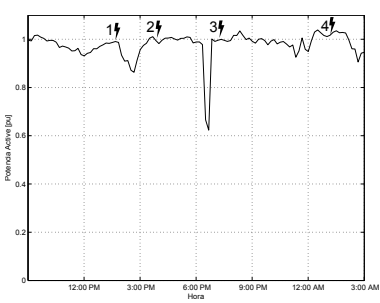

(g) WF7

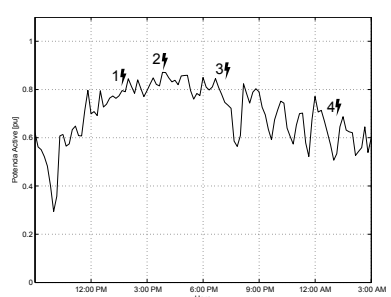

(b) WF2

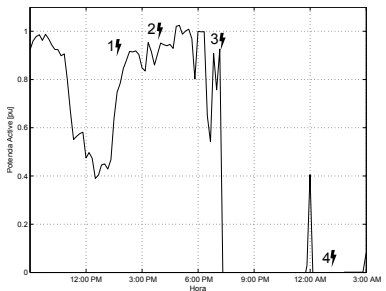

(e) WF5

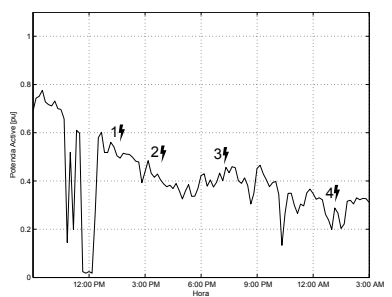

(h) WF8

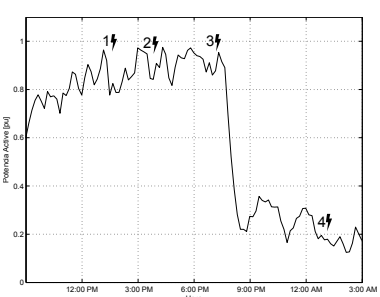

(c) WF3

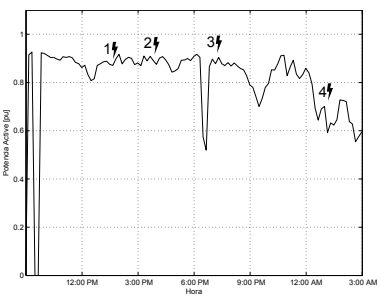

(f) WF6

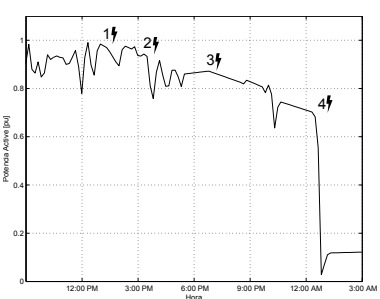

(i) WF9

Figura 4.20: Parques eólicos españoles y agregaciones consideradas. 



\title{
Capítulo 5
}

\section{Métodos de regulación de la potencia activa para la reducción de las fluctuaciones de potencia eólica}

\author{
El pesimista se queja del viento, el \\ optimista espera que cambie, el realista \\ ajusta las velas. \\ William George Ward
}

\begin{abstract}
RESUMEN: En este capítulo se presentan y desarrollan los principales métodos de control disponibles para la regulación de fluctuaciones de potencia eólica. Cada uno de los métodos es descrito y se analiza la reducción de las rampas en función de las pérdidas resultantes de su aplicación. Así mismo se proponen diferentes configuraciones del método PRL para optimizar la reducción de los valores de PNRR-99 y desviación estándar con las menores perdidas. También se muestran los resultados de la aplicación del método PRL a la producción global eólica española. A continuación se realiza un análisis similar a los anteriores pero utilizando el método con constante Delta. Como último método de reducción de fluctuaciones a estudiar se evalúa el uso del control de tormenta, para un parque eólico. Finalmente se indican las conclusiones derivadas de los resultados obtenidos.
\end{abstract}




\subsection{Introducción}

La regulación de potencia activa en parques eólicos está contemplada en el "grid code" danés Eltra (2004). En este documento se establecen diferentes funciones de regulación de la potencia activa de salida de un parque eólico. Los parques eólicos daneses deben ser capaces de limitar su producción a una cierta consigna entre el $20 \%$ y el $100 \%$ de su potencia nominal. Así mismo, la desviación entre el valor de consigna y la media en 5 minutos de la medida en el punto de conexión no puede superar el $\pm 5 \%$ de la potencia nominal del parque eólico. En cada parque eólico se coloca un controlador, llamado controlador de parque, el cual se encarga de asegurar que las órdenes de regulación se llevan a cabo en punto de conexión del parque y establece una conexión de forma que permita el control remoto de la producción de potencia activa. Adicionalmente, cada parque eólico debe cubrir las siguientes funciones de regulación de potencia activa desempeñadas por el controlador de parque:

- Constante absoluta de producción. Esta función consiste en la limitación de la potencia disponible ajustándola a una constante la cual es inferior a esta potencia disponible. La velocidad de regulación de esta función, tanto a subir como a bajar, debe oscilar y poder ajustarse en el intervalo entre el $10 \%$ y $100 \%$ de la potencia indicada por minuto. Su uso esta previsto durante periodos con una capacidad reducida de transporte en la red, por ejemplo debido a la puesta en servicio o sustitución de componentes en la red principal. Normalmente se opera reduciendo los niveles de potencia de los aerogeneradores disponibles.

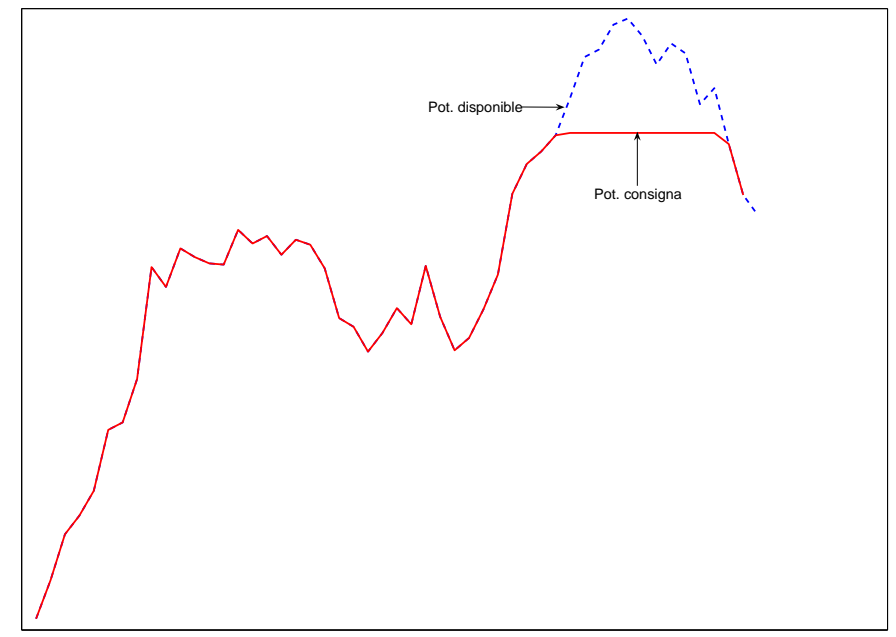

Figura 5.1: Regulación mediante constante absoluta

- Constante Delta de producción. Esta función ajusta la producción de 
forma que esta es limitada a un valor de la constante Delta por debajo de la producción disponible. El valor de Delta se establece en MW. Este tipo de regulación nos permite usar el parque para realizar regulación primaria si es necesaria, tanto en subfrecuencia como en sobrefrecuencia.

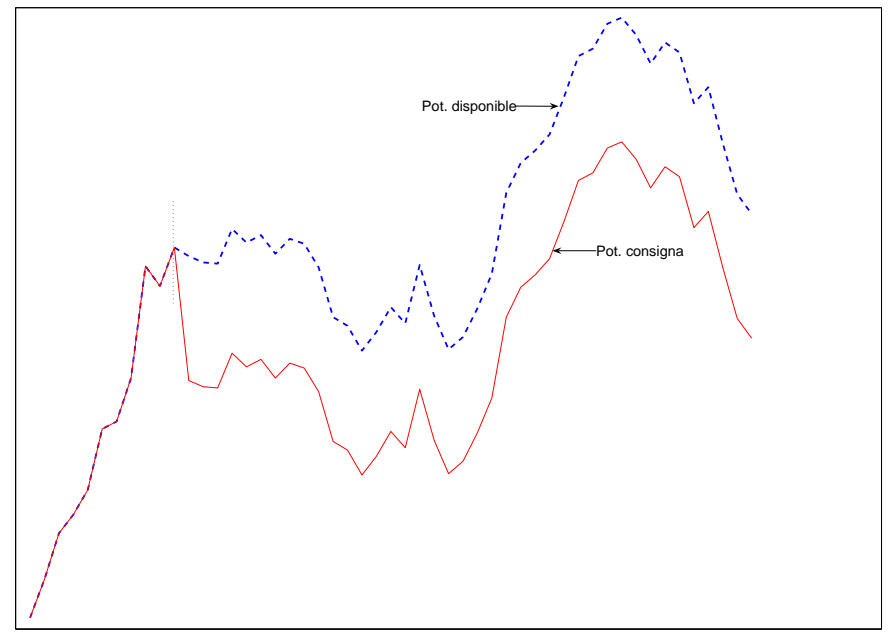

Figura 5.2: Regulación mediante constante Delta de producción

- Regulación de balance. Esta regulación implementa una regulación de potencia asegurando subidas o bajadas en la producción del parque cuando sea necesario. El cambio en la potencia se puede establecer opcionalmente con órdenes, parcialmente con el cambio deseado (cambio de $\pm M W)$ y parte con un gradiente de potencia deseado $(M W / \mathrm{min})$. La limitación finaliza cuando la potencia disponible es menor que la potencia de consigna. También es posible realizar una cancelación automática una vez ha discurrido el tiempo establecido y la producción regresa a la potencia disponible mediante un gradiente ajustable. Cuando se realiza la regulación de balance se permite que la producción exceda el valor de la potencia de consigna establecida con la constante absoluta de producción. Este tipo de regulación permite que el parque eólico participe en la regulación secundaria de una zona de la red,Kristoffersen (2005).

- Regulación de parada. La activación de esta función asegura que el parque eólico mantenga la potencia generada en ese instante de forma constante mientras sea posible, ya que cuando la velocidad del viento caiga será imposible llevarse a cabo. Cuando se cancela esta función, la producción vuelve al valor establecido con determinado gradiente que puede ser ajustado. 


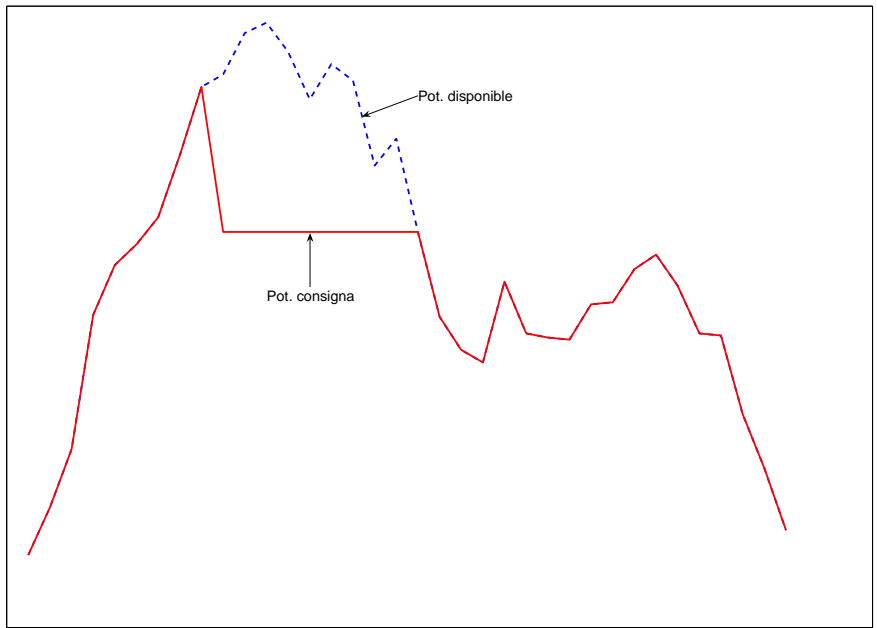

Figura 5.3: Regulación de balance sin cancelación automática

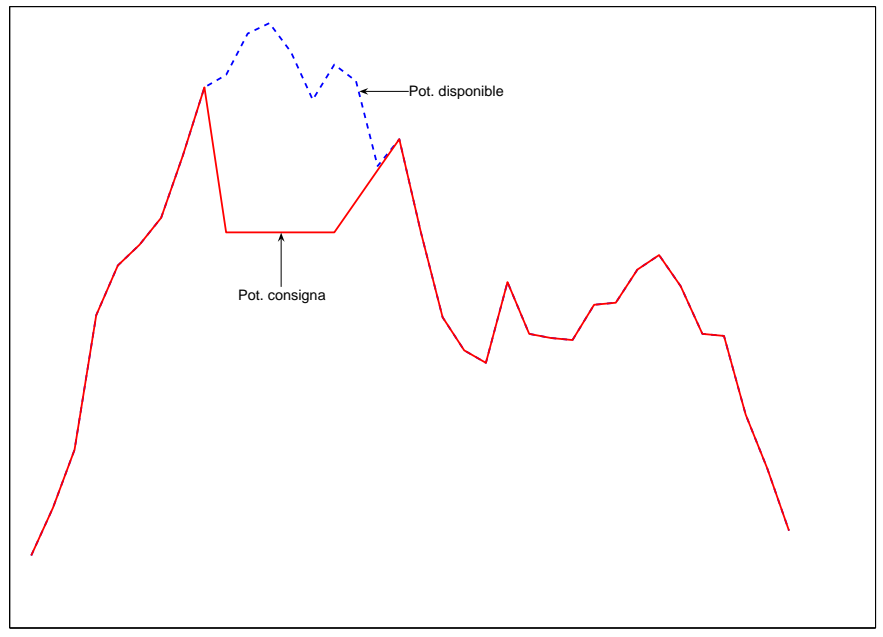

Figura 5.4: Regulación de balance con cancelación automática

- Constante de gradiente de potencia. Esta regulación previene que la producción del parque se incremente demasiado rápido cuando la velocidad de viento aumenta a causa de ráfagas o cuando el parque arranca con una velocidad de viento alta. Si la velocidad del viento disminuye, esta función no tiene efecto, a no ser que este también en curso la función de constante Delta. El operador del sistema es el encargado de establecer los valores máximos permisibles para los gradientes.

- Protección del sistema. El controlador de parque, mediante una señal externa, debe ser capaz de realizar un rápido descenso de la potencia del parque de forma que el sistema quede protegido. El descenso se 


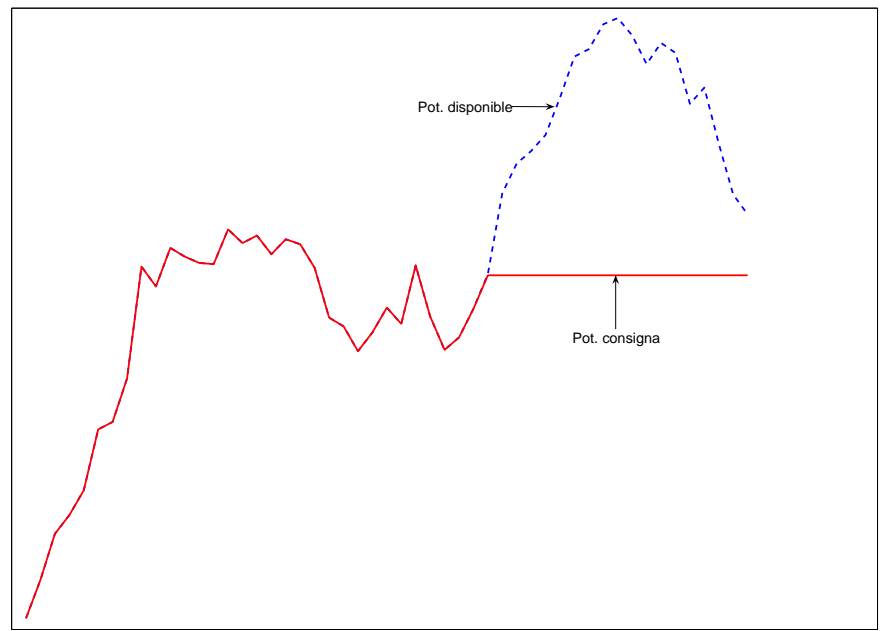

Figura 5.5: Regulación de parada

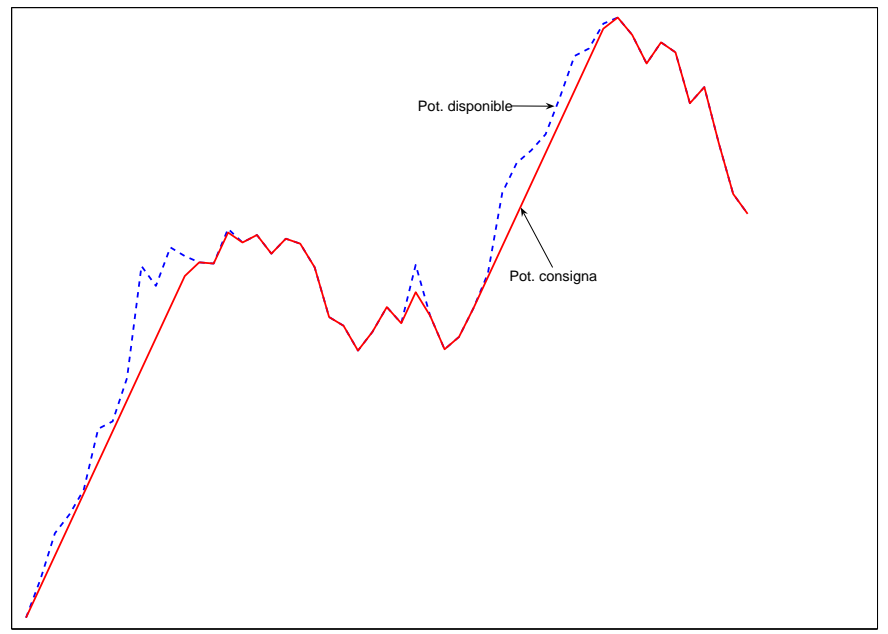

Figura 5.6: Regulación con constante de gradiente de potencia

debe llevar a cabo con una velocidad preestablecida, también se puede determinar con antelación la máxima cantidad de potencia regulable. Esta regulación se mantendrá hasta que la máxima cantidad regulable se alcance mientras que está activa la señal externa que activo esta función. Una vez que la señal externa finalice la potencia vuelve a la configuración original. Esta protección puede ser reseteada de forma manual. La regulación descendente puede variar la potencia desde la potencia nominal hasta la parada en un máximo de 30 segundos.

- Regulación con control de frecuencia. El control individual de un aerogenerador realiza una regulación automática de la frecuencia con un 


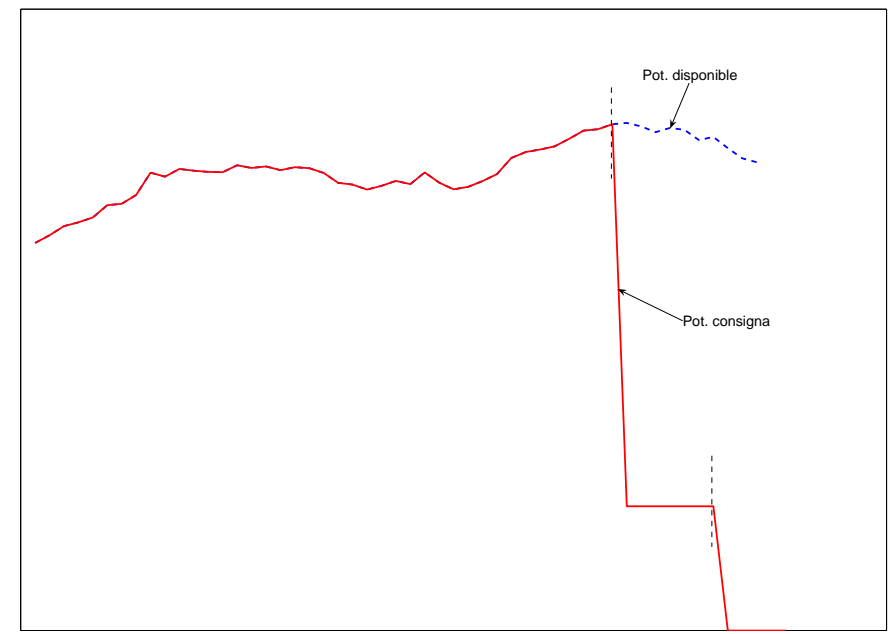

Figura 5.7: Regulación para protección del sistema

cambio en la producción dependiendo de la frecuencia de la red. A partir del controlador del parque es posible establecer los parámetros de todo el parque. existen dos casos en la regulación de la frecuencia, en el primero no existe un recorte en la producción por lo que solo se puede realizar una regulación descendente de la producción, en el segundo caso también se puede realizar una regulación ascendente, además de la descendente, debido a que la producción ha sido previamente recortada. Las características del control de frecuencia se pueden establecer de forma colectiva para todo el parque.

En las funciones descritas anteriormente existe una prioridad entre ellas. El orden de preferencia es el siguiente:

1. Protección del sistema.

2. Regulación con control de frecuencia.

3. Regulación de parada.

4. Regulación de balance.

5. Constante de gradiente de potencia.

6. Constante absoluta de producción.

7. Constante Delta de producción.

Otro país en el que también se ha tenido en cuenta la necesidad de controlar la potencia activa de salida de los parques eólicos debido a la gran 
penetración de energía eólica en su sistema eléctrico es Irlanda. El "grid code" irlandés Eirgrid (2009) establece que el sistema de control del parque eólico debe ser capaz de controlar los valores de rampa de la potencia activa de salida con un valor de rampa de un máximo de MW por minuto establecido por el operador del sistema. Así mismo, define dos configuraciones de rampa máxima, una de rampa media para un minuto y otra para una rampa media de 10 minutos. Estas limitaciones de rampa deben ser aplicables para cualquier rango de operación incluido el arranque, el régimen nominal y la desconexión. El operador del sistema permite que se excedan estos valores de rampa en caso de caída de la velocidad del viento o si es necesaria una respuesta para regular la frecuencia. Además, debe ser posible la variación de estos parámetros de forma independiente y en un rango entre 1 y $30 \mathrm{MW}$.

En el caso de España, para la generación no gestionable, en la que se encuentra la energía eólica, solo es obligatoria la regulación de potencia activa en caso de que tenga relevancia para la seguridad del sistema REE (2009). El Operador del Sistema queda fuera de asegurar, supervisar o valorar los mecanismos de control en estas instalaciones en cometidos que difieran a la seguridad del sistema. El mecanismo más utilizado de regulación es la supresión de "Excedentes de generación no integrables en el Sistema" como se vió en el apartado 4.7.2 y únicamente en el caso de asegurar la seguridad del sistema. Esto impide que los mecanismos de control descritos anteriormente y utilizados en otros sistemas nacionales sean aplicados. Del mismo modo no se utiliza la generación eólica para la optimización de la generación global del sistema ni esta contribuye en la regulación primaria y secundaria del sistema. En el sistema eléctrico español el sistema GEMAS es el encargado de calcular la máxima cantidad de potencia eólica que puede ser conectada a la red con una cadencia de 10 minutos de forma que se cumplan las condiciones de seguridad según la cantidad de reservas disponibles. Por tanto, este sistema propone la cantidad de potencia eólica conectable a partir de la reservas disponibles, no establece las reservas según la producción eólica.

De los tipos de regulación disponibles descritos anteriormente, hay dos que pueden resultar útiles para la reducción de los efectos que producen las fluctuaciones, concretamente la limitación del gradiente tanto positivo y como negativo, y la constante Delta de producción. Esto se pone de manifiesto en Vigueras-Rodríguez (2008). Así, estas herramientas se pueden utilizar para reducir la variabilidad de la energía eólica y por lo tanto las reservas adicionales del sistema. El uso de estos métodos de control sobre la potencia eólica puede disminuir las fluctuaciones más extremas en magnitud y frecuencia en escalas de tiempo tanto pequeñas como para eventos prolongados.

Cuando una de estas estrategias es ejecutada, la potencia resultante de salida $\left(P_{\text {curt }}\right)$, que ha sido recortada, es menor o igual que la potencia original disponible $\left(P_{\text {aval }}\right)$. La diferencia entre estos dos valores es la potencia de 
pérdidas $\left(P_{\text {leak }}\right)$ causadas por las estrategias de regulación. La relación entre estos valores se indica en la ecuación 5.1.

$$
P_{\text {leak }}(t)=P_{\text {aval }}(t)-P_{\text {curt }}(t)
$$

Para el análisis de estas estrategias se deben tener en cuenta las pérdidas totales asociadas a su uso calculadas según la ecuación 5.2.

$$
E_{\text {leak }}=\int_{T_{s}} P_{l e a k} d t
$$

Estas regulaciones conllevan unas perdidas en la producción por lo que el uso frecuente de esta opción debe ser sopesado con otras medidas, en otras unidades de producción, en términos de coste y efectividad.

Otro método de control aplicable para la reducción de la variabilidad de la potencia eólica generada es el control de tormenta, denominado en la literatura como "Storm Control". Este tipo de control consiste en variar el control del ángulo de incidencia del viento de forma que para velocidades mayores que la velocidad de corte se obtenga una potencia de salida que se irá reduciendo progresivamente al aumentar la velocidad de viento, en lugar de producirse la desconexión por protección por sobrevelocidad.

En Cutululis et al. (2010) se propone el uso de los tipos de controles de tormenta mostrados en la figura 5.8. Se simulan las curvas de potencia con control de potencia para diferentes escenarios de viento y con periodos de 15 y 30 minutos en varios parque eólicos marinos daneses. Los resultados indican una reducción considerable de las rampas mas negativas, especialmente usando la curva de potencia con control de tormenta suave, mostrada en la figura 5.8a.

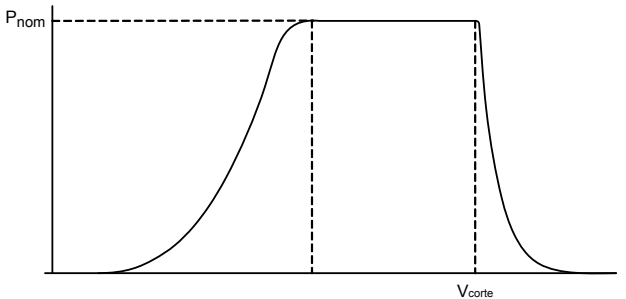

(a) Control de tormenta suave

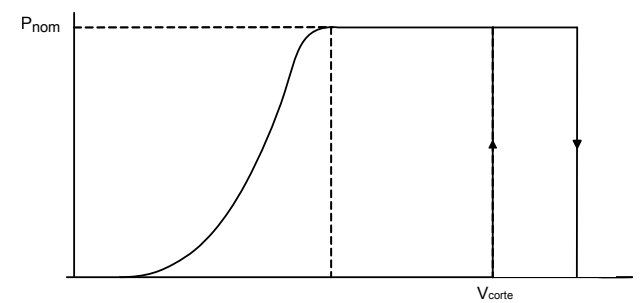

(b) Control de tormenta con histéresis

Figura 5.8: Tipos de control de tormenta. Cutululis et al. (2010).

Por otra parte, en Markou y Larsen (2009) se estudian diferentes tipos de control velocidad de viento/potencia que incluyen control de tormenta en contraposición con el sistema tradicional. En esos tipos de control, la velocidad de giro del rotor y la potencia se va reduciendo progresivamente por encima de la velocidad de corte, en este caso $25 \mathrm{~m} / \mathrm{s}$. También se aprecia que al usar estos métodos es posible limitar las cargas mecánicas en el rotor pero 
la cargas debidas a la vibración de la torre se incrementan. Como resultado, se analizan dos algoritmos de control para la reducción adicional de cargas. En primer lugar se usa un algoritmo para amortiguar las cargas frontales de la torre basado en la regulación colectiva del ángulo de incidencia a partir de las medidas de la aceleración frontal en la parte superior de la torre. Como segundo caso, se considera un algoritmo de amortiguación de las cargas laterales de la torre. Este consiste en la demanda adicional de par en función de las mediciones de la aceleración lateral en la parte superior de la torre. El uso de estos algoritmos de control conlleva una importante reducción de las cargas indicadas cuando se usa el control de tormenta, alcanzando valores inferiores a los obtenidos con curvas de potencia tradicionales. Todo esto indica la posibilidad de utilizar el control de tormenta sin que existan cargas adicionales derivadas de su uso que reduzcan la vida útil del aerogenerador o que puedan plantear un peligro adicional en su funcionamiento.

En la actualidad ya existen aerogeneradores que implementan, de base, sistemas de control de tormenta. Un ejemplo es el modelo Enercon E-70 que incluye un sistema de control de tormenta suave, como el que se aprecia en la figura $5.8 \mathrm{a}$. Su velocidad de corte está entre 28 y $34 \mathrm{~m} / \mathrm{s}$ y se ha implementado un control para que a partir de una cierta velocidad denominada $V_{\text {storm }}$ la velocidad rotacional se reduce y de esta forma la potencia de salida disminuye progresivamente. Esto se consigue variando el ángulo de incidencia del viento sobre las palas tal y como se describe en Enercon (2011). De esta forma, si la velocidad de viento disminuye, el ángulo de incidencia se reajusta de forma que el aerogenerador vuelve a operar en condiciones normales a potencia nominal.

En los siguientes apartados se van a analizar los dos tipos de regulación para conocer su capacidad de reducción de la variabilidad de la producción eólica utilizando los datos descritos en el capitulo 3, tal y como se ha estudiado en Martín-Martínez et al. (2013). A continuación, se analizará el uso de control de tormenta sobre un parque eólico. Finalmente se exponen las oportunas conclusiones del capítulo.

\subsection{Métodos de Limitación de Rampa Positiva (PRL)}

La Limitación de Rampa Positiva (PRL) consiste en limitar el gradiente máximo de incremento de la producción a un valor establecido como se indica en la ecuación 5.3. Cuando se aplica este método de control a una serie temporal de producción eólica, la serie resultante posee unos valores iguales o inferiores a la producción disponible, siendo la perdidas de potencia resultantes las establecidas en la ecuación 5.1 y si se integran esas pérdidas en el tiempo se obtienen las pérdidas de energía totales, tal y como se refleja en la ecuación 5.2. El método PRL aporta, de forma adicional, varias ventajas en la operación de un parque eólico como son la prevención de un gran 
incremento de la producción del parque debido a un aumento muy rápido de la velocidad del viento, y la limitación cuando el parque arranca bajo unas condiciones de una alta velocidad de viento. Si la velocidad de viento disminuye y por lo tanto la potencia de salida decrece, entonces la limitación PRL no tiene ninguna función. Este método puede aplicarse tanto a parques eólicos individuales como a agregaciones de los mismos para la reducción de las rampas presentes en sus series temporales.

La aplicación de este método de control se describe en el diagrama de la figura 5.9 y se resume en la ecuación 5.3. La potencia disponible en cada instante y la rampas máxima fijada son los parámetros de entrada, mientras que la potencia recortada resultante para cada instante es el único parámetro de salida.

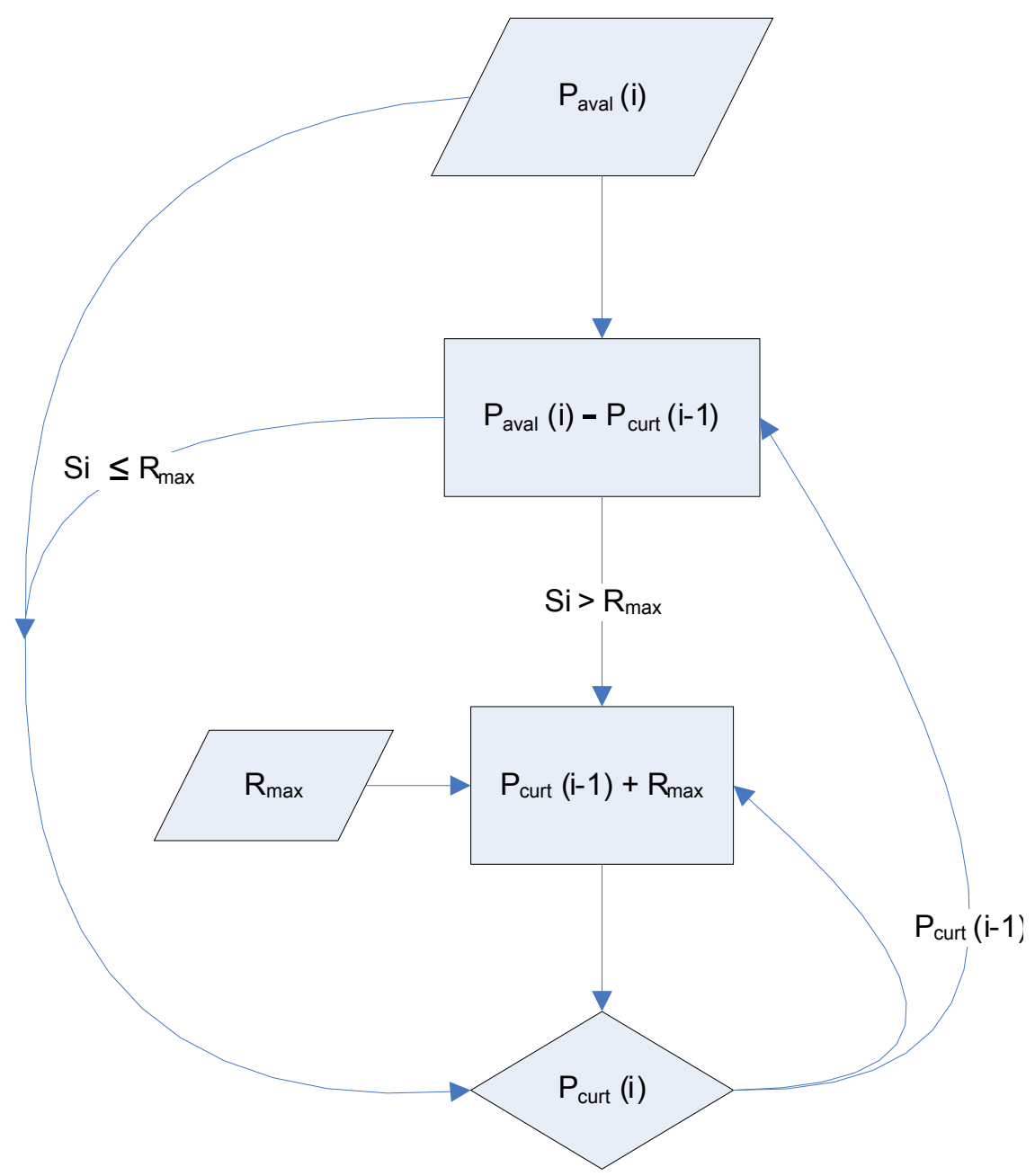

Figura 5.9: Esquema de aplicación de la regulación PRL a las series temporales 


$$
P_{\text {curt }}(i)= \begin{cases}P_{\text {aval }}(i) & \text { if } P_{\text {aval }}(i)-P_{\text {curt }}(i-1) \leq R_{\text {max }} \\ P_{\text {curt }}(i-1)+R_{\text {max }} & \text { if } P_{\text {aval }}(i)-P_{\text {curt }}(i-1)>R_{\text {max }}\end{cases}
$$

Siendo $P_{\text {curt }}$ la potencia resultante al aplicar el método PRL a un parque o una agregación de parques, $P_{\text {aval }}$ representa la potencia máxima disponible en un parque o una agregación de parques y $R_{\max }$ es el límite máximo de gradiente positivo.

Un ejemplo de la relación entre las pérdidas resultantes de aplicar esta regulación en función de la rampa máxima $R_{\max }$ aplicada se muestran en la figura 5.10.Se aprecia que existe una primera zona de 0 a $0.01 \mathrm{pu} / \mathrm{min}$ en la que las pérdidas decrecen de forma drástica al aumentar el valor de $R_{\max }$ y una segunda zona para valores superiores a $0.01 \mathrm{pu} / \mathrm{min}$ en la que las pérdidas apenas disminuyen al aumenta el valor de $R_{\max }$.

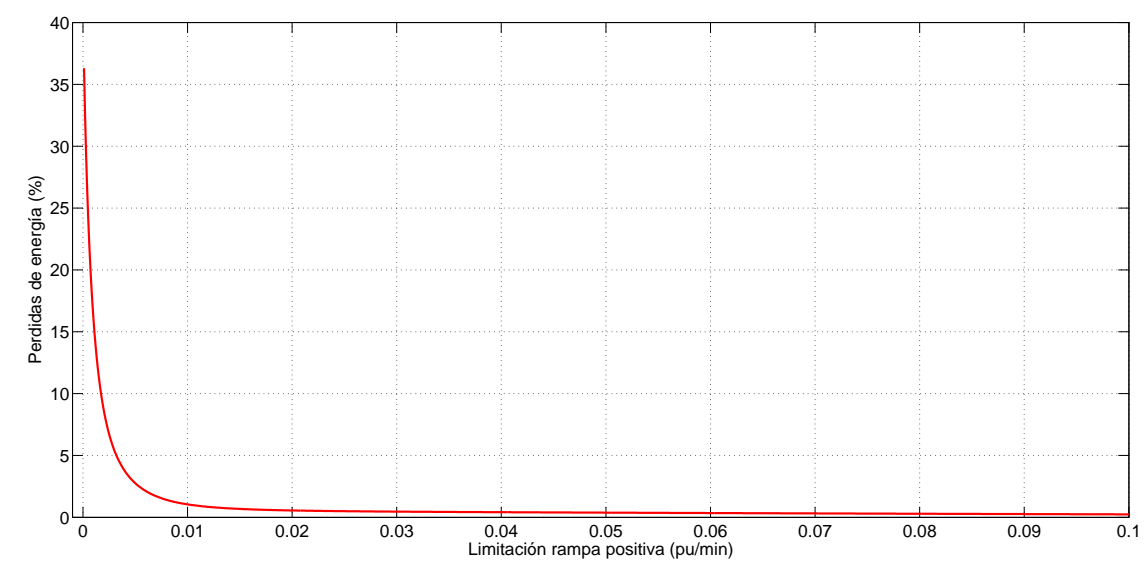

Figura 5.10: Ejemplo de pérdidas resultantes de la aplicación de la regulación PRL

Por su definición este método describe mejores comportamientos en eventos en los que se presenta un aumento rápido de potencia seguido, en un espacio de tiempo relativamente corto, de una caída hasta un nivel similar al valor previo al aumento. Este tipo de evento fue definido en la sección 2.3.4 como ráfaga. Así, en una ráfaga se consiguen importantes reducciones de las rampas tanto positivas como negativas, usando el método PRL. También se consigue una considerable reducción de las rampas positivas, fijándose el máximo como $R_{\max }$ en eventos de aumento persistente de la potencia eólica.

En Vigueras-Rodríguez et al. (2009), el método PRL es aplicado a un parque eólico marino, mostrando su utilidad en la reducción y suavizado de las fluctuaciones presentes ante la variabilidad de la velocidad de viento, especialmente en eventos como ráfagas. No obstante, no se había evaluado la aplicación de este método sobre un conjunto de parques eólicos. 
En los siguientes apartados se desarrolla la aplicación de este método de regulación en diferentes configuraciones para determinar el nivel de reducción de las fluctuaciones, con especial atención a las rampas negativas más extremas. La primera configuración de PRL usada, denominada PRL individual, consiste en la aplicación de la ecuación 5.3 sobre la serie de potencia de cada parque eólico de forma individual para finalmente sumar la producción recortada resultante. En contraposición, se propone otra configuración, la cual consiste en la aplicación de la ecuación 5.3 sobre la serie de potencia de toda la agregación de parques, la cual se obtiene al sumar la potencia de todos los parques pertenecientes a la agregación. A esta configuración se le denomina PRL global. Estas y otras estrategias se estudian en las secciones siguientes con el objetivo de obtener la mayor reducción de las rampas negativas más extremas con las mínimas pérdidas derivadas.

\subsubsection{PRL aplicado a parques de forma individual}

En este apartado se ha llevado a cabo la regulación PRL de los parques estudiados y descritos en el capítulo 3 de forma individual aplicando a cada uno de ellos la misma limitación de rampa y comparando la reducción de las rampas negativas de la producción global recortada con respecto a la producción global disponible. Se han considerado dos agregaciones, por un lado la agregación de los parques del grupo 1 de datos descritos en la tabla 3.1 y dispuestos tal y como indica la figura 3.1a y por otro lado la agregación de los parques del grupo 2 descritos en la tabla 3.2 y con una distribución tal y como indica la figura $3.1 \mathrm{~b}$.

La reducción ha sido sopesada en función de la energía perdida al realizar la regulación y se manejan unos límites de pérdidas que abarcan del $1 \%$ al $5 \%$, considerándolos asumibles dentro de la búsqueda de la obtención de la máxima producción.

El método PRL individual se aplica tal y como se describe en la figura 5.11. En primer lugar se aplica el método PRL a cada parque de la agregación tal y como se indica en el esquema 5.9. A continuación se suman las potencias de salida de cada parque para obtener la potencia recortada de toda la agregación.

En la figura 5.12, se muestra, para un cierto periodo de tiempo, el recorte sobre la producción total disponible de los parques estudiados de la regulación PRL aplicada a los parques de forma individual. Se aprecia que su comportamiento se asemeja en ciertos tramos a una regulación mediante constante Delta de la producción global, estableciéndose un margen de potencia, más o menos constante, a subir con respecto a la potencia disponible. También se observa una acentuación de los valles de producción lo que restringe la mejora de la reducción de las rampas negativas de la producción eólica. Esto se debe a la limitación de la rampa en ciertos parques que aumentan su producción cuando la producción total de la agregación está disminu- 


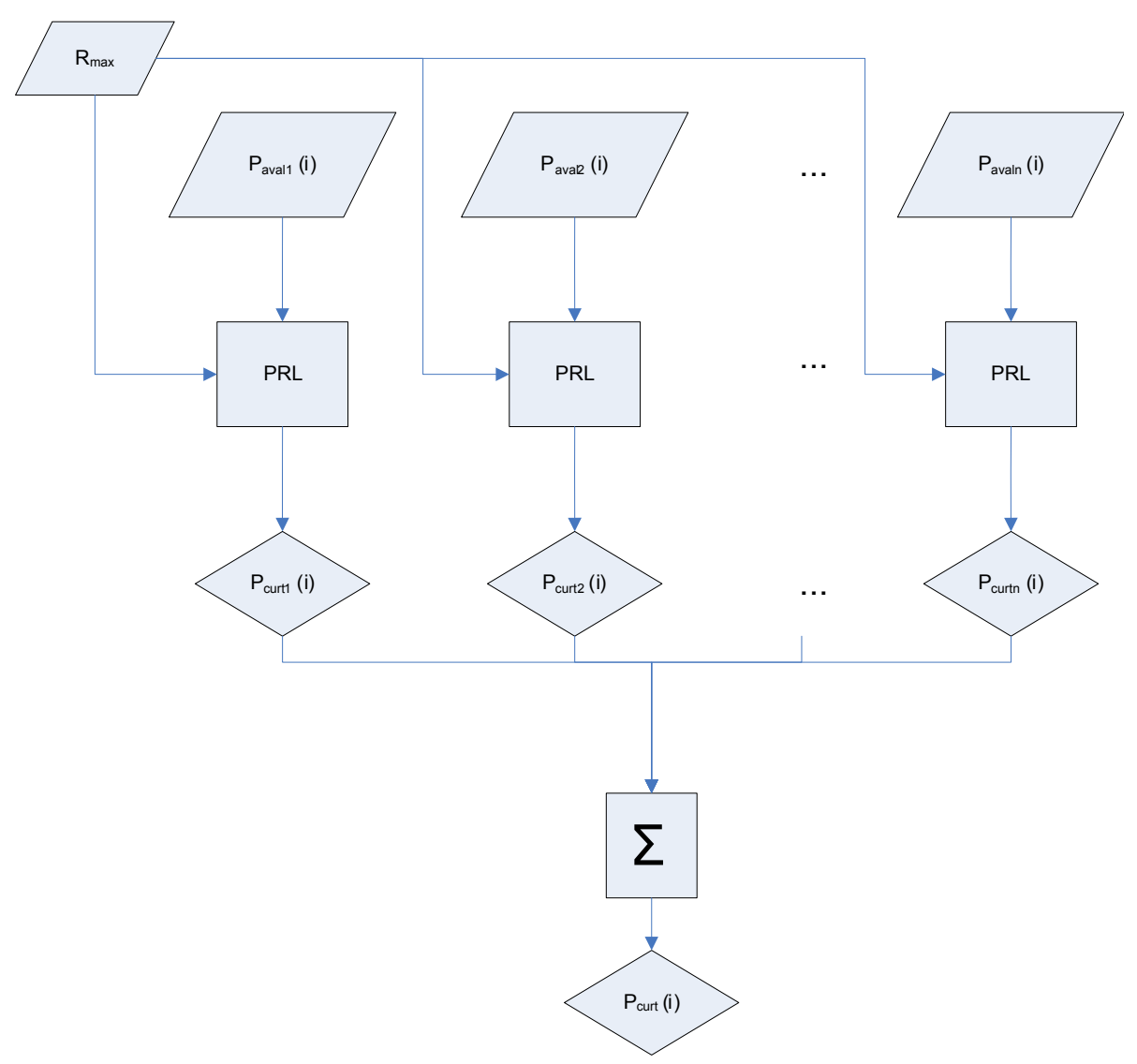

Figura 5.11: Esquema de aplicación de la regulación PRL individual

yendo. Existe un margen de mejora de este tipo de regulación mediante la no aplicación de PRL cuando la producción global realiza rampas negativas, especialmente en las zonas con valles de producción.

Los resultados de la aplicación de esta configuración de PRL se muestran en las figuras 5.13 y 5.14. En estas figuras se observa la reducción de los valores de PNRR-99 y de desviación estándar en función de las pérdidas resultantes. Se puede apreciar como la reducción de ambos parámetros aumenta considerablemente según aumentan las pérdidas pero al alcanzar un cierto nivel ese aumento se satura y el margen de reducción que se obtiene al incrementar las pérdidas es cada vez menor.

\subsubsection{PRL aplicado a la producción agregada global}

En este apartado la regulación PRL se aplica a la producción global de los parques estudiados de forma directa, estableciendo una rampa máxima para la generación conjunta de los parques. El objetivo de esta regulación es también el de reducir las rampas negativas presentes. El método se aplica 


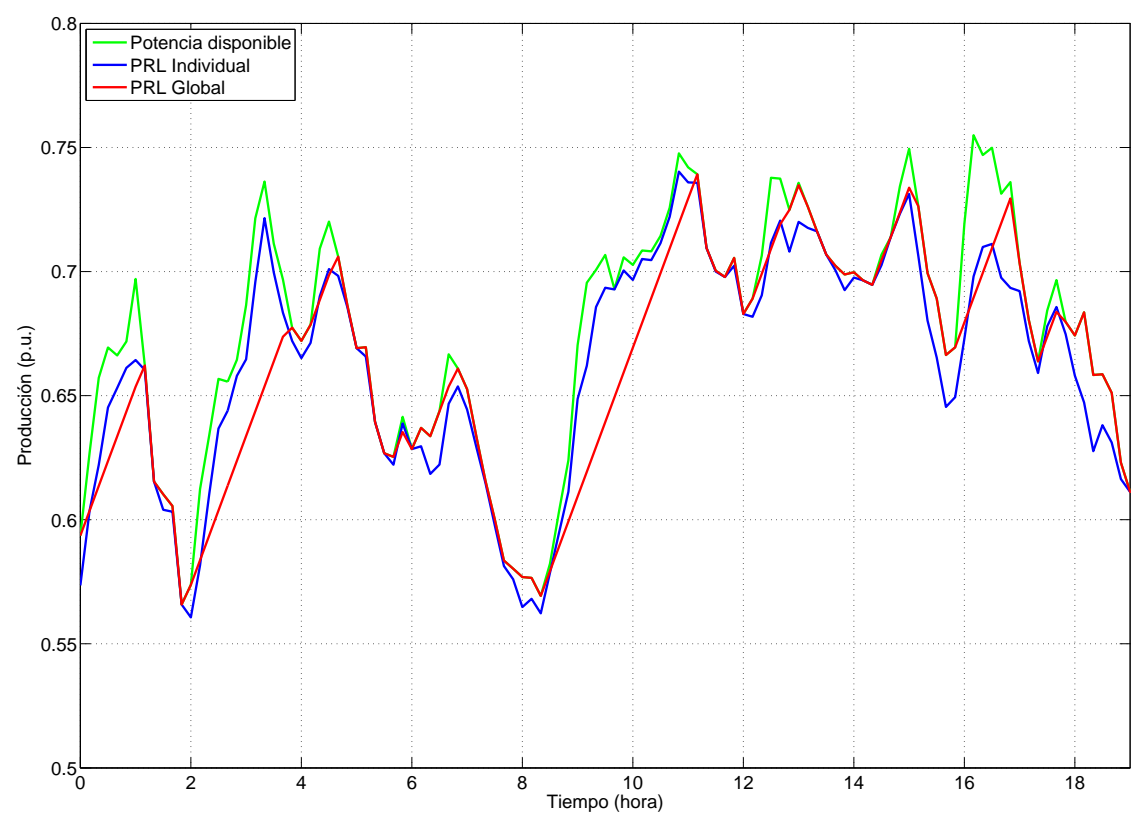

Figura 5.12: Comparativa de tipos de regulación y producción disponible(I)

directamente sobre la serie temporal de producción global de la agregación correspondiente usada también en el apartado anterior, tal y como indica el esquema 5.9. Del mismo modo que en los ejemplos anteriores, la reducción se sopesa con la energía perdida al realizar la regulación y se manejan unos límites de pérdidas que abarcan del $1 \%$ al $5 \%$.

En la figura 5.12 se comparan los métodos PRL global y el PRL individual. En la comparación se aprecia que usando el método global no existe diferencia entre la serie temporal resultante y la serie temporal disponible cuando la rampa original es negativa. También se muestra que con esta configuración no se produce el efecto de acentuación de los valles que se producía en el PRL individual. Por el contrario, no resulta eficiente reduciendo las rampas negativas cuando las rampas negativas pronunciadas no se producen seguidamente a las rampas positivas, tal y como ocurre entre las horas $16 \mathrm{y}$ 17.

Las figuras 5.13 y 5.14 muestran el porcentaje de reducción de los parámetros PNRR-99 y desviación estándar derivado de la regulación PRL global en comparación con la producción disponible y la reducción de las rampas negativas dependiendo de las pérdidas de energía. El nivel de reducción del PNRR-99 usando PRL global es ligeramente inferior que usando PRL individual para perdidas mayores al $1 \%$. Por el contrario, el porcentaje de reducción de la desviación estándar es muy superior utilizando el PRL global para todo el rango de pérdidas. Esto se debe a que el PRL individual únicamente funciona mejor en las reducción de las rampas negativas más 


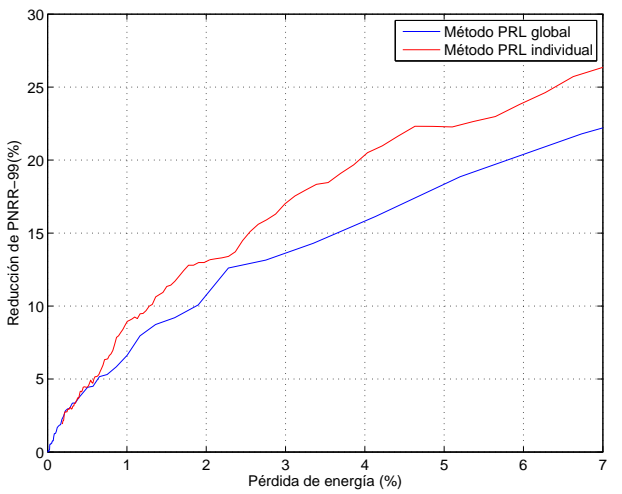

(a) Datos 2007

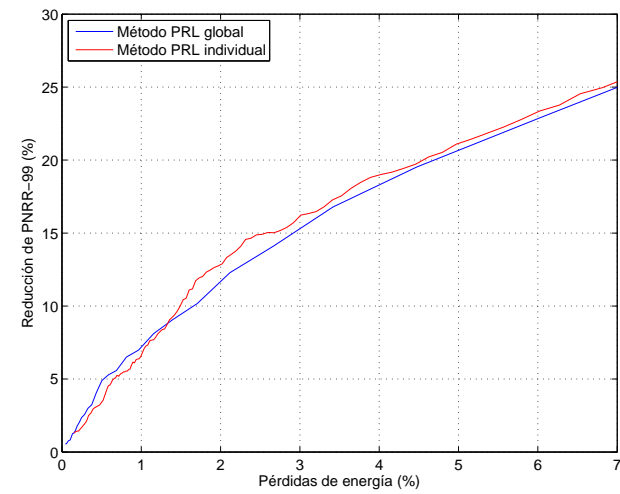

(b) Datos 2008

Figura 5.13: Reducción de $99 \%$ de rampa negativa y pérdidas para regulación PRL individual y global

extremas. En la figura 5.15 se indican los porcentajes de reducción de para todos el rango de valores PNRR con unas pérdidas del 1\%. De esta manera se puede apreciar que, excepto en los valores más extremos, los porcentajes de reducción son superiores utilizando el método PRL global.

\subsubsection{PRL aplicado según rangos de potencia}

Las fluctuaciones eólicas varían dependiendo del rango de potencia de funcionamiento del aerogenerador o parque, tal y como se ha establecido en el apartado 2.3.2. Las mayores fluctuaciones se presentan a carga parcial y normalmente en rangos de 0.4 a 0.8 p.u.. En este apartado se propone la aplicación de la regulación de limitación de rampa positiva (PRL) con una rampa máxima más limitada cuando la producción sea parcial y se encuentre en el rango entre 0.4 y 0.8 p.u.. De este modo se establecen dos valores de $R_{\max }$ en función del rango de potencia en el que se encuentre el parque o la agregación.

En caso de una producción parcial entre 0.4 y 0.8 p.u. se aplica una rampa máxima positiva más severa, mientras que si la producción se sitúa entre 0 y 0.4 p.u. o mayor de 0.8 p.u., la rampa máxima positiva puede ser más pronunciada.

En la figura 5.16 se aprecia este tipo de regulación aplicada de forma individual sobre cada uno de los parques y de forma global aplicada sobre la producción agregada de todos los parques, estableciendo una comparación con el método de $R_{\max }$ fija. Se observa el cambio de rampa máxima cuando se alcanza el 0.4 p.u. de la producción global en el caso del PRL aplicado a la producción agregada. En el caso de la aplicación individual el comportamiento es similar a la regulación con una rampa positiva máxima 


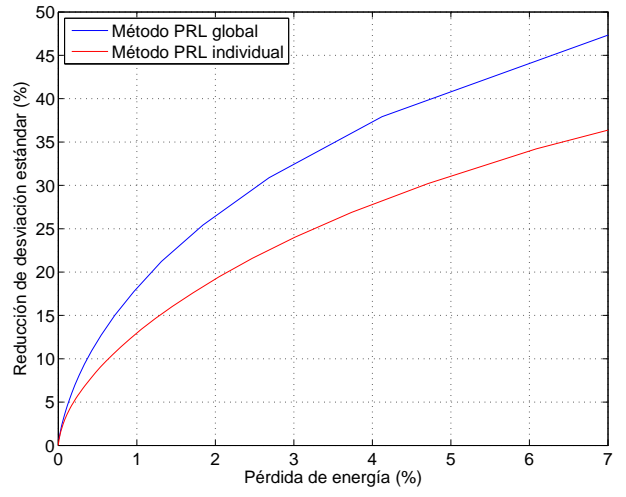

(a) Datos 2007

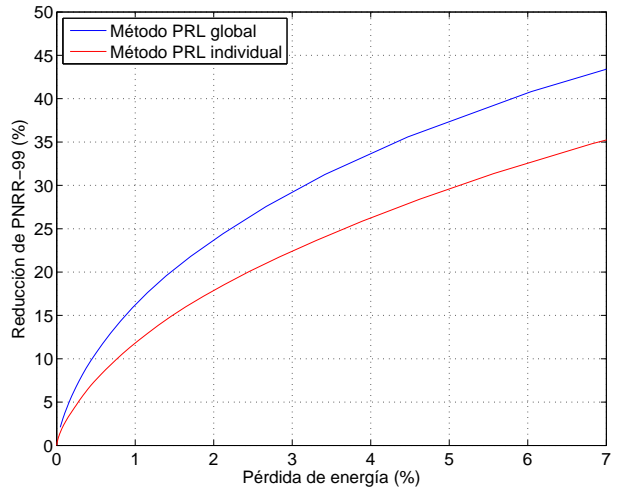

(b) Datos 2008

Figura 5.14: Reducción de la desviación estándar y pérdidas para regulación PRL individual y global

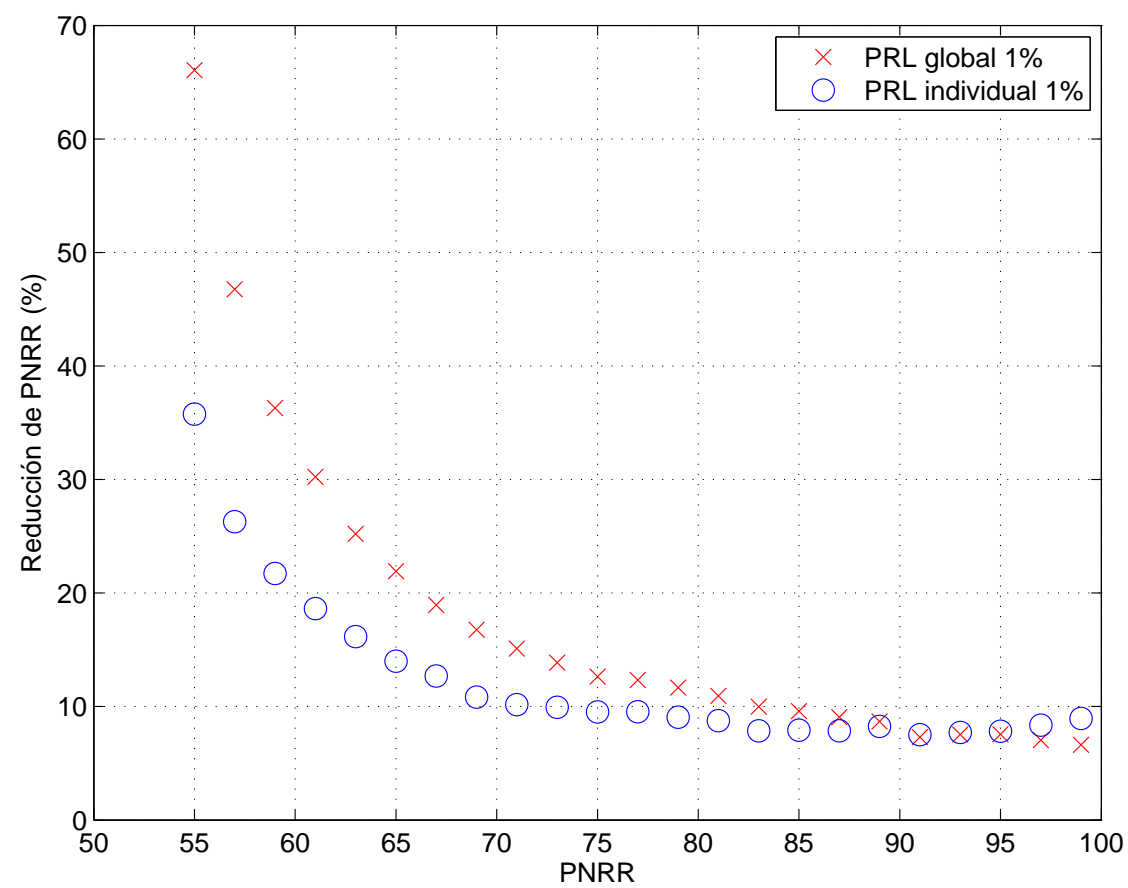

Figura 5.15: Reducción de rampas negativas PNRR usando PRL individual y global con unas pérdidas de energía del $1 \%$

fija.

La comparativa de los resultados obtenidos entre las diferentes opciones de regulación propuestas hasta el momento se establece en la figura 5.17. La regulación con rampa máxima variable permite una ligera mejora sobre los 


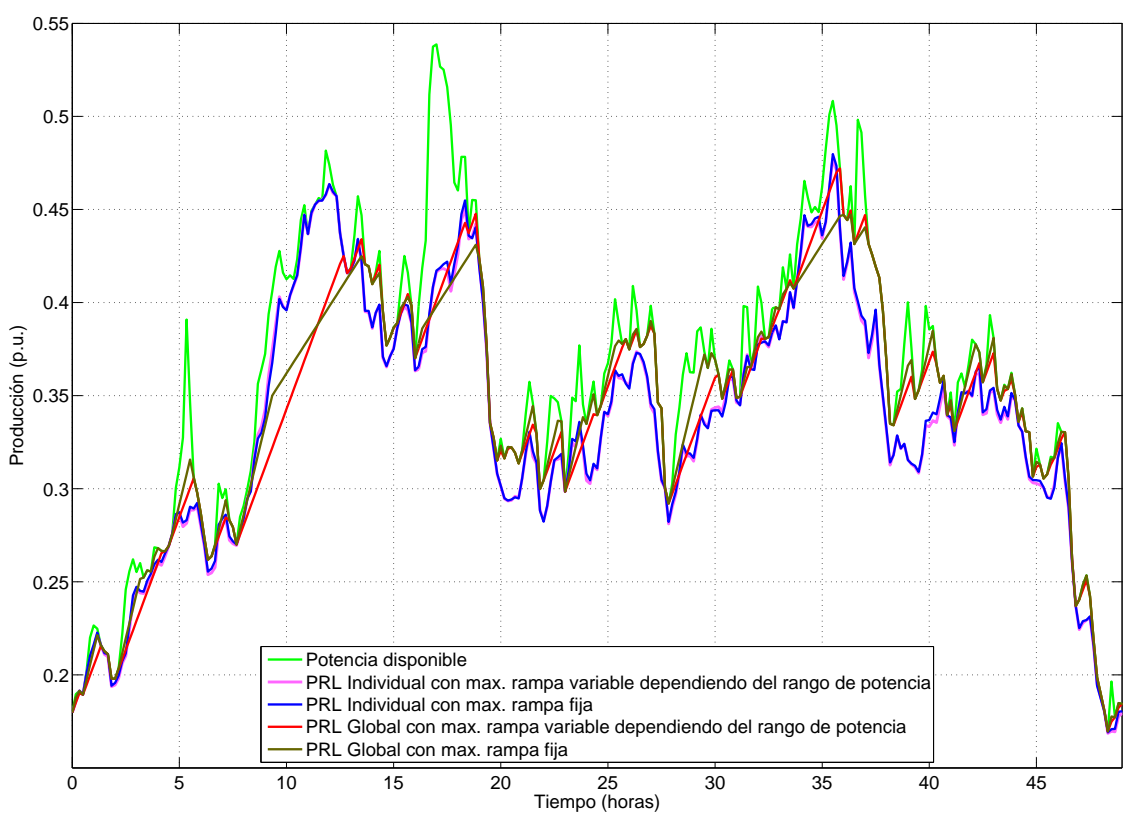

Figura 5.16: Comparativa de tipos de regulación y producción disponible (II)

métodos con una rampa positiva máxima fija. Los valores numéricos de esta relación aparecen en la tabla 5.1.

\subsubsection{PRL aplicado a los parques con mayores fluctuaciones}

De los parques estudiados existen algunos que debido a sus características y la meteorología existente en la zona producen unas fluctuaciones mucho mayores que otros, según se muestra en el apartado 3.3. Esto hace que la aplicación de la regulación sea más sencilla y más fácil de ejecutar. En el presente capitulo se aplica la regulación PRL a los parques con mayores fluctuaciones, manteniendo el resto de parques sin regulación y entregando toda la potencia disponible. Este método se describe en Martín-Martínez et al. (2009c). La aplicación se ha realizado de forma progresiva añadiendo a la regulación en cada caso el siguiente parque con los parámetros representativos de las fluctuaciones más pronunciados.

Para la agregación del grupo 1 de datos correspondiente a 9 parques durante 2007, se observa una mejora a bajas pérdidas regulando únicamente $66.6 \%$ de la potencia global que incluye los 6 parques con mayores fluctuaciones, mientras que según aumentan las perdidas, la regulación de todos los parques se ofrece como la mejor opción. Por otra parte, la mejora a bajas pérdidas también se presenta regulando un $39.4 \%$ de la potencia instalada total, incluyendo 4 parques, en la agregación con datos del grupo 2 correspondiente a 8 parques durante 2008. Sin embargo para pérdidas superiores 


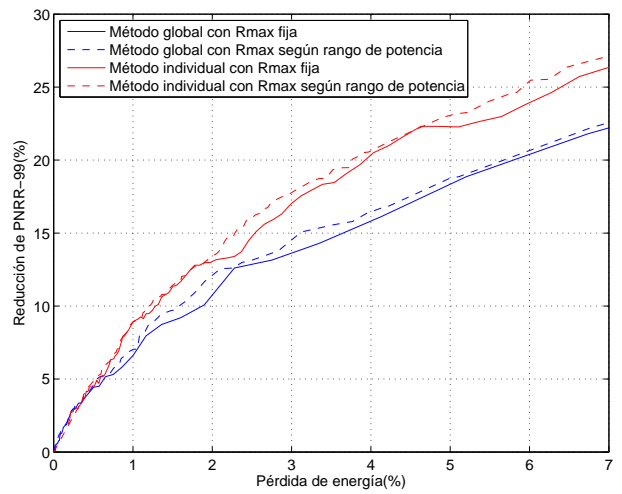

(a) Datos 2007

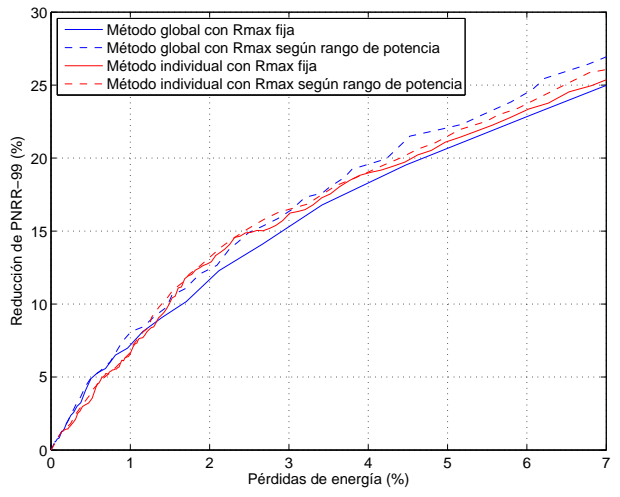

(b) Datos 2008

Figura 5.17: Reducción de $99 \%$ de rampa negativa y pérdidas para regulación PRL según rangos

Tabla 5.1: Regulación PRL aplicada según los rangos de producción. Valores de reducción porcentual de PNRR-99 dependiendo de las perdidas asociadas.

\begin{tabular}{|c|c|c|c|c|c|c|c|c|}
\hline \multirow{3}{*}{$\begin{array}{c} \\
\text { Pérdidas } \\
\text { de } \\
\text { energía }\end{array}$} & \multicolumn{2}{|c|}{ PRL Global } & \multicolumn{2}{|c|}{$\begin{array}{l}\text { PRL Global } \\
\text { según rango de } \\
\text { producción }\end{array}$} & \multicolumn{2}{|c|}{ PRL Individual } & \multicolumn{2}{|c|}{$\begin{array}{l}\text { PRL Individual } \\
\text { según rango de } \\
\text { producción }\end{array}$} \\
\hline & \multirow{2}{*}{\multicolumn{2}{|c|}{$\begin{array}{l}\text { Reducción } \\
\text { PNRR-99 } \\
(\%)\end{array}$}} & \multirow{2}{*}{\multicolumn{2}{|c|}{$\begin{array}{l}\text { Reducción } \\
\text { PNRR-99 (\%) }\end{array}$}} & \multirow{2}{*}{\multicolumn{2}{|c|}{$\begin{array}{l}\text { Reducción } \\
\text { PNRR-99( \%) }\end{array}$}} & \multirow{2}{*}{\multicolumn{2}{|c|}{$\begin{array}{l}\text { Reducción } \\
\text { PNRR-99 (\%) }\end{array}$}} \\
\hline & & & & & & & & \\
\hline & 2007 & 2008 & 2007 & 2008 & 2007 & 2008 & 2007 & 2008 \\
\hline $1 \%$ & 6.6 & 7.2 & 7.0 & 8.0 & 8.9 & 6.6 & 8.9 & 6.7 \\
\hline $2 \%$ & 10.7 & 11.7 & 12.1 & 12.4 & 13.0 & 12.8 & 13.4 & 13.2 \\
\hline $5 \%$ & 18.4 & 20.6 & 18.8 & 22.1 & 22.4 & 21.1 & 23.1 & 21.5 \\
\hline
\end{tabular}

al $1 \%$ la diferencia entre los resultados de la regulación del $100 \%$ y el resto de casos es considerable.

Se aprecia que se consiguen reducciones aceptables de PNRR-99 considerando solo una parte de los parques eólicos estudiados y dejando el resto a la máxima potencia disponible. Por ejemplo para el caso de 2007, con un $66 \%$ de la potencia total de los parques (WF $4+5+6+7+8+9$ ) y un $2 \%$ de pérdidas, la reducción del PNRR-99 es del $12.5 \%$. Otro ejemplo es aquel que en el caso de 2008 para un $35 \%$ del total de potencia, con unas pérdidas del 1 y $2 \%$, la reducciones del PNRR-99 son respectivamente 6.4 y $10.2 \%$. 


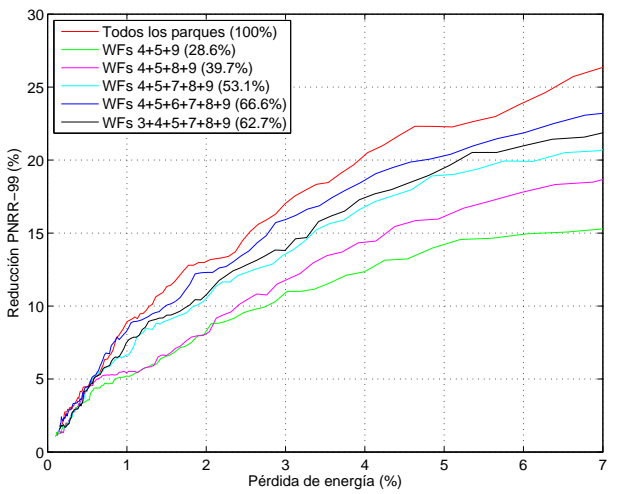

(a) Datos 2007

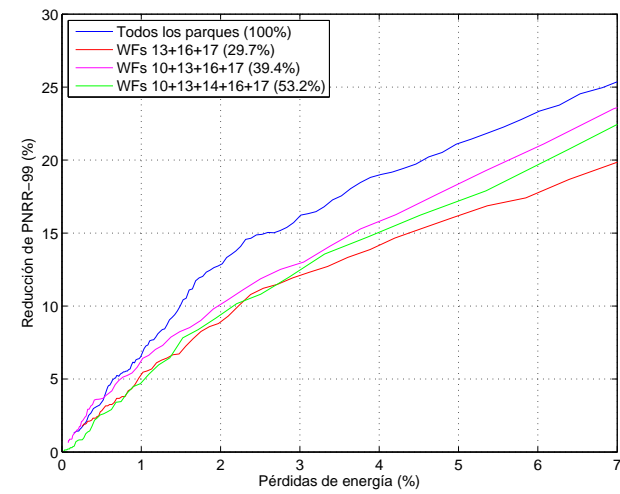

(b) Datos 2008

Figura 5.18: Reducción de $99 \%$ de rampa negativa con PRL aplicado a parques con mayores fluctuaciones

\subsubsection{PRL aplicado a la producción total de España}

En este apartado se aplica el PRL con una configuración global sobre la producción total agregada de la España Peninsular. El método es aplicado directamente sobre las series temporales de los años 2007, 2008, 2009, 2010, 2011 y 2012. Los resultados para la reducción de los parámetros PNRR-99 y desviación estándar se muestran en las figuras 5.19 y 5.20 respectivamente.

De los resultados se desprende que el nivel de reducción general conseguido es menor que en las aplicaciones anteriores. Esto es debido a que existe un mayor efecto de suavizado en las series temporales de la producción global española que en la serie temporal de la agregación de todos los parques para ambos años 2007 y 2008. Aunque, tal y como se comentó en el apartado 3.3, en la tabla 3.7 se aprecia que los valores de desviación estándar de las rampas y el valor PNRR-99 no disminuyen significativamente a partir de 2007 pese a que la potencia instalada se incrementó de 12015 MW a principios de 2007 hasta $22591 \mathrm{MW}$ a finales de 2012. Esto muestra la saturación del efecto de suavizado al alcanzar una cierta cantidad de potencia instalada en una región limitada. Como consecuencia las reducciones presentes no están relacionadas con la potencia total instalada y con el grado de agregación presente. Aún así, se obtienen reducciones importantes, como por ejemplo, para unas pérdidas del $2 \%$ la reducción de PNRR-99 ronda el rango de 4.4-6.8\%. Mientras que para la desviación estándar de las rampas con unas pérdidas del $2 \%$ la reducción está en el rango de 17.5-24.1\%, siendo ligeramente inferior a las reducciónes obtenidas en la figura 5.14 .

Para mejorar los resultados obtenidos en la aplicación del método PRL en estos ultimos apartados, la predicción eólica puede suponer una herramienta útil. Por lo tanto, a partir de la predicción eólica establecida, en lugar de 
Tabla 5.2: Regulación PRL aplicada a los parques con mayores fluctuaciones. Valores de reducción porcentual de PNRR-99 dependiendo de las pérdidas asociadas.

\begin{tabular}{llllll}
\hline & & $\begin{array}{l}\text { PRL con 1\% de } \\
\text { pérdidas }\end{array}$ & $\begin{array}{l}\text { PRL con 2\% de } \\
\text { pérdidas }\end{array}$ & $\begin{array}{l}\text { PRL con } 5 \% \text { de } \\
\text { pérdidas }\end{array}$ \\
\hline WF Regulados & $\begin{array}{l}\text { Porcentaje } \\
\text { controlado } \\
(\%)\end{array}$ & $\begin{array}{l}\text { Reducción } \\
\text { PNRR-99 }(\%)\end{array}$ & $\begin{array}{l}\text { Reducción } \\
\text { PNRR-99 }(\%)\end{array}$ & $\begin{array}{l}\text { de } \\
\text { Peducción } \\
\text { PNR-99 (\%) }\end{array}$ \\
\hline WF 4-5-9 & 28.6 & 5.3 & 8.1 & 14.2 \\
WF 4-5-8-9 & 39.7 & 5.9 & 8.3 & 16.1 \\
WF 4-5-7-8-9 & 53.1 & 6.7 & 10.6 & 19.0 \\
WF 4-5-6-7-8-9 & 66.6 & 8.3 & 12.3 & 20.3 \\
WF 3-4-5-7-8-9 & 62.7 & 7.8 & 10.8 & 19.5 \\
All 9 WFs & 100.0 & 8.9 & 13.0 & 22.4 \\
\hline WF 13-16-17 & 29.7 & 5.3 & 8.8 & 16.2 \\
WF 10-13-16-17 & 39.4 & 6.2 & 10.1 & 18.4 \\
WF 10-13-14-16-17 & 53.2 & 4.7 & 9.4 & 17.2 \\
All 8 WFs & 100.0 & 6.6 & 12.8 & 21.2 \\
\hline
\end{tabular}

aplicar el método PRL de forma constante se podría aplicar solo en ciertos eventos en los que se optimice su uso y por lo tanto las pérdidas derivadas sean mínimas.

\subsection{Regulación con constante Delta y limitación de rampa negativa (NRL)}

La regulación con constante Delta de producción consiste en la limitación de la producción actual a un valor de potencia fijado, denominado Delta, por debajo de la producción máxima admisible. Esta constante es usada normalmente para incrementar las capacidades de regulación de un parque eólico. La constante Delta se usa normalmente en conjunto con la limitación de rampa negativa (NRL), permitiendo también fijar una rampa negativa máxima para los casos en los que la velocidad de viento decrece y es posible limitar ese descenso en la producción reduciendo la constante Delta. Su uso se suele presentar considerando cualquier rampa negativa y por lo tanto ignorando esa limitación.

Esta regulación, con limitación mediante la constante Delta y sin regulación de rampa negativa, presenta unas pérdidas de energía prácticamente lineales como se puede observar en la figura 5.21. Por lo tanto las pérdidas registradas al utillizar esta regulación son proporcionales al valor de Delta utilizado.

En cuanto a su uso para la reducción de rampa negativa, los resultados indican una menor efectividad que la regulación PRL, como también se indica 


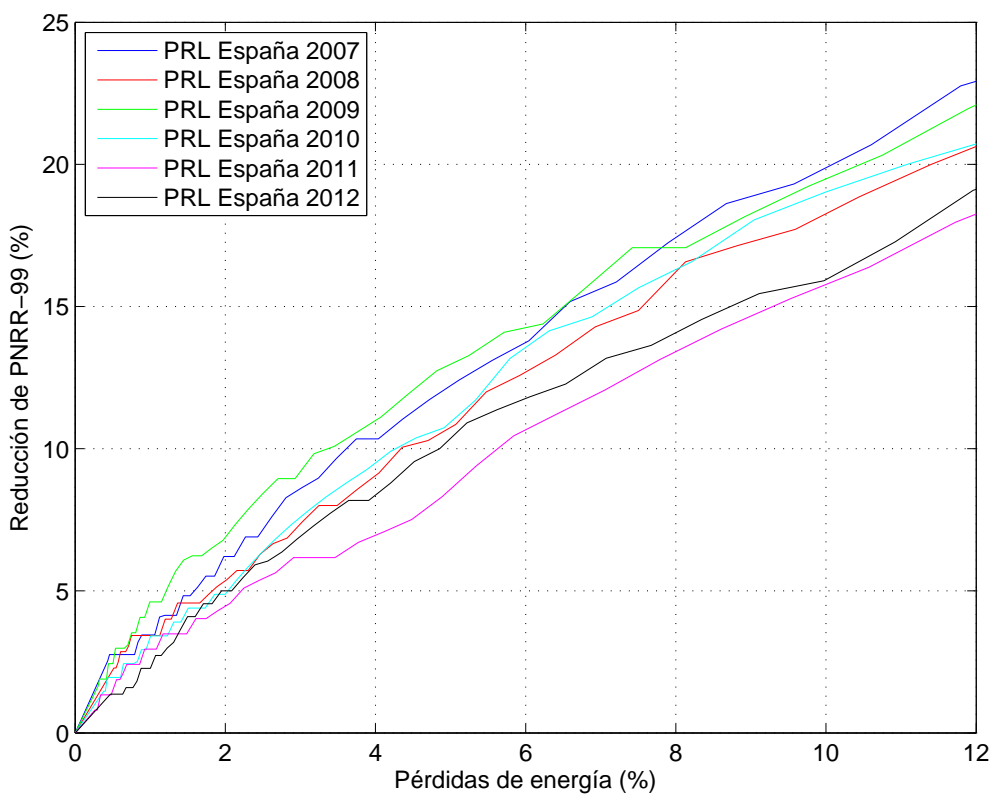

Figura 5.19: Reducción de $99 \%$ de rampa negativa con PRL aplicado a la producción total de España

en Martín-Martínez et al. (2009b), pero por contra se obtiene una mejor reducción global de las fluctuaciones cuando se aplica sobre toda la producción global bajo unas ciertas condiciones, como se estudió en Martín-Martínez et al. (2010a). Además su uso permite la contribución de la energía eólica como reserva a subir en el sistema, servicio del que no se podía disponer de forma continua en la regulación PRL.

Del mismo modo que en el método PRL se pueden utilizar dos configuraciones en el uso de la regulación Delta, el método global, aplicado sobre la serie temporal de la agregación y el método individual aplicado sobre cada parque de la agregación por separado.

En las figuras 5.22 y 5.23 se muestran los resultados de la aplicación del método Delta, tanto de forma global como individual a las series temporales estudiadas. Se muestran unas reducciones considerablemente superiores para el caso global. Aunque se corrobora que para la desviación y especialmente para el valor de PNRR-99 las reducciones obtenidas son claramente inferiores que las conseguidas con los diferentes métodos PRL.

\subsection{Control de tormenta (Storm Control)}

En esta sección se analiza el uso de una curva de potencia con control de tormenta sobre el parque eólico WF13 de forma que se obtenga una serie temporal resultante y se compare con la serie temporal original. En la figu- 


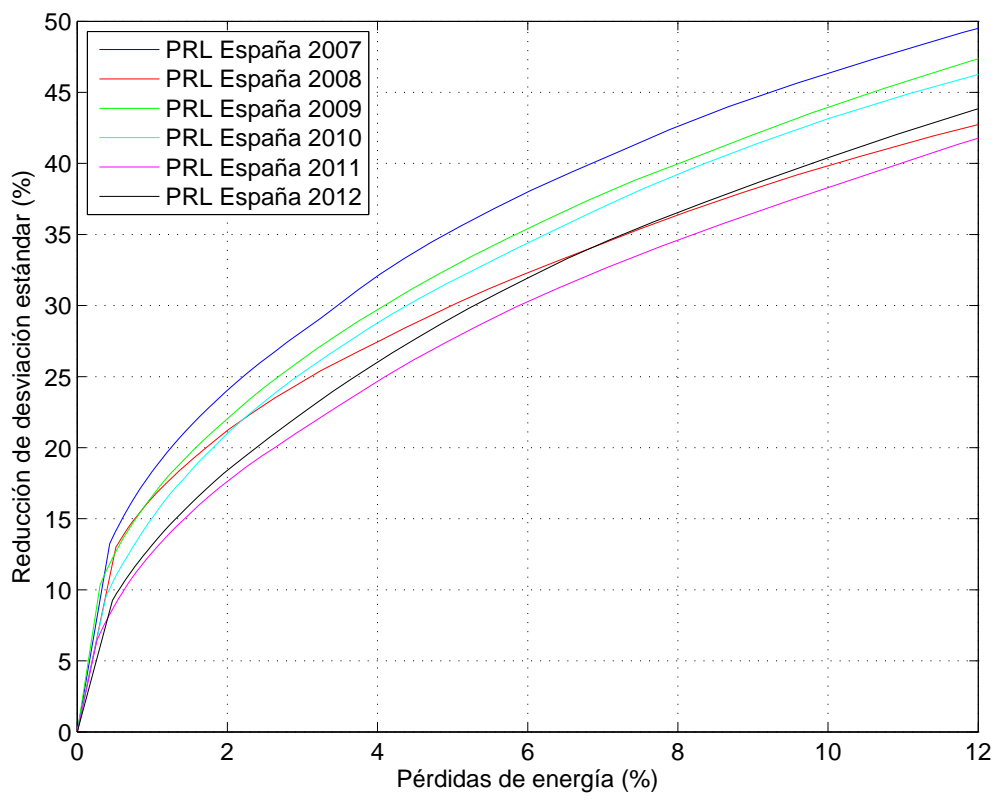

Figura 5.20: Reducción de desviación estándar con PRL aplicado a la producción total de España

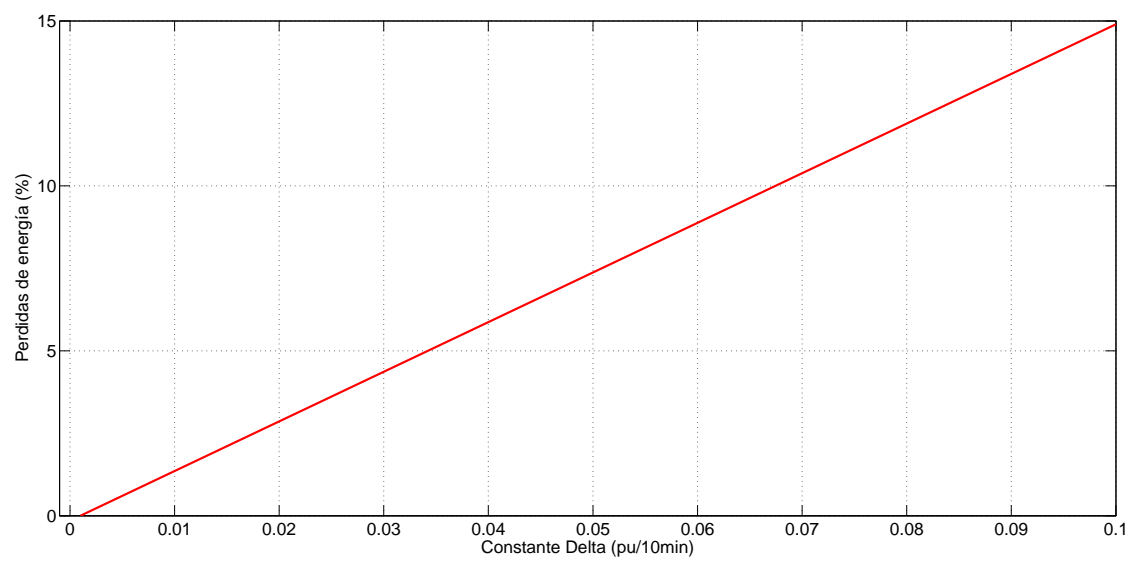

Figura 5.21: Pérdidas que resultan de la aplicación de la regulación de constante Delta

ra 5.24 se muestra el esquema utilizado. Para cada periodo de 10 minutos y para cada aerogenerador, si la velocidad de viento máxima es superior a $20 \mathrm{~m} / \mathrm{s}$, se establece una función de probabilidad lineal a partir de los datos de velocidad máxima, velocidad media y velocidad mínima. Estas probabilidades son aplicadas sobre la curva de potencia considerada obteniendo un valor de potencia generada resultante para el periodo considerado.

En las series temporales utilizadas, las cuales abarcan desde enero de 


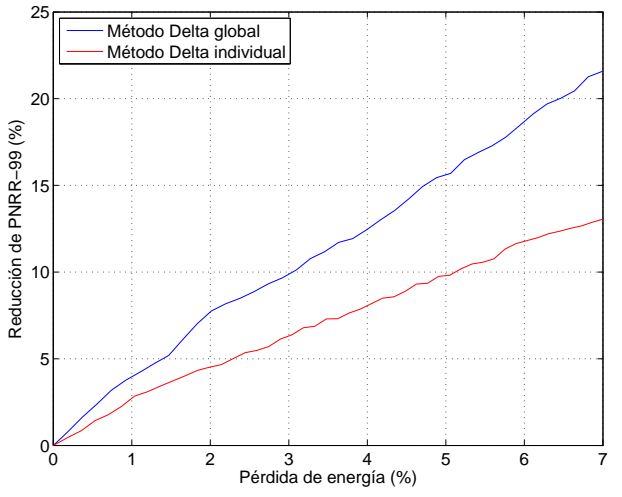

(a) Datos 2007

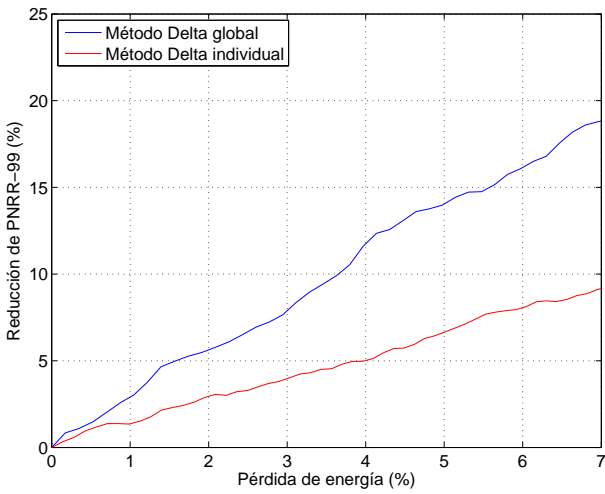

(b) Datos 2008

Figura 5.22: Reducción de PNRR-99 y pérdidas para regulación Delta individual y global

2007 hasta septiembre de 2010 existen 2811 periodos de 10 minutos en los que al menos un aerogenerador se encuentra expuesto a una velocidad de viento máxima superior a $20 \mathrm{~m} / \mathrm{s}$. Para este analisis se ha considerado una curva de potencia con un control de tormenta suave desde $20 \mathrm{~m} / \mathrm{s}$ hasta $25 \mathrm{~m} / \mathrm{s}$. En la figura 5.25 se indican la curva de potencia con control de tormenta utilizada y la curva de potencia original. Así mismo, se muestran los valores de potencia obtenidos como resultado de la aplicación del control de tormenta en comparación con los valores de potencia originales, según la velocidad media de cada periodo.

El resultado de la aplicación del control de tormenta se traduce en una reducción considerable de las rampas negativas mas extremas. Esto se puede apreciar en la figura 5.26 que muestra la comparativa entre la curva de duración de rampas original y la obtenida con la curva de potencia con control de tormenta suave de 20 a $25 \mathrm{~m} / \mathrm{s}$. El valor de PNRR-99 se reduce de 0.695 pu a 0.158 pu lo que supone un $77,3 \%$ de reducción. Por el contrario las rampas positivas apenas sufren cambios. 


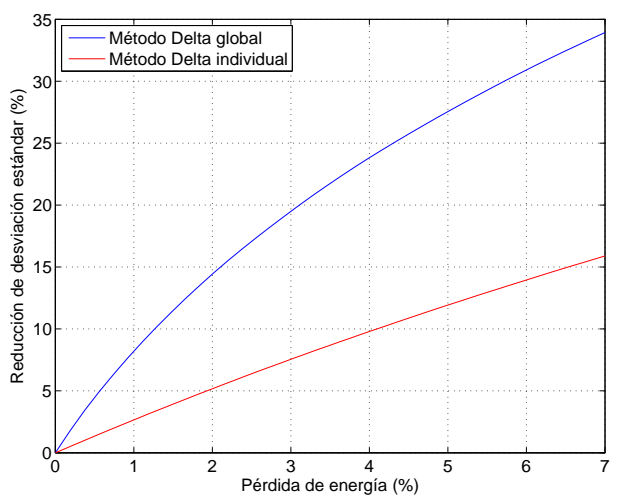

(a) Datos 2007

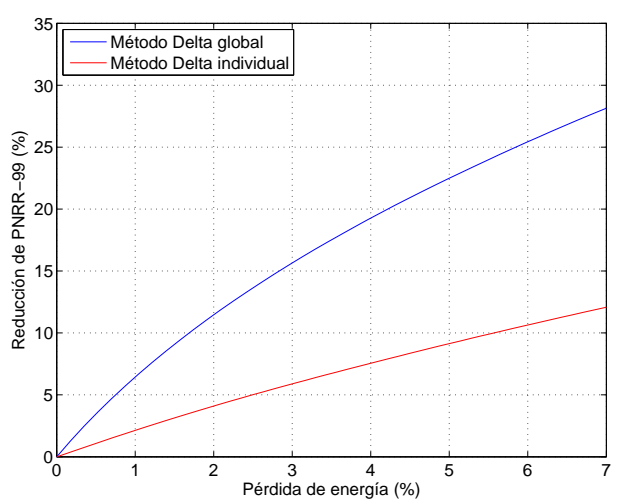

(b) Datos 2008

Figura 5.23: Reducción de la desviación estándar y pérdidas para regulación Delta individual y global 


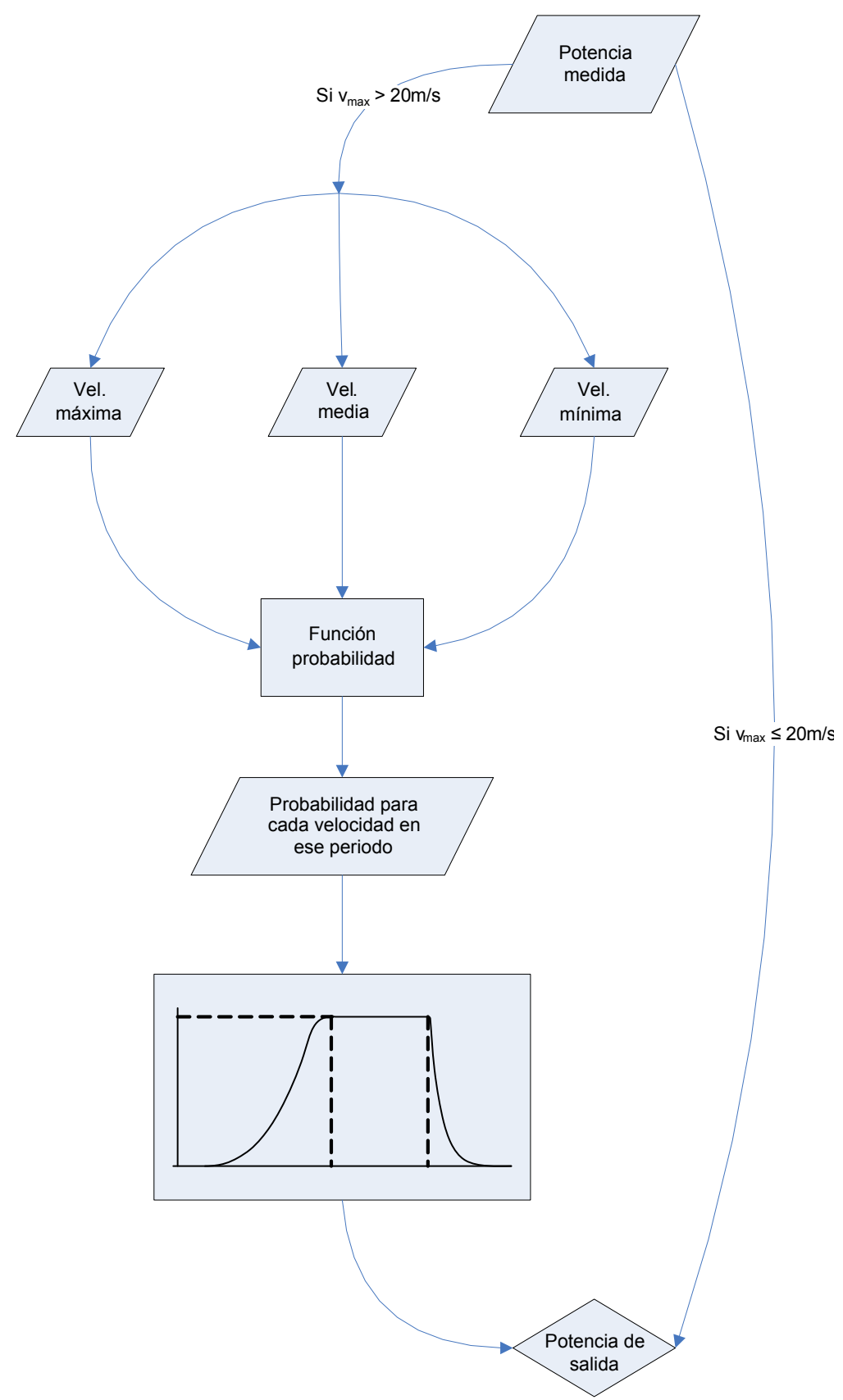

Figura 5.24: Esquema de la aplicación de una curva de potencia con control de tormenta sobre el parque WF13 de 2008 a 2010 


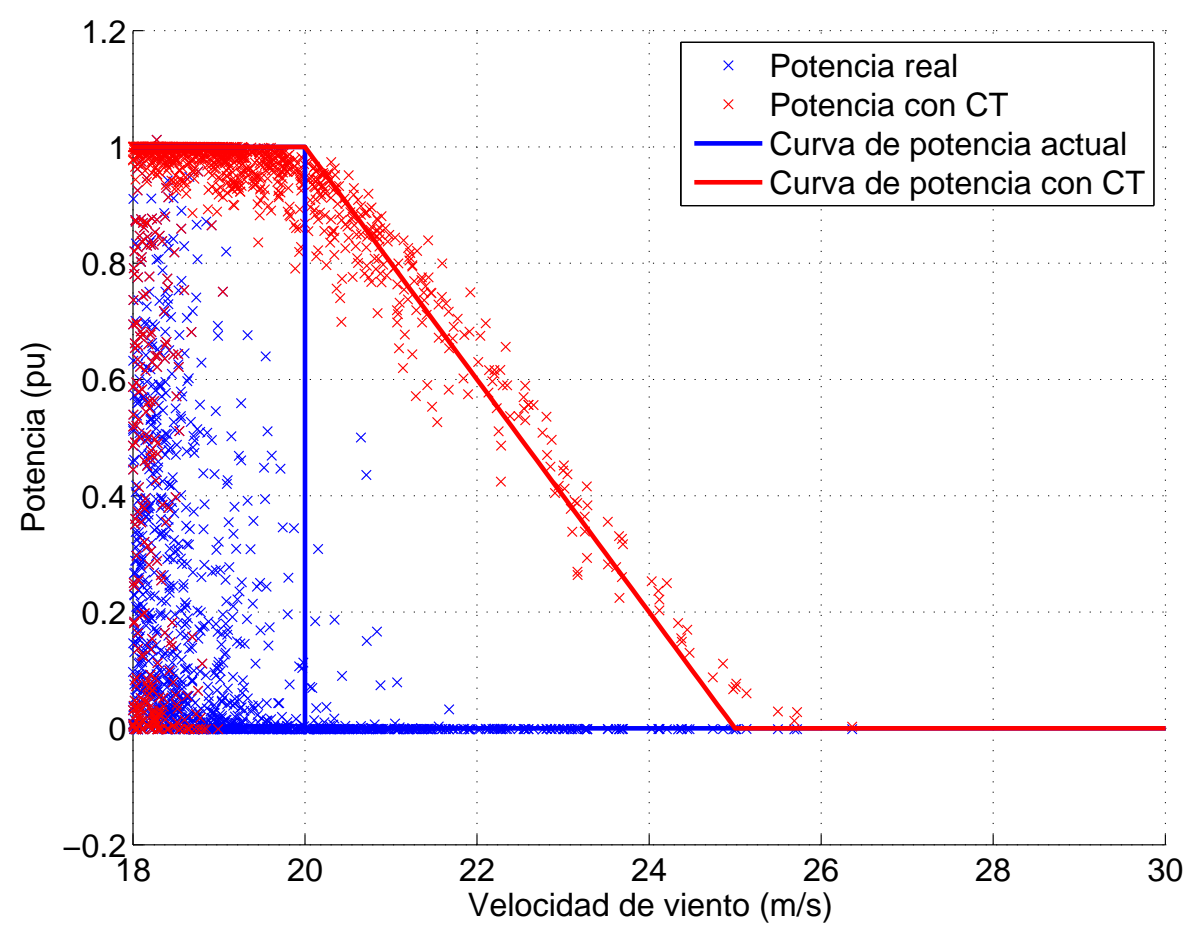

Figura 5.25: Comparación de valores reales de potencia en la zona de velocidad de corte con los conseguidos al aplicar una curva con control de tormenta sobre un aerogenerador del parque WF13 de 2007 a 2010 


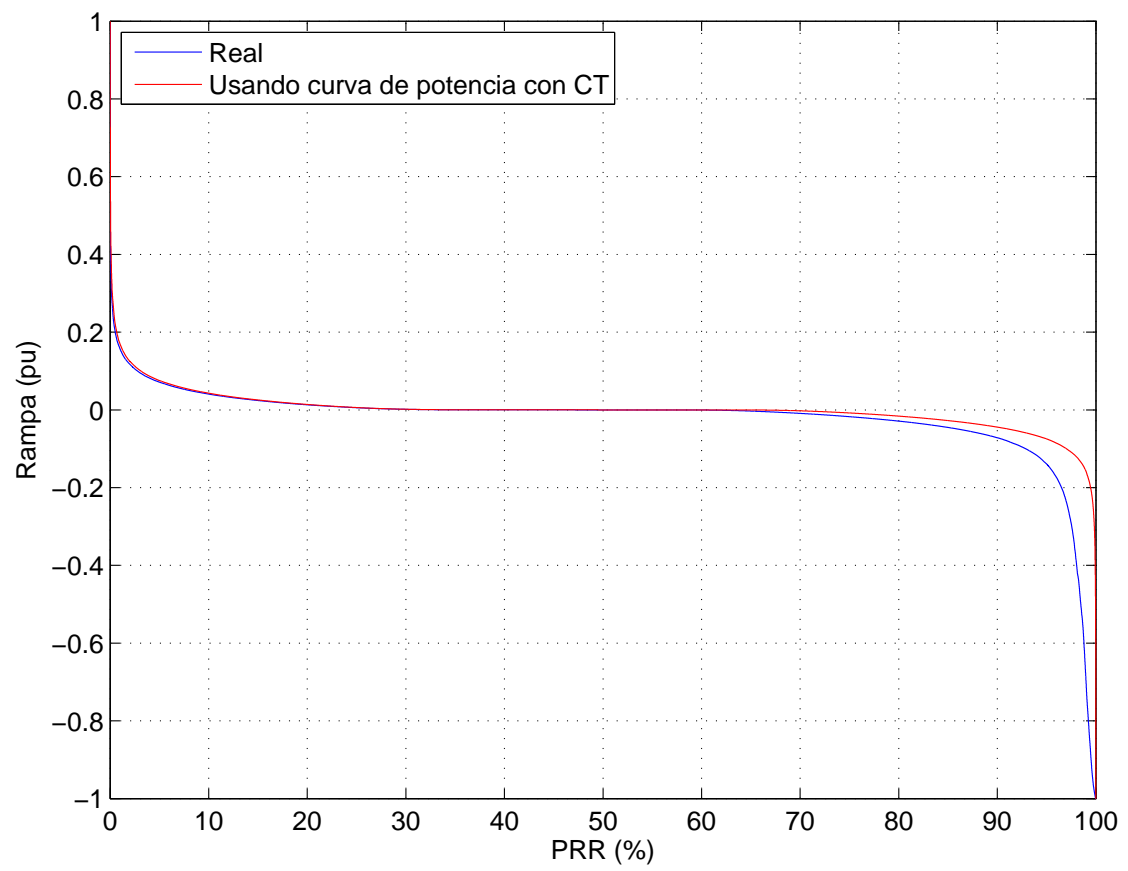

Figura 5.26: Reducción de los valores PRR al aplicar una curva con control de tormenta sobre el parque WF13 de 2008 a 2010 


\subsection{Conclusiones}

En el presente capitulo se han analizado diversos métodos con el objetivo de reducir las fluctuaciones de potencia eólica. Para llevar a cabo dicho análisis se ha usado el porcentaje de reducción de dos parámetros utilizados para caracterizar la variabilidad como son el PNRR-99 y la desviación estándar de las rampas.

En primer lugar se han analizado diversas configuraciones del método PRL. El PRL individual ofrece buenos resultados para la reducción de rampas negativas extremas, indicadas por el valor PNRR-99 mientras que para la reducción de la desviación estándar de las rampas el método PRL global es más adecuado. Al aplicar distintos valores de rampas máximas según el rango de potencia los porcentajes de reducción mejoran ligeramente tanto para PRL global como para PRL individual. Si solo se aplica el método PRL sobre los parques con mayores fluctuaciones se obtienen reducciones similares a las obtenidas en el PRL individual para pérdidas pequeñas, inferiores al 1\%, mientras que para perdidas superiores las reducciones son considerablemente inferiores.

Por otra parte, en el caso de la producción agregada total de la España peninsular, los resultados obtenidos con el método PRL indican menores reducciones de los parámetros PNRR-99 y desviación estándar de las rampas que las obtenidas con las agregaciones anteriores, debido al efecto de suavizado presente. Aún así, se obtienen reducciones importantes y las diferencias presentes entre los años estudiados no están relacionadas con la potencia total instalada, sino con la variabilidad inherente del viento, debido a la saturación del efecto de suavizado.

En el caso de la constante Delta, los resultados obtenidos son inferiores a los obtenidos con el método PRL, especialmente en la reducción del valor PNRR-99. Sin embargo el uso de este método puede aportar una cierta cantidad de potencia a subir en caso que sea necesaria en la regulación del sistema. Esto permite a la energía eólica contribuir a las reservas del sistema.

Por último, el control de tormenta presenta una gran reducción de las rampas negativas más extremas aportando además una cantidad adicional de energía generada durante periodos de alta velocidad de viento. Esta reducción de las rampas negativas puede permitir una entrada escalonada de las reservas en caso de tormentas que provoquen la protección por sobrevelocidad en los parques eólicos de una zona. 


\title{
Capítulo 6
}

\section{Conclusiones, aportaciones y trabajos futuros}

\begin{abstract}
RESUMEN:
Se presenta el último capítulo de la Tesis Doctoral con el objeto de reunir las conclusiones generales obtenidas en los capítulos 2, 3, 4 y 5 . Además, se resumen las principales aportaciones, junto con las publicaciones derivadas, y se proponen sugerencias para trabajos futuros.
\end{abstract}

\subsection{Conclusiones}

El aumento progresivo de la potencia eólica instalada en los sistemas de energía eléctrica genera la necesidad de evaluar la integración de este tipo de energía desde diferentes puntos de vista. La presente Tesis Doctoral ha analizado, desde una base, las fluctuaciones de potencia eólica, sus efectos sobre el sistema eléctrico y diversos métodos para atenuar dichas fluctuaciones, alcanzando las conclusiones que se indican a continuación.

Tras evaluar las características y los parámetros de las fluctuaciones de potencia eólica se ha comprobado que los modelos espectrales de velocidad de viento son ampliamente utilizados en el campo de la energía eólica. Dichos modelos suelen basarse, por un lado, en una función de densidad de potencia espectral que explica las variaciones de la velocidad del viento en un punto, y por otro, en una función de coherencia espectral que contiene la relación entre las oscilaciones de la velocidad del viento en distintos puntos.

Los modelos de PSD y de coherencia espectral para análisis de fluctuaciones disponibles actualmente en la bibliografía se centran principalmente en los parques eólicos marinos, en zonas de costa o en emplazamientos terrestre con características similares al mar. Por ello, apenas consideran el efecto de la orografía y se ha percibido una carencia de modelos de parques eólicos terrestres en el que se incluya características de terreno complejo. 
Adicionalmente, se ha seleccionado el valor PNRR-99 como un parámetro adecuado y conservador a la hora de caracterizar los requerimientos de reservas asociados con la potencia eólica. Se ha estudiado la dependencia entre el valor PNRR-99 y el nivel de potencia del parque eólico o la agregación correspondiente. Los resultados muestran valores PNRR-99 más negativos a cargas parciales para parques individuales y a altas cargas para grandes agregaciones.

Por otra parte, en el sistema eléctrico español la energía eólica tiene un peso prominente y ante la gran capacidad instalada se ha observado que se ha alcanzado un alto grado de saturación del efecto de suavizado en los últimos años. Esto indica que los parámetros relativos de la variabilidad de la potencia eólica tenderán a mantenerse constantes aunque se aumente la potencia instalada en los próximos años.

Los requerimientos de reservas deben ser incrementados para conseguir una fiabilidad adecuada cuando el nivel de potencia eólica presente aumenta. También se han estudiado los errores de predicción eólica, observándose un alto grado de error de predicción eólica en cierto tipo de eventos lo que conlleva la necesidad de introducir sistemas de regulación de la potencia activa de origen eólico para mantener al sistema en condiciones de estabilidad y fiabilidad y para reducir la cantidad y el coste de las reservas.

Finalmente, se han estudiado estrategias de regulación, como son el PRL y el control Delta. Los resultados indican unas reducciones considerables del valor de PNRR-99 con unas perdidas asumibles 1\%-2\%. La configuración que mejores reducciones obtiene es el PRL individual con rampa máxima $R_{\max }$ dependiente del nivel de carga. Aunque el PRL global ofrece mejores reducciones para valores de rampas menos extremas como PNRR-90. El control Delta también obtiene unos resultados aceptables y tiene la ventaja añadida de ofrecer la posibilidad de aportar reservas primarias a subir al generar una potencia Delta por debajo del máximo producible. Estos métodos pueden ser incluidos en un sistema automático de control de la generación (AGC) y podrían ser optimizados usando la predicción de potencia eólica. De forma similar, el método de estimación de frecuencia estudiado en el apéndice A puede ser utilizado en conjunto con los métodos desarrollados en pudiendose establecer los parámetros de $R_{\max }$ y Delta que se deben usar para mantener el sistema en condiciones óptimas de funcionamiento a partir de la energía primaria cedida por las instalaciones eólicas.

\subsection{Aportaciones}

Las principales aportaciones novedosas y originales de la presente Tesis Doctoral se pueden resumir en las siguientes líneas:

- Propuesta del uso del valor PNRR-99 para caracterizar las rampas 
de potencia eólica de forma que se pueda utilizar para calcular los requerimientos de reservas.

- Análisis del efecto de suavizado para varias agregaciones de parques eólicas y para la producción global de España.

- Evaluación las fluctuaciones eólicas presentes en el sistema eléctrico español, incluyendo sus efectos, su predicción y eventos extremos registrados.

- Análisis y validación del uso de la regulación PRL y el control Delta para la reducción de la variabilidad de la energía eólica.

- Determinación de los niveles de reducción de la variabilidad de la potencia eólica usando la regulación PRL y el control Delta y determinación de los casos en los que se optimiza el uso de las mismas.

- Desarrollo de un método DPLL para la estimación instantánea de la frecuencia de red bajo perturbaciones equilibradas y desequilibradas.

En línea con el trabajo desarrollado se han realizado las siguientes publicaciones:

- Martín-Martínez, S.; Gómez-Lázaro, E.; Molina-García, A.; Fuentes, J. A.; Vigueras-Rodríguez, A. y Amat Plata, S. "A new three-phase dpll frequency estimator based on nonlinear weighted mean for power system disturbances". Power Delivery, IEEE Transactions on, vol. 28, páginas 179-187, 2013.

- Martín-Martínez, S.; Vigueras-Rodríguez, A.; Gómez-Lázaro, E.; Fuentes, J. A. and Molina-García A. "Analysis of Positive Ramp Limitation control strategies for reducing wind power fluctuations", aceptado para publicación en la revista IET Renewable Power Generation, April 2013.

- Martín-Martínez, S.; Vigueras-Rodríguez, A.; Gómez-Lázaro, E.; MolinaGarcía, A.; Muljadi, E. y Milligan, M. "Advances in wind power", capítulo "Wind Power Variability and Singular Events", páginas 285-304. InTech, 2012.

Así como contribuciones a congresos:

- Vigueras-Rodríguez, A.; Sørensen, P.; Cutululis, N.; Viedma, A.; GómezLázaro, E. and Martín-Martínez, S. "Application of ramp limitation regulations for smoothing the power fluctuations from offshore wind farms", in European Wind Energy Conference and Exhibition (EWEC09), Marseille (France), March 2009. 
- Martín-Martínez, S., Gómez-Lázaro, E., Vigueras-Rodríguez, A., FuentesMoreno, J. A. y Molina-García, A. "Regulation strategies for wind power fluctuations depending on demand (in Spain)". En 8th International Workshop on Large-Scale Integration of Wind Power. Quebec (Canada), 2010a.

- Martín-Martínez, S., Gómez-Lázaro, E., Vigueras-Rodríguez, A., FuentesMoreno, J. A. y Molina-García, A. "Regulation strategies for wind power fluctuations in a system depeding on ramping power range". En European Wind Energy Conference and Exhibition (EWEC2010). Warsaw (Poland), 2010b.

- Martín-Martínez, S., Gómez-Lázaro, E., Vigueras-Rodríguez, A., MolinaGarcía, A., Milligan, M. y Muljadi, E. "Participation of wind power plants in the spanish power system during events". En IEEE PES General Meeting, San Diego, USA, página 8. 2012.

- Martín-Martínez, S., Gómez-Lázaro, E., Vigueras-Rodríguez, A., FuentesMoreno, J. A. y Molina-García, A. "Reducing wind power fluctuations in a system by applying ramp limitations to wind farms with greater oscillations". En Nordic Wind Power Conference. Bornholm, Denmark, 2009c. ISBN 978-87-92465-06-1.

- Martín-Martínez, S., Gómez-Lázaro, E. y Vigueras-Rodríguez, A. "Regulation Technique for Smoothing Active Power in Aggregated Wind Farms distributed within Spain". En 8th International Workshop on Large Scale Integration of Wind Power. Bremen (Germany), 2009.

- Martín-Martínez, S., Gómez-Lázaro, E. y Vigueras-Rodríguez, A. "Comparison of power fluctuations from onshore and offshore wind farms". En IEA Task 23 Back-to-Back Workshop: Offshore wind farms - wake effects and power fluctuations. Roskilde, Denmark, 2009a.

\subsection{Trabajos futuros}

En virtud de las conclusiones obtenidas en la Tesis Doctoral, se pueden sugerir algunas nuevas líneas de investigación como posibles trabajos futuros dentro de este campo se indican los siguientes:

- La optimización de la regulaciones PRL y constante Delta estableciendo los periodos para un aplicación óptima y los periodos de no utilización. También se puede ejecutar una regulación en la que se usen ambas de manera alternativa.

- Establecer la correlación entre la producción de varios parques para optimizar y reducir la variabilidad de su producción conjunta. 
- Establecer modelos agregados de red con componentes eólicos, térmicos e hidráulicos para determinar el funcionamiento conjunto y disminuir las pérdidas de ciclo.

- Estudiar la regulación individual de los aerogeneradores de manera que la producción del parque sea lo más parecida a una consigna indicada.

- Evaluar modelos espectrales en emplazamientos terrestres con orografía compleja, determinando la necesidad de modificación de los mismos en función de la complejidad del terreno. Para caracterizar la orografía del emplazamiento se propone la utilización del parámetro RIX ("Roughness IndeX").

- Optimizar el uso de los métodos de control de potencia activa de modo que en conjunto con el método de estimación de frecuencia DPLL estudiado se puedan establecer los parámetros de $R_{\max }$ y Delta para realizar el control de la energía primaria cedida por las instalaciones eólicas en caso de necesidades de reservas. 



\title{
Apéndice A
}

\section{Estimación de la frecuencia de red mediante un DPLL trifásico y media no lineal}

\begin{abstract}
RESUMen: En este apéndice se propone un método trifásico digital de Lazo de seguimiento de fase (DPLL) para determinar la frecuencia instantánea de red bajo perturbaciones en sistema eléctrico. La solución propuesta se basa en algoritmos monofásicos DPLL definiendo la frecuencia de red estimada como una media no lineal ponderada de las salidas de tres DPLL monofásicos. Las señales ortogonales requeridas por los algoritmos DPLL se obtienen a través de la tensión de cuadratura ficticia usando 5 métodos apropiados diferentes. Las estructuras propuestas han sido llevadas a cabo a través de un análisis exhaustivo en eventos equilibrados y desequilibrados. Es por ello que se han utilizado huecos de tensión reales, extraídos de las mediciones en un parque eólico español durante varios meses, como ejemplos para estimar la frecuencia de red. Por ultimo, los resultados obtenidos de las topologías DPLL trifásicas se comparan para confirmar la validez de las estructuras DPLL propuestas.
\end{abstract}

\section{A.1. Introducción}

La frecuencia es considerada como uno de los parámetros más importantes en los sistemas eléctricos de potencia, siendo usada generalmente para indicar el estado de operación del sistema, como se señala en Huang et al. (2008). En este contexto, los desequilibrios entre demanda y generación normalmente causan desviaciones de la frecuencia del sistema sobre su valor nominal, produciendo situaciones de subfrecuencia y sobrefrecuencia y siendo necesario aportar las protecciones adecuadas bajo esas condiciones anormales. Anteriormente a 1979, las medidas de frecuencia se basaban en 
ApÉNDICE A. Estimación de la frecuencia de red mediante un DPLL 154 trifásico y media no lineal

dispositivos electromecánicos y analógicos como indica Sachdev (1997). Mas tarde, y con la utilización de los microprocesadores, el uso del control digital y la protección de los sistemas de potencia han demandado, más y más, la estimación de la frecuencia y su variación en tiempo real. De hecho, la velocidad en la estimación de la frecuencia ha llegado a ser extremadamente importante en la mayoría de las aplicaciones, siendo necesario encontrar un compromiso entre la velocidad de la estimación y su precisión. Akke en Akke (1997) especifica el criterio que una estimación de frecuencia adecuada debe satisfacer: una rápida velocidad de convergencia, precisión en la estimación de la frecuencia, y robustez frente al ruido. Aunque, es necesario señalar que debido a los cortos periodos de tiempo de observación, cualquier cambio por impulso de la señal de entrada puede producir un gran cambio en la frecuencia estimada. Estos cambios no deben ser considerados como cambios representativos en la velocidad rotacional de la planta, y no deben ser tomados en consideración para propósitos de control o protección. Por esta razón, como indica Moore et al. (1996), la susceptibilidad de ruido normalmente requiere una cuidadosa consideración de los requerimientos de filtrado

Un gran número de métodos para la estimación de frecuencia han sido propuestos a lo largo de las últimas décadas. Por ejemplo, las técnicas convencionales de Paso por Cero (Zero Crossing) en Kumar et al. (1975), Paso por Nivel (Level Cross) en Nguyen y Srinivasan (1984), Error de mínimos cuadrados (LES) en Sachdev y Giray (1985), LES recursivo en Sachdev y Nagpal (1991) o el filtro Kalman en Kamwa y Srinivasan (1993). En Duric et al. (1994), la componente de corriente continua y los armónicos son omitidos de ambas señales, tensión y corriente, proponiendo un método NewtonRaphson iterativo. El procesado de la forma de onda de la tensión incluyendo los armónicos y el ruido se presenta en Dash et al. (1997) mediante el uso de redes neuronales adaptativas. En Szafran y Rebizant (1998), dos filtros ortogonales de respuesta de impulso finito (FIR) son presentados, donde un algoritmo basado en las medidas de los componentes de la señal ortogonal se muestran. Se utilizan diferencias finitas en lugar de derivativas, requiriendo un menor periodo de muestreo en comparación con las referencias anteriores. La Transformada Discreta de Fourier, en Lobos y Rezmer (1997), y la Transformada Discreta de Fourier Smart basada en algoritmos numéricos han sido también propuestas para determinar las desviaciones de la frecuencia, como se describe en Yang y Liu (2001). Aunque, estos algoritmos requieren amplias ventanas de medida para oscilaciones pequeñas de la frecuencia, como indica Karimi-Ghartemani y Iravani (2003). En Terzija (2003), un nuevo y mejorado algoritmo numérico recursivo de tipo Newton es descrito, particularmente adecuado para fasores, armónicos, constantes de tiempo, y medidas locales de frecuencia. Por este camino, un estudio comparativo, realizado en Djuric y Djurisic (2008), muestra que los algoritmos basados en el método Newton eran menos sensitivos a la componente continua y a las distor- 
siones armónicas, pero con menores necesidades de hardware. En Salcic et al. (2009), un nuevo algoritmo de medida de frecuencia basado en el método Taylor adecuado para una implementación digital (ITAM) ha sido evaluado. Recientemente, un algoritmo recursivo de Transformada Wavelet en tiempo real ha sido propuesto por Ren y Kezunovic (2011).

Los métodos de estimación de la frecuencia basados en técnicas de Lazo de seguimiento de fase (PLL) y Lazo de seguimiento de fase digital (DPLL) también han sido utilizadas como un medio común de conocer tanto los valores del ángulo de fase como de la frecuencia en los sistemas eléctricos de potencia, como por ejemplo en Razabi (1996). Para aplicaciones monofásicas, algunas contribuciones están enfocadas en detectar las diferencias del ángulo de fase y por tanto en la estimación de la frecuencia. Algunos ejemplos de este tipo de aplicaciones son Sakamoto et al. (2002); Song et al. (2002); Silva et al. (2004); Karimi et al. (2004). Estas soluciones han sido normalmente propuestas para el control de convertidores electrónicos de potencia. Aunque, para aplicaciones de red, como indica Freijedo et al. (2009), en general, los PLLs monofásicos son más complejos que el PLL trifásico con cuadro síncrono de referencia (SRF). Adicionalmente, los PLLs monofásicos están limitados por la presencia de ondas de frecuencia doble que producen un respuesta errónea del método, como indica Jovcic (2003). Por otro lado, algunos autores afirman que suele ser más difícil reconocer el comportamiento exacto de los algoritmos PLL y comparar su funcionamiento debido a que los resultados no se presentan de manera satisfactoria: normalmente en forma de dientes de sierra o senos que representan los ángulos de fase reales y estimados Filho et al. (2008).

Con el objetivo de superar este inconveniente, este capítulo propone un método que contribuye en el campo de la estimación de la frecuencia instantánea con soluciones basadas en diferentes topologías de DPLL monofásicos y aplicando una estimación no lineal propuesta por los autores del articulo Martín-Martínez et al. (2013). Estas diferentes topologías para sistemas de red trifásicos han sido comparados y llevados a cabo bajo perturbaciones reales recogidas en un parque eólico español a lo largo de varios meses. De forma adicional, los resultados de dicha aplicación y del uso de algoritmos DPLL trifásicos convencionales son comparados.

\section{A.2. Estructuras DPPL}

Atendiendo a los diferentes algoritmos PLL propuestos en la literatura específica de este campo, se puede afirmar que los métodos PLL están formados normalmente por una estructura estándar compuesta por un Detector de fase (PD) que es no lineal generalmente, un Filtro de bucle (LF), y un Oscilador controlado por tensión (VCO), tal y como indica Freijedo et al. (2009). 
ApÉNDICE A. Estimación de la frecuencia de red mediante un DPLL

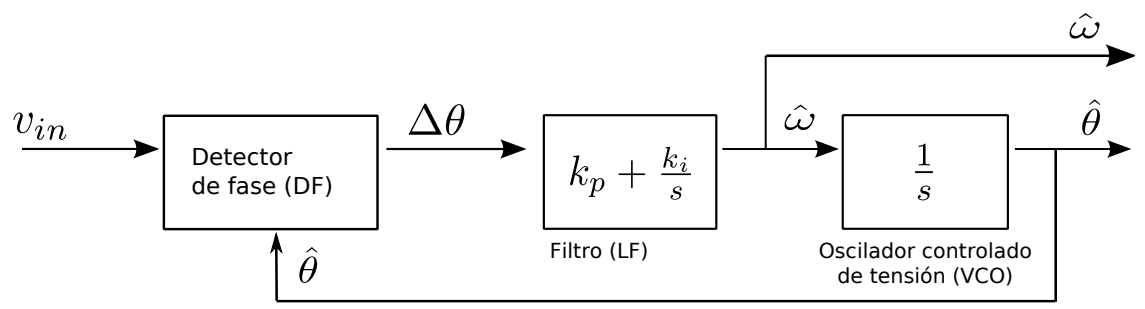

Figura A.1: Estructura PLL. Implementación de las etapas LF y VCO.

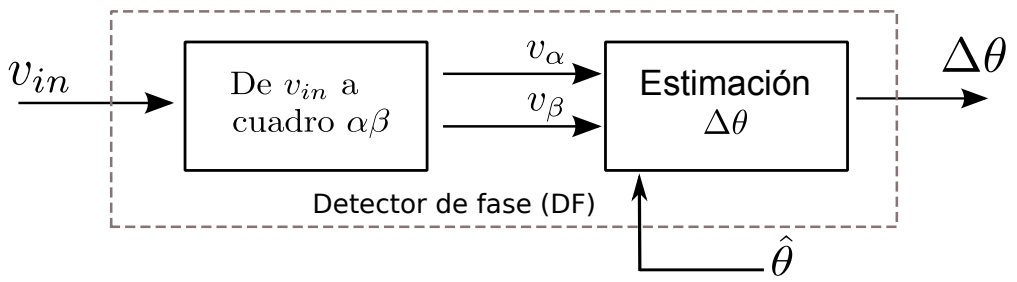

Figura A.2: Detector de fase (PD): Esquema general.

La implementación de las etapas de LF y VCO es común en la mayoría de las soluciones publicadas en la literatura: un controlador ProporcionalIntegral (PI) para cumplir los requerimientos del control en lazo cerrado basados en la comparación del ángulo de fase estimado y el ángulo de fase de la señal de entrada, según indica Karimi-Ghartemani (2006); y por otro lado un integrador representando la función del VCO como propone Filho et al. (2008), y se puede apreciar en la figura A.1.

Una arquitectura usada habitualmente para la etapa PD se basa en la división del proceso en dos partes: la primera se centra en la estimación del vector espacial de la tensión con los componentes $v_{\alpha}$ y $v_{\beta}$; la segunda se centra en determinar el error del ángulo de fase $(\Delta \theta)$, como muestra la figura A.2.

\section{A.2.1. Estimación del Vector Espacial de la Tensión}

Para señales de entrada trifásicas, las componentes $v_{\alpha}$ y $v_{\beta}$ son determinadas a través de la transformada lineal de Clarke, ver figura A.3a y ecuación A.1, como indica Chung (2000); Cataliotti et al. (2007).

$$
\left[\begin{array}{l}
v_{\alpha} \\
v_{\beta}
\end{array}\right]=\left[T_{s}\right]\left[\begin{array}{l}
v_{a} \\
v_{b} \\
v_{c}
\end{array}\right]=\frac{2}{3}\left[\begin{array}{ccc}
1 & -\frac{1}{2} & -\frac{1}{2} \\
0 & -\frac{\sqrt{3}}{2} & \frac{\sqrt{3}}{2}
\end{array}\right]\left[\begin{array}{l}
v_{a} \\
v_{b} \\
v_{c}
\end{array}\right]
$$

Asumiendo un sistema de potencia trifásico equilibrado donde $v_{a}=$ $V \cos (\omega t)$; las componentes $v_{\alpha}$ y $v_{\beta}$ son entonces $v_{\alpha}=V \cos (\omega t)$ y $v_{\beta}=$ $V \sin (\omega t)$ respectívamente. 


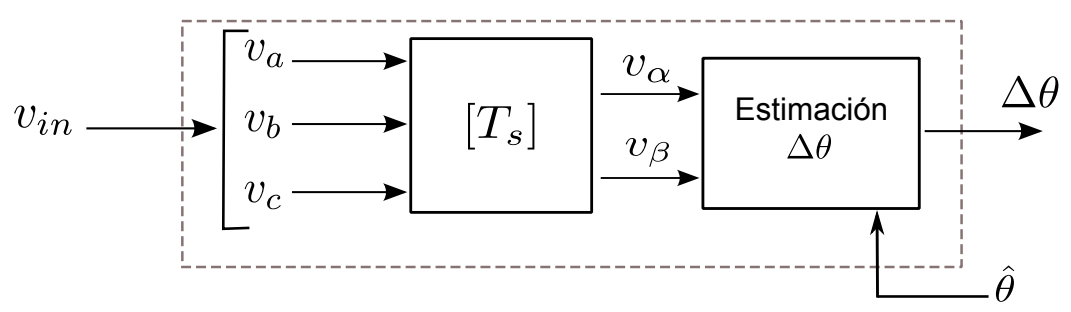

(a) Señal de entrada trifásica: Transformación lineal de Clarke

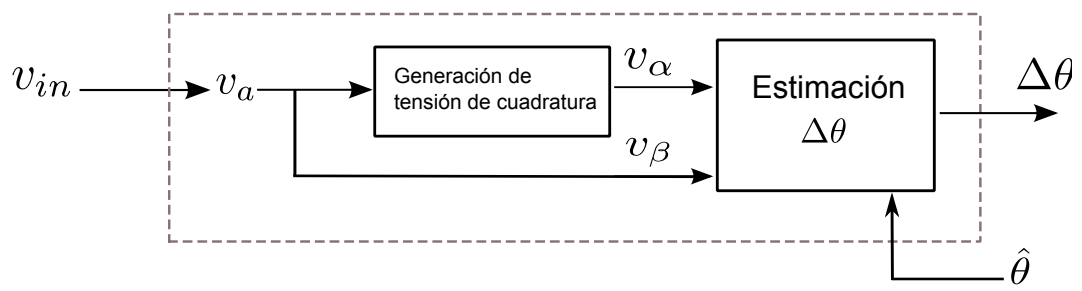

(b) Señal de entrada monofásica: Generación de la tensión de cuadratura

Figura A.3: Diagrama de bloques del cuadro $\alpha-\beta$

Tabla A.1: Estructuras de detector de fase (PD): Configuraciones propuestas para señales de entrada monofásicas.

\begin{tabular}{|c|c|c|}
\hline $\begin{array}{ll}\text { Método } & \text { de } \\
\text { cuadro } \alpha \beta & \end{array}$ & Método de estimación de $\Delta \theta$ & Nomenclatura \\
\hline \multirow{2}{*}{ Tabla de Memoria } & Función arcotangente & MeTa-ArcTg \\
\hline & Cuadro de referencia síncrono & $\mathrm{MeTa}-\mathrm{SRF}$ \\
\hline \multirow{2}{*}{$\begin{array}{l}\text { Amplitud y fase } \\
\text { estimadas }\end{array}$} & Función arcotangente & $\mathrm{EPhA}-\mathrm{AcTg}$ \\
\hline & Cuadro de referencia síncrono & $\mathrm{EPhA}-\mathrm{SRF}$ \\
\hline \multirow{2}{*}{ Filtro de $1^{\circ}$ orden } & Función arcotangente & $1 \mathrm{OrF}-\mathrm{AcTg}$ \\
\hline & Cuadro de referencia síncrono & 1OrF-SRF \\
\hline \multirow{2}{*}{ Filtro de $2^{\circ}$ orden } & Función arcotangente & $2 \mathrm{OrF}-\mathrm{AcTg}$ \\
\hline & Cuadro de referencia síncrono & $2 \mathrm{OrF}-\mathrm{SRF}$ \\
\hline \multirow{2}{*}{ Filtro pasa-todo } & Función arcotangente & $\mathrm{AllF}-\mathrm{AcTg}$ \\
\hline & Cuadro de referencia síncrono & AllF-SRF \\
\hline
\end{tabular}


ApÉNDICE A. Estimación de la frecuencia de red mediante un DPLL 158 trifásico y media no lineal

Para señales de entrada monofásicas, un cuadro $\alpha \beta$ ficticio debe ser introducido con el objetivo de emular un sistema de potencia trifásico. El valor de entrada $\left(v_{i n}\right)$ es por lo tanto asignado a $v_{\beta}, \mathrm{y} v_{\alpha}$ e internamente es generado como tensión de cuadratura, como propone Karimi et al. (2004), ver figura A.3b. En este análisis, cinco aproximaciones diferentes se han considerado para estimar $v_{\alpha}$. Estas aproximaciones también son desarrolladas por Choi et al. (2006). Otras contribuciones se pueden encontrar en la literatura. Como ejemplo, un PLL basado en transformación de Hilbert se presenta en Saitou et al. (2003) para sistemas monofásicos para estimar $v_{\alpha}$. Aunque, conduce a un sistema no casual que no es realizable de forma práctica, siendo necesario aproximar la transformación a través del uso de un filtro de respuesta a impulsos finitos (FIR), realizado en Silva et al. (2004). Por esta razón, este método no ha sido considerado en este trabajo.

\section{A.2.1.1. Método de tabla de memoria}

La componente $\alpha$ puede ser estimada como la componente de cuadratura de la tensión a partir de la expresión siguiente:

$$
\begin{gathered}
v_{\alpha}=\hat{V} \cos (\hat{\omega} t)=(-1) \hat{V} \sin \left(\hat{\omega} t-\frac{\pi}{2}\right) . \\
v_{\alpha} \approx(-1) \cdot v_{\text {in }}\left(t-\frac{\hat{T}}{4}\right) .
\end{gathered}
$$

Además, la estimación de $v_{\alpha}$ puede ser obtenida de la señal de entrada $v_{\text {in }}$ con un retraso de $1 / 4$ de periodo multiplicando dicho valor por $(-1)$.

\section{A.2.1.2. Método de amplitud y fase estimadas}

Asumiendo que la señal de entrada es una función seno pura y considerando $v_{\beta}=v_{i n}, v_{\alpha}$ es entonces estimada como $v_{\alpha}=\hat{V} \cdot \cos (\hat{\theta})$, siendo $\hat{V}$ una amplitud estimada y un ángulo de fase estimado $\hat{\theta}$, como se analiza en la sección A.2.2.

\section{A.2.1.3. Método de filtro de primer orden}

Un filtro de primer orden de paso bajo cuya frecuencia de corte $\left(\omega_{c}\right)$ tiene el mismo valor que la frecuencia estimada $(\hat{\omega})$ se ha implementado para obtener la componente $\alpha$ de acuerdo a las expresiones siguientes:

$$
\begin{gathered}
v_{\alpha}=\hat{V} \cos (\hat{\omega} t)=\hat{V} \sin (\hat{\omega} t)-2 \cdot \frac{\hat{V}}{\sqrt{2}} \sin \left(\hat{\omega} t-\frac{\pi}{4}\right) \\
v_{\alpha} \approx v_{i n}-2 \cdot \frac{\hat{V}}{\sqrt{2}} \sin \left(\hat{\omega} t-\frac{\pi}{4}\right)=v_{\text {in }}-2 \cdot L P F_{1}\left(v_{i n}\right),
\end{gathered}
$$


donde $L P F_{1}\left(v_{i n}\right)$ es la salida del filtro de primer orden de paso bajo cuando la entrada es $v_{i n}$.

\section{A.2.1.4. Método de filtro de segundo orden}

De forma similar al método anterior, un filtro de segundo orden de paso bajo es propuesto para obtener la componente $\alpha$. En este caso, la frecuencia natural $\left(\omega_{n}\right)$ del filtro tiene el mismo valor que la frecuencia estimada $(\hat{\omega})$, el ratio de amortiguamiento es $\zeta=1 / \sqrt{2}$ y la diferencia de ángulo de fase introducida es $\pi / 2$. Las siguientes ecuaciones indican lo descrito anteriormente.

$$
\begin{gathered}
v_{\alpha}=\hat{V} \cos (\hat{\omega} t)=-\sqrt{2} \cdot \frac{\hat{V}}{\sqrt{2}} \sin \left(\hat{\omega} t-\frac{\pi}{2}\right), \\
v_{\alpha} \approx-\sqrt{2} \cdot L P F_{2}\left(v_{i n}\right),
\end{gathered}
$$

donde $L P F_{2}\left(v_{i n}\right)$ es la salida del filtro de segundo orden de paso bajo cuando la entrada es $v_{i n}$.

\section{A.2.1.5. Método del filtro pasa-todo}

Un filtro pasa-todo es sugerido también para calcular la componente $\alpha$. En este caso, la frecuencia natural $\omega_{n}$ tiene el mismo valor que la frecuencia estimada multiplicada por $(\sqrt{2}-1)$, siendo el ratio de amortiguamiento $\zeta=1$ y la diferencia entre en ángulo de fase $\pi / 2$.

\section{A.2.2. Estimación del error de fase}

A partir de los componentes $\alpha \beta$, dos propuestas diferentes se han implementado para estimar la señal de salida de la etapa PD, $(\Delta \theta)$. En esos métodos en los cuales se necesita una estimación de la amplitud de la tensión $(\hat{V})$, la cual es obtenida mediante la siguiente expresión.

$$
\hat{V}=\sqrt{v_{\alpha}^{2}+v_{\beta}^{2}}
$$

\section{A.2.2.1. Método usando la función arcotangente}

El ángulo de fase de vector espacial de la tensión se obtiene mediante la función arcotangente de acuerdo a la ecuación A.9.

$$
\theta=\tan ^{-1}\left(\frac{v_{\beta}}{v_{\alpha}}\right)
$$

siendo $\Delta \theta=\theta-\hat{\theta}$, y $\hat{\Delta}$ el ángulo de fase estimado. 
ApÉNDICE A. Estimación de la frecuencia de red mediante un DPLL 160 trifásico y media no lineal

\section{A.2.2.2. Método usando un cuadro síncrono de referencia (SRF)}

El ángulo de fase se obtiene usando un SRF basado en la transformada de Park, como en Karimi-Ghartemani (2006); Ren et al. (2008).

$$
\left[\begin{array}{l}
v_{d} \\
v_{q}
\end{array}\right]=\left[\begin{array}{cc}
\sin \hat{\theta} & \cos \hat{\theta} \\
\cos \hat{\theta} & -\sin \hat{\theta}
\end{array}\right]\left[\begin{array}{l}
v_{\alpha} \\
v_{\beta}
\end{array}\right] .
$$

La diferencia del ángulo de fase es entonces el resultado de dividir $v_{q}$ por $v_{d}$, como en Kaura y Blasko (1997); Choi et al. (2006); Ren et al. (2008), considerando que $v_{\beta}=v_{i n}=V \sin (\omega t)$ y que $v_{\alpha}$ es una estimación de $V \cos (\omega t)$,

$$
\frac{v_{q}}{v_{d}}=\frac{V \sin (\theta-\hat{\theta})}{V \cos (\theta-\hat{\theta})} \approx[\theta-\hat{\theta}]=\Delta \theta
$$

\section{A.3. DPLL trifásico: Solución propuesta}

\section{A.3.1. Estructura del DPLL trifásico}

Asumiendo unas señales de entrada monofásicas, las estructuras PD consideradas en la sección A.2.1 son resumidas en la tabla A.1. Las soluciones propuestas para sistema de potencia trifásicos están basados en estimadores de frecuencia definidos como una media no lineal ponderada de las tres estimaciones monofásicas. Las estructuras DPLL se han aplicado individualmente en cada señal de entrada monofásica, determinando los valores monofásicos correspondientes para la amplitud, el ángulo de fase y la frecuencia respectivamente, ver figura A.4.

Estas soluciones han sido implementadas y validadas bajo perturbaciones de tensión recogidas en un parque eólico español durante varios meses. Adicionalmente, los resultados se han comparado las topologías DPLL trifásicas convencionales basadas tanto en la función arcotangente como en el SRF, utilizadas en Kaura y Blasko (1997); Ren et al. (2008); Chung (2000).

\section{A.3.2. Media no lineal ponderada}

En general, la media aritmética es considerado como un método simple para mostrar la tendencia central de unas muestras. Esta media suele ser usada para mostrar tendencias centrales, pero no constituye un parámetro estadístico robusto y puede verse considerablemente influenciada por efectos externos. En este escenario, la media aritmética puede ser malinterpretada por el valor medio implicando que la mayoría de los valores son mayores o menores de los que son realmente.

Para hacer frente a estos problemas, una media aritmética ponderada suele proponerse, de cara a la combinación de valores medios de las muestras 


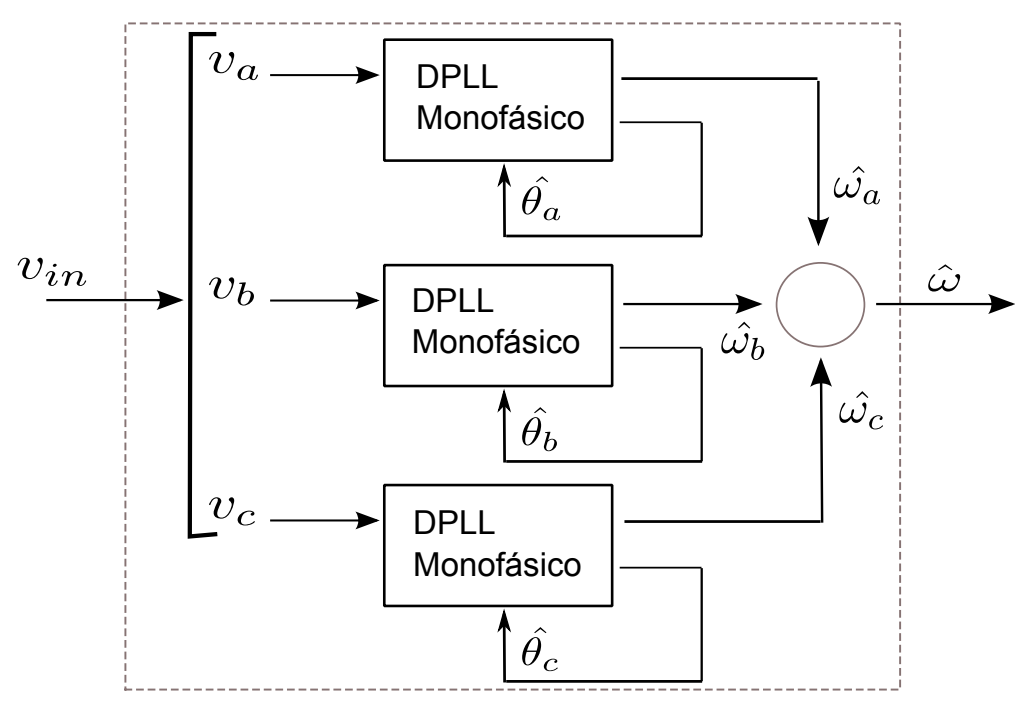

Figura A.4: Solución DPLL trifásico propuesta. Esquema general

con diferentes tamaños. En esos casos, los pesos seleccionados pueden representar unas medidas de la fiabilidad de la influencia entre la media y sus valores respectivos. En nuestro caso, el interés está centrado en el incremento de la influencia de las muestras siendo estas cercanas a la frecuencia en régimen permanente, y por lo tanto decreciendo la influencia de los valores frecuencia afectados por perturbaciones desequilibradas. Bajo este requerimiento, y con el objetivo de estimar el valor de frecuencia de una red robusta, las siguiente media no lineal ponderada es propuesta: dados los datos $f_{1}, f_{2}$ y $f_{3}$ correspondientes a la señal de frecuencia de salida de cada DPLL monofásico, se determina la siguiente expresión.

$$
W A M\left(f_{1}, f_{2}, f_{3}\right)=\sum_{i=1}^{3} \Gamma_{i} f_{i},
$$

donde

$$
\Gamma_{j}=\frac{A_{j}}{\sum_{i=1}^{3} A_{i}},
$$

y $A_{j}=\exp \left(-k\left|f_{r}-f_{j}\right|\right)$ para $j=1,2,3$., siendo $k$ un parámetro constante que incluye la sensibilidad de la frecuencia de red bajo perturbaciones desequilibradas y excursiones del valor de frecuencia nominal de la red $\left(f_{r}\right)$. $f_{r}$ se ha calculado como la frecuencia en régimen permanente siguiente las técnicas clásicas mostradas en Golding (1961); Kumar et al. (1975).

De acuerdo a los parámetros $\Gamma_{j}$, por definición, deben verificar $\Gamma_{j} \geq 0 \mathrm{y}$ $\sum_{i=1}^{3} \Gamma_{i}=1$. 
ApÉNDICE A. Estimación de la frecuencia de red mediante un DPLL 162 trifásico y media no lineal

Finalmente, a continuación, se describen algunas consideraciones relevantes sobre la implementación del proceso para las diferentes topologías.

\section{A.3.3. Inicialización y comparación del ángulo de fase}

En todas las aproximaciones, un bloque de saturación se ha incluido para preservar el valor estimado de fase $(\hat{\theta})$ dentro del intervalo $[0,2 \pi]$. De hecho, y considerando que la función arcotangente tiene un dominio en $\Re$ con un rango limitado entre $(-\pi / 2, \pi / 2)$, un valor nulo de arcotangente puede corresponder tanto a un ángulo de fase $\pi$ como a 0 . Este hecho debe ser tenido en cuenta para realizar la etapa de comparación del ángulo de fase de una manera apropiada. Existen varias soluciones para incluir ambos términos en el mismo rango. Por ejemplo, usando el valor $v_{\alpha}$ para ajustar el valor del arcotangente en un rango $[0,2 \pi)$ y siendo $v_{\beta} \neq 0$.

$$
\tan ^{-1}\left(\frac{v_{\beta}}{v_{\alpha}}\right)= \begin{cases}\left|\tan ^{-1}\left(\frac{v_{\beta}}{v_{\alpha}}\right)\right| \cdot \operatorname{sign}\left(v_{\beta}\right) & \text { if } v_{\alpha}<0 \\ \pi / 2 \cdot \operatorname{sign}\left(v_{\beta}\right) & \text { if } v_{\alpha}=0 \\ \left(\pi-\left|\tan ^{-1}\left(\frac{v_{\beta}}{v_{\alpha}}\right)\right|\right) \cdot \operatorname{sign}\left(v_{\beta}\right) & \text { if } v_{\alpha}>0\end{cases}
$$

Para $v_{\beta}=0$ :

$$
\tan ^{-1}\left(\frac{v_{\beta}}{v_{\alpha}}\right)= \begin{cases}0 & \text { if } v_{\text {alpha }}<0 \\ \text { undefined } & \text { if } v_{\alpha}=0 \\ \pi & \text { if } v_{\alpha}>0\end{cases}
$$

En este caso, los resultados se incluyen en el rango $(-\pi, \pi]$, que puede ser trasladado al rango $[0,2 \pi)$ añadiendo $2 \pi$ a los valores de ángulo negativos. Este procedimiento se conoce como la función atan 2 que es ampliamente utilizada en diversos lenguajes de programación.

\section{A.3.4. Ajuste de PI}

El método del óptimo simétrico (SOM) es usado para determinar los parámetros del PI. Este método es descrito para propósitos generales en Leonhard (2001). En Kaura y Blasko (1997) y da Silva et al. (2006), el uso del método SOM se extiende a los algoritmos PLL.

La función de transferencia en lazo abierto para el esquema de control es:

$$
G_{o l}=\left(K_{p l l} \cdot \frac{1+s T_{p l l}}{s T_{p l l}}\right)\left(\frac{1}{1+s T_{s}}\right)\left(\frac{1}{s}\right),
$$

donde $T_{s}$ es el periodo de muestreo, y $K_{p l l}$ y $T_{p l l}$ son los parámetros del controlador PI respectivamente. De acuerdo con este método, los parámetros PI son calculados asumiendo que la amplitud y la fase de $G_{o l}$ deben ser simétricas con respecto a la frecuencia de cruce $\omega_{c}$-media geométrica de las dos frecuencias límite en el ancho de banda de $G_{o l}$ - 


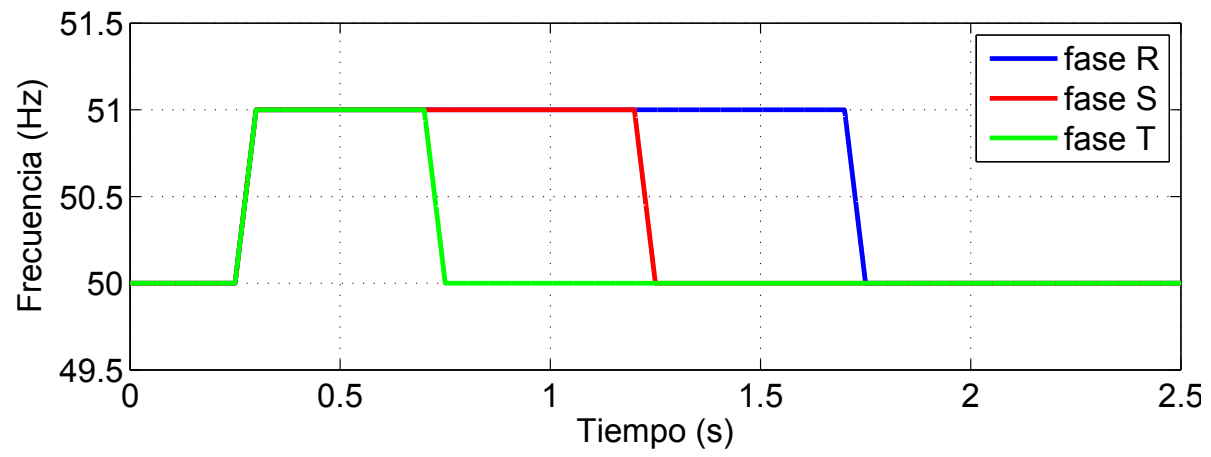

(a) Pasos de la frecuencia simulada.

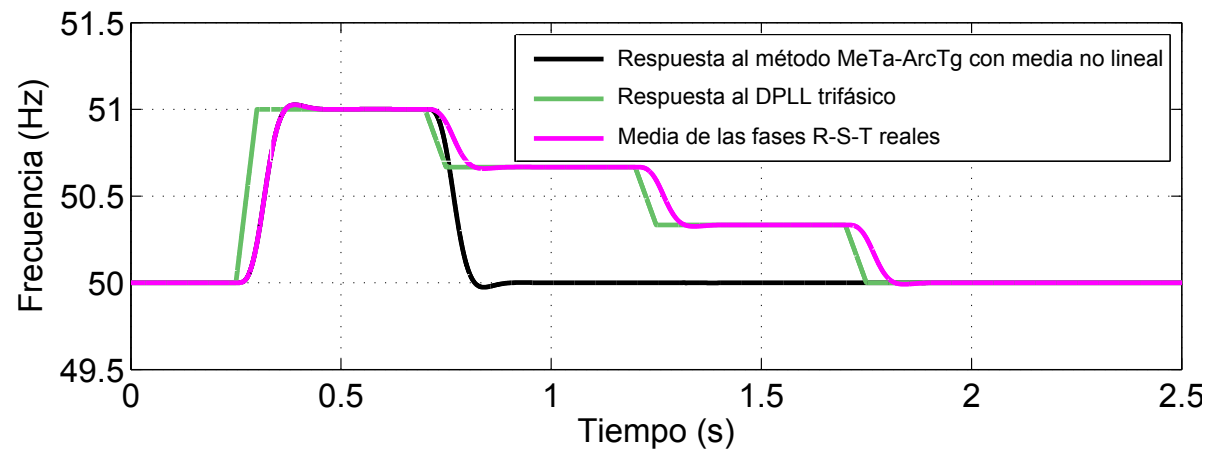

(b) Respuesta de la frecuencia con MeTa-SRF.

Figura A.5: Cambios en la respuesta de la frecuencia con los pasos simulados para el método MeTa-ArcTg. 
ApÉNDICE A. Estimación de la frecuencia de red mediante un DPLL

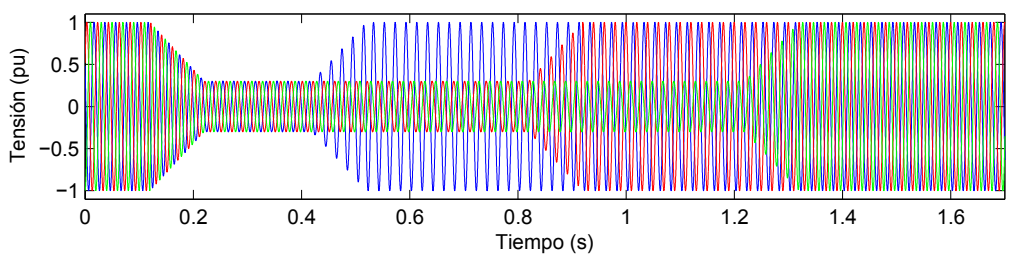

(a) Hueco de tensión simulado.

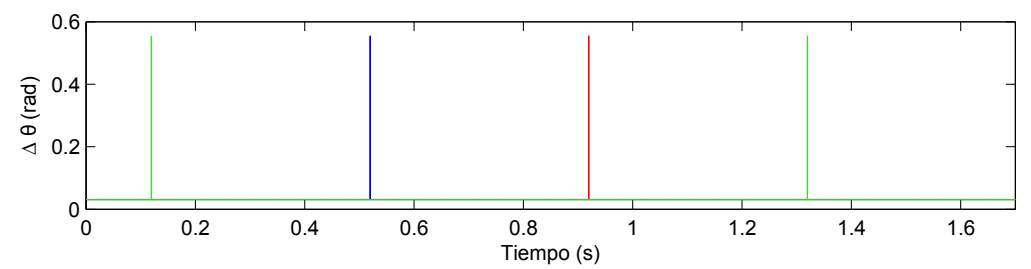

(b) Saltos del ángulo de fase durante el hueco de tensión.

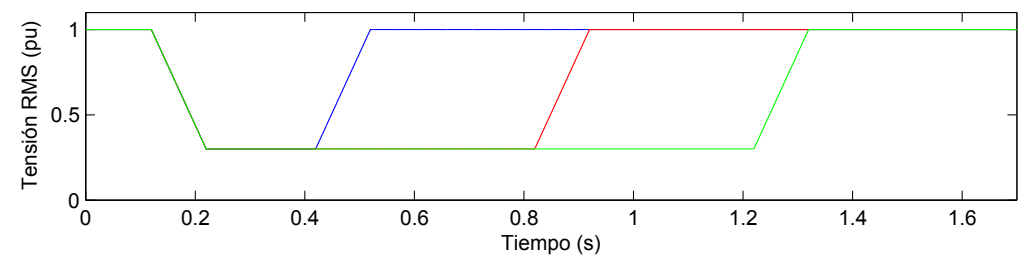

(c) Tensión RMS durante el hueco de tensión.

Figura A.6: Hueco de tensión simulado con saltos de fase y amplitud. 


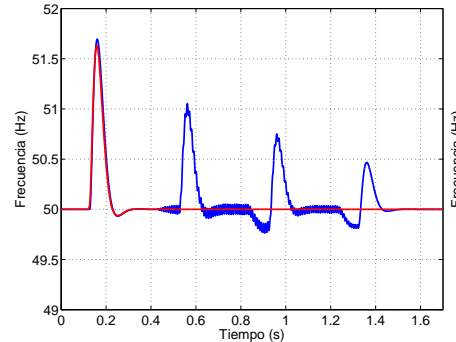

(a) MeTa-ArcTg

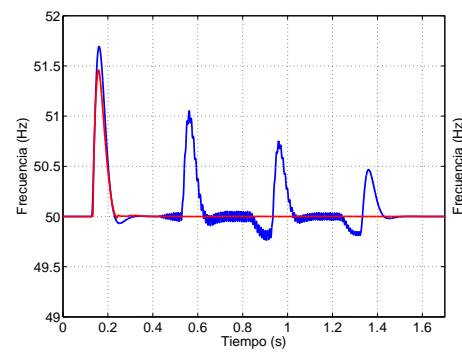

(c) $\mathrm{EPhA}-\mathrm{ArcTg}$

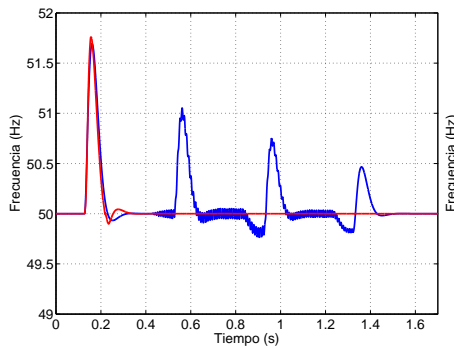

(e) $1 \mathrm{OrF}-\mathrm{ArcTg}$

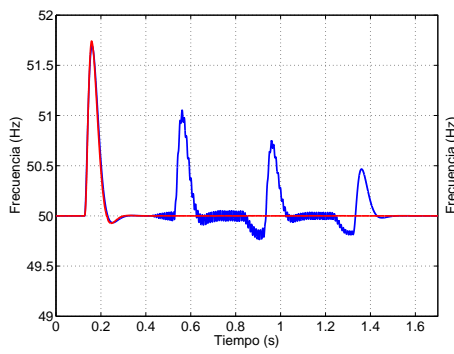

(g) $2 \mathrm{OrF}-\mathrm{ArcTg}$

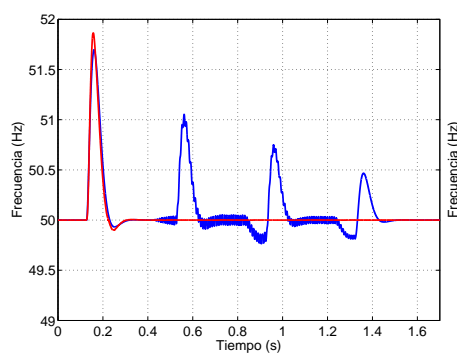

(i) $\mathrm{AllF}-\mathrm{ArcTg}$

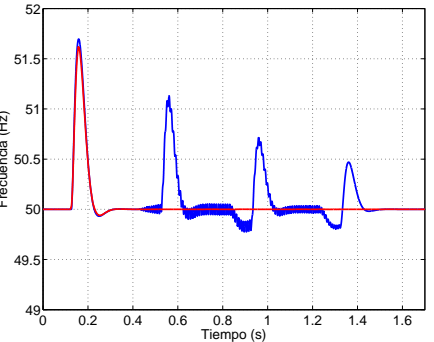

(b) MeTa-SRF

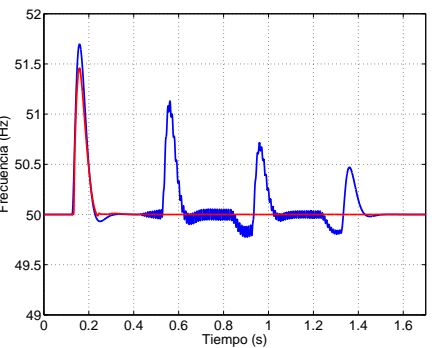

(d) EphA-SRF

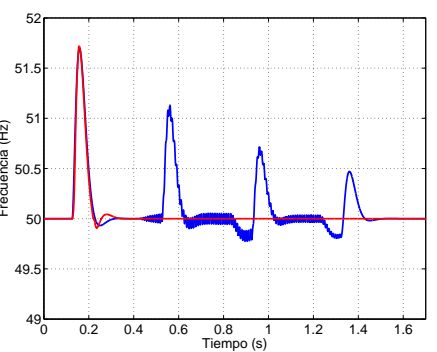

(f) $1 \mathrm{OrF}-\mathrm{SRF}$

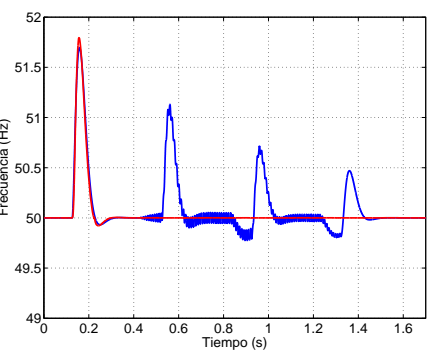

(h) $2 \mathrm{OrF}-\mathrm{SRF}$

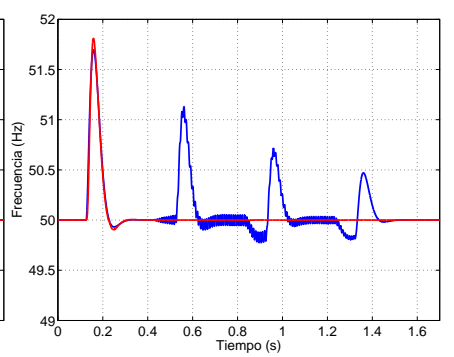

(j) AllF-SRF

Figura A.7: Comparación de las estimaciones de frecuencia para un hueco de tensión simulado: Topología DPLL trifásico (línea azul) y media ponderada no lineal (línea roja) 
ApÉNDICE A. Estimación de la frecuencia de red mediante un DPLL 166 trifásico y media no lineal

\section{A.4. Validación de la metodología no lineal pro- puesta}

La figura A.5 presenta la respuesta de un método MeTa-ArcTg sobre unos cambios simulados de frecuencia en comparación con una topología DPLL trifásica y la media de las tres fases. Adicionalmente, una perturbación de hueco de tensión simulada se usa con el objetivo de validar la media no lineal ponderada propuesta. La figura A.6 muestra la evolución de las tensiones de fase en el tiempo, donde perturbaciones equilibradas y desequilibradas son consideradas. La frecuencia de la señal se fija en $50 \mathrm{~Hz}$. Un salto de ángulo de fase de $\pi / 6$ se incluye al principio de cada recuperación de cada hueco de tensión individual por fase. En total, seis saltos del ángulo de fase han sido implementados, tres al principio de la perturbación equilibrada, ocurriendo simultáneamente en todas las fases, y el resto en la recuperación de cada fase. Un filtro paso bajo se aplica a la salida de los algoritmos DPLL. La figura A.7 muestra los resultados para la frecuencia instantánea estimada para diferentes estructuras de PD (ver tabla A.1), comparándolas con la solución DPLL trifásica. Como se puede apreciar, los saltos simultáneos del hueco de tensión equilibrado producen variaciones de frecuencia instantánea similares en todas las estructuras PD. El cuadro $\alpha \beta$ del método EPhA presenta la menor variación de la frecuencia, incluyendo las topologías de DPLL trifásico. Para el resto de huecos de tensión desequilibrados, donde un salto de ángulo de fase ocurre para cada recuperación de fase, la media no lineal ponderada propuesta genera resultados destacables en comparación con las topologías de DPLL trifásico. De hecho, cada salto de ángulo de fase produce una variación considerable para la solución DPLL trifásico (entre 1 y $0.5 \mathrm{~Hz}$ ), mientras que los resultados con la media no lineal ponderada son prácticamente iguales a la frecuencia original con unas variaciones muy pequeñas (entre 1 y $5 \mathrm{mHz}$ ).

\section{A.5. Resultados experimentales}

Los huecos de tensión reales recogidos a lo largo de varios meses en un parque eólico español se han utilizado para evaluar la topología de media no lineal ponderada propuesta. Estas mediciones se han llevado a cabo utilizando un registrador de calidad eléctrica Fluke 1760. Para más información se puede consultar Gomez-Lazaro et al. (2007). Todos los tipos de PD indicadas en la tabla A.1 han sido testeadas bajo diferentes condiciones de perturbaciones equilibradas y desequilibradas comparando la evolución de la estimación de la frecuencia de red instantánea con lo obtenido con el método convencional DPLL trifásico. Un filtro paso bajo ha sido aplicado a la salida de los algoritmos DPLL. 


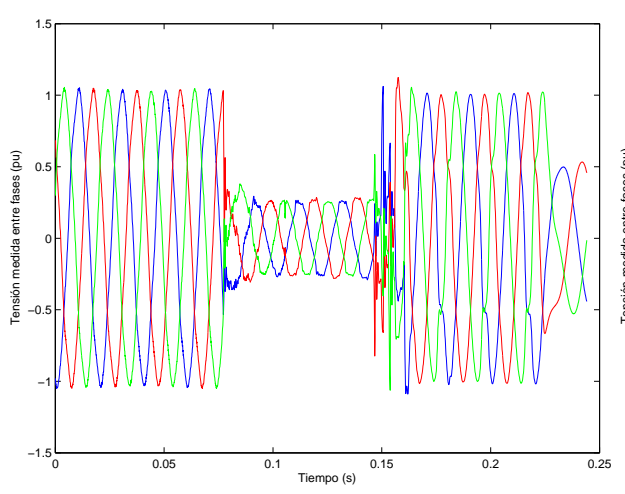

(a) Hueco de tensión trifásico

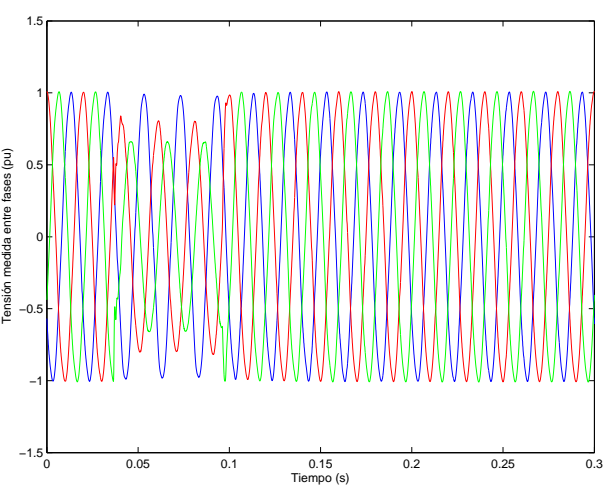

(b) Hueco de tensión bifásico

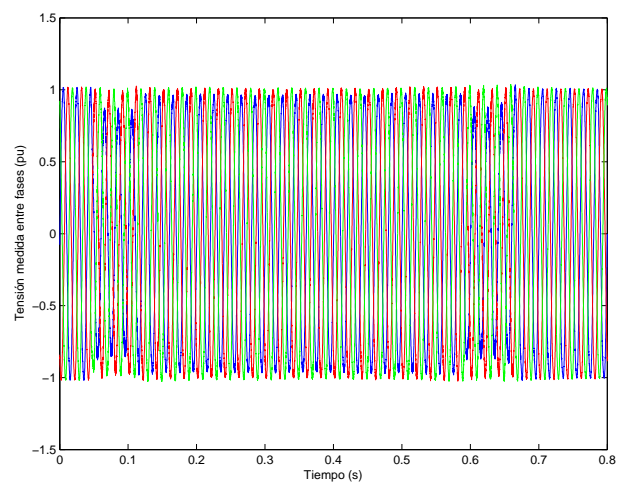

(c) Hueco de tensión monofásico

Figura A.8: Ejemplos reales de perturbaciones (equilibradas y desequilibradas) 
APÉNDICE A. Estimación de la frecuencia de red mediante un DPLL trifásico y media no lineal

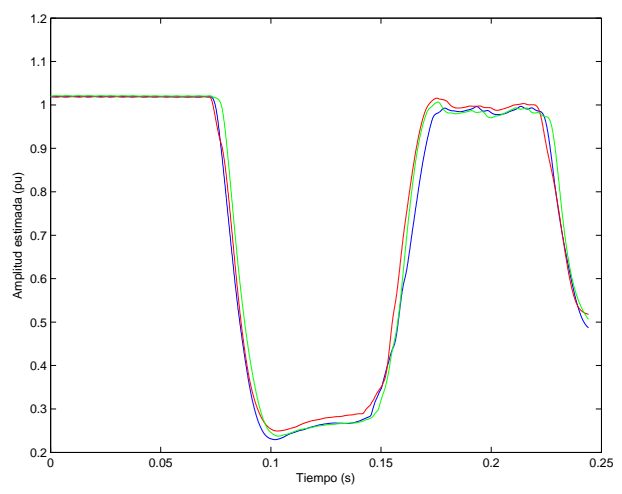

(a) Hueco de tensión trifásico

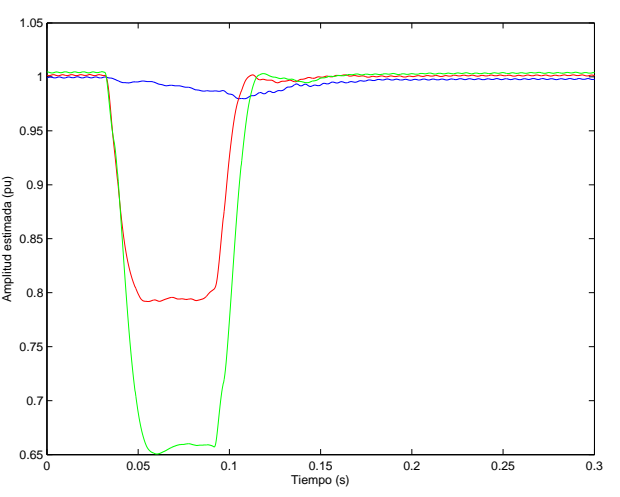

(b) Hueco de tensión bifásico

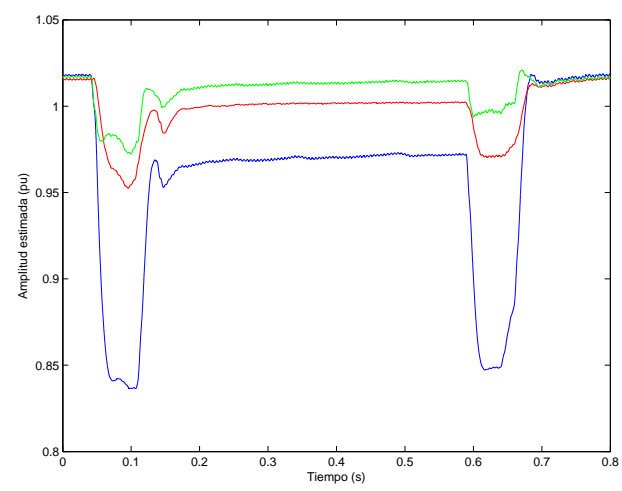

(c) Hueco de tensión monofásico

Figura A.9: Ejemplos reales de perturbaciones (equilibradas y desequilibradas). Amplitud estimada 


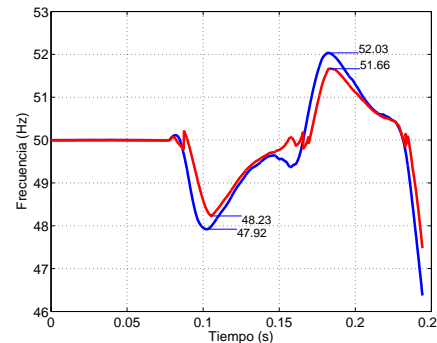

(a) MeTa-ArcTg

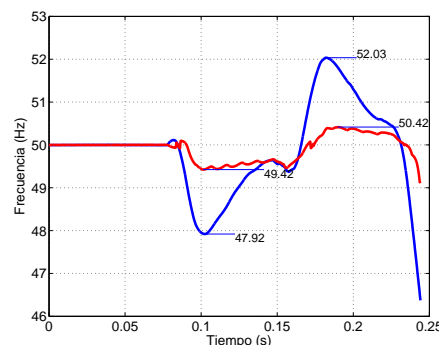

(c) $\mathrm{EPhA}-\mathrm{ArcTg}$

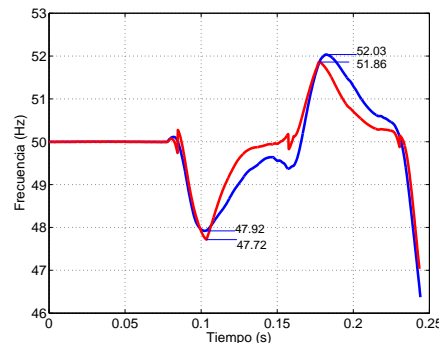

(e) $1 \mathrm{OrF}-\mathrm{ArcTg}$

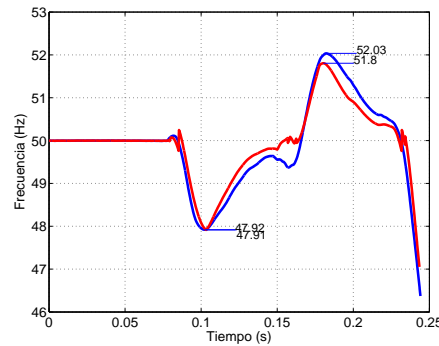

(g) $2 \mathrm{OrF}-\mathrm{ArcTg}$

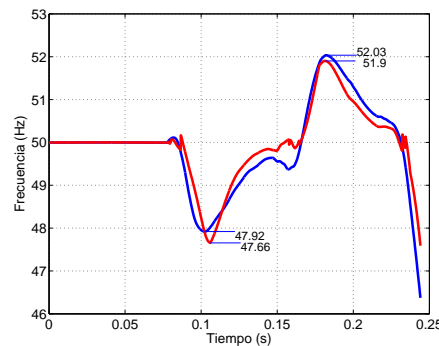

(i) AllF-ArcTg

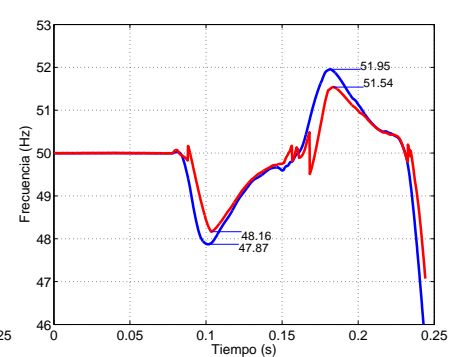

(b) MeTa-SRF

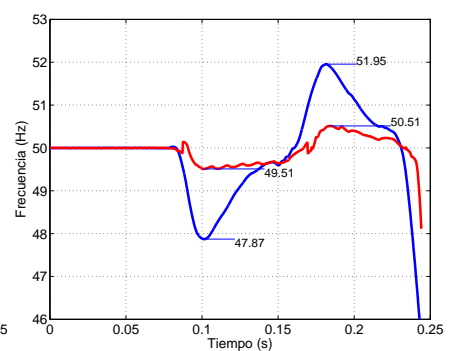

(d) EphA-SRF

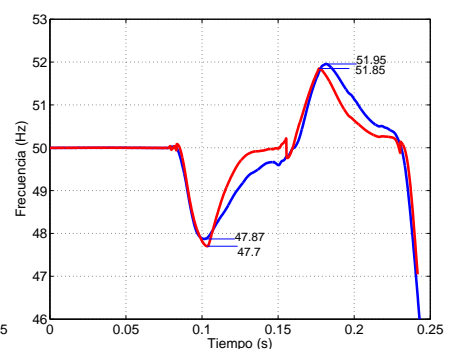

(f) $1 \mathrm{OrF}-\mathrm{SRF}$

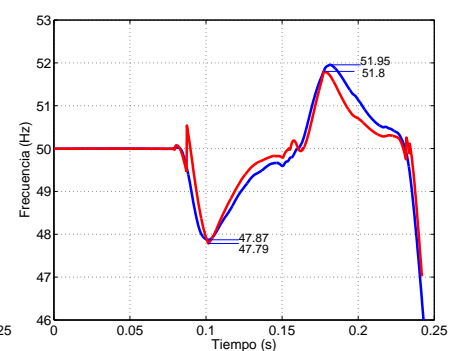

(h) $2 \mathrm{OrF}-\mathrm{SRF}$

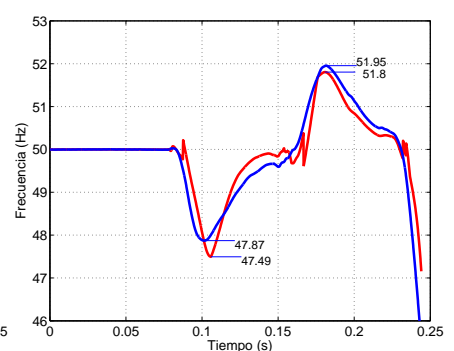

(j) AllF-SRF

Figura A.10: Comparación de la estimación de frecuencia para el hueco de tensión trifásico real: Topología DPLL trifásico (línea azul) y media ponderada no lineal (línea roja) 
APÉNDICE A. Estimación de la frecuencia de red mediante un DPLL 170 trifásico y media no lineal

\section{A.5.1. Estimación de frecuencia en hueco de tensión trifásico}

La figura A.9a muestra un hueco de tensión trifásico real registrado el 26 de abril de 2007, en Parque Eólico Moralejo I -España. La caída de tensión durante el hueco es de $0.75 \mathrm{pu}$, siendo su duración de casi $100 \mathrm{~ms}$. Después del intervalo de recuperación, se produce otra reducción de tensión de hasta $0.5 \mathrm{pu}$. La figura A.10 muestra los valores estimados durante el hueco de tensión trifásico real con las diferentes topologías de PD presentadas en la sección A.3. En estos resultados, dos aproximaciones diferentes han sido consideradas para estimar la frecuencia de red: la topología DPLL trifásico convencional (línea azul) y la media ponderada no lineal descrita en la sección A.3.2 (línea roja). En referencia al DPLL trifásico convencional, la etapa PD se ha implementado considerando la ecuación A.1 y la segunda etapa consiste en un método SRF. De forma similar a los resultados del hueco de tensión simulado, todas las estructuras de PD propuestas muestran una desviación de frecuencia durante el hueco de tensión. De hecho, el método del cuadro $\alpha \beta$ EPhA reduce significativamente las variaciones de frecuencia instantánea en comparación con la topología DPLL trifásico.

\section{A.5.2. Estimación de frecuencia en hueco de tensión bifásico}

La figura A.9b muestra un hueco de tensión bifásico real registrado el 2 de febrero de 2009, en Parque Eólico Moralejo I -España. La caída de tensión durante el hueco es de $0.35 \mathrm{pu}$, siendo su duración de casi $100 \mathrm{~ms}$. De forma similar al apartado A.5.1, la figura A.11 muestra los valores estimados durante el hueco de tensión bifásico real con las diferentes topologías de PD presentadas. Como se puede apreciar, la media no lineal propuesta da, en todos los casos, menores desviaciones de frecuencia durante la perturbación desequilibrada. El método con el cuadro $\alpha \beta$ EPhA presenta las menores desviaciones.

\section{A.5.3. Estimación de frecuencia en hueco de tensión monofási- co}

La figura A.9c muestra dos huecos de tensión monofásico consecutivos registrados el 5 de noviembre de 2008, en Parque Eólico Moralejo I-España. Las caídas de tensión durante los huecos son de 0.15 pu en ambos, siendo sus duraciones sobre los $75 \mathrm{~ms}$ cada uno.

La figura A.12 muestra los valores estimados durante el hueco de tensión monofásico real con las diferentes topologías de PD presentadas. Como en el caso anterior, la media no lineal propuesta da, en todos los casos, menores desviaciones de frecuencia durante la perturbación desequilibrada. Finalmente, el método Método de amplitud y fase estimadas (EPhA) entrega las menores desviaciones bajo la presencia de esas perturbaciones desequili- 


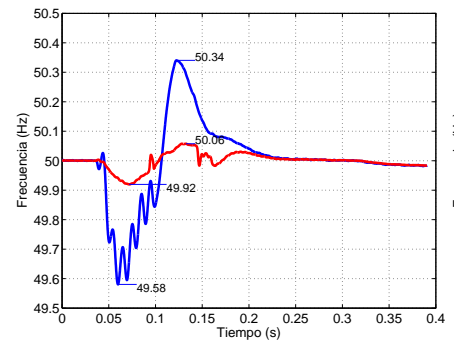

(a) MeTa-ArcTg

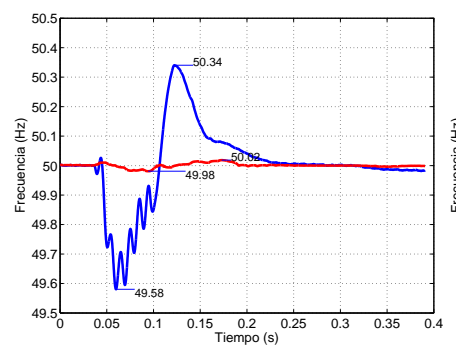

(c) $\mathrm{EPhA}-\mathrm{ArcTg}$

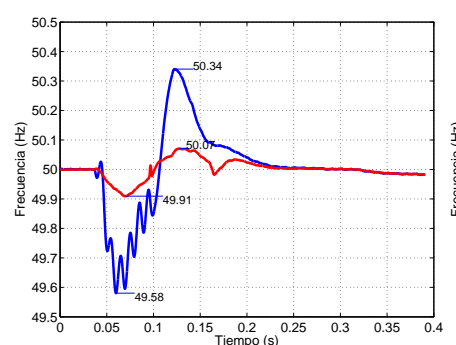

(e) $1 \mathrm{OrF}-\mathrm{ArcTg}$

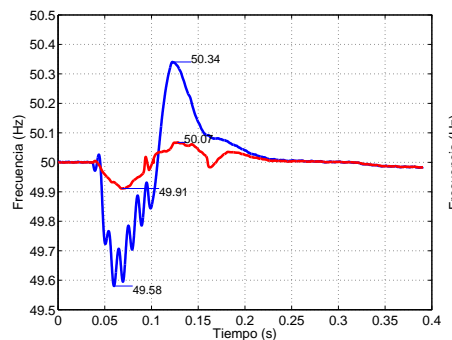

(g) $2 \mathrm{OrF}-\mathrm{ArcTg}$

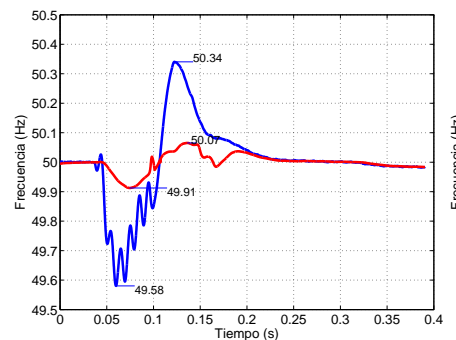

(i) AllF-ArcTg

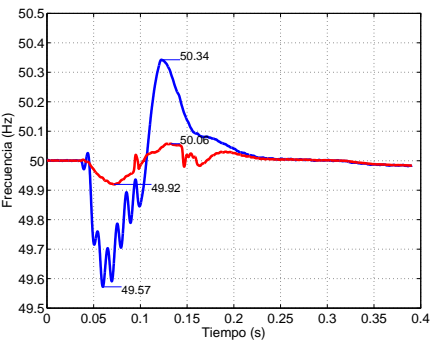

(b) MeTa-SRF

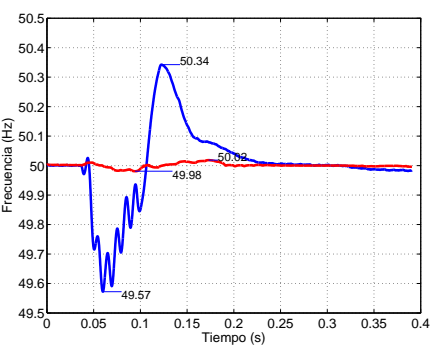

(d) EphA-SRF

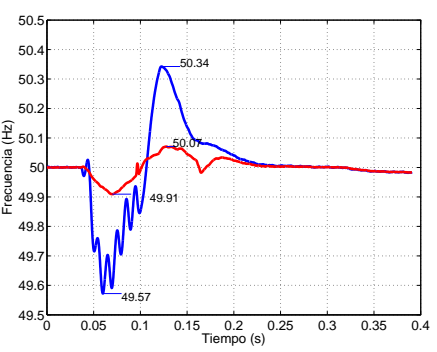

(f) $1 \mathrm{OrF}-\mathrm{SRF}$

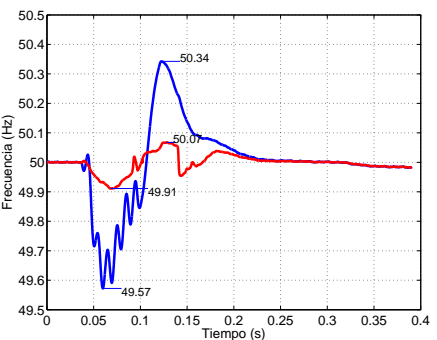

(h) 2OrF-SRF

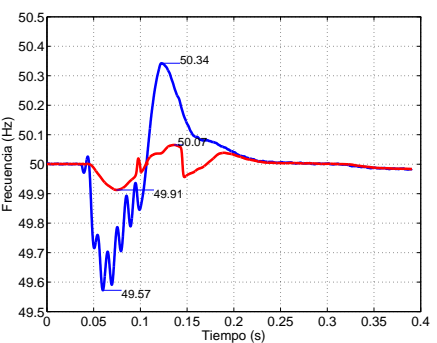

(j) AllF-SRF

Figura A.11: Comparación de la estimación de frecuencia para el hueco de tensión bifásico real: Topología DPLL trifásico (línea azul) y media ponderada no lineal (línea roja) 
ApÉNDICE A. Estimación de la frecuencia de red mediante un DPLL 172 trifásico y media no lineal

bradas.

\section{A.6. Conclusiones}

En este apéndice se han detallado las contribuciones anteriores en la estimación de frecuencia y se han descrito las mayoría de métodos DPLL incluidos en la literatura en la sección A.2. A partir de estas contribuciones, unas nuevas estructuras de PD basadas en valores monofásicos de tensión son propuestas y validadas para estimar la frecuencia instantánea de red en los sistemas eléctricos de potencia bajo la presencia de perturbaciones. La frecuencia estimada del sistema trifásico se obtiene como una media no lineal ponderada de las componentes monofásicas de salida.

Tres huecos de tensión reales recogidos en un parque eólico español a lo largo de varios meses han sido usados para validar la soluciones mediante la media no lineal ponderada propuestas. En todos los casos, los resultados obtenidos proporcionan una estimación con menor desviación de frecuencia bajo la presencia de perturbaciones en comparación con los métodos DPLL trifásicos convencionales previos. De hecho, el método Método de amplitud y fase estimadas (EPhA) ofrece los resultados más remarcables, minimizando las desviaciones de frecuencia durante las perturbaciones equilibradas y desequilibradas.

Este método de estimación de frecuencia de red puede ser utilizado en el control de potencia activa para reducir la variabilidad a corto plazo. Al usarlo en combinación con los métodos desarrollados en se pueden establecer los parámetros de $R_{\max }$ y Delta que se deben usar para mantener el sistema en condiciones óptimas de funcionamiento a partir de la energía primaria cedida por las instalaciones eólicas. 


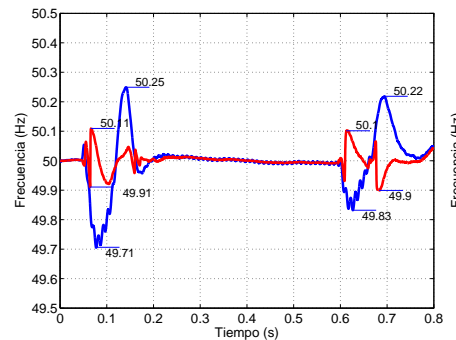

(a) $\mathrm{MeTa}-\mathrm{ArcTg}$

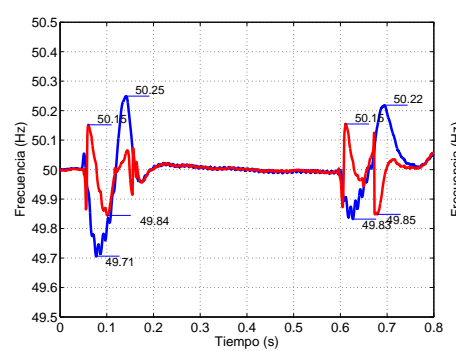

(c) $\mathrm{EPhA}-\mathrm{ArcTg}$

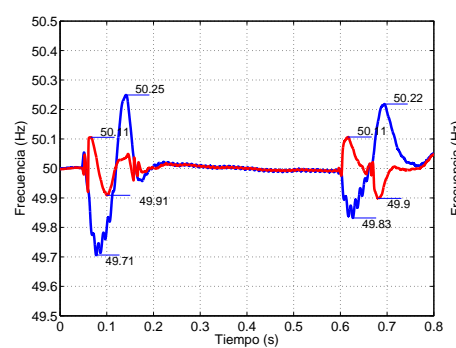

(e) $1 \mathrm{OrF}-\mathrm{ArcTg}$

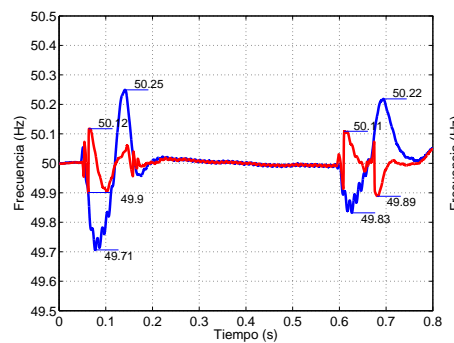

(g) $2 \mathrm{OrF}-\mathrm{ArcTg}$

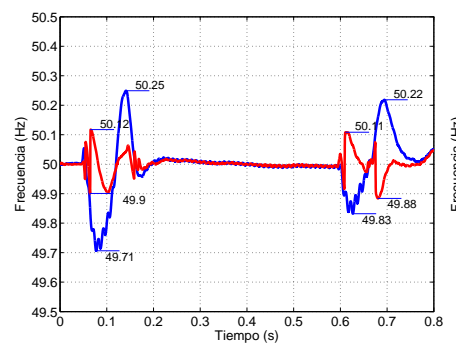

(i) AllF-ArcTg

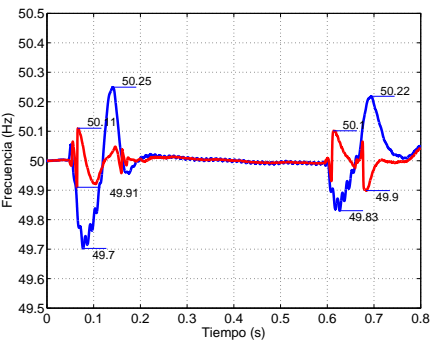

(b) MeTa-SRF

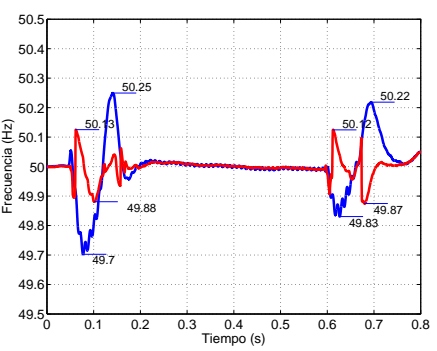

(d) EphA-SRF

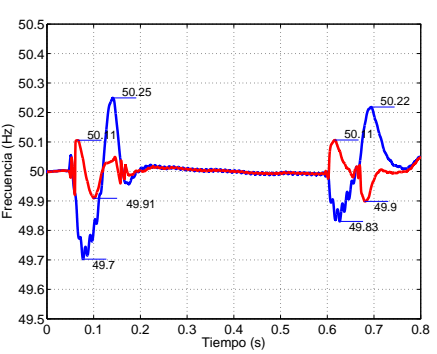

(f) $1 \mathrm{OrF}-\mathrm{SRF}$

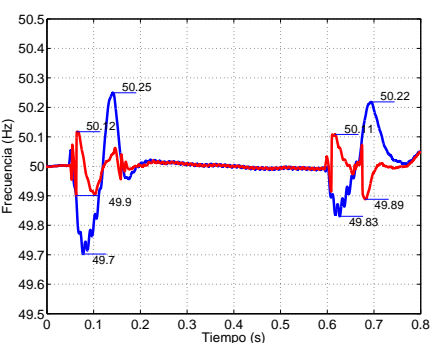

(h) $2 \mathrm{OrF}-\mathrm{SRF}$

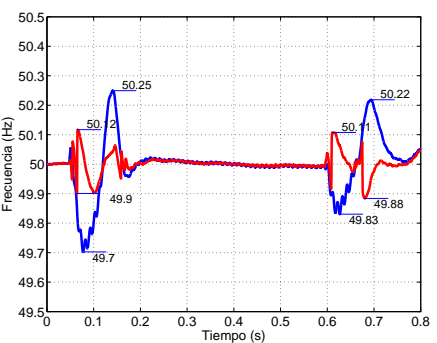

(j) AllF-SRF

Figura A.12: Comparación de la estimación de frecuencia para el hueco de tensión monofásico real: Topología DPLL trifásico (línea azul) y media ponderada no lineal (línea roja) 



\section{Bibliografía}

Abdel-Aal, R., Elhadidy, M. y ShaAhid, S. Modeling and forecasting the mean hourly wind speed time series using gmdh-based abductive networks. Renewable Energy, vol. 34(7), páginas 1686-1699, 2009.

Ackermann, T. Wind power in power systems, vol. 1. Wiley and Sons, 2003.

AEE. Asociación empresarial eólica. www.aeeolica.es.

AKke, M. Frequency estimation by demodulation of two complex signals. IEEE Trans. on Power Delivery, vol. 12(1), páginas 157-163, 1997.

Alexiadis, M., Dokopoulos, P. y Sahsamanoglou, H. Wind speed and power forecasting based on spatial correlation models. Energy Conversion, IEEE Transactions on, vol. 14(3), páginas 836-842, 1999.

Alexiadis, M., Dokopoulos, P., Sahsamanoglou, H. y MANOUSARIDIS, I. Short-term forecasting of wind speed and related electrical power. Solar Energy, vol. 63(1), páginas 61-68, 1998.

Alpay, S., Bilir, L., Ozdemir, S. y Ozerdem, B. Wind speed time series characterization by hilbert transform. International journal of energy research, vol. 30(5), páginas 359-364, 2006.

Asari, M., Nanahara, T., Maejima, T., Yamaguchi, K. y Sato, T. A study on smoothing effect on output fluctuation of distributed wind power generation. En Transmission and Distribution Conference and Exhibition 2002: Asia Pacific. IEEE/PES, vol. 2, páginas 938-943. IEEE, 2002.

Banakar, H., LuO, C. y OoI, B. Impacts of wind power minute-to-minute variations on power system operation. Power Systems, IEEE Transactions on, vol. 23(1), páginas 150-160, 2008.

Bendat, J. y Piersol, A. Random data: analysis and measurement procedures, vol. 729. Wiley, 2011. 
Beyer, H., Luther, J., Pahlke, W. y Steinberger-Willms, R. Characteristics of the power output of wind turbines and large scale dispersed wind energy systems. En Proceeding of ECWEC, páginas 606-610. 1990a.

Beyer, H., Luther, J. y Steinberger-Willms, R. Fluctuations in the combined power output from geographically distributed grid coupled wecsan analysis in the frequency domain. Wind Engineering, vol. 14, páginas 179-192, 1990b.

Bossanyi, E. Short-term wind prediction using kalman filters. Wind Engineering, vol. 9(1), páginas 1-8, 1985.

Bouffard, F. y Galiana, F. Stochastic security for operations planning with significant wind power generation. En Power and Energy Society General Meeting-Conversion and Delivery of Electrical Energy in the 21st Century, 2008 IEEE, páginas 1-11. IEEE, 2008.

Brand, A., Gibescu, M. y Boer, W. D. Variability and predictability of large-scale wind energy in the netherlands. En Wind Power. Croatia: InTech, 2010.

Brett, A. y Tuller, S. The autocorrelation of hourly wind speed observations. Journal of Applied Meteorology, vol. 30, páginas 823-833, 1991.

Burton, T., Sharpe, D., Jenkins, N. y Bossanyi, E. Wind energy: handbook. Wiley Online Library, 2001.

Cadenas, E. y Rivera, W. Short term wind speed forecasting in la venta, oaxaca, méxico, using artificial neural networks. Renewable Energy, vol. 34(1), páginas 274-278, 2009.

Carraretto, C. Power plant operation and management in a deregulated market. Energy, vol. 31(6), páginas 1000-1016, 2006.

Cataliotti, A., Cosentino, V. y Nuccio, S. A phase-locked loop for the synchronization of power quality instruments in the presence of stationary and transient disturbances. IEEE Trans. on Instrumentation and Measurement,, vol. 56(6), páginas 2232-2239, 2007.

Chor, J.-W., Kim, Y.-K. y Kim, H.-G. Digital pll control for single-phase photovoltaic system. IEE Proceedings Electric Power Applications, vol. 153(1), páginas 40-46, 2006.

Chung, S.-K. Phase-locked loop for grid-connected three-phase power conversion systems. IEE Proceedings -Electric Power Applications, vol. 147(3), páginas 213-219, 2000.

CNE. Información estadística sobre las ventas de energía del régimen especial. Informe técnico, Comisión Nacional de la Energía, 2013. 
Colorado. Wind integration study for public service of colorado. Informe técnico, EnerNex Corp., 2006.

Committee, I. E. ET AL. Iec 61400-1: Wind turbines part 1: Design requirements. Informe técnico, International Electrotechnical Commission, 2005.

Contaxis, G. y Kabouris, J. Short term scheduling in a wind/diesel autonomous energy system. Power Systems, IEEE Transactions on, vol. 6(3), páginas 1161-1167, 1991.

Cooley, J., Lewis, P. y Welch, P. The application of the fast fourier transform algorithm to the estimation of spectra and cross-spectra. Journal of Sound and Vibration, vol. 12(3), páginas 339-352, 1970.

Costa, A., Crespo, A. y Migoya, E. First results from a prediction project. En Proceedings of the European Wind Energy Conference EWEC03. 2003.

Courtney, M. y Troen, I. Wind speed spectrum from one year of continuous $8 \mathrm{hz}$ measurements. En 9th Symposium on Turbulence and Diffusion, vol. 30, página 1990. 1990.

Cutululis, N. A., Zeni, L. y Sørensen, P. Reliability indexes for offshore wind power production under extreme wind conditions. En European Wind Energy Conference and Exhibition (EWEC2010). 2010.

Damousis, I., Alexiadis, M., Theocharis, J. y Dokopoulos, P. A fuzzy model for wind speed prediction and power generation in wind parks using spatial correlation. Energy Conversion, IEEE Transactions on, vol. 19(2), páginas 352-361, 2004.

Damousis, I. y Dokopoulos, P. A fuzzy expert system for the forecasting of wind speed and power generation in wind farms. En Power Industry Computer Applications, 2001. PICA 2001. Innovative Computing for Power-Electric Energy Meets the Market. 22nd IEEE Power Engineering Society International Conference on, páginas 63-69. IEEE, 2001.

DANY, G. Power reserve in interconnected systems with high wind power production. En Power Tech Proceedings, 2001 IEEE Porto, vol. 4, páginas 6-pp. IEEE, 2001.

Dash, P. K., Swain, D. P., Routray, A. y Liew, A. C. An adaptive neural network approach for the estimation of power system frequency. Electric Power Systems Research, vol. 41, páginas 203-210, 1997.

DAVEnPorT, A. The spectrum of horizontal gustiness near the ground in high winds. Quarterly Journal of the Royal Meteorological Society, vol. 87(372), páginas 194-211, 1961. 
De Vos, K., Petoussis, A. G., Driesen, J. y Belmans, R. Revision of reserve requirements following wind power integration in island power systems. Renewable Energy, vol. 50, páginas 268-279, 2013.

DJuRIC, M. y DJuRISIC, Z. Frequency measurement of distorted signals using fourier and zero crossing techniques. Electric Power Systems Research, vol. 78(8), páginas 1407-1415, 2008.

Duric, M., Terzija, V. y Skokluev, I. Power system frequency estimation utilizing the newton-raphson method. Electrical Engineering, vol. 77, páginas 221-226, 1994.

EIRGRID. Eirgrid grid code. Informe técnico, Eirgrid, 2009. Version 3.3.

El-Fouly, T., El-Saadany, E. y Salama, M. Grey predictor for wind energy conversion systems output power prediction. Power Systems, IEEE Transactions on, vol. 21(3), páginas 1450-1452, 2006.

ELtRA. Winds turbines connected to grid with voltages below $100 \mathrm{kV}$. Informe técnico, Eltra and Elkraft system, 2004.

Enercon. Enercon e-70. Informe técnico, Technical description, 2011.

EnERginet. Technical regulations for thermal power stations units of 1.5 mw and higher. Informe técnico, Energinet, 2007.

Ensslin, C., Hoppe-Kilpper, M. y Rohrig, K. Wind power integration in power plant scheduling schemes. En Proceedings of EWEC Special Topic conference, Kassel, páginas 25-27. 2000.

FÉGEANT, O. Masking of wind turbine noise: Influence of wind turbulence on ambient noise fluctuations. Royal Istitute of Technology http://www. vindenergi. foi. se/VindForsk/Rapporter_Vindforsk/Fegeant. pdf, 2002.

Filho, R. S., Seixas, P., Cortizo, P. y Souza, A. Comparison of three single-phase pll algorithms for ups applications. IEEE Trans. on Industrial Electronics,, vol. 55(8), páginas 2923-2932, 2008.

Fink, S., Mudd, C., Porter, K. y Morgenstern, B. Wind energy curtailment case studies. NREL subcontract report, NREL/SR-550-46716, 2009 .

Focken, U. y LAnge, M. Physical approach to short-term wind power prediction. Springer, 2006.

Focken, U., Lange, M., Mönnich, K., Waldl, H., Beyer, H. y Luig, A. Short-term prediction of the aggregated power output of wind farms, a statistical analysis of the reduction of the prediction error by spatial 
smoothing effects. Journal of Wind Engineering and Industrial Aerodynamics, vol. 90(3), páginas 231-246, 2002.

Focken, U., Lange, M. y Walde, H. Previento-a wind power prediction system with an innovative upscaling algorithm. En Proceedings of the European Wind Energy Conference, Copenhagen, Denmark, vol. 276. Citeseer, 2001a.

Focken, U., LAnge, M. y WaldL, H. Reduction of wind power prediction error by spatial smoothing effects. En Proceedings of the European Wind Energy Conference, Copenhagen, Denmark, páginas 822-825. 2001b.

Frandsen, S., Ejsing Jørgensen, H. y Sørensen, J. Relevant criteria for testing the quality of turbulence models. En European Wind Energy Conference and Exhibition. 2007.

Freijedo, F., Doval-Gandoy, J., Lopez, O. y Acha, E. Tuning of phase-locked loops for power converters under distorted utility conditions. IEEE Trans. on Industry Applications,, vol. 45(6), páginas 2039-2047, 2009.

Frías, L., Gastón, M. y Martí, I. A new model for wind energy forecasting focused in the intra-daily markets. En Poster Session of the European Wind Energy Conference EWEC, vol. 7. 2007.

Frost, W., Long, B. y Turner, R. Engineering handbook on the atmospheric environmental guidelines for use in wind turbine generator development. Informe técnico, Tennessee Univ., Tullahoma (USA). Space Inst.; National Aeronautics and Space Administration, Huntsville, AL (USA). George C. Marshall Space Flight Center, 1978.

Gastón, M., Frías, L. y Martí, I. Exploring the limits of wind farm grouping for prediction error compensation. En Proceedings of the European Wind Energy Conference EWEC06. 2006.

Guerstad, J., Aasen, S., Andersson, H., Brevik, I. y Lovseth, J. An analysis of low-frequency maritime atmospheric turbulence. Journal of the atmospheric sciences, vol. 52(15), páginas 2663-2669, 1995.

Golding, J. Counter methods of frequency measurement. British Communication and Electronics Journal, vol. 8, páginas 848-853, 1961.

Gomez-Lazaro, E., Canas, M., Fuentes, J. y Molina-Garcia, A. Characterization of measured voltage dips in wind farms in the light of the new grid codes. En Proc. IEEE Power Tech. Lausanne, 2007.

Gonzalez, G., Diaz-Guerra, B., Soto, F., Lopez, S., Sanchez, I., Usaola, J., Alonso, M. y Lobo, M. Sipreólico-wind power prediction 
tool for the spanish peninsular power system. Proceedings of the CIGR É 40th General Session 86 Exhibition. Parts (France), 2004.

González, G., Lopez, A., Prieto, E., SÁnchez, I. y Lobo, M. Sipreolico: Wind power prediction tool. improvements in the period 2005-2007. En Cigré Session. 2008.

Gøransson, L. y Johnsson, F. Large scale integration of wind power: moderating thermal power plant cycling. Wind Energy, 2010.

Greaves, B., Collins, J., Parkes, J. y Tindal, A. Temporal forecast uncertainty for ramp events. Wind Engineering, vol. 33(4), páginas 309319, 2009 .

HARRIS, R. On the spectrum and auto-correlation function of gustiness in high winds. Electrical Research Association, 1968.

Heggem, T., Lende, R. y Løvseth, J. Analysis of long time series of coastal wind. Journal of the atmospheric sciences, vol. 55(18), páginas 2907-2917, 1998.

Holttinen, H. The impact of large scale wind power production on the nordic electricity system. Informe técnico, VTT Publications, Espoo, 2004.

Holttinen, H. Hourly Wind Power Variations in the Nordic Countries. Wind Energy, vol. 8, páginas 173-195, 2005a.

Holttinen, H. Impact of Hourly Wind Power Variations on the System Operation in the Nordic Countries. Wind Energy, vol. 8, páginas 197-218, 2005b.

Holttinen, H., Meibom, P., Orthi, A., O’Malley, M., Tande, J. O., Estanqueiro, A., Gómez, E., Søder, L., Strbac, G., Smith, J. C. y VAN Hulle, F. Impacts of large amounts of wind power on design and operation of power systems, results of IEA collaboration. Wind Energy, 2010.

Holttinen, H., Milligan, M., Ela, E., Menemenlis, N., Dobschinski, J., Rawn, B., Bessa, R. J., Flynn, D., Gomez-Lazaro, E. y Detlefsen, N. K. Methodologies to determine operating reserves due to increased wind power. Sustainable Energy, IEEE Transactions on, vol. 3(4), páginas 713-723, 2012.

Holttinen, H., Milligan, M., Kirby, B., Acker, T., Neimane, V. y Molinski, T. Using standard deviation as a measure of increased operational reserve requirement for wind power. Wind Engineering, vol. 32(4), páginas 355-377, 2008. 
VAN DER Hoven, I. Power spectrum of horizontal wind speed in the frequency range from 0.0007 to 900 cycles per hour. Journal of Atmospheric Sciences, vol. 14, páginas 160-164, 1957.

Huang, C.-H., Shin, K.-J. y Wang, Y.-J. Frequency estimation of distorted power system signals using a robust algorithm. IEEE Trans. on Power Delivery, vol. 23(1), páginas 41-51, 2008.

HuAng, Z. y Chalabi, Z. Use of time-series analysis to model and forecast wind speed. Journal of wind engineering and industrial aerodynamics, vol. 56(2), páginas 311-322, 1995.

Hurley, T. y Watson, R. An assessment of the expected variability and load following capability of a large penetration of wind power in ireland. En Proceedings of Global Wind Power Conference GWPC, vol. 2. 2002.

IRLANDA. Impact of high levels of wind penetration in 2020 on the single electricity market (sem) . ireland. Informe técnico, Commission for Energy Regulation and Utility Regulator, 2009.

IWEA. Irish Wind Energy Association. www.iwea.com.

Jovcic, D. Phase locked loop system for facts. IEEE Trans. on Power Systems, vol. 18(3), páginas 1116-1124, 2003.

Kaimal, J., Wyngaard, J., Izumi, Y. y Cote, O. Spectral characteristics of surface-layer turbulence. Quarterly Journal of the Royal Meteorological Society, vol. 98(417), páginas 563-589, 1972.

Kamal, L. y JAFRI, Y. Time series models to simulate and forecast hourly averaged wind speed in quetta, pakistan. Solar Energy, vol. 61(1), páginas 23-32, 1997.

Kamwa, I. y SRInivasan, K. A kalman filter-based technique for combined digital estimation of voltage flicker and phase in power distribution systems. European Transactions on Electrical Power, vol. 3(2), páginas 131-142, 1993.

Karimi, H., Karimi-Ghartemani, M. y Iravani, M. Estimation of frequency and its rate of change for applications in power systems. IEEE Trans. on Power Delivery,, vol. 19(2), páginas 472-480, 2004.

Karimi-Ghartemani, M. A novel three-phase magnitude-phase-locked loop system. IEEE Trans. on Circuits and Systems, vol. 53(8), páginas 1792-1802, 2006.

Karimi-Ghartemani, M. y Iravani, M. Wide-range, fast and robust estimation of power system frequency. Electric Power Systems Research, vol. 65(2), páginas 109-117, 2003. 
Kariniotakis, G., Nogaret, E., Dutton, G., Halliday, J., AndroutSOS, A. ET AL. Evaluation of advanced wind power and load forecasting methods for the optimal management of isolated power systems. En Proceedings of EWEC 1999 (European Wind Energy Conference). 1999.

Kariniotakis, G., Nogaret, E., Stavrakakis, G. et Al. Advanced short-term forecasting of wind power production. En European wind energy conference. Proceedings. EWEC'97, páginas 751-754. 1997.

Kaura, V. y Blasko, V. Operation of a phase locked loop system under distorted utility conditions. IEEE Trans. on Industry Applications, vol. 33(1), páginas 58-63, 1997. ISSN 0093-9994.

Kavasseri, R. y Seetharaman, K. Day-ahead wind speed forecasting using $<\mathrm{i}>\mathrm{f}</ \mathrm{i}>$-arima models. Renewable Energy, vol. 34(5), páginas 1388-1393, 2009.

Klinge Jacobsen, H. y Schröder, S. T. Curtailment of renewable generation: Economic optimality and incentives. Energy Policy, 2012.

Kristoffersen, J. The Horns Rev wind farm and the operational experience with the wind farm main controller. En Proc. Offshore Wind. Copenhagen (Denmark), 2005.

Kumar, P., Gupta, S. C. y Gupta, B. Frequency deviation transducer for power system applications. IEEE Trans. on Power Apparatus and Systems, vol. PAS-94, páginas 1270-1273, 1975.

Kusiak, A., Zheng, H. y Song, Z. Short-term prediction of wind farm power: a data mining approach. Energy Conversion, IEEE Transactions on, vol. 24(1), páginas 125-136, 2009.

Lang, S., Möhrlen, C., Jørgensen, J., Gallachóir, B. y McKeogh, E. Application of a multi-scheme ensemble prediction system for wind power forecasting in ireland and comparison with validation results from denmark and germany. system, vol. 2001, página 5, 2002.

Larsén, X., Vincent, C. y Larsen, S. Spectral structure of mesoscale winds over the water. Quarterly Journal of the Royal Meteorological Society, 2012.

LeOnhard, W. Control of Electrical Drives 3rd Edition. Springer, 2001.

Lipman, N., Bossanyi, E., Dunn, P., Musgrove, P., Whittle, G. y Maclean, C. Fluctuations in output from wind turbine clusters. Wind Engineering, vol. 4, páginas 1-7, 1980. 
Lobos, T. y Rezmer, J. Real-time determination of power system frequency. IEEE Trans. on Instrumentation and Measurement, vol. 46(4), páginas 877-881, 1997.

Makarov, Y., Hawkins, D., Leuze, E. y Vidov, J. California iso wind generation forecasting service design and experience. En Proc. of the 2002 AWEA Windpower Conference, Portland, Oregon. 2002.

Maqsood, I., Khan, M., Huang, G. y Abdalla, R. Application of soft computing models to hourly weather analysis in southern saskatchewan, canada. Engineering Applications of Artificial Intelligence, vol. 18(1), páginas 115-125, 2005.

Markou, H. y Larsen, T. J. Control strategies for operation of pitch regulated turbines above cut-out wind speeds. En European Wind Energy Conference and Exhibition (EWEC2009). 2009.

Martín-Martínez, S., Gómez-Lázaro, E., Molina-García, A., Fuentes, J. A., Vigueras-Rodríguez, A. y Amat Plata, S. A new three-phase dpll frequency estimator based on nonlinear weighted mean for power system disturbances. Power Delivery, IEEE Transactions on, vol. 28, páginas 179 - 187, 2013.

Martín-Martínez, S., Gómez-Lázaro, E. y Vigueras-Rodríguez, A. Comparison of power fluctuations from onshore and offshore wind farms. En IEA Task 23 Back-to-Back Workshop: Offshore wind farms - wake effects and power fluctuations. Roskilde, Denmark, 2009a.

Martín-Martínez, S., Gómez-Lázaro, E. y Vigueras-Rodríguez, A. Regulation technique for smoothing active power in aggregated wind farms distributed within spain. En 8th International Workshop on Large-Scale Integration of Wind Power. 2009b.

Martín-Martínez, S., Gómez-Lázaro, E., Vigueras-Rodríguez, A., Fuentes-Moreno, J. A. y Molina-García, A. Reducing wind power fluctuations in a system by applying ramp limitations to wind farms with greater oscillations. En Nordic Wind Power Conference. Bornholm, Denmark, 2009c. ISBN 978-87-92465-06-1.

Martín-Martínez, S., Gómez-Lázaro, E., Vigueras-Rodríguez, A., Fuentes-Moreno, J. A. y Molina-García, A. Regulation strategies for wind power fluctuations depending on demand (in spain). En 8th International Workshop on Large-Scale Integration of Wind Power. Quebec (Canada), 2010a.

Martín-Martínez, S., Gómez-Lázaro, E., Vigueras-Rodríguez, A., Fuentes-Moreno, J. A. y Molina-García, A. Regulation strategies 
for wind power fluctuations in a system depeding on ramping power range. En European Wind Energy Conference and Exhibition (EWEC2010). Warsaw (Poland), 2010b.

Martín-Martínez, S., Gómez-Lázaro, E., Vigueras-Rodríguez, A., Fuentes-Moreno, J. A. y Molina-García, A. Analysis of positive ramp limitation control strategies for reducing wind power fluctuations. IET Renewable Power Generation, vol. aceptado para publicación en revista, 2013 .

Martín-Martínez, S., Gómez-Lázaro, E., Vigueras-Rodríguez, A., Molina-García, A., Milligan, M. y Muljadi, E. Participation of wind power plants in the spanish power system during events. En IEEE PES General Meeting, San Diego, USA, página 8. 2012.

Martín-Martínez, S., Vigueras-Rodríguez, A., Gómez-Lázaro, E., Molina-García, A., Muljadi, E. y Milligan, M. Advances in wind power, capítulo Wind Power Variability and Singular Events, páginas 285304. InTech, 2012.

Milligan, M., Donohoo, P., Lew, D., Ela, E., Kirby, B., Holttinen, H., Lannoye, E., Flynn, D., O’Malley, M., Miller, N. Et Al. Operating reserves and wind power integration: an international comparison. En proc. 9th International Workshop on large-scale integration of wind power into power systems, páginas 18-29. 2010.

Milligan, M., Schwartz, M. y Wan, Y. Statistical wind power forecasting models: results for us wind farms. National Renewable Energy Laboratory, Golden, CO, 2003.

Minnesota. Minnesota wind integration study, volumes i and ii. Informe técnico, EnerNex Corp. and Windlogics Inc., 2006.

Monteiro, C., Keko, H., Bessa, R., Miranda, V., Botterud, A., Wang, J. y Conzelmann, G. A quick guide to wind power forecasting: State-of-the-art. Informe técnico, Argonne national laboratory, 2009.

Moore, P., Allmeling, J. y Johns, A. Frequency relaying based on instantaneous frequency measurement. IEEE Trans. on Power Delivery, vol. 11(4), páginas 1737-1742, 1996.

Nanahara, T., Asari, M., Sato, T., Yamaguchi, K., Shibata, M. y Maejima, T. Smoothing effects of distributed wind turbines. part 1. coherence and smoothing effects at a wind farm. WIND ENERGY, vol. 7, páginas 61-74, 2004a. 
Nanahara, T., Asari, M., Sato, T., Yamaguchi, K., Shibata, M. y MaEjima, T. Smoothing effects of distributed wind turbines. part 2. coherence among power output of distant wind turbines. WIND ENERGY, vol. 7, páginas 75-85, 2004b.

Nguyen, C. T. y Srinivasan, K. A new technique for rapid tracking of frequency deviations based on level crossings. IEEE Trans. on Power Apparatus and Systems, vol. PAS-103(8), páginas 2230-2236, 1984.

Nichita, C., Luca, D., Dakyo, B. y Ceanga, E. Large band simulation of the wind speed for real time wind turbine simulators. Energy Conversion, IEEE Transactions on, vol. 17(4), páginas 523-529, 2002.

Ontario. Ontario wind integration study. Informe técnico, GE Energy and AWS Truewind, 2006.

Parsons, B., Milligan, M., Zavadil, B., Brooks, D., Kirby, B., DraGOON, K. y CALDwell, J. Grid impacts of wind power: A summary of recent studies in the United States. Wind Energy, vol. 7(2), páginas 87$108,2004$.

Petru, T. y Thiringer, T. Modeling of wind turbines for power system studies. Power Systems, IEEE Transactions on, vol. 17(4), páginas 11321139, 2002.

Pinson, P., Christensen, L., Madsen, H., Sørensen, P., Donovan, M. y Jensen, L. Regime-switching modelling of the fluctuations of offshore wind generation. Journal of Wind Engineering and Industrial Aerodynamics, vol. 96(12), páginas 2327-2347, 2008.

Pinson, P. y Madsen, H. Adaptive modelling and forecasting of offshore wind power fluctuations with markov-switching autoregressive models. Journal of Forecasting, vol. 31(4), páginas 281-313, 2010.

Pinson, P., Madsen, H., Sørensen, P. y Cutululis, N. Adaptive modelling of offshore wind power fluctuations. En Proc. Nordic Wind Power Conference (NWPC) 2007, Nov. 200\%, Roskilde, Denmark. 2007.

Pinson, P., Siebert, N. y Kariniotakis, G. Forecasting of regional wind generation by a dynamic fuzzy-neural networks based upscaling approach. En Proceedings EWEC 2003. 2003.

Poggi, P., Muselli, M., Notton, G., Cristofari, C. y Louche, A. Forecasting and simulating wind speed in corsica by using an autoregressive model. Energy conversion and management, vol. 44(20), páginas 31773196, 2003. 
Potter, C., Grimit, E. y Nijssen, B. Potential benefits of a dedicated probabilistic rapid ramp event forecast tool. En Power Systems Conference and Exposition, 2009. PSCE'09. IEEE/PES, páginas 1-5. IEEE, 2009.

Potter, C. y Negnevitsky, M. Very short-term wind forecasting for tasmanian power generation. Power Systems, IEEE Transactions on, vol. 21(2), páginas 965-972, 2006.

Ramírez-Rosado, I. y Fernández-Jiménez, L. Next-day wind park electric energy generation forecasting using fuzzy time-series. relation, vol. 1000, página 6, 2003.

Ramirez-Rosado, I., Fernandez-Jimenez, L., Monteiro, C., Sousa, J. y BESSA, R. Comparison of two new short-term wind-power forecasting systems. Renewable Energy, vol. 34(7), páginas 1848-1854, 2009.

Rauh, A. y Peinke, J. A phenomenological model for the dynamic response of wind turbines to turbulent wind. Journal of wind engineering and industrial aerodynamics, vol. 92(2), páginas 159-183, 2004.

RAZABI, R. Monolithic phase-locked loop and clock recovery circuit. IEEE Press, 1996.

REE. Procedimiento de opercación 1.5.: . Establecimiento de la reserva para la regulación frecuencia-potencia", boe 13 de julio de 2006 edición, 2006.

REE. Procedimiento de opercación 3.7.: "Programación de la generación de origen renovable no gestionable", boe 28 de mayo de 2009 edición, 2009.

REE. Red electrica de españa. www.ree.es.

Ren, B., Zhong, Y., Sun, X. y Tong, X. A digital pll control method based on the fir filter for a grid-connected single-phase power conversion system. En IEEE International Conference on Industrial Technology.. 2008 .

Ren, J. y Kezunovic, M. Real-time power system frequency and phasors estimation using recursive wavelet transform. IEEE Trans. on Power Delivery,, (99), páginas 1392-1402, 2011.

Rogers, J., Fink, S. y Porter, K. Examples of wind energy curtailment practices. NREL, Subcontract Report NREL/SR-550-48737, 2010.

Rosas, P. Dynamic influences of wind power on the power system. Tesis Doctoral, Technical University of Denmark, 2003.

RWE. The need for smart megawatts power generation in europe, facts \& trends. Informe técnico, RWE, 2009. 
SACHDEV, M. S. Advancement in microprocessor-based protection and communication. IEEE Tutorial Course Text, 1997.

Sachdev, M. S. y Giray, M. M. A least error squares technique for determining power system frequency. IEEE Trans. on Power Apparatus and Systems, vol. PAS-104(2), páginas 437-443, 1985.

SAChdev, M. S. y Nagpal, M. A recursive least error squares algorithm for power system relaying and measurement applications. IEEE Trans. Power Delivery, vol. 6, páginas 1008-1015, 1991.

Saitou, N., Matsui, M. y Shimizu, T. A control strategy of single-phase active filter using a novel d-q transformation. En Proc. 38th Industry Applications Society Annual Meeting. Salt Lake City, USA, 2003.

Sakamoto, S., Izumi, T., Yokoyama, T. y Haneyoshi, T. A new method for digital pll control using estimated quadrature two phase frequency detection. En Proc. Power Conversion Conference. Osaka, 2002.

Salcic, Z., Nguang, S. y Wu, Y. An improved taylor method for frequency measurement in power systems. IEEE Trans. on Instrumentation and Measurement,, vol. 58(9), páginas 3288-3294, 2009.

Saranyasoontorn, K., Manuel, L. y Veers, P. A comparison of standard coherence models for inflow turbulence with estimates from field measurements. Transactions of the ASME-N-Journal of Solar Energy Engineering, vol. 126(4), páginas 1069-1082, 2004.

Schlez, W. y INFIELD, D. Horizontal, two point coherence for separations greater than the measurement height. Boundary-Layer Meteorology, vol. 87(3), páginas 459-480, 1998.

Schlink, U. y Tetzlaff, G. Wind speed forecasting from 1 to 30 minutes. Theoretical and applied climatology, vol. 60(1), páginas 191-198, 1998.

Sfetsos, A. A comparison of various forecasting techniques applied to mean hourly wind speed time series. Renewable Energy, vol. 21(1), páginas 2335,2000 .

Sfetsos, A. A novel approach for the forecasting of mean hourly wind speed time series. Renewable Energy, vol. 27(2), páginas 163-174, 2002.

SieBert, N. Development of methods for regional wind power forecasting. Tesis Doctoral, Ecole des mines de Paris, 2008.

Siebert, N., Kariniotakis, G. et al. Reference wind farm selection for regional wind power prediction models. En Proceedings of the European Wind energy conference, EWEC 2006. 2006. 
Silva, S., Lopes, B., Filho, B., Campana, R. y Bosventura, W. Performance evaluation of pll algorithms for single-phase grid-connected systems. En Proc. 39th IEEE Industry Applications Annual Meeting Conference, 2004.

da Silva, S., Tomizaki, E., Novochadlo, R. y Coelho, E. Pll structures for utility connected systems under distorted utility conditions. En Proc. 32nd Annual Conference on IEEE Industrial Electronics. 2006.

Sinden, G. Characteristics of the uk wind resource: Long-term patterns and relationship to electricity demand. Energy Policy, vol. 35(1), páginas 112-127, 2007.

SlootweG, J. Wind power: Modelling and impact on power system dynamics. Tesis Doctoral, 2003.

Slootweg, J., De Haan, S., Polinder, H. y Kling, W. General model for representing variable speed wind turbines in power system dynamics simulations. Power Systems, IEEE Transactions on, vol. 18(1), páginas 144-151, 2003.

Smith, J., Milligan, M., DeMeo, E. y Parsons, B. Utility wind integration and operating impact state of the art. Power Systems, IEEE Transactions on, vol. 22(3), páginas 900-908, 2007.

Soder, L., Hofmann, L., Orths, A., Holttinen, H. Wan, Y. y Tuohy, A. Experience from wind integration in some high penetration areas. IEEE Transactions on Energy Conversion,, vol. 22 Issue: 1, páginas 4-12, 2007.

Solari, G. Turbulence modeling for gust loading. Journal of Structural Engineering, vol. 113, página 1550, 1987.

Song, H.-S., NAm, K. y Mutschler, P. Very fast phase angle estimation algorithm for a single-phase system having sudden phase angle jumps. En Proc. 37th IEEE Industry Applications Annual Meeting Conference,. 2002.

Sørensen, P., Cutululis, N., Vigueras-Rodríguez, A., Madsen, H., Pinson, P., Jensen, L., Huerrild, J. y Donovan, M. Modelling of power fluctuations from large offshore wind farms. Wind Energy, vol. 11(1), páginas 29-43, 2008.

Sørensen, P., Hansen, A. y Rosas, P. Wind models for simulation of power fluctuations from wind farms. Journal of Wind Engineering and Industrial Aerodynamics, vol. 90(12), páginas 1381-1402, 2002.

Sørensen, P., Mann, J., Paulsen, U. y Vesth, A. Wind farm power fluctuations. Wind Energy, páginas 139-145, 2007. 
Soto, F. y Prieto, E. Spanish experience in the integration of wind and solar energy into the electric power system. En Integration of Wide-Scale Renewable Resources Into the Power Delivery System, 2009 CIGRE/IEEE PES Joint Symposium. 2009.

Spahic, E. y Balzer, G. Power fluctuation from a large wind farm. En 2005 International Conference on Future Power Systems. 2005.

Sumannarat, A., Bak-Jensen, B., Chen, Z., Nielsen, H., Huerrild, J., Sorensen, P. y Hansen, A. D. Power system operation with large scale wind power integration. En Power Tech, 2007 IEEE Lausanne, páginas 671-676. IEEE, 2007.

Szafran, J. y Rebizant, W. Power system frequency estimation. IEEE Proc. on Generation, Transmission and Distribution, vol. 145(5), páginas 221-226, 1998.

Tamuraa, Y., Kareemb, A., Solaric, G., Kwokd, K. y Holmese, J. Report by working group wge-dynamic response. En Pacific Conference on Wind Engineering held in Kyoto from October, vol. 22, página 24. 2001.

TeRzIJA, V. Improved recursive newton-type algorithm for frequency and spectra estimation in power systems. IEEE Trans. on Instrumentation and Measurement, vol. 52(5), páginas 1654-1659, 2003.

Vanmarcke, E. Random fields: analysis and synthesis. World Scientific Publishing Company Incorporated, 2010.

Veers, P. Three-dimensional wind simulation. Informe técnico, Sandia National Labs., Albuquerque, NM (USA), 1988.

Vigueras-Rodríguez, A. Modelling of the Power Fluctuations in Large Offshore Wind Farms. Tesis Doctoral, Universidad Politécnica de Cartagena, 2008.

Vigueras-Rodríguez, A., Sørensen, P., Cutululis, N., Viedma, A., Gomez, E. y MARTin, S. Application of ramp limitation regulations for smoothing the power fluctuations from offshore wind farms. En European Wind Energy Conference and Exhibition (EWEC09). Marseille (France), 2009.

Vincent, C., Larsén, X., Larsen, S. y Sørensen, P. Cross-spectra over the sea from observations and mesoscale modelling. Boundary-Layer Meteorology, páginas 1-22, 2012.

Von Karman, T. Progress in the statistical theory of turbulence. Proceedings of the National Academy of Sciences of the United States of America, vol. 34(11), página 530, 1948. 
WAN, Y. A primer on wind power for utility applications. National Renewable Energy Laboratory, 2005.

Welch, P. The use of fast fourier transform for the estimation of power spectra: a method based on time averaging over short, modified periodograms. Audio and Electroacoustics, IEEE Transactions on, vol. 15(2), páginas 70-73, 1967.

Woods, M., Davy, R., Russell, C. y Coppin, P. Cross-spectrum of wind speed for meso-gamma scales in the upper surface layer over south-eastern australia. Boundary-layer meteorology, vol. 141(1), páginas 93-116, 2011.

Yahaya, S., Frangi, J., Richard, D. ET al. Turbulent characteristics of a semiarid atmospheric surface layer from cup anemometers-effects of soil tillage treatment (northern spain). En Annales Geophysicae, vol. 21, páginas 2119-2131. 2003.

YANG, J. y LiU, C. A precise calculation of power system frequency and phasor. IEEE Trans. on Power Delivery,, vol. 16(3), páginas 361-366, 2001.

YI, T. y Rahman, S. A framework of agent-based demand response to provide reserves necessary for high-level wind penetration. En Power and Energy Engineering Conference (APPEEC), 2010 Asia-Pacific, páginas 1-4. IEEE, 2010.

ZACK, J. W. Optimization of wind power production forecast performance during critical periods for grid management. En Proceedings of the European Wind Energy Conference EWEC, Milano (IT), vol. 8. 2007.

Zhang, Y. y Chowdhury, A. A. Reliability assessment of wind integration in operating and planning of generation systems. En Proc. IEEE Power. Energy Society General Meeting PES '09, páginas 1-7. 2009.

ZHU, Z. y YANG, H. Discrete hilbert transformation and its application to estimate the wind speed in hong kong. Journal of Wind Engineering and Industrial Aerodynamics, vol. 90(1), páginas 9-18, 2002. 

